\title{
A Phase 1 Dose Escalation Trial of Third Generation Chimeric Antigen Receptor (CAR) T- Cell Therapy for Relapsed and Refractory B-Cell Non Hodgkin Lymphoma
}

A thesis by publications presented in fulfilment of the requirements for the degree of:

\section{Masters of Clinical Research by Thesis Victoria University of Wellington}

Dr Philip George

August 2020 


\section{Abstract}

Anti-CD19 Chimeric Antigen Receptor (CAR) T-cell therapy is shifting the treatment paradigm internationally for selected patients with relapsed and refractory B-cell NonHodgkin Lymphoma. Despite high response rates with durable responses achieved in a significant proportion of patients, over $50 \%$ of patients will have progressed at one year following treatment with the currently licensed anti-CD19 CAR T-cell therapies. This modality of therapy is also associated with acute and potentially life-threatening toxicities, requiring strict risk mitigation strategies.

In this thesis, the design, preparation and implementation of a new third generation antiCD19 CAR T-cell Phase 1 trial entitled ENABLE, for patients with relapsed and refractory B-cell Non-Hodgkin Lymphoma, is described in detail. Following a literature review of CAR T-cell therapy in patients with B-cell Non-Hodgkin Lymphoma, the rationale for the ENABLE trial design is discussed, along with regulatory and clinical requirements for setting up CAR T-cell therapy in New Zealand. The importance of international collaboration to inform aspects of study design, CAR T-cell product manufacturing and developing CAR Tcell toxicity management protocols, has been demonstrated.

The early clinical experience on the ENABLE trial is presented along with provisional safety, pharmacokinetic and efficacy data from the first participant treated. This is the first time that CAR T-cell therapy has been administered in New Zealand, demonstrating CAR T-cell expansion in vivo; but also highlighting the complexities of the CAR T-cell product manufacturing process and the importance of evaluating feasibility of CAR T-cell manufacturing, as a key secondary endpoint of the study. Further clinical experience on the ENABLE trial is crucial to develop the potential for CAR T-Cell therapy to be a safe, feasible and effective option for selected New Zealand patients in the future. 


\section{Authors declaration}

This thesis was produced using contributions from peer reviewed journal publications for which I am first author or a co-author on. It also includes one chapter that is a manuscript in the final preparation for submission to a peer reviewed journal, for which I am the first author on (Chapter 4). Two of the published peer reviewed publications form stand-alone chapters (Chapter 3 and Chapter 5) in this thesis and my contribution to these manuscripts is clearly outlined in Chapter 1. Introduction. For Chapter 5, I was the first author and primary writer of the manuscript, making significant contributions to all sections of the manuscript. The third peer reviewed publication has been incorporated into the last sub section of Chapter 2. Literature review. I have only included extracts from this publication for which I have made significant contributions to and that fits into the context of the thesis. Along with my supervisor Dr Robert Weinkove, I also wrote the ENABLE Study protocol and Investigator Brochure and I took the lead in the following trial regulatory body submissions for the ENABLE trial: Health and Disability Ethics Committee, Gene Technology Advisory Committee, Research Advisory Group Māori and the Environmental Protection Agency.

\section{Acknowledgements}

There are a very large number of people who have helped me with the work required for this thesis. In particular I would like to thank:

My supervisor, Dr Robert Weinkove for his expert guidance, mentorship and enthusiasm during the process of setting up the ENABLE trial and during the writing of this thesis. I have learnt a huge amount about the process of clinical research from him, and thanks to his guidance, my research writing skills have significantly developed over the past two years. Rob has always encouraged me to think smartly about how best to gain output from an assignment or project, enabling me to embrace and benefit from a host of learning opportunities during this Masters period.

My colleague and friend at the Malaghan Institute, Nathaniel Dasyam, who has worked closely with me on a number of review articles which have contributed towards this thesis. 
Thank you for explaining and teaching me about the laboratory side of CAR T-cell therapy research, educating me on how to use Prism, and for other moral support during this project!

My colleague and friend Tess Ostapowicz, the Study Nurse for the ENABLE trial, who has worked very closely with me on the clinical aspects of running the ENABLE trial. Thank you for your positive approach and encouragement during this project!

The GMP team at the Malaghan Institute including Giulia Giunti, Brigitta Mester, Bethany Andrews, Evelyn Bauer, Regina Duffield and Ian Hermans; whose hard work and dedication have been of paramount importance in setting up and running the ENABLE trial. I've learnt a lot about the process of cellular therapy and developing the infrastructure of a clinical trial from working with them, and it has been a fun and enjoyable process along the way!

My colleagues at Capital and Coast District Health Board including Travis Perera, Alwyn D'Souza and Catherine Tang. I have enjoyed working closely with them all on the ENABLE trial TMC and have benefited from their expertise and from many interesting clinical discussions with them.

Professor Graham LeGros and the rest of the staff at the Malaghan Institute for their support during my time at the Malaghan and for making the Institute such a great place to work!

The Florence Peterson Leukaemia Trust for funding my clinical fellowship and enabling me to embrace this fantastic opportunity in clinical research!

My parents Robin and Celine and my sister Sophie, for their advice, encouragement and support from across the world!

Lastly, and most importantly to my wife Niru, for her love, support, encouragement and honesty during this time. I love you very much. 


\section{Table of Contents}

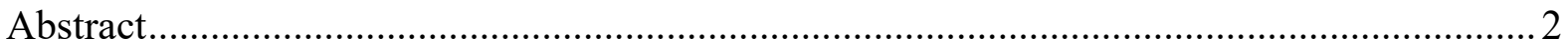

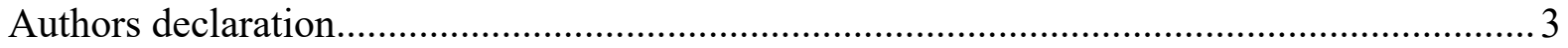

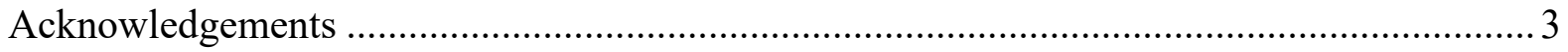

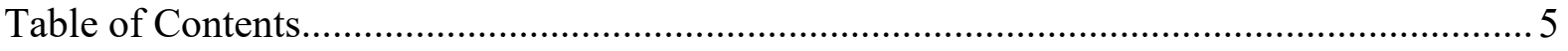

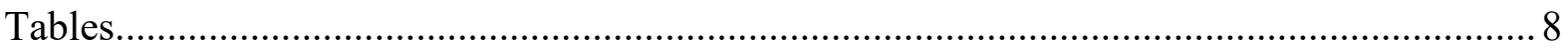

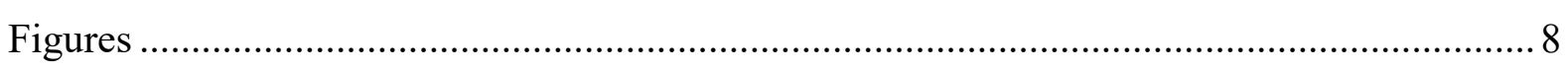

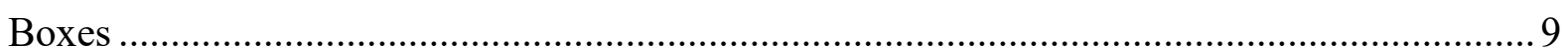

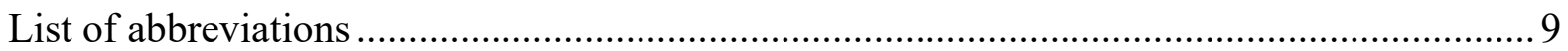

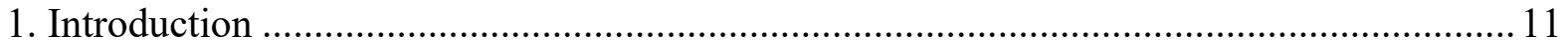

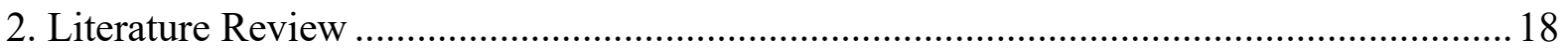

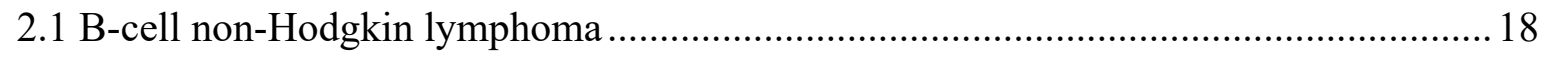

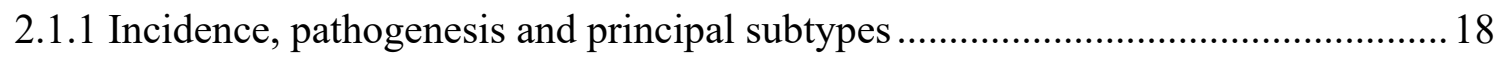

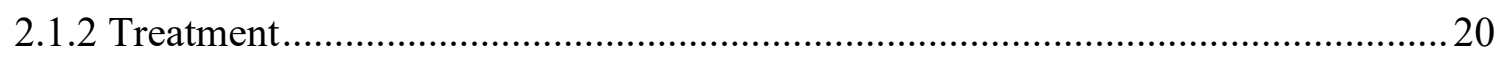

2.1.3 Management of relapsed and refractory disease .............................................2 21

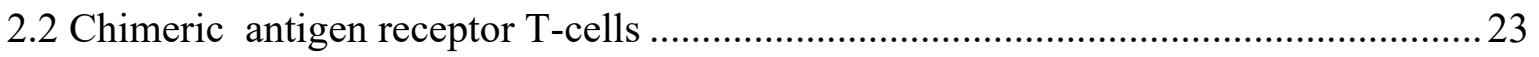

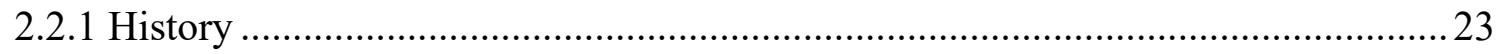

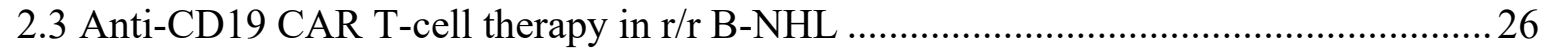

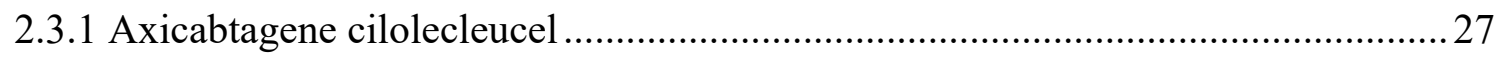

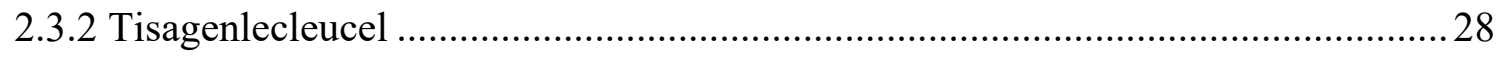

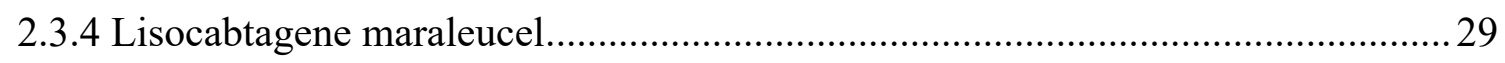

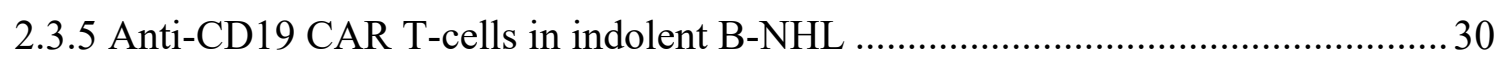

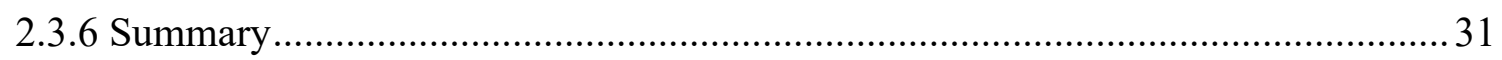

2.4 Limitations in efficacy of current anti-CD19 CAR T-cells in r/r B-NHL ......................34

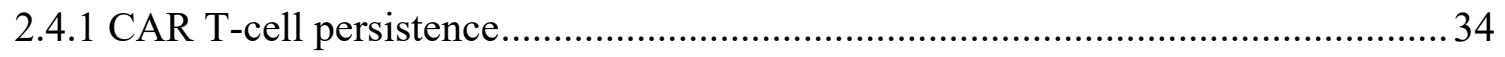

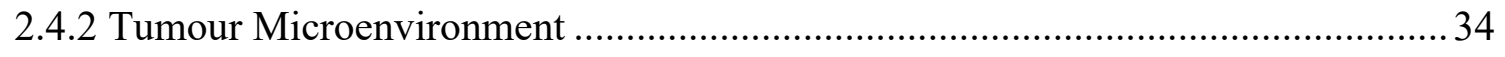

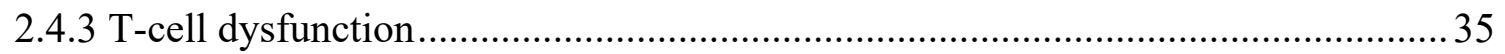

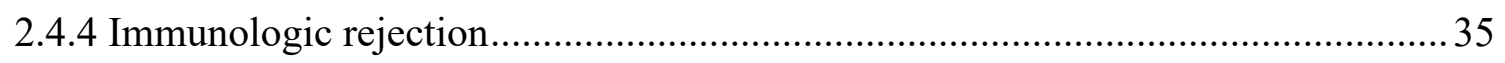

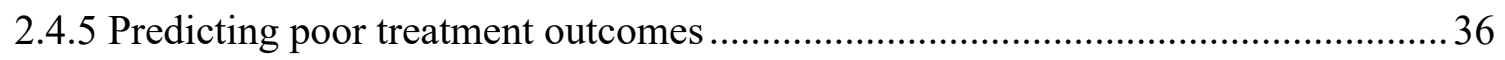

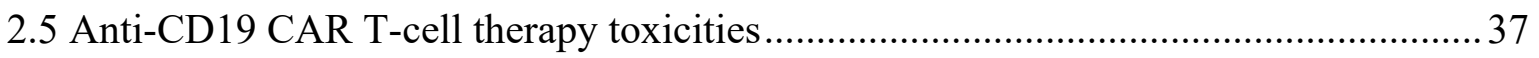

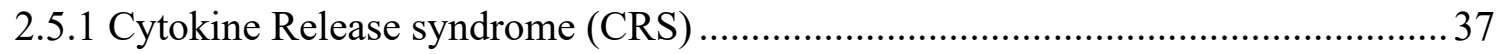

2.5.2 Immune Effector Cell-associated Neurotoxicity syndrome (ICANS)................... 41

2.5.3 Other anti-CD19 CAR T-cell toxicities............................................................ 47

2.6 Selecting costimulatory domains for chimeric antigen receptors: functional and clinical

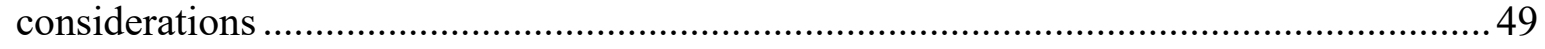

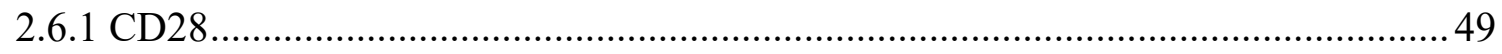

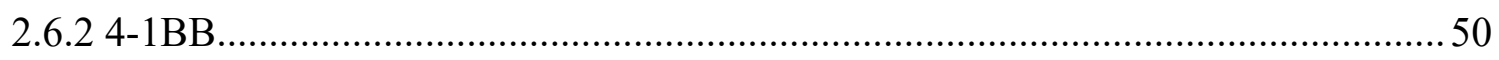


2.6.3 Toll like receptors

2.6.4 Third generation CARs - combining costimulatory domains...............................51

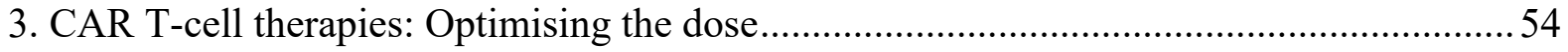

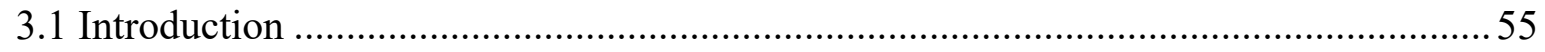

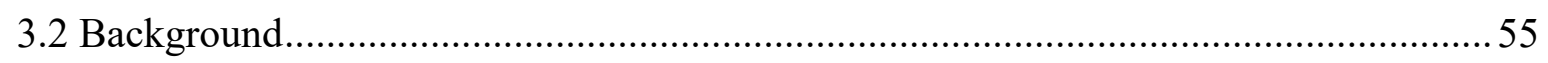

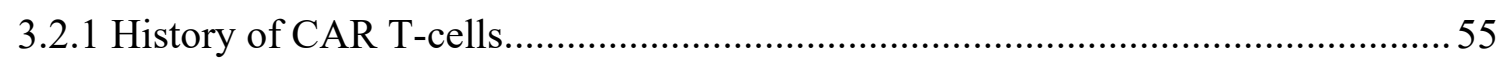

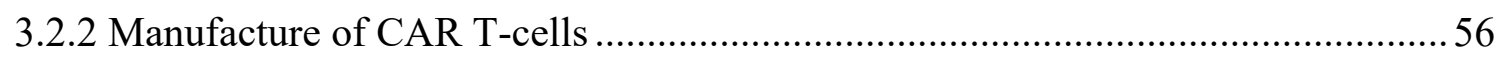

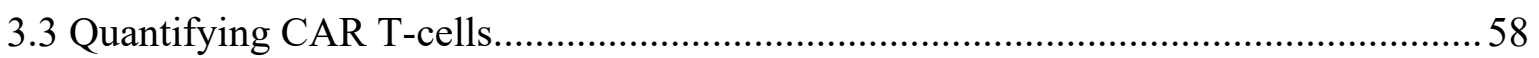

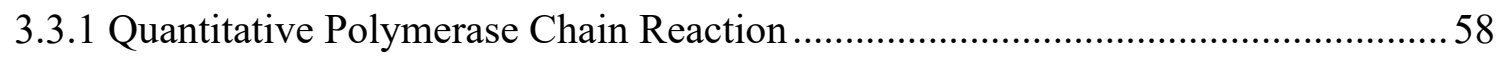

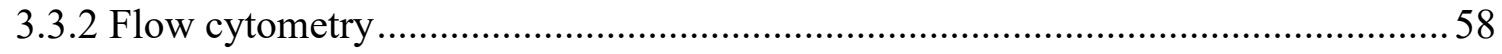

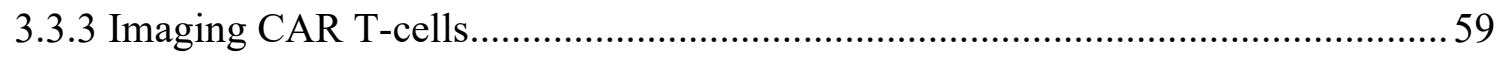

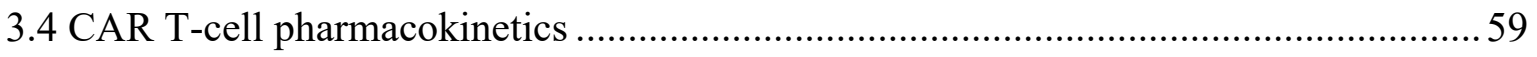

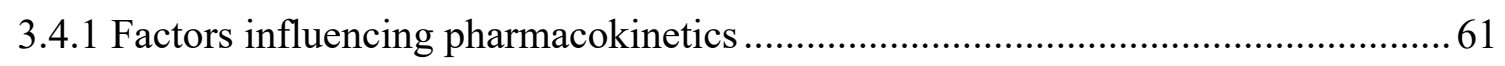

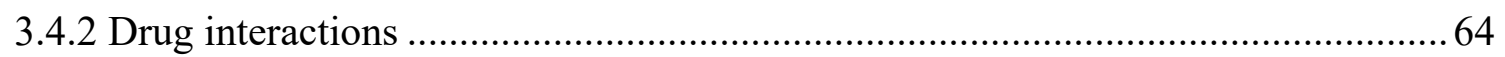

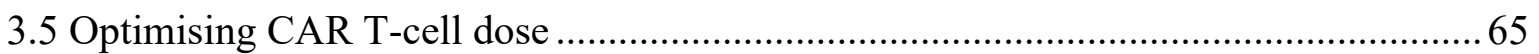

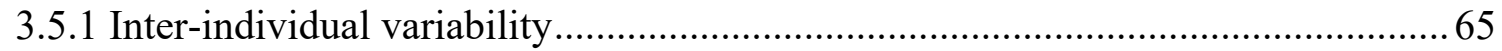

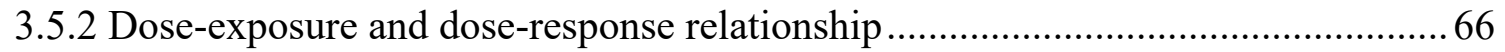

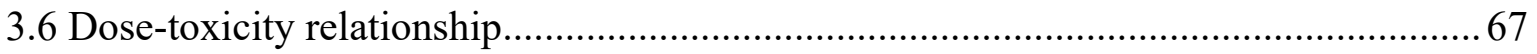

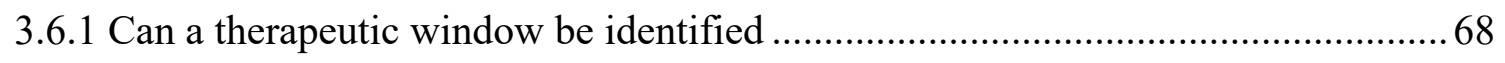

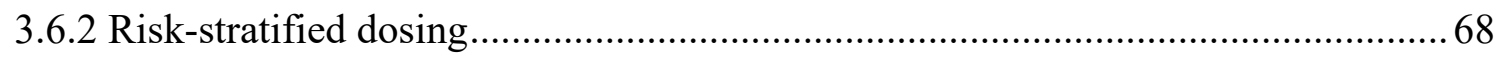

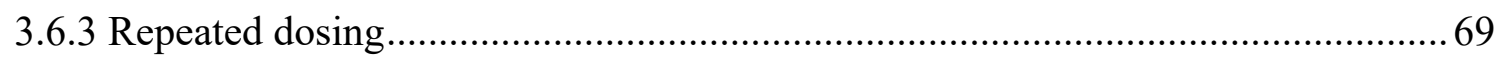

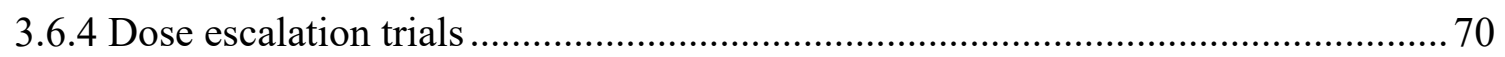

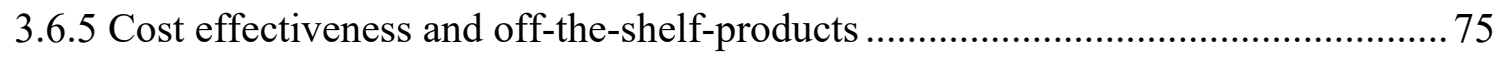

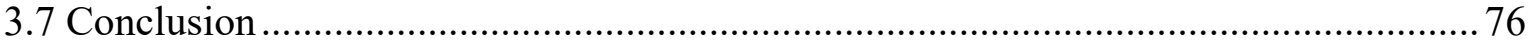

4. Chimeric antigen receptor T-cells in New Zealand: Challenges and Opportunities ............77

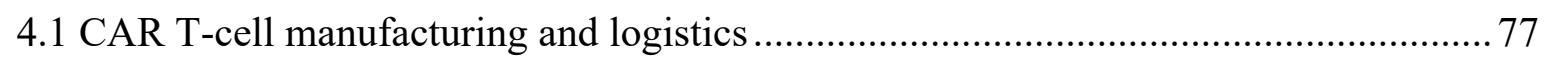

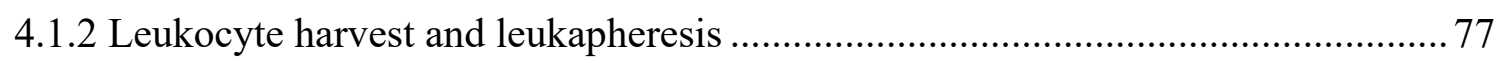

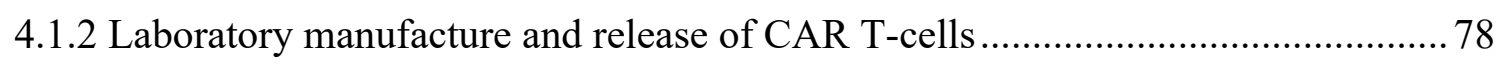

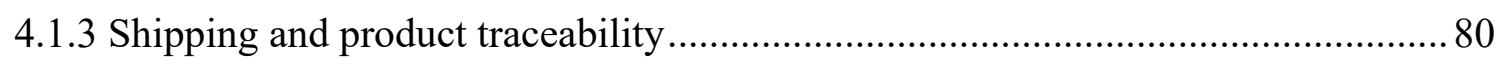

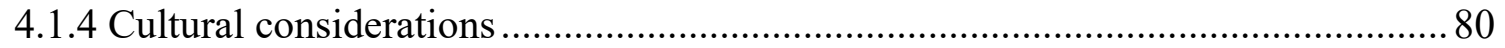

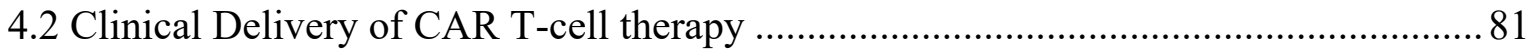

4.2.1 CAR T-cell toxicity management working group ............................................. 81

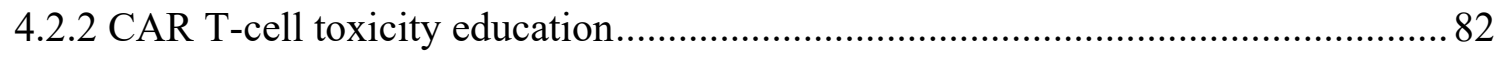

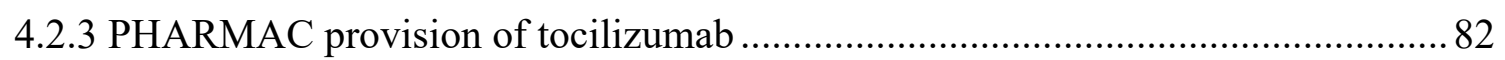

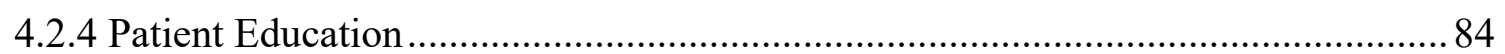

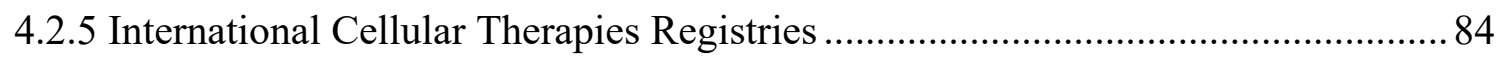

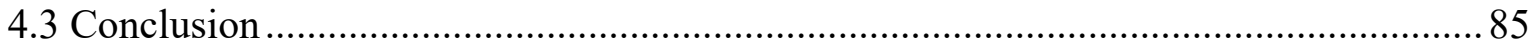


5. Third-generation anti-CD19 chimeric antigen receptor T-cells incorporating a TLR2 domain for relapsed or refractory B-cell lymphoma: a phase I clinical trial protocol

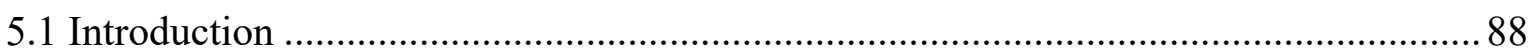

5.1.1 CAR T-cell therapy for B-cell non-Hodgkin lymphoma...................................... 88

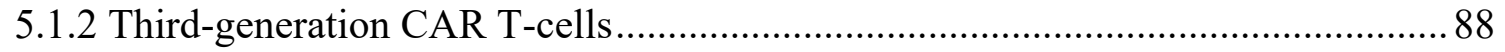

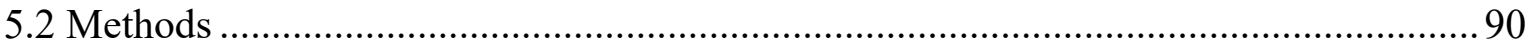

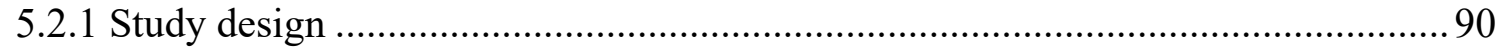

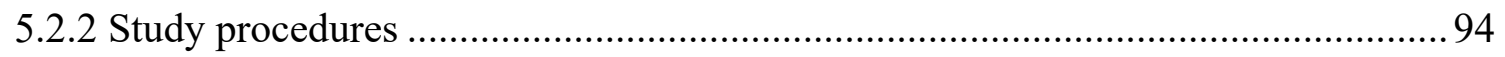

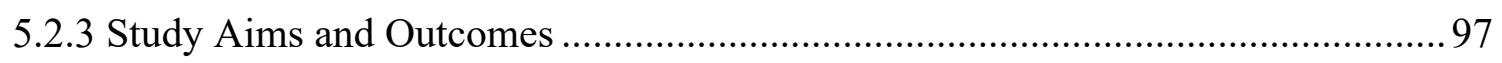

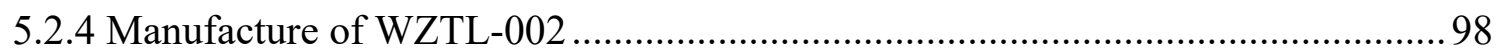

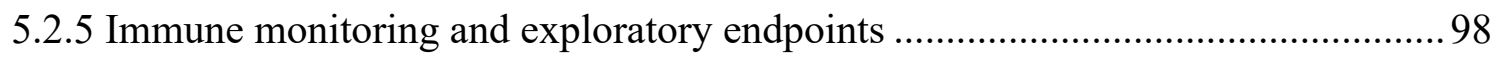

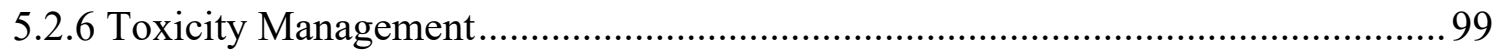

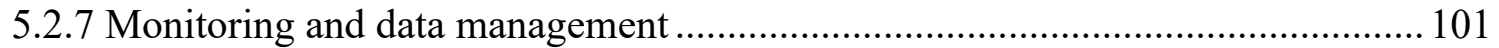

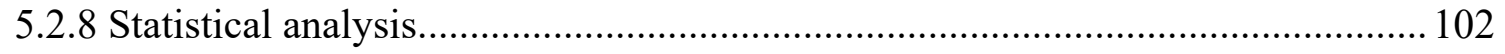

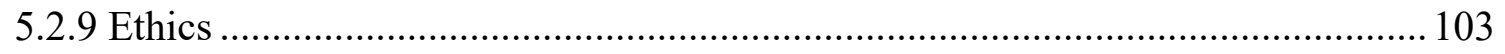

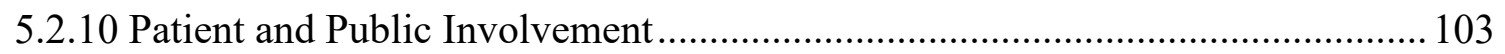

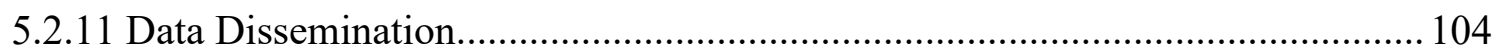

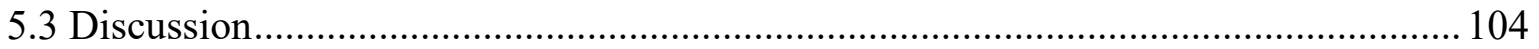

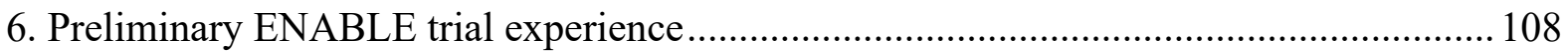

6.1 Referrals, pre-screening and screening experience ................................................ 108

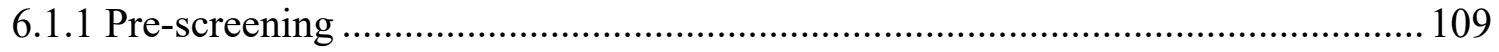

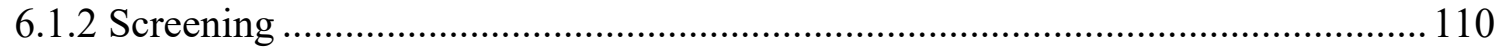

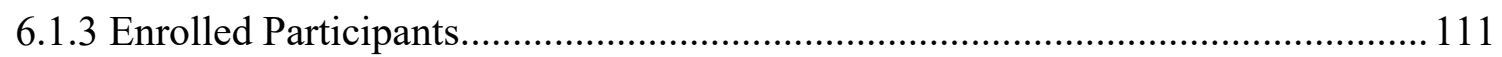

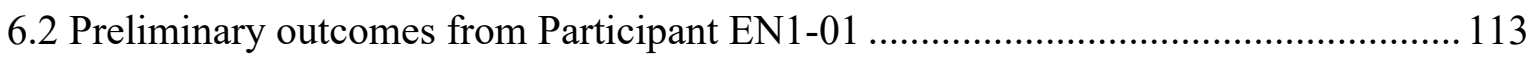

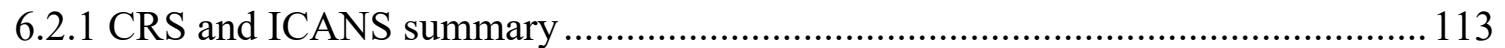

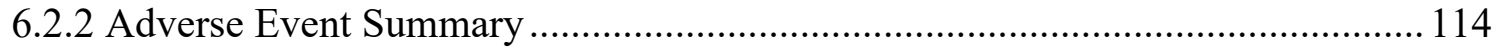

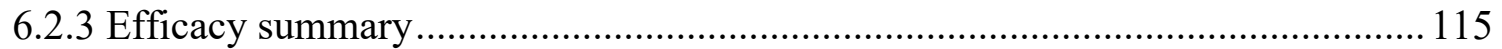

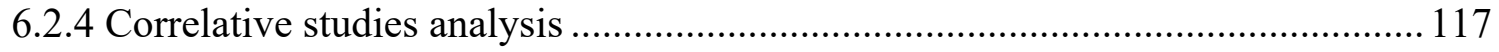

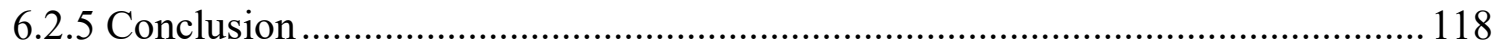

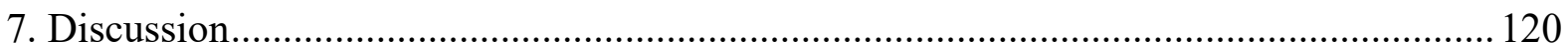

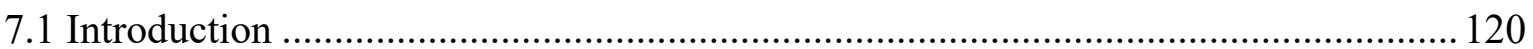

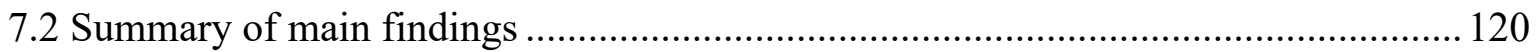

7.3 Engagement with New Zealand regulatory bodies.................................................. 122

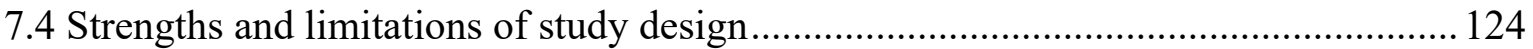

7.5 Protocol modifications based on early trial experience ............................................. 126

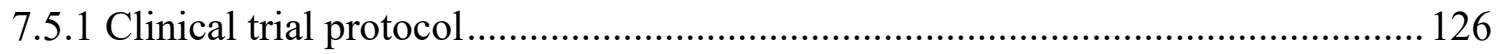




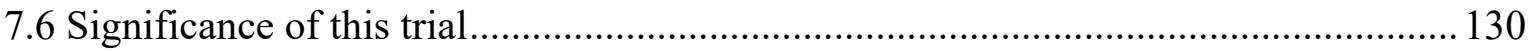

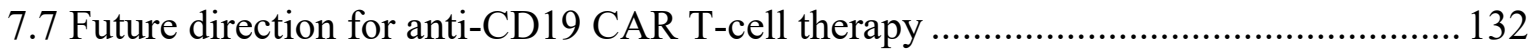

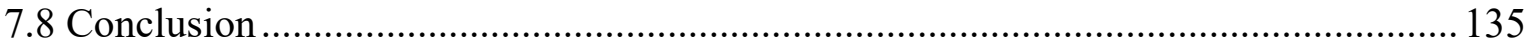

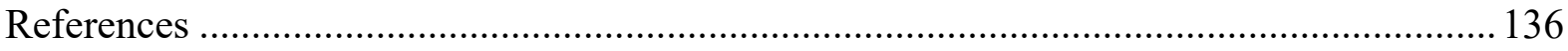

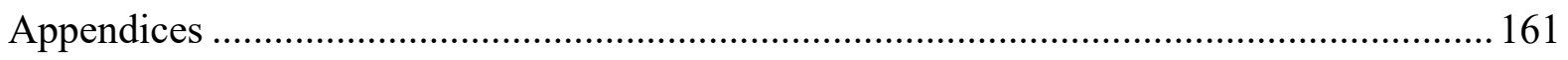

Appendix 1: Quicksheet for CRS management algorithm adopted for the ENABLE Trial in

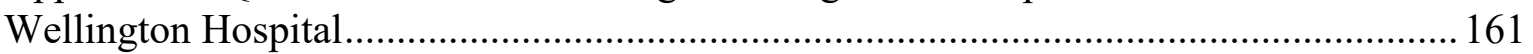

Appendix 2: Quicksheet for ICANS management algorithm adopted for the ENABLE Trial

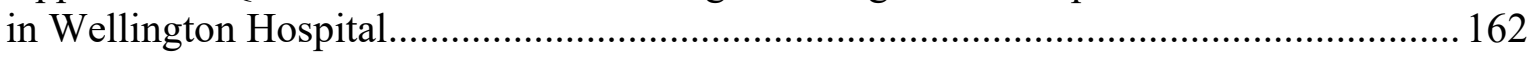

Appendix 3 Additional Manuscripts contributed to during Masters thesis ....................... 162

\section{Tables}

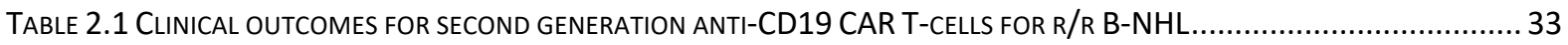

TABLE 2.2 CRS AND NEUROTOXICITY INCIDENCE IN TRIALS OF ANTI-CD19 CAR T-CELLS FOR R/R B-NHL ...............................46

TABLE 4.1 REgULATORY REQUIREMENTS FOR MANUFACTURING AND DELIVERING CAR T-CELL THERAPY WITHIN NEW ZEALAND ..... 83

TABLE 5.1 OTHER THIRD GENERATION ANTI-CD19 CAR T-CELL TRIALS REGISTERED ON CLINICALTRIALS.GOV ............................107

TABLE 6.1 REASONS FOR PRE-SCREENING INELIGIBILITY ON THE ENABLE TRIAL UNTIL APRIL 2020.................................... 109

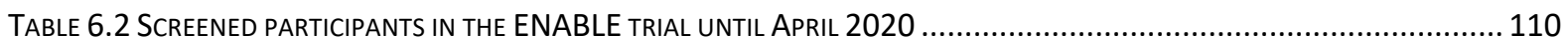

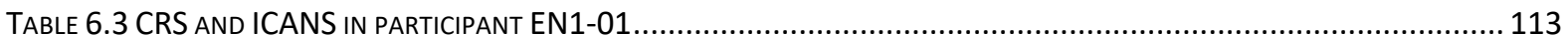

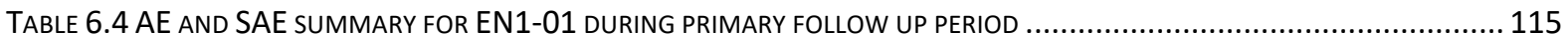

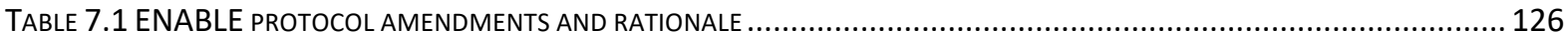

TABLE 7.2 SUMMARY OF KEY MANUFACTURING CHANGES TAKEN TO OPTIMISE WZTL-002 MANUFACTURING ......................... 129

\section{Figures}

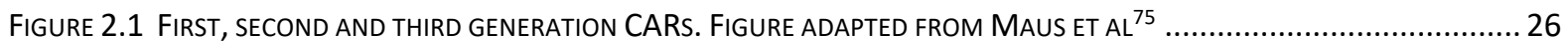

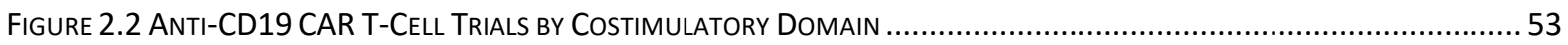

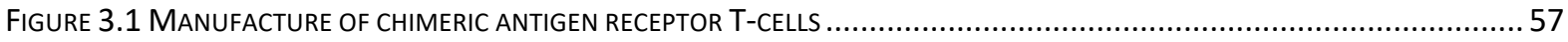

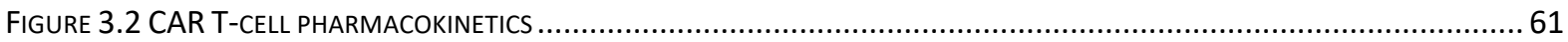

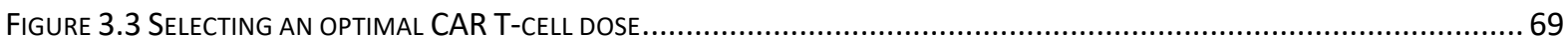

FIGURE 3.4 CONSORT DIAGRAM FOR ANTI-CD19 CAR T-CELL TRIAL DOSING INFORMATION OBTAINED FROM CLINICAL TRIALS.GOV

ERROR! BOOKMARK NOT DEFINED.

FIGURE 3.5 DOSES FROM ANTI-CD19 CAR T-CELL TRIALS REGISTERED ON CLINICALTRIALS.GOV .......................................... 73

FIGURE 4.1 VISUAL SUMMARY OF CAR T-CELL MANUFACTURING PROCESS ….......................................................... 79

FIGURE 5.1 DIAGRAMMATIC REPRESENTATION OF WZTL-002 ANTI-CD19 THIRD GENERATION CAR T-CELL ILLUSTRATING THE CO-

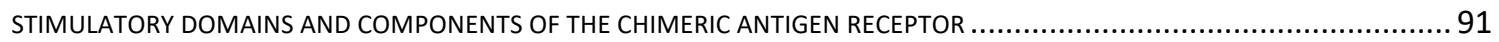

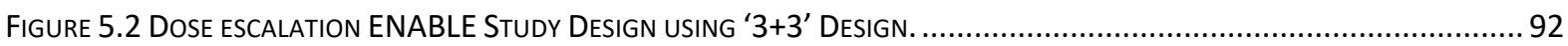

FIGURE 5.3 SCHEMA FOR THE ENABLE PHASE I DOSE ESCALATION STUDY. SECOND ATTEMPT AT CELL HARVEST AND WZTL-002 PRODUCTION MAY BE CONSIDERED AT DISCRETION OF TMC. SIX-MONTH PET SCAN IF FIRST PET SCAN POST WZTL-002 TREATMENT SHOWS PARTIAL RESPONSE..... 


\section{Boxes}

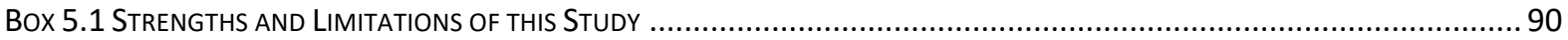

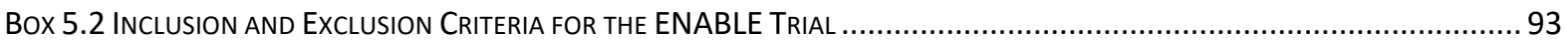

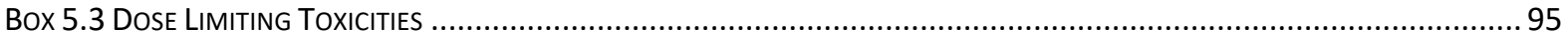

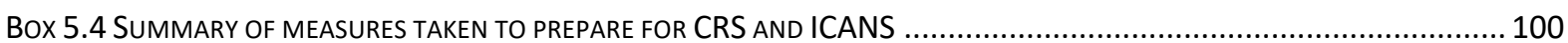

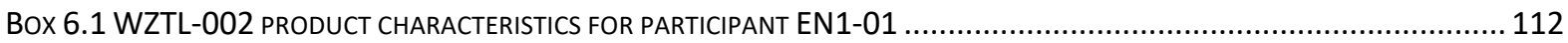

\section{List of abbreviations}

\begin{tabular}{|l|l|}
\hline Allo-SCT & Allogeneic stem cell transplant \\
\hline ASTCT & American Society for Transplantation and Cellular Therapy \\
\hline B-ALL & B-cell Acute Lymphoblastic Leukaemia \\
\hline BM & Bone Marrow \\
\hline BMAT & Bone Marrow Aspirate \& Trephine \\
\hline CAR & Chimeric Antigen Receptor \\
\hline CD3 & Cluster of Differentiation 3 \\
\hline CD19 & Cluster of Differentiation 19 \\
\hline CD20 & Cluster of Differentiation 20 \\
\hline CD28 & Cluster of Differentiation 28 \\
\hline CIBMTR & Center for International Blood and Marrow Transplant Research \\
\hline CLL & Chronic Lymphocytic Leukaemia \\
\hline CNS & Central Nervous System \\
\hline CR & Complete response \\
\hline CRP & C-reactive protein \\
\hline CRS & Cytokine Release Syndrome \\
\hline CTCAE & Common terminology criteria for adverse events \\
\hline DLT & Dose Limiting Toxicity \\
\hline DLBCL & Diffuse large B-cell lymphoma \\
\hline DSMC & Data Safety Monitoring Committee \\
\hline ECOG & European Cooperative Oncology Group \\
\hline EFS & Event Free Survival \\
\hline EPA & Environmental Protection Agency \\
\hline FL & Follicular Lymphoma \\
\hline GCP & Good clinical practice \\
\hline GCSF & Granulocyte colony stimulating factor \\
\hline GFR & Glomerular filtration rate \\
\hline &
\end{tabular}




\begin{tabular}{|l|l|}
\hline GMO & Genetically Modified Organism \\
\hline GTAC & Gene Technology Advisory Committee \\
\hline GVHD & Graft versus host disease \\
\hline HBV & Hepatitis B virus \\
\hline HCV & Hepatitis C virus \\
\hline HDEC & Health and Disability Ethics Committee \\
\hline HIV & Human Immunodeficiency Virus \\
\hline HLA & Human Leukocyte Antigen \\
\hline HLH & Haemophagocytic Lymphohistiocytosis \\
\hline HSNO & Hazardous Substances and New Organisms \\
\hline ICANS & Immune Effector Cell-Associated Neurotoxicity Syndrome \\
\hline ICE & Immune Effector Cell Encephalopathy Score \\
\hline ICH GCP & $\begin{array}{l}\text { International Conference on Harmonisation, Good Clinical } \\
\text { Practice }\end{array}$ \\
\hline IFN $\gamma$ & Interferon gamma \\
\hline IL-6 & Interleukin-6 \\
\hline IMP & Investigation Medicinal Product \\
\hline LDH & Lactate Dehydrogenase \\
\hline MCL & Mantle cell lymphoma \\
\hline MED & Minimum Effective Dose \\
\hline MIMR & Malaghan Institute of Medical Research \\
\hline MUGA Scan & Multigated Acquisition Scan \\
\hline MTD & Maximum Tolerated Dose \\
\hline MZL & Marginal Zone Lymphoma \\
\hline NCI & National Cancer Institute \\
\hline NHL & Non-Hodgkin Lymphoma \\
\hline NZBS & New Zealand Blood Service \\
\hline OS & Overall Survival \\
\hline PBMCs & Peripheral Blood Mononuclear Cells \\
\hline PD & Progressive Disease \\
\hline PET/CT & $\begin{array}{l}\text { Positron-Emission Tomography/ Computed Tomography } \\
\text { (PET/CT) }\end{array}$ \\
\hline PFS & Progression Free Survival \\
\hline PI & Principal Investigator \\
\hline PICF & Patient Information \& Consent Form \\
\hline PMBCL & Primary mediastinal B-cell lymphoma \\
\hline $\mathrm{r} / \mathrm{r}$ & Relapsed and/or Refractory \\
\hline R-CHOP & $\begin{array}{l}\text { Rituximab, Cyclophsphamide, Doxyrubicin, Vincristine, } \\
\text { Prednisolone }\end{array}$ \\
\hline SAE & Serious Adverse Event \\
\hline SCC & Squamous Cell Carcinoma \\
\hline SCT & Stem Cell Transplant \\
\hline SD & \\
\hline SOP & \\
\hline
\end{tabular}




\begin{tabular}{|l|l|}
\hline TNF $\alpha$ & TNF alpha \\
\hline TLR2 & Toll Like Receptor 2 \\
\hline TMC & Trial Management Committee \\
\hline WBCC & Wellington Blood and Cancer Centre \\
\hline WHO & World Health Organisation \\
\hline WZTL-002 & $\begin{array}{l}\text { 1928T2z CAR T-cells generated at the Malaghan Institute of } \\
\text { Medical Research }\end{array}$ \\
\hline
\end{tabular}

\section{Introduction}

Chimeric Antigen Receptor (CAR) T-Cells are genetically modified T-cells, which express fusion proteins that include an antigen recognition domain and a T-cell signalling domain ${ }^{1}$. The receptors are chimeric because they combine antigen recognition and binding, with T-cell activating functions into a single receptor ${ }^{2}$. In CARs, the antigen recognition portion of the receptor is derived from an antibody (the B-cell receptor) and the T-cell signalling and activating portion of the receptor is derived from the T-cell receptor (TCR) ${ }^{3}$. Therefore, in contrast to endogenous T-cells, antigen recognition and activation of a CAR T-cell occurs in a Human Leukocyte Antigen (HLA) independent manner ${ }^{4}$. Expression of the CAR on the T-cell surface, is achieved by incorporating the gene encoding the CAR construct into the genomes of the T-cells using a vector (usually a replication incompetent $\gamma$-retrovirus or lentivirus) ${ }^{5}$. The first generation of CAR T-cells comprising the single chain variable fragment ( $\mathrm{scFv}$ ) of a monoclonal antibody as the antigen recognition domain, linked via a hinge and transmembrane domain to the intracellular CD3 $\zeta$ region of the TCR; was first described in the early $1990 \mathrm{~s}^{2}$. In vivo studies showed that first generation CAR T-cells exhibited limited proliferation, persistence and anti-tumour function ${ }^{6-8}$. The addition of an intracellular costimulatory domain within the CAR, positioned proximally to $\mathrm{CD} 3 \zeta$, led to the generation of second-generation CAR T-cells, which exhibit improved proliferation and anti-tumour function in vivo. In 2011, the simultaneous infusion of first and second-generation autologous CAR T-cell products was carried out in human patients, demonstrating that second generation CAR T-cells had significantly enhanced expansion and persistence in the peripheral blood ${ }^{9}$. Over the past 10 years, there has been a rapid increase in the number of CAR T-cell trials conducted. A simple search on clinicaltrials.gov using the search term 'chimeric antigen receptor' as of $29^{\text {th }}$ April 2020, shows 1429 registered studies. 
CAR T-Cell therapy has shown high efficacy in treating patients with relapsed and/or refractory (r/r) B-cell Non Hodgkin Lymphoma (B-NHL) with Overall Response (OR) rates reported at around $80 \%$, Complete Response (CR) rates at around $50 \%$ and durable progression free survival (PFS) seen in approximately $40 \%$ of patients ${ }^{10} 11$. These excellent response rates have led to two CAR T-cell therapies, tisagenlecleucel (CTL019, or Kymriah ${ }^{\circledR}$; Novartis) and axicabtagene ciloleucel (KTE-C19, or Yescarta ${ }^{\circledR}$; Kite Pharma), being licensed in the US, Europe and Australia for this indication ${ }^{12-14}$. These licensed CAR T-Cell therapies used for treatment of $\mathrm{r} / \mathrm{r}$ B-NHL are second generation CARs directed against the B-cell antigen CD19, an antigen expressed throughout B-Cell maturation and typically retained in B-cell malignancies such as B-NHL. ${ }^{15}$. However, with $\mathrm{CR}$ rates of around $50 \%$ and durable remissions seen in around $40 \%$ of patients, there is a need to improve upon these results with more effective CAR T-Cell therapies. Third-generation CARs contain two intracellular costimulatory domains have the potential to increase the efficacy of CAR T-Cell therapy ${ }^{16}$.

This research project has involved setting up and initiating a Phase 1 Investigator-Led Trial of a third generation anti-CD19 CAR T-Cell product against r/r B-NHL. This third generation CAR T-Cell product is named WZTL-002 and comprises autologous T-cells transduced to express an anti-CD19 CAR incorporating two costimulatory intracellular domains: the Toll/interleukin-1 receptor (TIR) domain of Toll Like Receptor 2 (TLR2) and CD28, alongside $\mathrm{CD} 3 \zeta$ at the cytoplasmic tail. A similar third generation CAR construct which contains the same costimulatory domains named '1928zT2', manufactured at the Guangzhou Institute of Biomedicine and Health (GIBH), showed enhanced in vivo and in vitro efficacy in preclinical studies in comparison to its second generation CAR counterpart ${ }^{17}$. A phase 1 dose escalation study of 1928zT2 CAR T-Cells in patients with r/r B-Cell Acute Lymphoblastic Leukaemia (B-ALL) has been carried out in Guangzhou, China (clinicaltrials.gov number, NCT02822326); a maximum tolerated dose was identified, and efficacy against extramedullary $\mathrm{CD} 19^{+}$tumours has been reported ${ }^{18}$, providing rationale to assess the efficacy of TLR2 TIR domain-incorporating third-generation CAR T-cells against r/r B-NHL. A number of changes were made to generate WZTL-002 in comparison to 1928zT2 CAR T-Cells. These differences are summarised as follows:

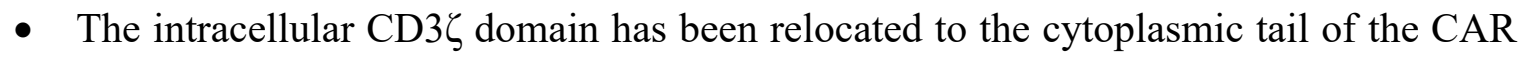
to improve function and comparability of clinical data with other second-and thirdgeneration CAR T-cell therapies. 
- A reporter gene in the CAR transgene construct encoding green fluorescent protein (GFP) has been removed to eliminate the possibility of anti-GFP immune responses.

- The manufacturing process has been modified in order to meet Good Manufacturing Practice (GMP) standards and Medsafe (harmonised with European) regulatory requirements; this includes upgrading to a third-generation lentiviral vector, using commercially-available GMP-grade products and minimising the use of animal-derived proteins in the manufacturing process.

Therefore, in view of these changes to the third generation CAR T-Cell product and targeting a different disease population (B-NHL instead of B-ALL), a Phase 1 dose escalation study has been set up and discussed in these thesis.

\section{Goals of Research Project:}

1) Literature Review: To conduct a literature review of CAR T-cell therapy in patients with B-NHL. This will comprise a review into the impact of costimulatory domains and CAR T-cell dose on clinical efficacy and toxicity, including reviewing the current data for third generation CAR T-cell constructs.

2) Study Design: Along with the study PI (Dr Robert Weinkove, Clinical Director of the Malaghan Institute) and study statistician, to work on and develop the study design (including dose escalation strategy; dosing cadence and definition of maximum tolerated dose).

3) Regulatory submissions for trial: In conjunction with the study PI, to prepare submissions to the Gene Technology Advisory Committee (GTAC), Health and Disability Ethics Committee (HDEC), Research Advisory Group Māori and Environmental Protection Agency (EPA) for the clinical trial. This includes writing the Investigator Brochure and Clinical Trial Protocol.

4) Risk mitigation strategies: Under supervision, to work with clinicians to develop risk mitigation strategies for key CAR T-cell toxicities, including cytokine release syndrome (CRS) and Immune effector cell associated neurotoxicity syndrome 
(ICANS). To design and implement a training programme for CRS and ICANS identification and management for nurses and clinicians, in conjunction with the PI and Study Nurse.

5) Study Conduct: In conjunction with the study PI and Study Nurse, to carry out study consent, screening procedures, subject enrolment and assess subjects before and after study treatment.

6) Data Analysis: To participate as a member of the Trial Management Committee (TMC), and review safety and efficacy data, which will also be reviewed by a Data Safety Monitoring Committee (DSMC).

The structure of this thesis is as follows:

Chapter 1: This chapter provides a brief introduction to the thesis

Chapter 2: This chapter provides a review of the anti-CD19 CAR T-cell therapy literature in patients with $\mathrm{r} / \mathrm{r}$ B-NHL. It provides context to the disease groups included within B-NHL and the current need for anti-CD19 CAR T-cell therapies for selected patients with this disease. The limitations of anti-CD19 therapy both in terms of efficacy and toxicity are discussed, along with the risk mitigation and management strategies for anti-CD19 CAR Tcell toxicities. The final section of this chapter which evaluates the functional and clinical impact of costimulatory domains with CAR T-cells, is taken from a published review article, for which I am a co-author. The sections of this review included in this thesis are ones in which I made significant contributions to as a co-author. I researched, designed and produced Figure 2.2 for this article.

The published review article which has been partly included within this section is: Selecting costimulatory domains for chimeric antigen receptors: functional and clinical considerations. Weinkove, R.; George, P.; Dasyam, N.; McLellan, A, D.; J Clin Transl Immunology, 2019 May 11;8(5):e1049 
Chapter 3: Chimeric antigen receptor T-cell therapies: Optimising the Dose.

Dasyam, N.; George, P.; Weinkove R.; Br J Clin Pharmacology, 2020 March 16. doi: $10.1111 / b c p .14281$

This chapter has been published. It reviews the literature around methods for CAR T-cell quantification, the pharmacokinetic properties of CAR T-cells and the influencing factors, optimising CAR T-cell dose in terms of minimising toxicity and maximising efficacy, design of CAR T-cell trial dose escalation trials and future perspectives around cost effectiveness of CAR T-cell therapy. My contribution to this article was toward all clinical components to the article including significant contributions to the following chapters: 'Factors influencing pharmacokinetics - lymphodepleting chemotherapy and tumour burden,' Drug interactions,' 'Dose exposure and dose-response relationships,' 'Dose toxicity relationship - can a therapeutic window be identified and risk-stratified dosing,' 'Dose escalation trials' and Beyond MTD.' I have added an additional sub-section to this chapter: Section 3.6.4.1 Dosing in anti-CD19 CAR T-cell trials registered on clinicaltrials.gov, which includes results from a search on CAR T-cell dosing in anti-CD19 CAR T-cells that I have carried out.

\section{Chapter 4: Chimeric antigen receptor T-cells in New Zealand: Challenges and} Opportunities

This chapter is a viewpoint article in advanced draft that is planned to be submitted to the New Zealand Medical Journal but has not been accepted for publication at the time of submitting this Masters Thesis. I will be the first author of this article when it is submitted to the New Zealand Medical Journal. This article outlines the regulatory landscape required for implementing CAR T-cell therapy in New Zealand based on experience from setting up the ENABLE trial, and also addresses important clinical policies and preparations which are recommended at CAR T-cell treating centres, before patients are treated with CAR T-cell therapy. I have been the primary writer of the following sections of the article: 'Leukocyte harvest and leukapheresis,' 'Cultural considerations,' 'Clinical delivery of CAR T-cell therapy - CAR T-cell toxicity working group, CAR T-cell toxicity education, Pharmac provision of tocilizumab, Patient education and International cellular therapies registry.' 
Chapter 5: Third-generation anti-CD19 chimeric antigen receptor T-cells incorporating a TLR2 domain for relapsed or refractory B-cell lymphoma: a phase I clinical trial protocol (ENABLE).

George, P.; Dasyam, N.; Giunti, G et al. BMJ Open, 2020 Feb, 10(2): e034629. doi: 10.1136/bmjopen-2019-034629

This chapter has been published in BMJ Open as a protocol paper. It outlines the protocol for the ENABLE Phase 1 anti-CD19 CAR T-cell trial. As the first author of this manuscript, I was the primary writer of all sections within this chapter. For the ENABLE trial protocol itself, which this manuscript describes, outlines and contextualises; I was the primary author together with my supervisor, Dr Robert Weinkove. In addition to the published manuscript, I have included an additional figure (

Figure 5.2), which I have constructed to illustrate the ' $3+3$ ' dose escalation design adopted in the ENABLE trial.

Chapter 6: Preliminary ENABLE trial experience

This chapter outlines the preliminary experience from the ENABLE Trial Management Committee, a case history from the first participant treated on the ENABLE trial and summarises initial safety and pharmacokinetic data obtained from this participant.

Chapter 7: Discussion

This chapter provides a commentary on what preparing and setting up the ENABLE trial in a New Zealand regulatory environment has involved. It discusses my findings on what CAR T- 
cell therapy entails from a regulatory and clinical perspective and provides insight on the potential significance of this study. Finally, it discusses the possible future directions for antiCD19 CAR T-cell therapy for the treatment of B-cell lymphoma.

\section{Appendices:}

Appendix 1 and 2 are the Quicksheets produced in preparation for delivering CAR T-cell treatment in Wellington Hospital that provide guidance on the recognition and management of Cytokine Release Syndrome (CRS) and Immune Effector Cell Encephalopathy Syndrome (ICANS).

Appendix 3: Provides the references for the additional published and as yet unpublished manuscripts contributed to during this Masters thesis. 


\section{Literature Review}

\subsection{B-cell non-Hodgkin lymphoma}

\subsubsection{Incidence, pathogenesis and principal subtypes}

Non-Hodgkin lymphomas (NHLs) are the $7^{\text {th }}$ most common malignancies diagnosed worldwide: around $2 \%$ of people in higher income countries develop an NHL in their lifetimes, and 231,0000 deaths were attributed to NHL in 2015 ${ }^{19}$. Between 2005 and 2015, global NHL registrations rose by $56 \%$, due to a combination of population growth, aging populations and rising age-specific incidence ${ }^{19}$. Over $90 \%$ of NHL arise from Blymphocytes, leading to a diagnosis of B-cell Non Hodgkin Lymphoma (B-NHL) ${ }^{20}$.

B-NHLs are a heterogenous group of malignancies which include indolent, slow-growing malignancies such as follicular lymphoma, and more aggressive, faster-growing malignancies such as diffuse large B-cell lymphoma (DLBCL) and Burkitt lymphoma (BL).

\subsubsection{Diffuse large B-cell lymphoma}

DLBCL is the most common subtype of B-NHL and accounts for approximately $40 \%$ of the global lymphoma burden ${ }^{21}$. DLCL is an aggressive form of B-NHL but there is significant pathogenetic and biological heterogeneity in this disease ${ }^{22}$. Most DLBCLs derive from the germinal centre where B-cells undergo proliferation due to T-cell dependent antigen stimulation leading to somatic hypermutation of the variable region of the immunoglobulin gene in proliferating centroblasts ${ }^{22}$. Other DLBCL subtypes based on the putative cell of origin include activated B-cell like (ABC) DLBCL, resembling features of plasmablastic Bcells committed to terminal differentiation and Primary Mediastinal B-cell Lymphoma 
(PMBCL), most likely arising from thymic B-cells ${ }^{22}{ }^{23}$. The 2016 World Health Organisation (WHO) classification recognises a number of entities that can be considered variants of DLBCL, and which despite distinct clinical presentations and histological features, are typically treated similarly to DLBCL. These include: intravascular large B-cell lymphoma; Tcell/histiocyte rich large B-cell lymphoma; DLBCL associated with chronic inflammation; primary cutaneous DLBCL, leg type; and $\mathrm{EBV}^{+} \mathrm{DLBCL}^{24}$.

\subsubsection{Other Aggressive B-cell lymphomas}

There are a number of other histological subtypes of aggressive (high-grade) mature B-cell lymphomas. BL is a highly aggressive form of B-NHL that accounts for less than $5 \%$ of adult B-NHL cases $^{25}$. BL cells are derived from mature germinal or post germinal centres and occurs due to rearrangements of the MYC oncogene leading to overexpression of c-myc and a rapidly growing tumour with a doubling time of 24 to 48 hours $^{25}$. There are now two defined histological subtypes of aggressive B-cell lymphoma that fit within the spectrum between DLBCL and BL as per the WHO classification of lymphoid neoplasms ${ }^{24}$. These subtypes are High grade B-cell lymphomas with MYC and BCL2 and/or BCL6 rearrangements, and High grade B-cell lymphoma Not Otherwise Specified (NOS). The former diagnosis is made irrespective of morphological findings, whereas the latter diagnosis is made when there is high grade morphology with features intermediate between DLBCL and BL or blastoid morphology, but lack gene translocations of MYC, BCL2 and/or BCL6 ${ }^{24}$.

\subsubsection{Follicular Lymphoma}

Follicular lymphoma (FL) is the most common indolent subtype of B-NHL and accounts for approximately $35 \%$ of all NHL $\operatorname{cases}^{26}$. FL lymphoid tissue comprises proliferation of malignant germinal centre B-cells, mainly comprising of centrocytes and centroblasts and around $85 \%$ of FL cases contain a $\mathrm{t}(14 ; 18)$ translocation leading overexpression of the antiapoptotic protein $\mathrm{bcl} 2^{26}$. Histopathological grading, as per WHO criteria is determined by the number of centroblasts per high powered field and is graded from 1-3B. Grades $1-3 \mathrm{~A}$ are usually considered low grade (although grade 3A FL has been excluded from several low grade B-NHL clinical trials), and grade $3 \mathrm{~B}$ is regarded and treated as a high grade B-NHL ${ }^{27}$. 


\subsubsection{Mantle Cell Lymphoma}

Mantle Cell Lymphoma (MCL) is a relatively rare and incurable subtype of B-NHL and is characterised by a CCNDN1/IGH translocation leading to constitutive overexpression of Cyclin D1 and cell cycle dysregulation ${ }^{28}$. MCL is a heterogeneous disorder and there are two distinct pathogenetic types of MCL. The first type is classical MCL, comprising mature Bcells do not enter the germinal centre, have no or minimal mutations in $I G H V$ and express transcription factor SOX $11^{24}$. Typically, patients with classical MCL present with a more aggressive, nodal presentation ${ }^{28}$. The second less common type is leukaemic, non-nodal MCL, comprising B-cells that pass through the germinal centre with $I G H V$ somatic hypermutation and no or minimal SOX11 expression ${ }^{24}$. Patients with leukaemic, non-nodal MCL typically have a more indolent presentation with disease involvement in the peripheral blood, bone marrow and spleen, although secondary genetic abnormalities such as TP53 mutations can result in a more aggressive disease phenotype ${ }^{28}$.

\subsubsection{Transformed Indolent Lymphoma}

Indolent B-NHL subtypes, most frequently follicular lymphoma, can transform to a more aggressive lymphoma resembling DLBCL, with development of clinical and histological features of aggressive B-NHL, an increase in proliferation rate, and acquisition of additional genetic changes with an estimated progression risk of 2-3 percent per year. ${ }^{26}$

\subsubsection{Treatment}

\subsubsection{Aggressive B-Cell Lymphomas}

First-line chemoimmunotherapy for DLBCL, comprising the chimeric monoclonal antiCluster of Differentiation 20 (CD20) antibody rituximab together with combination chemotherapies such as Cyclophosphamide, Hydroxydaunorubicin, Oncovin (vincristine) and Prednisone (CHOP); typically results in a cure in around $60-70 \%$ patients depending on disease stage, DLBCL subtype, molecular risk factors and patient characteristics ${ }^{29}$. However, in around $20 \%$ of patients, DLBCL fails to respond completely to initial chemoimmunotherapy, and in a further $20 \%$ relapse occurs after a prior complete response ${ }^{30}$. 
Patients diagnosed with High grade B-cell lymphoma with $M Y C$ and BCL2 and/or BCL6 rearrangements (ie Double Hit (DH) or Triple Hit (TH) Lymphoma), have inferior outcomes when treated with standard front line chemoimmunotherapy regimens for DLBCL such as R$\mathrm{CHOP}^{31}$. In this setting, intensified initial chemoimmunotherapy therapy regimens such as dose-adjusted R-EPOCH or R-CODOX-M/R-IVAC are often adopted ${ }^{31}$. Although randomised data are lacking in this area, one single arm, prospective study using DA-EPOCH-R in myc rearranged aggressive B-NHL, reported an Event Free Survival (EFS) of $73.4 \%$ in the sub group of patients with $\mathrm{DHL}^{32}$.

\subsubsection{Indolent B-cell Lymphomas}

Indolent B-NHLs such as FL requiring treatment typically respond well to to first line chemoimmunotherapy regimens incorporating anti-CD20 monoclonal antibodies such as RCHOP, RCVP and R-Bendamustine, although treatment is not curative and the disease typically follows a relapsing and remitting course ${ }^{33}$. With standard chemoimmunotherapy regimens in Follicular NHL, five year Progression Free Survival (PFS) and five year Overall Survival (OS) rates are reportedly between 56-66\% and $82-85 \%$ respectively, although there are a subset of patients who do poorly and relapse within 24 months of initial therapy ${ }^{27}$. In patients with Follicular NHL who achieve a Partial Response (PR) or Complete Response (CR) after initial treatment with chemoimmunotherapy, the addition of two monthly maintenance rituximab for two years has been found to significantly improve PFS irrespective of baseline factors such as age and prognostic score at diagnosis, although no significant improvement in OS has been observed to date ${ }^{27}$.

In MCL, younger patients are often treated with intensified immuno-chemotherapy regimens incorporating high dose cytarabine such as the Nordic regimen or Hyper-CVAD, followed by an autologous stem cell transplant (Auto SCT). Following this approach, a median PFS of over seven years has been reported ${ }^{34}$.

\subsubsection{Management of relapsed and refractory disease}




\subsubsection{Aggressive B-Cell Lymphomas}

Patients with DLBCL that do not respond to first line chemoimmunotherapy, or who relapse after chemoimmunotherapy, are typically treated with 'salvage' chemotherapy regimens followed by myeloablative chemotherapy and an Auto SCT. This typically leads to long-term PFS rates of $30-40 \%{ }^{35}$.

Among patients with DLBCL that is either refractory to salvage chemotherapy regimens, or relapses early (within 12 months) following an autograft, outcomes are dismal ${ }^{21}$. The SCHOLAR-1 Study, which looked at pooled data from 636 participants in cohort studies and two large phase 3 randomised control trials; reported a pooled OR rate of $26 \%$, a pooled CR rate of 7\%, a median OS of less than 10 months, and long term survival was achieved in fewer than $20 \%$ of patients ${ }^{36}$. Therefore there is a significant unmet clinical need in patients with $\mathrm{r} / \mathrm{r}$ aggressive B-NHL.

In patients with $\mathrm{r} / \mathrm{r} \mathrm{DH}$ or TH lymphoma after intensive induction chemotherapy, outcomes are dismal $^{37}$. In one retrospective study looking at outcomes in r/r DLBCL post Auto SCT, those patients with DH lymphoma had a four year PFS of $28 \%$ and a four year OS of $25 \%{ }^{37}$. The management of patients with $\mathrm{r} / \mathrm{r}$ DH or TH Lymphoma is clearly an area of unmet clinical need and new treatment approaches are required to improve long term outcomes ${ }^{31}$.

In patients with transformed FL that have received previous therapies, the 5 year and 2 year OS rates have only been reported at $21 \%$ and $35 \%$ respectively ${ }^{38}$. Allogeneic stem cell transplants (Allo-SCTs) in transformed FL is associated with a high non-relapse mortality of around $40 \%$ within the first year post transplant and poor five year OS rates around $22 \%$ are reported $^{39}$. Relapse of transformed FL after Allo-SCT is associated with a dismal prognosis ${ }^{40}$.

\subsubsection{Indolent B-cell Lymphomas}

In patients with FL at first relapse, treatment with an alternative rituximab containing immuno-chemotherapy regimen is often an effective treatment strategy with high response rates but less durable responses seen in comparison to front line treatment ${ }^{41}$. In patients with primary refractory FL or those who relapse or progress within 24 months of primary therapy, the outcomes are much poorer, with OS rates of $26-46 \%$ at five years, when treated with 
current available therapies ${ }^{42}$. Auto and Allo-SCTs are often recommended for fitter patients with $\mathrm{r} / \mathrm{r} \mathrm{FL}^{43}$, the higher non-relapse mortality of Allo-SCT being offset by lower relapse rates than Auto-SCT ${ }^{44} 45$. Among individuals with relapsed FL who relapse after, or are ineligible for, Allo-SCT, prognosis is extremely poor ${ }^{46} 47$.

In patients with MCL who relapse following an Auto-SCT, management options are limited $^{48}$. In this scenario, an Allo-SCT is a therapeutic option with OS rates of $38-64 \%$ reported at two years post-transplant ${ }^{49-50}$. Among individuals with relapsed MCL who relapse after, or are ineligible for, allogeneic stem cell transplantation, the prognosis is very poor ${ }^{47}$.

\subsection{Chimeric antigen receptor T-cells}

\subsubsection{History}

Adoptive Cell Therapy (ACT) involves the isolation of tumour specific T-cells, laboratory modification and expansion, then re-injection of the modified T-cells back into the patients' circulation $^{51}$. Autologous T-lymphocytes have been used as adoptive cell therapies for a number of years, with clinical responses observed in cancer patients treated with tumourinfiltrating lymphocytes in the $1980 \mathrm{~s}^{52} 53$. In 1989, the genetic modification of T-cells to direct their specificity in a non-major histocompatibility complex (MHC) restricted manner, by generating chimeric TCR genes composed of the constant domains of the T-cell receptor gene fused to the variable domains of an antibody encoding gene, to target cancer cells, was reported ${ }^{3}$.

In 1993, non-MHC restricted lysis of target cells was seen using T-cells transduced to express chimeric genes composed of a scFv of an antibody linked with the constant regions of the TCR chains, thereby forming a CAR T-cell ${ }^{2}$. These first reports of CAR T-cells, allowed ACT to target tumours previously not thought possible, bypassing the need for interaction between the TCR and antigenic peptide in complex with MHC molecules ${ }^{54}$.

A further discussion of the history of CAR T-cells is provided in Chapter 3.2.1 History of CAR T-cells. 


\subsubsection{Genetic Transduction in CAR T-cell therapy}

Expression of the CAR on the T-cell surface is usually achieved by incorporating a gene encoding the CAR construct into T-cell genomes using a retroviral vector (a replication incompetent lentivirus or $\gamma$-retrovirus) $)^{5}$. Non viral transduction systems such as the transposon/transposase system, have also been used to generate CAR T-cells used in some clinical trials ${ }^{5556}$. Both lentiviruses and $\gamma$-retrovirus are derived from the human immunodeficiency virus (HIV), a member of the retrovirus family and have been adapted as clinical gene transfer vectors in CAR T-cell therapy. These retroviral vectors are packaged into separate plasmids, with much of the viral genome replaced with the transgene of interest encoding the CAR construct, which is integrated into the T-cell genome on transduction. Successive generations of lentiviral (LV) vectors have incorporated enhanced safety features to reduce the risk of recombination events to generate replication competent lentiviruses ${ }^{57}$. Accumulated laboratory and clinical experience indicates the latest generation of LV vectors (third generation), are extremely unlikely to become replication competent and recombination events that restore replication competency have never been reported in this setting ${ }^{58}$.

Furthermore, the risk of insertional mutagenesis, whereby genetic material from the vector is inserted in close proximity to proto-oncogenes in the target cell genome, is thought to be low using LV vectors ${ }^{59}$. There have been no reported cases of vector-associated leukaemogenesis in CAR T-cell trials to date $e^{60-61}$.

\subsubsection{First and second generation CAR T-cells}

The first generation of CAR T-cells combined an extracellular antigen-binding scFv and an

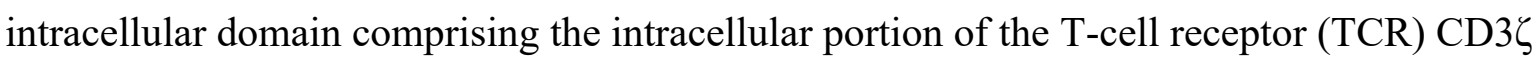
subunit $^{2}$. Despite T-cell activation in response to antibody dependent recognition of a target antigen, first generation CAR T-cells were found to have limited proliferation and antitumour efficacy in vivo ${ }^{6}$. The addition of an intracellular costimulatory domain proximal to $\mathrm{CD} 3 \zeta$ such as CD28 or 4-1BB, was found to significantly enhance T-cell expansion and antitumour efficacy in response to CAR binding to its cognate antigen ${ }^{62}$. The superior CAR Texpansion of second generation CAR T-cells containing the CD28 costimulatory domain, was demonstrated Savoldo et al, when six patients with lymphoma were co-infused with first and second generation CAR T-cells; in all six patients expansion of the second generation CAR T-cells was greater than the first generation CARs ${ }^{9}$. In light of these results, the 
majority of CAR T-cell trials to date have used second generation CAR T-cells (see Figure 2.2) and both of the commercially licensed anti-CD19 CAR T-cell products, axicabtagene ciloleucel and tisagenlecleucel, are second generation products (see Figure 2.1) ) $^{63}$.

\subsubsection{Lymphodepleting chemotherapy}

The use of lymphodepleting chemotherapy before CAR T-cell administration is considered critical for robust CAR T-cell expansion and persistence; and necessary to induce treatment responses $^{65-66}$. The most frequently used regimens comprise fludarabine with cyclophosphamide, typically fludarabine $25-30 \mathrm{mg} / \mathrm{m}^{2} / \mathrm{d}$ and cyclophosphamide $250-500$ $\mathrm{mg} / \mathrm{m}^{2} / \mathrm{d}$ for three consecutive days ${ }^{1}$. This combination of chemotherapy is recommended prior to administration of both of the commercially licensed CAR T-cell therapies, Axi-cel and Tisagenlecleucel ${ }^{6364}$. The role of lymphodepleting chemotherapy before CAR T-cell administration is discussed further in Chapter 3.4.1.1 Lymphodepleting chemotherapy.

\subsubsection{Anti-CD19 CAR T-cells}

CD19 is a $95 \mathrm{kDa}$ transmembrane glycoprotein of the immunoglobulin (Ig) superfamily and is expressed on B-cells throughout maturation from pre-B stage lymphoblasts to mature B-cells 67. The majority of clinical success in CAR T-cell therapies to date has been targeting CD19 in patients with B-cell malignancies, including B-ALL and B-NHL ${ }^{68}$. There are a number of likely reasons for the success for anti-CD19 CAR T-cell therapy in the treatment of B-cell malignancies. Firstly, CD19 is often highly expressed in B-cell cancers, providing a good target for tumour killing ${ }^{69}$. Secondly, although CD19 is also required for normal B-cell development, it is not expressed outside the B-cell lineage ${ }^{70}$. Depletion of healthy B-cells following anti-CD19 CAR T-cell treatment, often leads to B-cell aplasia and hypogammaglobulinaemia; but this can generally be successfully managed with intravenous immunoglobulin replacement ${ }^{70} 71$. The absence of CD19 expression on other human cells, reduces the severity of on target, off tumour effects, thereby reducing potentially fatal toxicity ${ }^{68}$. Lastly, in B-cell cancers such as B-ALL, the immunosuppressive tumour microenvironment is less of an inhibitory factor to CAR T-cell activation in response to CD19 recognition and binding, in comparison to solid organ tumours ${ }^{72}$. 
Autologous anti-CD19 CAR T-cell therapy has shifted the treatment paradigm for paediatric and young adult patients with r/r B-ALL ${ }^{73}$. Results from anti-CD19 CAR T-cell therapy trials in patients with $\mathrm{r} / \mathrm{r}$ B-ALL have reported CR rates between 68 and $93 \%{ }^{74}$. Kymriah ${ }^{\circledR}$ (tisagenlecleucel), an autologous commercial second generation anti-CD19 CAR T-cell product, is now approved by the Food and Drug Administration (FDA), EMA (European Medicines Agency) and the Therapeutic Goods Association (TGA) in Australia, for the treatment of paediatric and young adult patients up to 25 years of age with B-cell precursor ALL that is refractory, in relapse post-transplant, or in second or later relapse ${ }^{1364}$.

Figure 2.1 First, second and third generation CARs. Figure adapted from Maus et $\mathrm{al}^{75}$

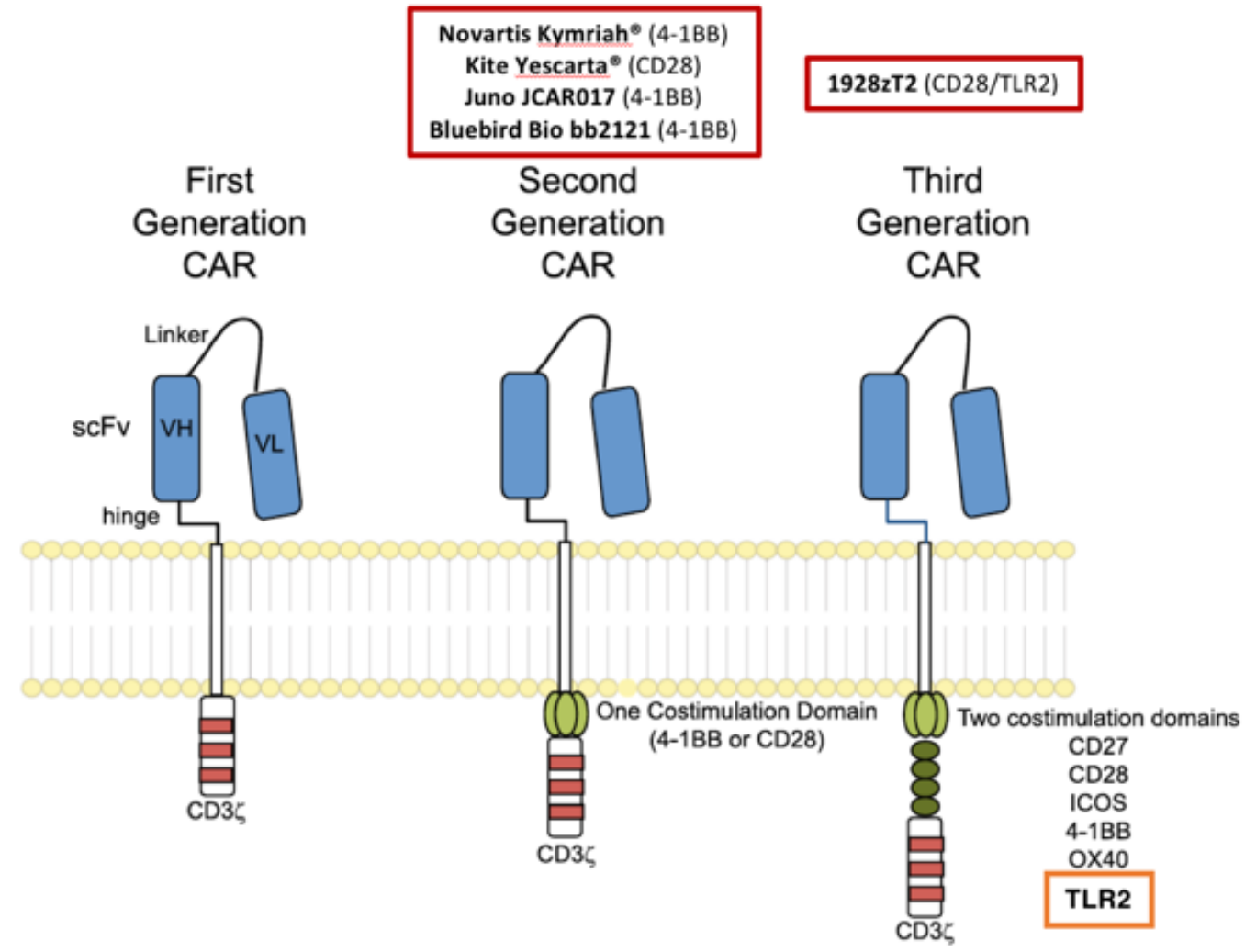

\subsection{Anti-CD19 CAR T-cell therapy in r/r B-NHL}

The clinical outcomes for anti-CD19 CAR T-cell therapies in $r / r$ B-NHL are summarised in Table 2.1, which includes reported data from the three pivotal anti-CD19 CAR T-cell trials in r/r B-NHL: the ZUMA-1 Study, the JULIET Study and the TRANSCEND NHL 001 Study $^{11}$ 
7677. At the time of writing, two of the second generation anti-CD19 CAR T-cell products tested in these three pivotal trials, axi-cel and tisagenlecleucel, have been licensed in the US and in Europe for the treatment of r/r DLBCL, with tisagenlecleucel also licensed for this indication in Australia ${ }^{13} 146364$. The clinical outcomes reported for patients with $\mathrm{r} / \mathrm{r}$ B-NHL treated with the anti-CD19 CAR T -cell products tested in these three pivotal trials, axi-cel, tisagenlecleucel and lisocabtagene maraleucel (liso-cel); are discussed in this section. The majority of patients treated in these pivotal trials had a diagnosis of $r / r$ DLBCL, a minority of patients had transformed FL or PMBCL, but all patients had high grade disease. Therefore, early anti-CD19 CAR T-cell clinical trial data outside of these three pivotal trials in $\mathrm{r} / \mathrm{r}$ indolent B-NHL, including FL and MCL, will also be discussed in this section.

\subsubsection{Axicabtagene cilolecleucel}

The multicentre phase 2 ZUMA-1 trial enrolled 111 individuals with $\mathrm{r} / \mathrm{r}$ aggressive B-NHL and administered the anti-CD19 CAR T-cell product, axi-cel to 101 patients $^{11}$. Axi-cel is an autologous second generation anti-CD19 CAR product containing the CD28 costimulatory domain. It is manufactured from autologous T-cells harvested by leukapheresis, which are then activated and transduced using a retroviral vector containing an anti-CD19 CAR gene. ${ }^{11}$

Reasons for not administering axi-cel to 10 patients included unsuccessful CAR T-cell product manufacture, adverse events and disease progression. Out of 101 patients treated, 77 had a diagnosis of DLBCL, 16 had a diagnosis of transformed FL and 8 had a diagnosis of PMBCL. The median age was 57 years, $88 \%$ of patients had received $\geq$ three prior therapies and $21 \%$ of patients had relapsed after prior Auto-SCT. Patients with central nervous system (CNS) involvement or prior Allo-SCT were ineligible. Bridging therapy between leukapheresis and axi-cel infusion was not permitted and all patients received lymphodepleting chemotherapy comprising $500 \mathrm{mg} / \mathrm{m}^{2}$ of cyclophosphamide and $30 \mathrm{mg} / \mathrm{m}^{2}$ of fludarabine per day for three days, followed by a single infusion of Axi-cel 48 hours later, at a dose of $2 \times 10^{6} \mathrm{CAR}$-positive $\mathrm{T}$ cells $/ \mathrm{kg}$.

The OR rate was $83 \%$, and CR rate was $55 \%{ }^{11}$. After a median follow up of 27.1 months, the median PFS was 5.9 months, the median duration of response was 11.1 months and the median OS was not reached ${ }^{78}$. The Durability of response was highly dependent on the depth of response with those achieving a $\mathrm{CR}$ at three months having a high chance of remaining 
progression free at two years, with an estimated PFS of $72 \%{ }^{78}$. Conversely, those with stable disease at three months were found to have an estimated two year PFS of $22.2 \%{ }^{78}$. Long term follow up of patients treated with axi-cel on the ZUMA-1 trial reported by Locke et al, estimated a 24 -month survival proportion of 50.5\%; this represents a significant improvement to the long term survival rates of less than $20 \%$ reported in the SCHOLAR-1 Study, in patients with $\mathrm{r} / \mathrm{r} \mathrm{DLBCL}^{36} 78$.

Recent retrospectively analysed real world data in patients with $\mathrm{r} / \mathrm{r}$ B-NHL who received axicel in a standard of care setting, reported similar response rates to the ZUMA-1 study despite a significant proportion of patients not meeting eligibility criteria for this study ${ }^{79}$.

\subsubsection{Tisagenlecleucel}

Results from the multicentre Phase II JULIET Study reporting outcomes of individuals with $\mathrm{r} / \mathrm{r}$ aggressive B-NHL treated with tisagenlecleucel, have been published ${ }^{76}$. Tisagenlecleucel is a second generation anti-CD19 CAR T-cell product containing the $41 \mathrm{BB}$ costimulatory domain. It is manufactured from autologous T-cells harvested by leukapheresis, which are then activated and transduced using a lentiviral vector containing an anti-CD19 CAR gene ${ }^{76}$.

In the JULIET study, 111 out of 165 enrolled patients (67\%) received treatment with tisagenlecleucel. Reasons for not receiving tisagenlecleucel infusion included disease progression precluding treatment or leading to patient death, unsuccessful CAR T-cell manufacture and other adverse events ${ }^{76}$. Out of 111 patients treated, 88 had a diagnosis of DLBCL, 21 had transformed FL and two had a diagnosis termed 'other.' The median age was 56 years, $52 \%$ of patients had received $\geq$ three prior therapies, $49 \%$ of patients had received a previous Auto-SCT and 27\% patients had DH or TH lymphoma ${ }^{76}$. Patients with CNS involvement or prior Allo-SCT were ineligible. 92\% of patients received bridging therapy between leukaphereses and tisagenlecleucl infusion. Lymphodepleting chemotherapy was given (either cyclophosphamide $250 \mathrm{mg} / \mathrm{m}^{2}$ and fludarabine $25 \mathrm{mg} / \mathrm{m}^{2}$ for three days or bendamustine $90 \mathrm{mg} / \mathrm{m}^{2}$ for two days) prior to administering a dose of $0.1-6.0 \times 10^{8}$ total antiCD19 CAR T-cells ${ }^{76}$.

Among 93 patients reported in the efficacy analysis, this study reported OR and CR rates of $52 \%$ and $40 \%$ at three months post treatment respectively ${ }^{76}$. The median OS among all 
treated patients was 12 months and approximately $35 \%$ of all treated patients remained progression free at 12 months. Among patients that achieved at PR or CR at three months post treatment, durable response were observed with $83 \%$ of these patients reported to be progression free at 12 months. In patients with $\mathrm{DH}$ lymphoma, the OR rate and $\mathrm{CR}$ rate were $50 \%$ and $25 \%$.

\subsubsection{Lisocabtagene maraleucel}

Results from the open label, multi-centre Phase 1 trial TRANSCEND NHL 001, have been reported in abstract form at the American Society of Haematology Conference in November $2019^{77}$. In this trial, liso-cel, a second generation anti-CD19 CAR T-cell product containing the $41 \mathrm{BB}$ costimulatory domain was tested in patients with $\mathrm{r} / \mathrm{r}$ B-NHL. Liso-cel is manufactured using a lentiviral vector to insert an anti-CD19 CAR gene into harvested autologous T-cells and uniquely is comprised of a fixed 1:1 composition of CD4+ and CD8+ CAR T-cells ${ }^{80}$.

In total, 344 patients underwent leukapheresis, 269 patients received treatment with liso-cel conforming to product standards and 256 patients were included in the efficacy set ${ }^{77}$. Out of 269 patients, 137 had DLBCL and 132 had other diagnoses including transformed FL, HGBCL and PMBCL. The median age was 63 years and the median number of prior therapies was three. A total of 33\% of patients had received a prior Auto-SCT and 3\% a prior Allo-SCT. Three percent of patients had secondary CNS lymphoma. Bridging therapy was permitted after leukapheresis and lymphodepletion with fludarabine $\left(30 \mathrm{mg} / \mathrm{m}^{2}\right)$ and cyclophosphamide $\left(300 \mathrm{mg} / \mathrm{m}^{2}\right.$ ) for three days was administered prior to liso-cel infusion. Liso-cel was tested across three dose levels, with $1 \times 10^{8}$ viable total CAR+ T-cells as the target level for dose confirmation.

After a median follow up of 12 months, an OR rate of $73 \%$ and a CR rate of 53\% was reported. The 12 month PFS was $44.1 \%$ and the 12 month OS was 57.9\%. The median OS was 21.1 months but was not reached in patients with achieving a CR. Subgroup analysis by histology showed patients with PBMCL and transformed FL had a higher PFS compared to patients with DH or TH lymphoma, DLBCL NOS, or HGBCL. 


\subsubsection{Anti-CD19 CAR T-cells in indolent B-NHL}

\subsubsection{Follicular Lymphoma}

One of the first case reports of anti-CD19 CAR T-cell efficacy by Kochenderfer et al at the National Cancer Institute (NCI) in the US, was in a patient with multiply relapsed $\mathrm{FL}^{81}$. A subsequent study from the NCI published in 2017, reported CRs in two patients with $\mathrm{r} / \mathrm{r}$ FL treated with second generation anti-CD19 CAR T-cells containing the CD28 costimulatory domain $^{82}$. Schuster et al reported results of 28 patients with $\mathrm{r} / \mathrm{r}$ B-NHL treated with tisagenlecleucel, 14 of which had $\mathrm{FL}^{83}$. Out of the 14 patients with FL, the median number of prior therapies was five, $86 \%$ had advanced stage disease, and $25 \%$ had received a prior Auto or Allo-SCT. In these 14 patients with FL, the OR rate was $89 \%$ and the CR rate was $71 \%$ and at a median follow up of 28.6 months, $89 \%$ of patients that achieved a response had maintained the response ${ }^{83}$. Subsequently in 2019, Hirayama et al reported results of 8 patients with $\mathrm{r} / \mathrm{r}$ FL and 13 patients with $\mathrm{r} / \mathrm{r}$ transformed FL treated with second generation anti-CD19 CAR T-cells containing the 4-1BB costimulatory domain, formulated in a 1:1 $\mathrm{CD}^{+}: \mathrm{CD}^{+} \mathrm{CAR} \mathrm{T}$-cell ratio ${ }^{84}$. Out of the FL patients, the median number of prior treatments was $4,75 \%$ had progressive disease after the last line of therapy, 50\% had failed previous Auto or Allo-SCT and 75\% had intermediate or high FL International Prognostic Index (FLIPI) score. The CR rate for patients with FL was $88 \%$ and all patients who achieved CR remained in remission at a median follow up of 24 months, comparing favourably to a CR rate of $46 \%$ and median duration of response of 10.2 months in patients with transformed $\mathrm{FL}^{84}$. However, it should be noted that in the study published by Hirayama et al, the majority of patients with FL (62\%) received a lymphodepleting chemotherapy regimen which contained approximately double the amount of cyclophosphamide compared to the regimen used the transformed FL cohort; thereby potentially contributing to the higher CR rate observed in patients with $\mathrm{FL}^{85}$. Accepting that results from a small number of patients with FL have been reported to date, there is increasing evidence that anti-CD19 CAR T-cell therapy may be a highly efficacious option for patients with $r / r$ FL.

\subsubsection{Mantle Cell Lymphoma}

Two early phase anti-CD19 CAR T-cell trials reported results in a limited number of patients with $\mathrm{r} / \mathrm{r}$ MCL with mixed results ${ }^{82} 86$. Turtle et al reported disappointing results in four patients with $\mathrm{r} / \mathrm{r}$ MCL treated with 4-1BB containing anti-CD19 CAR T-cells administered in 
a defined $1: 1 \mathrm{CD}^{+}: \mathrm{CD}^{+} \mathrm{CAR} \mathrm{T}$-cell ratio, with an OR rate of $25 \%$ and $0 / 4$ patients achieving a $\mathrm{CR}^{86}$. In 2017, Kochenderfer et al reported a complete response in the only patient with MCL treated with CD28 containing second generation anti-CD19 CAR T-cells in an early phase NCI trial ${ }^{82}$. However, results from the multicentre, phase 2 ZUMA-2 trial, have recently been published in the New England Journal of Medicine, showing efficacious results of KTE-X19 CAR T-cell therapy, an analogous product to axi-cel, in patients with r/r $\mathrm{MCL}^{87}$. Wang et al report that KTE-X19 was manufactured for 71 of 74 enrolled patients and administered to 68 of them. A primary efficacy analysis was performed on the first 60 patients treated with KTE-X19, reporting an OR rate of $93 \%$ and CR rate of $67 \%$. Out of 60 patients, the median number of prior therapies was three, 17\% of patients had TP53 mutations, $31 \%$ had blastoid morphology and $100 \%$ of patients were relapsed following or refractory to Btk inhibitor therapy. At 12 months the PFS rate was $61 \%$ and the OS rate was $83 \%$. At a median follow up of 12.3 months, $57 \%$ of all patients in the primary efficacy analysis and $78 \%$ of patients that achieved a $\mathrm{CR}$, continued to have a response, suggesting that durable responses could be achieved. A high percentage of patients with poor risk disease features such as a Ki-67 $\geq 50 \%$, blastoid morphology or a TP53 mutation, achieved an objective response, suggesting that anti-CD19 CAR T-cell therapy may benefit patients with prognostic features that typically fare poorly using other treatment options ${ }^{87}$. These results suggest that anti-CD19 CAR T-cell therapy may be an effective treatment option in $\mathrm{r} / \mathrm{r}$ $\mathrm{MCL}^{87}$.

\subsubsection{Summary}

There are no head to head comparisons of the three anti-CD19 CAR T-cell products tested in the pivotal trials ZUMA-1, JULIET and TRANSCEND NHL 001. However, the activity of all three CAR T cell products are similar in the separate phase I/II trials and in real world data for $\mathrm{r} / \mathrm{r}$ B-NHL, with durable responses seen in between 35 and $45 \%$ of patients including those in high-risk subsets such as DH Lymphoma ${ }^{88}$. It should be noted that the three CAR Tcell products have differences in the leukapheresis process, CAR T-cell product manufacturing method, use of bridging therapy, eligibility criteria and toxicity profile (see 2.5 Anti-CD19 CAR T-cell therapy toxicities).

Preliminary data on the use of anti-CD19 CAR T-cell therapy in $r / r$ indolent B-NHL shows promising efficacy and further trial results are awaited. Recent real world data presented from 
National Health Services England (NHSE) in patients with $\mathrm{r} / \mathrm{r}$ high grade B-cell lymphoma, demonstrated inferior outcomes compared to the pivotal trials ${ }^{89}$. At three months following CAR T-cell infusion, an ORR (PR or CR) of 37\% (21\% CR) was reported in 56 patients treated with axi-cel and an ORR of $29 \%(17 \% \mathrm{CR})$ was reported in 24 patients treated with tisagenlecleucel, with a median EFS of 3.1 months ${ }^{90}$. This was despite similar baseline characteristics as seen in the pivotal Phase II trials, reported by the UK national CAR-T selection panel. The cause for these differences is unclear and may be due to patient population \& selection, apheresis cell product or CAR-T product quality. Further collection of real-world data is needed in patients with $\mathrm{r} / \mathrm{r}$ high grade B-cell lymphoma, to assess contributory factors to the differences in response rates observed and to identify possible biomarkers that may be predictive of response. 
Table 2.1 Clinical outcomes for second generation anti-CD19 CAR T-cells for r/r B-NHL

\begin{tabular}{|c|c|c|c|c|c|}
\hline Study & $\begin{array}{l}\text { B-NHL } \\
\text { subtypes (n) }\end{array}$ & $\begin{array}{l}\text { Co-stimulatory } \\
\text { domain }\end{array}$ & Lymphodepletion & $\begin{array}{l}\text { ORR } \\
\text { n/total (\%) }\end{array}$ & $\begin{array}{l}\text { CR } \\
\text { n/total (\%) }\end{array}$ \\
\hline Sayoldo, 2011 & DLBCL (3), TFL (2), SLL (1) & CD28* & None & $0 / 6(0 \%)$ & $0 / 6(0 \%)$ \\
\hline Kochendorfer, $2012^{91}$ & $\begin{array}{l}\text { FL (4) } \\
\text { SMZL (1) }\end{array}$ & CD28 & $\begin{array}{l}60 \mathrm{mg} / \mathrm{kg} \mathrm{Cy} \times 2 \mathrm{~d} \\
25 \mathrm{mg} / \mathrm{m}^{2} \text { Flu } \times 5 \mathrm{~d}\end{array}$ & $5 / 5(100 \%)$ & $0 / 5(0 \%)$ \\
\hline Kochendorfer, $2015^{92}$ & $\begin{array}{l}\text { DLBCL (4), PMBCL (4), RT (1), } \\
\text { iNHL NOS (1), SMZL (1) }\end{array}$ & CD28 & $\begin{array}{l}\text { Cy } 120 \mathrm{mg} / \mathrm{kg} \\
\text { Flu } 25 \mathrm{mg} / \mathrm{m}^{2} \times 5 \mathrm{~d}\end{array}$ & $8 / 9(89 \%)$ & $5 / 9(56 \%)$ \\
\hline Wang, $2016 \dagger^{93}$ & $\begin{array}{l}\text { DLBCL (4) } \\
\text { MCL (4) }\end{array}$ & CD28 & Autograft conditioning & $2 / 2(100 \%) \ddagger$ & $2 / 2(100 \%) \ddagger$ \\
\hline Turtle, $2016^{86}$ & $\begin{array}{l}\text { DLBCL (11), TFL (11), FL (6), } \\
\text { MCL (4) }\end{array}$ & 41BB & Various (Cy, Flu) & $19 / 30(63 \%)$ & $10 / 30(33 \%)$ \\
\hline Locke, $2017^{94}$ & DLBCL (7) & CD28 & $\begin{array}{l}\text { Cy } 500 \mathrm{mg} / \mathrm{m}^{2}, \\
\text { Flu } 30 \mathrm{mg} / \mathrm{m}^{2} \text { x 3d }\end{array}$ & $5 / 7(71 \%)$ & $3 / 7(57 \%)$ \\
\hline Kochenderfer, $2017^{68}$ & $\begin{array}{l}\text { DLBCL (13), TFL (4), FL (2), } \\
\text { PMBL (2), MCL (1) }\end{array}$ & CD28 & $\begin{array}{l}\text { Cy } 300 \text { or } 500 \mathrm{mg} / \mathrm{m}^{2} \times 3 \mathrm{~d} \text {, } \\
\text { Flu } 30 \mathrm{mg} / \mathrm{m}^{2} \times 3 \mathrm{~d}\end{array}$ & $16 / 22(73 \%)$ & $12 / 22(55 \%)$ \\
\hline Neelapu, $2017^{11}$ & DLBCL (77), PMBCL or TFL (24) & CD28 & $\begin{array}{l}\text { Flu } 30 \mathrm{mg} / \mathrm{m}^{2} \text { for } 3 \mathrm{~d} \\
\text { Cy } 500 \mathrm{mg} / \mathrm{m}^{2} \text { for } 3 \mathrm{~d}\end{array}$ & $83 / 101(83 \%)$ & $55 / 101(55 \%)$ \\
\hline Schuster, $2017^{83}$ & DLBCL (14), FL (14) & 41BB & Various & $18 / 28(64 \%)$ & $16 / 28(57 \%)$ \\
\hline Schuster, $2018^{77} \S$ & DLBCL (93) & $41 \mathrm{BB}$ & $\begin{array}{l}\text { Flu } 25 \mathrm{mg} / \mathrm{m}^{2} \times 3 \mathrm{~d}, \\
\text { Cy } 250 \mathrm{mg} / \mathrm{m}^{2} \times 3 \mathrm{~d}, \\
\text { or Benda } 90 \mathrm{mg} / \mathrm{m}^{2} \times 2 \mathrm{~d}\end{array}$ & $48 / 93(52 \%)$ & $37 / 93(40 \%)$ \\
\hline Abramson, $2019^{78}$ & $\begin{array}{l}\text { DLBCL (137), TFL" (78), HGBCL } \\
\text { (36), PMBCL (15), FL3B (3) }\end{array}$ & $41 \mathrm{BB}$ & $\begin{array}{l}\text { Flu } 30 \mathrm{mg} / \mathrm{m}^{2} \times 3 \mathrm{~d} \\
\text { Cy } 300 \mathrm{mg} / \mathrm{m}^{2} \times 3 \mathrm{~d}\end{array}$ & $202 / 269(75 \%)$ & $143 / 269(53 \%)$ \\
\hline Wang, 2020 & MCL (60) & $\mathrm{CD} 28$ & $\begin{array}{l}\text { Flu } 30 \mathrm{mg} / \mathrm{m}^{2} \times 3 \mathrm{~d} \\
\text { Cy } 500 \mathrm{mg} / \mathrm{m}^{2} \times 3 \mathrm{~d}\end{array}$ & $56 / 60(93 \%)$ & $40 / 60(67 \%)$ \\
\hline
\end{tabular}

Benda, bendamustine; Cy, cyclophosphamide; DLBCL, diffuse large B-cell lymphoma; FL, follicular lymphoma; FL3B, follicular lymphoma grade 3B; Flu, fludarabine; HGBCL, high grade B-cell lymphoma; iNHL NOS, indolent B-NHL not otherwise specified; MCL, marginal zone lymphoma; PMBCL, primary mediastinal B-cell lymphoma; RT, Richter transformation; SLL, small lymphocytic lymphoma; SMZL, splenic marginal zone lymphoma; TFL, transformed follicular lymphoma; PMBCL

* Co-infused with a first-generation CAR

$\dagger$ only results for the $2^{\text {nd }}$ generation CAR included in this table

$\ddagger$ six participants were already in CR prior to autograft and CAR T-cell infusion

$\S 111$ participants treated but 93 with available data for efficacy

ๆ Includes patients with transformed from other indolent lymphomas as well as follicular lymphoma 


\subsection{Limitations in efficacy of current anti-CD19 CAR T-cells in r/r B-NHL}

It is clear that anti-CD19 CAR T-cell therapy has significantly improved the outcomes of patients with $\mathrm{r} / \mathrm{r}$ B-NHL in comparison to historical controls ${ }^{113676}$. However, at 12 months following treatment with current second generation autologous anti-CD19 CAR T-cell therapies, only $35-45 \%$ of patients remain progression free ${ }^{117677}$. In this section, some of the possible limitations to anti-CD19 CAR T-cell efficacy will be discussed.

\subsubsection{CAR T-cell persistence}

In patients with $\mathrm{r} / \mathrm{r}$ B-ALL, loss of anti-CD19 CAR T-cell persistence in the peripheral blood, appears to be the primary reason for CD19-positive disease relapse ${ }^{591}$. However, in patients with $\mathrm{r} / \mathrm{r}$ B-NHL, the importance of long term CAR T-cell persistence is less clear. In the ZUMA-1 Study, it was noted that $75 \%$ of patients with ongoing responses showed recovery of normal B-cells (a surrogate indicator for loss of anti-CD19 CAR T-cell persistence in the peripheral blood) at 9 months following treatment with axi-cel ${ }^{78}$. In conjunction with data from the NCI, which reported that three out of four patients with long term responses following anti-CD19 CAR T-cell treatment had B-cell recovery, these results are suggestive that long term remissions in patients with B-NHL can be achieved without ongoing CAR Tcell persistence in the peripheral blood ${ }^{92}$. Furthermore, recently published results from ZUMA-2 trial in patients with $\mathrm{r} / \mathrm{r}$ MCL, report that CAR T-cell persistence in the peripheral blood decreased over time in those with an ongoing response, suggesting that ongoing responses may not depend on long term CAR T-cell persistence ${ }^{87}$.

One possible hypothesis to explain this observation in patients with $\mathrm{r} / \mathrm{r} \mathrm{B}-\mathrm{NHL}$, is that a powerful CAR T-cell response could eliminate all malignant cells early after infusion, abrogating the need for ongoing CAR-cell persistence ${ }^{1}$. It is also possible that long term persistence of CAR T-cells at tumour sites is more important than persistence in the blood in maintaining disease responses in lymphoma ${ }^{93} 94$.

\subsubsection{Tumour Microenvironment}

The immuno-suppressive tumour microenvironment in solid tumours is a key factor that limits the efficacy of cancer immunotherapy ${ }^{95}$. In B-NHL, intra-tumoural T-cells often display abundant expression of the immune checkpoint protein Programmed cell death 
protein 1 (PD-1), and tumour cells frequently express Programme cell death-ligand 1 (PDL1); leading to inhibition of the anti-tumour function of effector T-cells within the tumour microenivronment ${ }^{96}$. Preclinical data has shown that upregulation of the PD-1/PD-L1 pathway directly inactivates CD28 signalling in CAR T-cells incorporating the CD28 costimulatory domain, thereby inhibiting CAR T-cell function ${ }^{97}$. Numerous preclinical studies in murine models have reported that PD-1 blockade can improve CAR T-cell activity and boost CAR T-cell expansion ${ }^{98} 99$. Clinical experience of combining immune checkpoint inhibitors with CAR T-cell therapy is limited, but prolonged CAR T-cell persistence and expansion has been reported with pembrolizumab (a PD-1 inhibitor) use, in patients with BALL and B-NHL 100101 . Therefore, the combination of immune check point inhibitors with anti-CD19 CAR T-cells in patients with $\mathrm{r} / \mathrm{r}$ B-NHL, may be a strategy to improve efficacy and clinical trials using this combination are now underway (Clinicaltrials.gov reference NCT02650999).

\subsubsection{T-cell dysfunction}

Patients undergoing T-cell harvest will typically have received multiple cytotoxic chemotherapy regimens and some may have received T-cell depleting treatments, hence there may be intrinsic T-cell defects at the time of harvest ${ }^{102}$. In the JULIET trial, there was failure to produce CAR T-cells in 12 out of 165 patients with DLBCL, suggesting that T-cell defects in these patients may impact CAR T-cell product manufacturing capabilty ${ }^{76}$. One study that investigated reasons for failure of CAR T-cell therapy in CLL patients, found that CAR Tcells in non-responders to treatment upregulated genes involved in effector differentiation, glycolysis, exhaustion and apoptosis ${ }^{103}$. In patients with DLBCL, a high percentage of $\mathrm{LAG}^{+} \mathrm{T}$-cells, a biomarker of T-cell exhaustion, was correlated with a lower response rate to anti-CD19 CAR T-cell therapy ${ }^{76}$. The impact of the condition of the CAR T-cell pool on the proliferation of CAR T-cells is still undetermined, but undoubtedly T-cell characteristics that impact expansion, persistence and tumour cytotoxicity, have a significant influence on treatment efficacy ${ }^{72}$.

\subsubsection{Immunologic rejection}

Most of the anti-CD19 CARs used in clinical practice contain scFv antibody regions derived from murine antibodies, namely FMC63, which are potentially immunogenic when 
administered to humans. While the murine derived scFV FMC63 has proven efficacy in multiple studies, repeated infusions have been shown to induce immunological anti-CAR responses $^{5} 104105$. Turtle et al reported that intensifying immunosuppressive chemotherapy prior to CAR T-cell infusion, improves CAR T-cell expansion at least partly due to reducing the anti-CAR immune response ${ }^{86}$. The use of anti-CD19 CARs with fully human variable regions is one potential way to reduce immunologic rejection of the CAR and improve clinical efficacy ${ }^{94}$.

\subsubsection{Predicting poor treatment outcomes}

Analysis of real world data in patients with $\mathrm{r} / \mathrm{r}$ DLBCL treated with axi-cel have shown that poor performance status (ECOG>1), high C-reactive protein (CRP) on the day of infusion, and high tumour bulk were associated with inferior outcomes ${ }^{106} 107$. Analysis of data from the ZUMA-1 trial reported by Locke et al demonstrated treatment benefit irrespective of the number of lines of prior treatment, however there was a suggestion that more heavily pretreatment had worse treatment outcomes ${ }^{108}$. There are now clinical trials in progress assessing anti-CD19 CAR T-cell therapy earlier on in the treatment of $r / r$ DLBCL, aiming to determine whether this strategy may benefit poor risk groups such as those with primary refractory disease following one line of treatment (eg ZUMA-7 trial and BELINDA trial, clinicaltrials.gov references NCT03391466 and NCT03570892). At the current time there are no validated pre-treatment clinical factors that are predictive of CAR T-cell efficacy ${ }^{106}$. Further collection of trial data, real world data from the use of axi-cel and tisagenlecleucel, plus longer duration follow-up; will hopefully help to identify patents with B-NHL most likely to benefit from anti-CD19 CAR T-cell treatment and help to improve treatment outcomes $^{106}$. 


\subsection{Anti-CD19 CAR T-cell therapy toxicities}

While anti-CD19 CAR T-cell trials have shown exciting results in terms of clinical efficacy, there are specific toxicities associated with CAR T-cell therapy which can be severe and potentially life threatening ${ }^{109}$. In particular, there are two key CAR T-cell related toxicities, cytokine release syndrome (CRS) and immune effector cell associated neurotoxicity syndrome (ICANS), which will be discussed in more detail within this section.

\subsubsection{Cytokine Release syndrome (CRS)}

CRS is a toxicity associated with many types of cellular immunotherapy including monoclonal antibodies, bispecific T-cell engager (BITE) therapy and immune checkpoint blockade $^{96110111}$. The term CRS is sometimes used interchangeably with the term 'cytokine storm;' but increasingly CRS has been defined as a distinct clinical syndrome with the term 'cytokine storm,' used more generally in cases of severe $\mathrm{CRS}^{111} 112$.

\subsubsection{Pathogenesis}

CRS is triggered when activation of T-cells via the TCR or CAR occurs on binding to their cognate antigen. This results in a release of pro-inflammatory cytokines from T-cells and other immune effector cells recruited to the tumour area ${ }^{113}$. It has become increasingly evident that proinflammatory cytokine released from activated CAR T-cells leading to the activation of macrophages and other myeloid cells, is a key driver in the development of $\mathrm{CRS}^{111114}$. For example, IFN- $\gamma$ can stimulate monocytes and other myeloid cells to secrete proinflammatory cytokines such as IL-12, IL-6, IL-1 $\beta$ and IL-15 and leads to low levels of anti-inflammatory cytokines including IL-4 and IL-10, driving the development of CRS ${ }^{115} 116$.

In a humanized mouse model with a high leukaemic burden, human monocytes were found to be the major source of IL-1 and IL- 6 during CRS, and CRS was prevented by depleting human monocytes or by blocking the IL-6 receptor with tocilizumab ${ }^{117}$. The interaction between macrophages and monocytes with T-cells helps to explain why macrophage activation syndrome can sometimes occur in patients treated with CAR T-cell therapy and why there is an overlap between macrophage activation syndrome and CRS ${ }^{118}$.

There is emerging evidence that vascular endothelial activation or dysfunction is contributory to pathophysiology of $\mathrm{CRS}^{113}$. Vascular endothelial cells have been found to be a key source 
of IL-6 during CRS ${ }^{119}$. One study which looked at 133 patients with r/r B-cell malignancies treated with anti-CD19 CAR T-cells, reported that markers of endothelial activation including Angiopoietin-2 and von Willebrand Factor were increased during severe CRS and also more elevated pre-lymphodepletion in patients who go on to develop severe CRS ${ }^{113}$. These findings are consistent with the presentation of vascular instability, capillary leak and consumptive coagulopathy, often seen in patients with severe $\mathrm{CRS}^{71}$.

\subsubsection{Clinical Features and grading systems}

CRS is characterised by fever, constitutional symptoms, tachycardia, hypotension, hypoxia, cardiac dysfunction and organ dysfunction ${ }^{11} 71768286$ 120-123. Constitutional symptoms include rigors, headaches, malaise, fatigue, arthralgia, nausea and vomiting; and organ dysfunction can comprise renal, hepatic and haematological toxicity including a coagulopathy ${ }^{109}{ }^{120}$. CRS usually occurs within the first week post CAR T-cell administration and usually peaks within 1-2 weeks post administration, although delayed cases of CRS are possible ${ }^{71} 109$.

Prior to 2019, there were a number of different grading systems used to report CRS in antiCD19 CAR T-cell trials including the Penn grading scale, the MD Anderson Cancer Centre/Lee grading scale and the CTCAE v4.0 scale 109120124125 . However, due to differences in the grading of CRS severity between different scales, comparison of CRS rates between different CAR T-cell trials is notoriously difficult ${ }^{124}$. For example, a patient requiring low dose vasopressors for hypotension would be classified as having grade 3 CRS by the Penn grading scale but only grade 2 CRS by the Lee scale ${ }^{120} 125$. In 2019, a consensus grading system for CRS was published by the American Society for Transplantation and Cellular Therapy (ASTCT) ${ }^{126}$. The ASTCT grading system for CRS solely uses clinical criteria based on the following parameters: Fever with temperature $\geq 38^{\circ} \mathrm{C}$, hypotension and hypoxia. This universal grading system for CRS should improve the ability to compare rates and severity of CRS across different trials and CAR T-cell products; providing an objective way to apply grading system for use in the post approval clinical setting as well as in future clinical trials $^{126}$.

\subsubsection{Frequency and severity}

Taking into account the limitations in comparing rates and severity of CRS between different clinical trials due to the different grading systems adopted, Table 2.2 compares the rates of 
CRS between the largest anti-CD19 CAR T-cell trials in r/r B-NHL. Severe CRS is usually reversible and with the appropriate management, the mortality secondary to CRS following anti-CD19 CAR T-cell therapy in r/r B-NHL, is thought to be less than $2 \%^{1171}{ }^{109}$. There are a number of possible reasons for the differences in the severe CRS rates observed across different trials including differing baseline patient, disease and CAR T-cell product characteristics; along with different CRS grading systems and management strategies in the early stages of CRS 113114124127 .

The impact of disease burden in the bone marrow on risk of developing severe CRS is well established in patients with B-ALL ${ }^{5}$. The impact of lymphoma disease burden on risk of severe CRS is less well established ${ }^{171}$, however updated analysis from the JULIET Study reports that increased lymphoma disease burden was associated with higher risk of any grade of CRS following treatment with tisagenlecleucel ${ }^{129}$. The impact of CAR T-cell dose and pharmacokinetics on CRS severity is discussed in Chapter 3. CAR T-cell therapies:

Optimising the dose.

Increasingly, predictive biomarkers for severe CRS, such as baseline thrombocytopenia and markers of endothelial activation such as angiopoietin-2 and von Willebrand factor, are being recognised 113130131 . It is hoped that utilising these predictive biomarkers, will lead to strategies to mitigate toxicity in those patients at the highest risk, so that CRS can be more effectively recognised and managed in the future ${ }^{71} 132-134$.

\subsubsection{Management}

There are no universal consensus guidelines for the management of CRS although several review articles outlining recommendations have been published ${ }^{71} 109120$. In the clinical trials setting some CAR T-cell products such as axi-cel were administered exclusively as an inpatient with a mandatory 7 day admission period, whereas other products such as tisagenlecleucel, were administered on both an inpatient and an outpatient basis 76109135136 . It is essential that CAR T-cell recipients receive appropriate education on CAR T-cell related toxicities (see Chapter 4.2.2 CAR T-cell toxicity education) ${ }^{137}$. 
One of the key immunosuppressive medications used in the treatment of CRS is the IL-6 receptor antagonist tocilizumab, which has been approved by the FDA for this indication ${ }^{138}$. As a condition for FDA approval of axi-cel and tisagenlecleucel, the product manufacturers are required to implement Risk Evaluation and Mitigation Strategies (REMS) at CAR T-cell treating centres, which includes ensuring there at least two doses of tocilizumab on site for each patient pre-treatment ${ }^{139}{ }^{140}$. Tocilizumab is universally recommended in different CRS management algorithms, although the recommended grade for administration, varies between guidelines $^{71} 109131$. Increasingly, there is expert consensus to adopt a lower threshold for tocilizumab and it is more commonly being used pre-emptively to mitigate the risks of severe $\mathrm{CRS}^{114141}$.

The use of corticosteroid therapy in CRS has typically been reserved for cases of CRS that are refractory to tocilizumab treatment ${ }^{109}{ }^{120}$. One reason for this is that corticosteroids are known to supress T-cell function and induce T-cell apoptosis; precluding the routine use of corticosteroids around the time of CAR T-cell treatment ${ }^{109} 142143$. However, the use of corticosteroids in the management of CRS, has not been found to objectively reduce antitumour responses ${ }^{134} 144145$. There is a theoretical concern that the use of tocilizumab may increase the risk of neurotoxicity, as the levels of IL-6 in the peripheral blood usually increase following tocilizumab administration, which may lead to passive diffusion of IL-6 into the cerebrospinal fluid $(\mathrm{CSF})^{109}$. Therefore, some experts within the field now advocate the use of short courses of corticosteroids in conjunction with tocilizumab for the management of lower grades of $\mathrm{CRS}^{146}$ (Cameron Turtle, personal communication, October, 2019). A summary of the CRS management algorithm adopted for the ENABLE Trial in Wellington Hospital (clinicaltrials.gov reference number NCT04049513) is shown in Appendix 1: Quicksheet for CRS management algorithm adopted for the ENABLE Trial in Wellington Hospital

In patients with severe CRS (grade $\geq 3$ ) that is not improving after three doses of tocilizumab and/or at least 24 hours of high dose corticosteroids, there may be a role for second-line agents, as well as continuing other standard of care measures detailed in local CAR T-cell toxicity guidelines. Three potential second line agents (anakinra, siltuximab and dasatanib) are currently recommended based on expert opinion, preclinical data and case reports ${ }^{117} 147$ ${ }^{148}$. There are no definitive recommendations on which second line agent is preferable. Anecdotally, anakinra (a human intereukin-1 receptor antagonist) has been used to treat CRS 
refractory to tocilizumab and corticosteroids in the US and in Australia (in patients treated with Yescarta ${ }^{\circledR}$ on the BELINDA trial, clinicaltrials.gov reference NCT03570892), and there is evidence for its efficacy in adult patients with Macrophage Activation Syndrome and Secondary Haemophagocytic Lymphohistiocytosis (MAS/HLH).

\subsubsection{Immune Effector Cell-associated Neurotoxicity syndrome (ICANS)}

Neurologic toxicities, previously referred to as CAR T-cell related encephalopathy syndrome (CRES) but recently renamed as Immune Effector Cell-Associated Neurotoxicity Syndrome (ICANS), are a frequent complication of CAR T-cell therapies ${ }^{120,149}$. ICANS typically presents as a toxic encephalopathy and can occur concurrently with CRS or in isolation following CAR T-cell therapy ${ }^{109}$. It can be a severe, life threatening toxicity following CAR T-cell therapy; but is usually reversible ${ }^{71}$.

\subsubsection{Pathogenesis}

The underlying pathogenesis of ICANS is yet to be fully determined ${ }^{109}$. However, there are a number of plausible mechanisms, with increasing supportive evidence ${ }^{114}$. Based on the finding that high serum levels of IL-6 and IL-15 have been observed patients with severe neurotoxicity, one postulated mechanism for ICANS is the passive diffusion of these cytokines into the $\mathrm{CNS}^{105109}$. This is supported by clear evidence that increased endothelial activation and microvascular permeability at the level of the blood brain barrier (BBB), plays a critical role in the development of neurotoxicity following CAR T-cell therapy ${ }^{130}{ }^{150}$. In an analysis of 53 patients with neurotoxicity following anti-CD19 CAR T-cell therapy, patients with severe neurotoxicity had earlier fevers with higher peak temperatures than those without severe neurotoxicity ${ }^{130}$. Furthermore these patients were found to have more severe hypoalbuminaemia and weight gain consistent with capillary leak syndrome, and much higher rates of disseminated intravascular coagulation (DIC); than those without severe neurotoxicity ${ }^{130}$. Higher peak levels of cytokines that activate endothelial cells such as IL-6, IFN $\gamma$ and TNF- $\alpha$, and other inflammatory markers such as CRP and serum ferritin; were correlated with the severity of neurotoxicity ${ }^{130}$. This is further supported by the observation that elevated levels of CSF protein, an indicator of BBB dysfunction, have been correlated with severe of neurotoxicity following CAR T-cell therapy ${ }^{150} 151$. 
A number of anti-CD19 CAR T-cell trials have reported that trafficking of CAR T-cells into the CNS, is often seen in patients with neurotoxicity ${ }^{105} 123152153$. However, other studies have found that CAR T-cell infiltration into the CSF does not correlate with the severity of neurotoxicity and that CAR T-cells can also be found in the CSF of patients without neurotoxicity, hence the impact of CAR T-cell trafficking into the CNS on neurotoxicity, is unclear $^{150} 154$. There is emerging evidence that high levels of cytokines, such as IFN $\gamma$, TNF- $\alpha$ and MCP-1, present in the CSF of patients with severe neurotoxicity, may trigger neurotoxicity independently of T-cells ${ }^{150}$. MCP-1, is secreted by macrophages, microglial cells and endothelial cells, and plays a key role recruiting monocytes and macrophages to the $\mathrm{CNS}^{155156}$. Norelli et al. demonstrated in a humanized mouse model, that monocyte derived IL-1 and IL-6 are required for the development of neurotoxicity, and inhibition of IL-1 specifically proved highly effective in protecting mice from lethal neurotoxicity ${ }^{117}$. Together these findings suggest that novel mechanisms for ICANS, whereby monocytes and macrophages are recruited to the CNS following endothelial damage and disruption to the $\mathrm{BBB}$, releasing IL-6 and IL-1; are key drivers for neurotoxicity.

\subsubsection{Clinical Features and grading systems}

ICANS typically presents as a toxic encephalopathy, initially with diminished attention, language disturbance and receptive aphasia, which progresses in some cases to disorientation and agitation. This can lead to seizures, mental obtundation and features of cerebral oedema in severe cases ${ }^{71}{ }^{109}$. Less commonly, other clinical features such as nerve palsies, focal sensory or motor defects, hallucinations and myoclonus have been observed ${ }^{82} 150157$. ICANS may occur concurrently with CRS, but can also present in patients without CRS or after CRS has resolved ${ }^{5123}$. Neelapu et al. reported that neurotoxicity following CAR T-cell therapy can be biphasic; the first phase typically occurring within the first five days following CAR Tcell infusion, and the second later after signs of CRS have abated ${ }^{109}$. In around $10 \%$ of patients, delayed onset neurotoxicity may occur in weeks 3-4 following anti-CD19 CAR Tcell treatment ${ }^{109158}$.

Early anti-CD19 CAR T-cell clinical trials predominantly used CTCAE for neurotoxicity grading $^{76} 8286$. In 2018, a multi-institutional group of oncologists leading anti-CD19 CAR Tcell trials across the US, published the CARTOX criteria for grading neurotoxicity ${ }^{109}$. The CARTOX grading incorporated a 10-point screening tool termed the CARTOX-10, 
analogous to the Mini Mental State Examination, but specifically designed to detect early features of neurotoxicity including language disturbance and handwriting impairment ${ }^{109}$. Other domains such as level of consciousness, motor symptoms, seizures and signs of elevated intracranial pressure (ICP) are also included in the CARTOX grading system. In 2019, an international consensus grading system for ICANS was published by the ASTCT ${ }^{126}$. The ASTCT grading system for ICANS, uses a 10-point score analogous to the CARTOX-10 score, but also includes an element for assessing receptive aphasia ${ }^{126}$. Other domains used in the ASTCT grading system have been simplified to make the system more clinically applicable than previous grading systems; for example by negating the requirement for CSF opening pressures and papilloedema grading, to be measured in order to determine cerebral oedema $^{126159}$.

\subsubsection{Frequency and severity}

The rates of neurotoxicity observed in the largest anti-CD19 CAR T-cell trials, is shown in Figure 2.2. ICANS can be severe and potentially life threatening, and fatal neurotoxicity typically due to cerebral oedema has been reported ${ }^{11,83,128,148}$. In one instance this led to termination of a trial (the ROCKET trial, Clinicaltrials.gov reference NCT02535364) 1183128 148160 . Overall, ICANS is usually reversible with a mortality rate thought to be under $3 \%{ }^{71} 130$. Most anti-CD19 CAR T-cell trials, including the pivotal ZUMA-1 and JULIET trials, excluded patients with CNS disease due to concerns about neurotoxicity ${ }^{11}{ }^{76}$. However, around 3\% of patients treated with Lisocabtagene Maraleucel on the TRANSCEND NHL 001 trial had secondary CNS lymphoma and there are increasing reports of commercial antiCD19 CAR T-cells being used in the real world setting to treat patients with secondary CNS lymphoma, without leading to severe neurotoxicity 77161162 .

As observed with CRS, the extent of disease burden in the bone marrow in patients with BALL, has been shown to be an independent risk factor for neurotoxicity ${ }^{5}{ }^{128}$. However, the impact of tumour bulk as a risk factor for neurotoxicity in patients with $\mathrm{r} / \mathrm{r}$ B-NHL is less clear. Real world data from the use of axi-cel in patients with $\mathrm{r} / \mathrm{r}$ B-NHL did not report an association between tumour bulk and neurotoxicity, whereas analysis from the TRANSCEND NHL 001 Study in patients with B-NHL, reported that high disease burden correlated with an increased risk of neurotoxicity ${ }^{107} 163$. In a retrospective review of 23 
patients with B-NHL and one patient with B-ALL that developed neurotoxicity following anti-CD19 CAR T-cell therapy, a low platelet count before CAR T-cell infusion was associated with more severe neurotoxicity and peak serum ferritin levels were significantly more elevated in patients with severe neurotoxicity, suggesting that ferritin may be a sensitive marker for following the clinical course of neurotoxicity ${ }^{164}$. In this study, high pretreatment LDH, a surrogate marker for disease burden in lymphoma patients, was a risk factor for a higher incidence of severe neurotoxicity, suggesting that a higher disease burden in B-NHL may lead to an increased risk of severe neurotoxicity ${ }^{164}$.

\subsubsection{Management}

No universal consensus guidelines for the management of ICANS exist although in common with CRS, several review articles outlining management recommendations for ICANS have been published ${ }^{71} 109{ }^{120}$. Patient education is crucial to ensure that patients with possible early symptoms of ICANS, contact their specialist centre so that appropriate interventions can be initiated $^{165}$. All clinical guidelines recommend close consultation with neurology specialists during the management of ICANS, which helps to guide the optimal use of CNS imaging and electroencephalopathy (EEG) monitoring in order to aid diagnosis and ICANS management. 71 109. Published guidance outlines the recommended use of tocilizumab and high dose corticosteroids in the management of ICANS. However, the optimal strategy for the management of patients with ICANS is still debated, with some treatment centres advocating early intervention with immunomodulators and others recommending a more conservative approach, with supportive care only in the earlier phases of ICANS 109130131136 . However, there is emerging evidence that early intervention in ICANS guided by the use of predictive biomarkers for neurotoxicity, is likely to improve patient outcomes 114164166 .

The evidence for the use of tocilizumab in the management of ICANS is debated. Some observational studies have reported that early treatment with tocilizumab was not associated with a decrease in the incidence or severity of neurotoxicity following anti-CD19 CAR T-cell therapy ${ }^{144} 167$. One possible reason for this finding is the poor CNS penetration observed with tocilizumab ${ }^{168}$. Furthermore, blockade of the IL-6 receptor with tocilizumab, may lead to increased levels of IL-6 in the circulation and potentially exacerbate neurotoxicity ${ }^{164} 169$. However, given the increased risk of severe neurotoxicity in patients with severe CRS, 
tocilizumab is usually administered to patients who develop neurotoxicity with concurrent CRS and some treatment centres advocate the use of tocilizumab as a first line therapy for neurotoxicity ${ }^{109130150166 .}$

The use of corticosteroids is an established treatment modality for ICANS and is adopted as first line therapy for neurotoxicity in a number of different guidelines 71109144 .

Dexamethasone has excellent CNS penetration and is widely used in the treatment of cerebral oedema due to other causes and hence it is commonly adopted in the management of ICANS to good effect ${ }^{109166170}$. Given that corticosteroids can supress T-cell function, there have been concerns that their use in ICANS management may decrease CAR T-cell efficacy ${ }^{142}$. However, this has not been seen in clinical practice, with increasing evidence that the use of corticosteroids to treat ICANS does not adversely impact CAR T-cell pharmacokinetics or clinical responses ${ }^{134} 145$. A summary of the ICANS management algorithm adopted for the ENABLE Trial in Wellington Hospital is shown in Appendix 2.

In patients with severe ICANS (grade $\geq 3$ ) that is not improving after the use of high dose corticosteroids and tocilizumab, there may be a role for using second-line agents. Siltuximab, a direct IL-6 antagonist has a rationale to treat ICANS by preventing the passive diffusion of IL-6 into the CNS and has been used in refractory cases of anti-CD19 CAR T-cell related neurotoxicity ${ }^{5171}$. There is a rationale for using anakinra in this setting; based on evidence from pre-clinical data studies suggesting IL-1 plays a crucial role in the pathogenesis of CAR T-cell related neurotoxicity and that anakinra was highly protective to prevent fatal neurotoxicity in a mouse model ${ }^{117}$.

Due to the high rates and potential severity of neurotoxicity following CAR T-cell therapy, prospective studies evaluating different treatment algorithms incorporating the use of biomarkers for neurotoxicity to guide management, are imperative in order to improve patient outcomes $^{164166}$. 
Table 2.2 CRS and Neurotoxicity incidence in trials of anti-CD19 CAR T-cells for R/R B-NHL

\begin{tabular}{|c|c|c|c|c|c|c|c|c|c|c|}
\hline \multirow[t]{2}{*}{ Study } & \multirow{2}{*}{$\begin{array}{l}\text { CAR co- } \\
\text { stimulatory } \\
\text { domain(s) }\end{array}$} & \multirow[t]{2}{*}{ Indications (n) } & \multirow{2}{*}{$\begin{array}{l}\text { CRS grading } \\
\text { system }\end{array}$} & \multicolumn{3}{|c|}{ CRS grade (rate, \%) } & \multirow{2}{*}{$\begin{array}{l}\text { Neurotoxicity } \\
\text { grading system }\end{array}$} & \multicolumn{3}{|c|}{ Neurotoxicity grade (rate, \%) } \\
\hline & & & & $1-2$ & $3-4$ & 5 & & $1-2$ & $3-4$ & 5 \\
\hline Neelapu $2017^{11}$ & $\begin{array}{l}\mathrm{CD} 28 \\
\mathrm{CD} 3 \zeta\end{array}$ & $\begin{array}{l}\text { DLBCL (77) } \\
\text { TFL or PMBCL (24) }\end{array}$ & Lee & 80 & 11 & $2^{*}$ & CTCAE & 37 & 28 & 0 \\
\hline Locke $2017^{94}$ & $\begin{array}{l}\text { CD28 } \\
\text { CD3 } \zeta\end{array}$ & DLBCL (7) & Lee & 71 & 14 & 0 & CTCAE & 29 & 57 & 0 \\
\hline $\begin{array}{l}\text { Kocbendorfer } \\
2017^{68}\end{array}$ & $\begin{array}{l}\text { CD28 } \\
\text { CD3 } \zeta\end{array}$ & $\begin{array}{l}\text { DLBCL (19) } \\
\text { FL (2) } \\
\text { MCL (1) }\end{array}$ & NR & 82 & 18 & 0 & CTCAE & 23 & 55 & 0 \\
\hline Schuster $2017^{83}$ & $\begin{array}{l}\text { 41BB } \\
\mathrm{CD} 3 \zeta\end{array}$ & $\begin{array}{l}\text { DLBCL (14) } \\
\text { FL (14) }\end{array}$ & UPenn & 39 & 18 & 0 & CTCAE & 29 & 11 & $4 \dagger$ \\
\hline $\begin{array}{l}\text { Turtle } 2016 \\
86\end{array}$ & $\begin{array}{l}41 \mathrm{BB} \\
\mathrm{CD} 3 \zeta\end{array}$ & $\begin{array}{l}\text { DLBCL (11) } \\
\text { TFL (11) } \\
\text { MCL (4) } \\
\text { FL (6) }\end{array}$ & CTCAE & 50 & 13 & 0 & CTCAE & $\mathrm{NR}$ & 25 & $3+$ \\
\hline Schuster $2018^{77}$ & $\begin{array}{l}41 \mathrm{BB} \\
\mathrm{CD} 3 \zeta\end{array}$ & DLBCL (111) & UPenn & 58 & 22 & 0 & CTCAE & 21 & 12 & 0 \\
\hline Abramson $2019^{78}$ & $\begin{array}{l}41 \mathrm{BB} \\
\mathrm{CD} 3 \zeta\end{array}$ & $\begin{array}{l}\text { DLBCL (137), TFL } \\
\text { (78), HGBCL (36), } \\
\text { PMBCL (15), FL3B } \\
\text { (3) }\end{array}$ & CTCAE & 42 & 2 & 0 & CTCAE & 30 & 10 & 0 \\
\hline Wang $2020^{87}$ & $\begin{array}{l}\text { CD28 } \\
\text { CD3 } \zeta\end{array}$ & MCL (68) & Lee & 91 & 15 & 0 & CTCAE & 63 & 31 & 0 \\
\hline
\end{tabular}

CARTOX, CAR-T-cell-therapy-associated TOXicity working group criteria ${ }^{176}$; Lee, Lee et al CRS revised grading system ${ }^{125}$; CTCAE, common terminology criteria for adverse events; DLBCL, diffuse large B-cell lymphoma; FL, follicular lymphoma; MCL, mantle cell lymphoma; NR, not reported; UPenn, University of Pennsylvania scale ${ }^{129}$; PMBCL, primary mediastinal B-cell lymphoma; TFL, transformed follicular lymphoma

* One patient died after developing haemophagocytic lymphohistiocytosis and one grade 5 cardiac arrest occurred in a patient with CRS

$\dagger$ One patient had progressive encephalopathy and death

† Fatal intracranial haemorrhage occurred in one patient 


\subsubsection{Other anti-CD19 CAR T-cell toxicities}

\subsubsection{Haemophagocytic Lymphohistiocytosis}

Haemophagocytic lymphohistiocytosis (HLH) is a clinical syndrome characterised by an overwhelming systemic inflammatory response with a cytokine release syndrome and multi organ dysfunction ${ }^{109}$. Many of the features of traditional HLH such as fevers, cytopenias, multi organ dysfunction, markedly elevated serum levels of ferritin, LDH, soluble CD25 and cytokines such as IL-6 and IFN $\gamma$ and hypofibrinogenemia may also be encountered in CRS, thereby making a distinction between these two syndromes difficult to make ${ }^{122}$. As such, there is considerable overlap between management of CRS and management of HLH post CAR T-cell therapy. There is no consensus recommendations for HLH following CAR T-cell therapy, but the use of tocilizumab and high dose corticosteroids is advocated, and other second line agents such as etoposide and anakinra, may have a role 109117172173 .

\subsubsection{B-cell depletion and hypogammaglobulinaemia}

CD19 is expressed by normal as well as malignant B-cells, and the depletion of normal polyclonal B-cell populations in recipients of anti-CD19 CAR T-cells is well documented ${ }^{1}$ 174. B-cell depletion could increase the risk of infections, including serious infections, and could potentially allow reactivation of latent viral infections such as hepatitis B virus (HBV), herpes zoster virus (HZV) or JC virus (the causative agent of progressive multifocal leukoencephalopathy, PML) ${ }^{175}$. While plasma cells lack CD19 expression, long-term persistence of anti-CD19 CAR T-cells may impede the generation of new plasma cells from $\mathrm{B}$ cells and prevent replenishment of the existing plasma cell population, leading to hypogammaglobulinaemia. In the ZUMA-1 study $31 \%$ of patients received intravenous immunoglobulin replacement for hypogammaglobulinaemia at some point following axi-cel infusion $^{78}$. Among recipients of tisagenlecleucel, $50 \%$ of patients achieved polyclonal B-cell recovery with a median time to onset of recovery of 6.7 months ${ }^{83}$.

\subsubsection{Cytopenias}

Prolonged cytopenias beyond 28 days following anti-CD19 CAR T-cell treatment, are reported in a proportion of patients. In the ZUMA-1 trial, 17\% of patients had grade 3 or higher cytopenias beyond 3 months post axi-cel infusion ${ }^{78}$. Fried et al. reported a biphasic 
nature of haematological toxicity following anti-CD19 CAR T-cell therapy, characterised by two trough levels with a temporary recovery in between, seen in $52 \%$ of patients with neutropenia and $34 \%$ of patients with thrombocytopenia ${ }^{176}$. While the first cytopenia trough appeared to correlate with CRS symptoms, the second trough occurred after the resolution of CRS in all cases, suggesting an alternative mechanisms for late onset cytopenias. One postulated mechanism, is that rapid recovery of early B-cells in the months following antiCD19 CAR T-cell therapy, leads to alterations of the levels of the chemokine SDF-1 in the bone marrow microenvironment, with lower levels of SDF-1 correlating with late onset neutropenia ${ }^{176}$.

Increasingly, granulocyte colony stimulating factor (GCSF), is used to treat prolonged neutropenia with reported efficacy and it is recommended in published guidance on CAR Tcell related toxicities ${ }^{109} 176$.

\subsubsection{Infections}

In addition to the direct immunosuppressive effects of anti-CD19 CAR T-cell therapy, the use of fludarabine and cyclophosphamide lymphodepleting chemotherapy can cause neutropenia and depletion of normal T-cell populations, particularly $\mathrm{CD}^{+} \mathrm{T}$-cells, adding to the risk of infection.

There may be an increased risk of viral infections and atypical organisms, such as pneumocystis jirovecii or mycobacteria. Prophylactic use of antivirals with activity against herpes zoster and simplex (such as valaciclovir) and of agents with activity against pneumocystis jirovecii (co-trimoxazole; pentamidine) are often administered as long term anti-microbial prophylaxis following anti-CD19 CAR T-cell therapy. Extended antimicrobial prophylaxis beyond one year may need to be considered in subjects with persistent severe CD4 lymphopenia ${ }^{177}$.

Reactivation of latent hepatitis B infection has been reported with anti-CD19 CAR T-cell therapies. In patients that test positive for the hepatitis B core antibody, the prophylactic use of appropriate antiviral agents for at least $12-18$ months is recommended ${ }^{175}$. 


\subsection{Selecting costimulatory domains for chimeric antigen receptors: functional and clinical considerations}

Following initial demonstrations that the CD28 domain within CARs was effective in providing costimulation, a range of other costimulatory domains have been assessed, including another Ig superfamily member, ICOS, and the TNFR superfamily members 41BB, OX40 and CD2 $27^{178} 179$. The use of costimulatory domains has since expanded from those derived from members of the Ig and TNFR superfamilies, to others signaling via cytoplasmic domains of IL-2Rb, IL-15R-a, CD40 or MyD88 ${ }^{180}$. The incorporation costimulatory molecules into CARs may be expected to confer varying degrees of costimulatory domain-specific activation, potentially with distinct impacts on CAR T-cell activity, proliferation and fate.

\subsubsection{CD28}

The Ig superfamily member CD28 is considered the prototypical T-cell costimulatory receptor and competes with its co-inhibitory receptor counterpart CTLA4 for binding to the B7 molecules CD80 and CD86 on Antigen Presenting Cells (APCs) ${ }^{181}$. While some costimulatory molecules are expressed only upon T-cell activation, CD28 is expressed by both resting and recently activated T-cells. The potency of unrestrained CD28 signaling is exemplified by the lethal lymphoproliferative syndrome occurring in CTLA4-deficient mice, and by the severe inflammatory syndrome seen in clinical trial recipients of an agonistic antiCD28 monoclonal antibody (mAb) that binds to the membrane-proximal loop of CD28 ${ }^{181}$. This CD28 super-agonist can trigger profound T-cell activation even in the absence of TCR ligation, overturning the long-standing concept that two signals are always required for T-cell $\operatorname{activation}^{181}$.

In vivo, 'second-generation' anti-CD19 CARs using CD28 costimulation demonstrate improved antitumor efficacy and persistence in comparison with first-generation CAR T-cells lacking a costimulatory domain ${ }^{6}$. Human $\mathrm{CD}^{+} \mathrm{CD} 28$-costimulated CAR T-cells have shown both central and effector memory phenotypes and exhibit rapid proliferation and IFN- $\gamma$

production in vitro upon recognition of target antigen ${ }^{182}{ }^{183}$. Recruitment of regulatory T-cells by CD28-costimulated CAR T-cell-derived IL-2 may limit antitumor activity in some 
models ${ }^{184}$. However, in general, $\mathrm{T}$ cells expressing second-generation CARs with CD28 costimulatory domains are associated with faster tumor elimination and activity at lower effector:target ratios compared to those expressing CARs with 4-1BB domains ${ }^{185}$. Consistent with animal studies, early-phase clinical trials suggest that CD28 costimulation augments CAR T-cell activity. Savoldo et $a l^{9}$ simultaneously administered autologous antiCD19 CAR T cells both without (first-generation) and with (second-generation) an intracellular CD28 costimulatory domain to six patients with r/r B-NHL without prior lymphodepleting chemotherapy. The CAR T cells lacking a CD28 costimulatory domain showed limited expansion and poor persistence, whereas the T cells expressing a CAR incorporating the CD28 domain underwent a greater degree of expansion and persisted for longer in all six patients9.

\subsubsection{4-1BB}

4-1BB (TNFRSF9, CD137) is an activation-inducedT- cell costimulatory molecule, first described in $1989^{186}$. A TNFR superfamily member, 4-1BB, is expressed on a subset of resting $\mathrm{CD}^{+} \mathrm{T}$ cells and is upregulated on both $\mathrm{CD}^{+}$and $\mathrm{CD} 8^{+} \mathrm{T}$ cells following activation $^{186}$.

T-cells expressing CARs that incorporate 4-1BB domains have been shown to express granzyme B, IFN-c, TNF-a, GM-CSF and the anti-apoptotic protein Bcl- $\mathrm{x}_{\mathrm{L}}{ }^{182}$. Incorporation of the 4-1BB transmembrane and cytoplasmic domain into a CAR leads to improved persistence and antitumor activity, and, compared to a CD28-costimulated CAR, prolonged T-cell division. However, CD28-based CARs provide more rapid and profound alterations in protein phosphorylation and greater effector T-cell activity ${ }^{187}$. In clinical trials, secondgeneration CARs incorporating a 4-1BB costimulatory domain appear to favor longer CAR T-cell persistence than those incorporating a CD28 domain ${ }^{11} 76185$.

Different malignancies might benefit from different CAR T-cell costimulatory domains: while the longer persistence of CAR T cells employing 4- 1BB costimulatory domains may be important for long-term remission of the precursor B-cell malignancy B-ALL, long-term CAR T-cell persistence may be less critical than early antitumor activity when treating mature B-NHL malignancies ${ }^{188}$. However, variability in CAR design, in vector, in 
manufacturing processes and between patient cohorts precludes definitive comparisons across CD28 and 4-1BB-based CAR T-cell clinical trials.

\subsubsection{Toll like receptors}

A number of Toll-like receptors (TLRs), as well as the TLR adaptor molecule MyD88, are expressed by activated T-cells, and TLRs can serve as costimulatory molecules within T cells, augmenting T-cell cytokine production and cytokine production in response to TCR stimulation $^{189}$. TLR2 stimulation of human T cells, for example, leads to enhanced Akt and Erk1/Erk2 phosphorylation in the presence of TCR stimulation ${ }^{189}$. The incorporation of a TLR2 endodomain into CD28-costimulated second-generation CARs enhanced CAR T-cell activity against CD19 and mesothelin-expressing tumors ${ }^{17} 18$.

\subsubsection{Third generation CARs - combining costimulatory domains}

As noted earlier, CAR T-cell activity could be enhanced by incorporating more than one costimulatory domain alongside $\mathrm{CD} 3 \zeta$, to produce a 'third-generation' CAR (see Figure 2.1). In vitro and xenograft studies indicate that T-cells expressing third-generation CARs can combine the tumoricidal capacity of CD28-based CARs with the persistence generated by 41BB- based CARs ${ }^{185}$. For example, Zhao et al. found that expression of a third-generation CAR construct combining a CD28 domain proximal to the membrane and a 4-1BB domain distally leads to increased T-cell expression of type 1 interferon pathway members, greater expansion of $\mathrm{CD}^{+}$and $\mathrm{CD}^{+} \mathrm{T}$ cells and improved B-ALL tumor regression in xenografts, compared to second-generation constructs ${ }^{185}$. Lower doses of CAR T cells expressing the third-generation construct were required for full antitumor activity, and the third-generation CAR T cells displayed longer persistence than their second-generation counterparts.

Clinical data also indicate that third-generation CARs lead to improved CAR T-cell expansion and persistence. In a phase I dose-escalation study, Ramos et al. simultaneously administered third-generation autologous anti-CD19 CAR T cells (incorporating both CD28 and 4-1BB costimulatory domains) and second-generation CAR T cells (expressing only the CD28 costimulatory domain) to patients with r/r B-NHL ${ }^{190}$. Six of 11 patients with active disease responded to treatment, including three complete responses. One case of severe CRS and one case of severe neurotoxicity was observed. In 10 out of 11 patients with active disease, greater expansion (up to 40-fold) of third-generation CAR T cells compared to 
second-generation CAR T cells was seen, and third-generation CAR T cells remained detectable at higher levels up to 160 days post-infusion. Moreover, only third-generation CAR T cells expanded significantly when infused to patients in remission after autologous stem cell transplantation, suggesting the third-generation CAR T cells can expand despite minimal CD19 antigen exposure ${ }^{190}$. Considering the results of Cheng et al., ${ }^{191}$ who reported no significant difference between two-second-generation (CD28 vs 4-1BB) CAR T cells coinfused in patients, Ramos et al. concluded that third-generation CAR T-cell therapy may be effective in the eradication of minimal residual disease and lead to longer, more durable remissions ${ }^{190}$.

Enblad et al. reported the outcomes of 15 patients with $\mathrm{r} / \mathrm{r}$ B-cell malignancies treated with autologous anti-CD19 CAR T cells incorporating both CD28 and 4-1BB costimulatory domains, four of whom did not receive lymphodepletion before CAR T-cell infusion ${ }^{192}$. Six achieved a CR, two of four with B-ALL and four of 11 with B-NHL. No treatment-related mortality was reported, and three cases of severe CRS or neurotoxicity were observed, comparable to the toxicity rates observed with second-generation CAR T-cell therapies ${ }^{109} 192$. Third-generation CAR T cells incorporating the Toll/interleukin-1 receptor (TIR) domain of Toll- like receptor 2 (TLR2), together with the CD3 $\zeta$ and CD28 intracellular domains, were administered to three patients with an extramedullary relapse of B-ALL. All three patients achieved a CR; final results of a phase I dose-escalation study using this construct are awaited $^{18}$.

Despite promising preclinical results and greater proliferative potential in early clinical trials, the clinical benefits of combining costimulatory domains within third-generation CAR T cells are yet to be conclusively demonstrated. In particular, the optimal dose of third-generation CAR T cell leading to improved clinical efficacy, without increased toxicity risk, is still unknown. A potential limitation of combining multiple costimulatory domains within a single CAR construct is that this might elicit tonic CAR signaling, leading to CAR T-cell exhaustion, paradoxically reducing activity. If this proves a limitation, providing multiple populations of second-generation CAR T cells, modifying second-generation CAR T cells to express an additional full-length costimulatory molecule in trans or providing inducible costimulatory signals might offer means to add costimulatory signals without inducing tonic signaling and exhaustion ${ }^{185} 191$. 
Ultimately, randomised clinical trials prospectively comparing CAR T cells incorporating various costimulatory domains are needed; such trials comparing second-generation CD28 and 4-1BB CAR T cells are underway and will shed light on the impact of costimulatory domain selection (ClinicalTrials.gov numbers NCT03191773 and NCT03076437, see Figure 2)

Figure 2.2 Anti-CD19 CAR T-Cell Trials by Costimulatory Domain

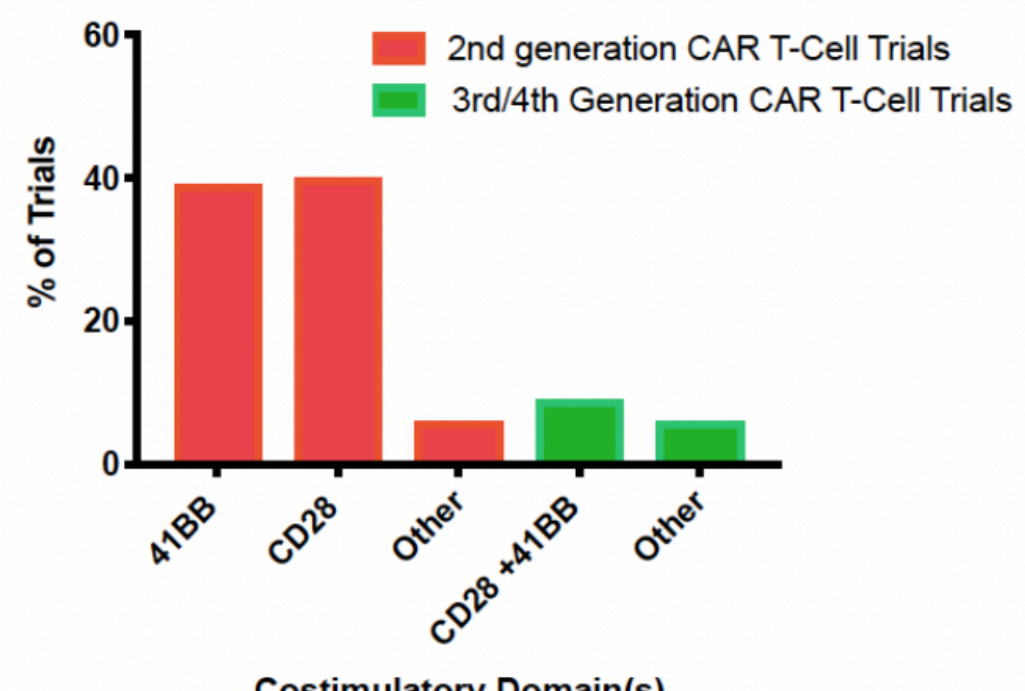

Survey of registered clinical trials utilising distinct costimulator domain structures. A search for anti-CD19 chimeric antigen receptor (CAR) T-cell trials registered at ClinicalTrials.gov obtained information on costimulatory domains in 55 trials. Information on costimulatory domains in a further 15 trials was obtained by emailing the trial contact on ClinicalTrials.gov, leading to a total of 69 anti-CD19 CAR T-cell trials available for data analysis. The second-generation CAR T-cell trials in 'other' include 2 trials where an admix of second-generation CARs containing $41 B B$ and CD28 was administered, and two trials where either $41 B B$ or CD28 containing second-generation CARs were administered. The third/fourth-generation CAR T-cell trials in 'other' includes one trial where an admix of a third-generation CAR utilising CD28 and $41 B B$ costimulation plus a CD28 containing second-generation CAR was administered, one trial where costimulation of CD28 and TLR2 was utilised, one trial where costimulation of CD28 and CD27 was utilised and one trial where costimulation of $41 B B$ and CD27 was utilised. 


\title{
3. CAR T-cell therapies: Optimising the dose
}

British Jounral of Clinical Pharmacology 2020 Mar 16. doi: 10.1111/bcp.14281

Dasyam N, George P, Weinkove R,

\begin{abstract}
T-lymphocytes can be genetically transduced to express a synthetic 'chimeric antigen receptor' (CAR) that re-directs their cytotoxic activity against a tumour-expressed antigen of choice. Autologous (patient-derived) CAR T-cells have been licensed to treat certain relapsed and refractory B-cell malignancies, and numerous CAR T-cell products are in clinical development. As living gene-modified cells, CAR T-cells exhibit unique pharmacokinetics, typically proliferating within the recipient during the first 14 days after administration before contracting in number, and sometimes exhibiting long-term persistence. The relationship between CAR T-cell dose and exposure is highly variable, and may be influenced by CAR design, patient immune function at the time of T-cell harvest, phenotype of the CAR T-cell product, disease burden, lymphodepleting chemotherapy, and subsequent immunomodulatory therapies. Recommended CAR T-cell doses are typically established for a specific product and indication, although for some products, stratification of dose based on disease burden may mitigate toxicity while maintaining efficacy. Re-evaluation of CAR T-cell dosing may be necessary following changes to the lymphodepleting regimen, for different disease indications, and, if product comparability cannot be demonstrated, after significant manufacturing changes. Dose escalation trials have typically employed ' $3+3$ ' designs, although this approach has limitations, and alternative phase I trial designs may facilitate the identification of CAR T-cell doses that strike an optimal balance of safety, efficacy and manufacturing feasibility.
\end{abstract}

\section{Keywords}

Chimeric antigen receptor therapy, pharmacokinetics, drug interactions, immunologic doseresponse relationship, drug toxicity, phase 1 clinical trials 


\subsection{Introduction}

Chimeric antigen receptor (CAR) T-cells are viable T-lymphocytes that have been genetically modified to express a synthetic receptor, which redirects T-cell cytotoxicity against a target of choice. Two CAR T-cell products, tisagenlecleucel (Kymriah ${ }^{\circledR}$, Novartis) and axicabtagene ciloleucel (Yescarta ${ }^{\circledR}$, Gilead), both targeting the B-cell antigen CD19, have been licensed for treatment of $\mathrm{r} / \mathrm{r}$ B-cell malignancies ${ }^{193}$, and many other CAR T-cell products are in clinical development ${ }^{194}$.

Chimeric antigen receptors have a modular design, permitting countless variations of specificity and function. Considerable variation between CAR T-cell products can also arise from the phenotype of T-cells used for CAR T-cell manufacture, the method of gene modification, promoters and other features of the transgene that affect CAR expression, methods of T-cell stimulation, expansion and culture, and formulation and delivery of the final product.

Upon intravenous administration to recipients, CAR T-cells may become activated through binding to the CAR target on tumour (or on normal) cells, and proliferate to a variable degree. Following a period of activation and expansion, CAR T-cells become exhausted, and contract in number. A population of CAR T-cells may persist long-term within the recipient, with the potential to mediate both durable anti-tumour efficacy and toxicity.

This review provides a brief background to CAR T-cell therapies, discusses the principal methods used to quantify CAR T-cells, summarises current knowledge of CAR T-cell pharmacokinetics, and lists factors known to influence CAR T-cell pharmacokinetics and pharmacodynamics. Key considerations for the design and interpretation of CAR T-cell dose escalation trials, and for selection of an optimal dose, will be discussed.

\subsection{Background}

\subsubsection{History of CAR T-cells}

The use of autologous T-lymphocytes as a cancer therapy is not new, with reports of dramatic clinical responses among some recipients of 'lymphokine-activated killer' cells and expanded tumour-infiltrating lymphocytes since the $1980 \mathrm{~s}^{52} 195196$. However, most do not respond to 
these adoptive T-cell therapies, and it is possible that many individuals with cancer lack a Tcell population capable of recognising and eradicating their tumour.

T-cells can be redirected to target an antigen of choice by introducing a gene encoding a synthetic receptor, overcoming reliance on the endogenous T-cell receptor (TCR) repertoire. CARs combine elements of an antibody (the B-cell receptor) to bind to an antigen, and a component of the TCR complex to signal to the T-cell ${ }^{3197}$.

So-called 'first-generation' CARs combined an extracellular antigen recognition domain (single chain variable fragment, $\mathrm{scFv}$ ) derived from a monoclonal antibody linked via a hinge and transmembrane domain to the intracellular CD3 $\zeta$ region of the TCR (Figure 2.1) ${ }^{2}$. While first generation CARs led to T-cell activation in upon binding to the target of the scFv, the Tcells exhibited only limited proliferation and function ${ }^{6}$.

'Second-generation' CARs add an intracellular co-stimulatory domain, often derived from the T-cell co-stimulatory molecules CD28 or 4-1BB, and result in more robust T-cell proliferation and function in response to CAR binding ${ }^{178} 198199$.The licensed CAR T-cell therapies tisagenlecleucel and axicabtagene ciloleucel both employ 'second-generation' CAR constructs, incorporating co-stimulatory domains from 4-1BB and CD28, respectively (Figure 2.1).

A myriad of other CARs have been produced, including CARs containing alternative costimulatory domains, and 'third-generation' CARs combining two intracellular costimulatory domains (Figure 2.1) 200 $^{2}$. In addition, 'bicistronic' transgenes can be introduced into T-cells to drive expression of a second protein alongside the CAR, such as the T-cellstimulating cytokine IL-12, or a protein that facilitates CAR T-cell detection or depletion ${ }^{201}$.

\subsubsection{Manufacture of CAR T-cells}

A typical manufacturing process for CAR T-cells is summarised in Figure 3.1. Patient or donor leukocytes are obtained by leukapheresis, from which T-cells are isolated. T-cells are activated and genetically transduced with a CAR-encoding transgene in vitro. Transduction can be achieved using vectors derived from human retro- or lentiviruses (incorporating safety modifications to limit virulence and prevent viral replication). An alternative is genetic 
transduction using a transposon/transposase system, such as "Sleeping beauty" or PiggyBac $^{202}$.

Following transduction, T-cells are expanded in vitro in the presence of specific cytokines. Various in-process controls are applied, and following manufacture, each CAR T-cell product undergoes product release testing. Product release criteria typically include tests to ensure viability, identity, purity, microbiological sterility and stability ${ }^{203}$. In addition to product release criteria, additional tests are often conducted to assess the proportion of various T-cell subsets within the product, and to characterise CAR T-cell function, or potency ${ }^{204}$.

Figure 3.1 Manufacture of chimeric antigen receptor T-cells
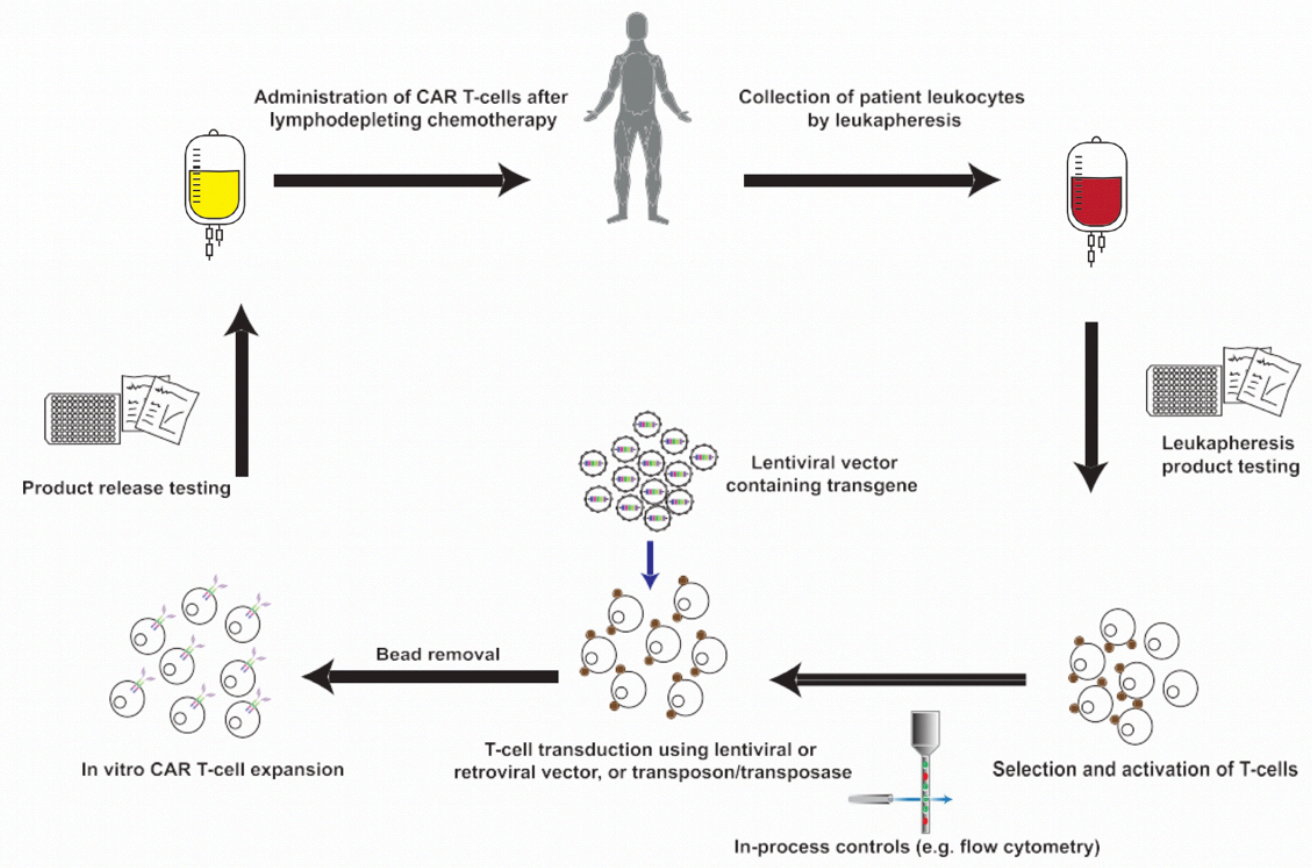

Manufacture of autologous CAR T-cells typically begins with patient blood leucocytes obtained by leukapheresis. A sample of this input product is sent for screening tests, while Tcells are purified ahd activated using immunomagnetic beads or plate-bound antibodies. Inprocess controls may include purity checks by flow cytometry. A transgene is introduced using a lentiviral or retroviral vector, or a transposon/transposase system. CAR T-cells are expanded in vitro using optimised cell culture conditions and in the presence of cytokines. Product release testing is required before CAR T-cells are released for administration to the patient, typically following lymphodepleting chemotherapy 


\subsection{Quantifying CAR T-cells}

CAR T-cell pharmacokinetics are most commonly determined by quantitative polymerase chain reaction (qPCR) for the transgene, or by direct enumeration of CAR-expressing T-cells using flow cytometry, using peripheral blood or plasma samples.

\subsubsection{Quantitative Polymerase Chain Reaction}

The number of CAR T-cells can be estimated by qPCR for the CAR-encoding transgene. A transgene-encoding plasmid is serially diluted to generate a standard curve. After appropriate corrections for qPCR reaction efficiency and input DNA quantity, the number of transgene copies per unit DNA can be determined within CAR T-cell recipient whole blood ${ }^{205} 206$ or peripheral blood mononuclear cells (PBMCs) ${ }^{207}$.

Advantages of quantifying CAR T-cells by qPCR are that it is a sensitive method, can be applied to stored DNA samples, and will detect CAR DNA even if the CAR has been downregulated on the T-cell surface. Disadvantages are that qPCR can be time consuming, limiting applicability for 'real-time' analysis, and that it does not distinguish between cells that express the CAR and those that harbour one or more CAR transgene copies but are incapable of expressing the receptor.

\subsubsection{Flow cytometry}

Flow cytometry is routinely used in clinical laboratories to quantify lymphocyte subsets in blood. A cell suspension is labelled with fluorescent reagents (often monoclonal antibodies), then analysed to determine fluorescence characteristics on a 'per cell' basis. Several reagents can be used to detect surface expression of CARs, including monoclonal antibodies specific for the $\mathrm{scFv}$ domain of the $\mathrm{CAR}^{208}$, protein $\mathrm{L}^{209}$, or the target antigen itself ${ }^{210}$. For some products, CAR T-cells can be enumerated by detecting a second protein encoded alongside the CAR, such as a truncated EGFR polypeptide ${ }^{211}$.

Advantages of flow cytometry over qPCR include detection only of T-cells expressing the CAR protein, and the potential to simultaneously assess expression of other proteins on a percell basis. This allows, for example, the separate enumeration of $\mathrm{CD}^{+}$and $\mathrm{CD} 8^{+} \mathrm{CAR} \mathrm{T}-$ cells, or the determination of memory phenotype of circulating CAR T-cells. Disadvantages of flow cytometry include a lack of assay standardisation between laboratories, difficulty 
distinguishing low-level CAR expression from background fluorescence, the need for intact cells to perform the assay, and in some instances, a paucity of available reagents for CAR detection. Compared to qPCR, flow cytometry is relatively insensitive, limiting its utility for the detection of low-level CAR T-cell persistence. For example, Maude et al. reported the detection of persisting CAR T-cells by qPCR, but not by flow cytometry, up to two years after therapy ${ }^{123}$.

\subsubsection{Imaging CAR T-cells}

A general limitation of both flow cytometry and qPCR is that they are typically applied to liquid samples, so do not fully reflect CAR T-cell tissue distribution. CAR T-cells are expected to traffic to, and potentially proliferate and persist within, tissue and tumour locations. While tissue biopsies can provide proof of principal of CAR T-cell tissue infiltration ${ }^{212}$, invasive tests are unsuitable for serial monitoring.

The direct labelling of CAR T-cells with isotopes or superparamagnetic particles can enable their localisation using $\gamma$-camera, positron emission tomography (PET) or magnetic resonance imaging 207213214 . However, the requirement for a relatively large number of labelled cells in close proximity to detect a signal, dilution of the label during CAR T-cell proliferation, and loss of the label during CAR T-cell death, can all limit the sensitivity and specificity of imaging, especially beyond the first few days after CAR T-cell administration. An alternative approach is to administer a PET tracer that detects a second protein encoded by the transgene. This can enable, for example, PET imaging of a truncated PSMA expressed on CAR T-cells, or of viral thymidine kinase activity within CAR T-cells ${ }^{215} 216$. These techniques are not used commonly, but can inform CAR T-cell pharmacokinetics in research settings.

\subsection{CAR T-cell pharmacokinetics}

Unlike conventional drugs, CAR T-cells can proliferate within the recipient, resulting in a highly variable relationship between dose and exposure. CAR T-cells are usually administered intravenously, and following initial localisation to the lung, may redistribute to the spleen and bone marrow within hours 207213217 . It is assumed that, like normal T-cells, 
CAR T-cells are capable of distributing widely into other tissues, including to tumour sites, but pharmacokinetic data are usually limited to circulating CAR T-cell numbers.

In clinical experience of second-generation anti-CD19 CAR T-cell therapies, intravenous administration of CAR T-cells is followed by a brief decline in circulating levels, possibly reflecting distribution to tissue sites, followed by a rapid increase in numbers following activation and proliferation of the CAR T-cells ${ }^{154}$. Circulating CAR T-cell numbers frequently peak within two weeks of administration, after which they decline in number at a variable rate (Figure 3$)^{133}$.

The maximum CAR T-cell level $\left(\mathrm{C}_{\max }\right)$ and area under the concentration-time curve between the time of CAR T-cell administration and day $28\left(\mathrm{AUC}_{0-28 \mathrm{~d}}\right)$ are frequently used as measures of early CAR T-cell exposure (see Figure 3.2) ${ }^{154}$. Both reflect early CAR T-cell expansion, and are associated with clinical response to therapy, with higher $\mathrm{AUC}_{0-28 \mathrm{~d}}$ values among those who respond to second-generation anti-CD19 CAR T-cell products, than among nonresponders ${ }^{11} 154$.

In some recipients, CAR T-cells persist long-term, as determined by qPCR or, for anti-CD19 CAR T-cell products, as inferred by persistent depletion of normal B-cells (B-cell aplasia; an on-target, off-tumour toxicity). CAR T-cells can persist longer than 4 years after therapy ${ }^{125}$, although persistence rates are variable, and may depend upon the CAR target, co-stimulatory domains within the CAR, CAR T-cell phenotype and patient and disease characteristics ${ }^{133} 218$. 
Figure 3.2 CAR T-cell pharmacokinetics

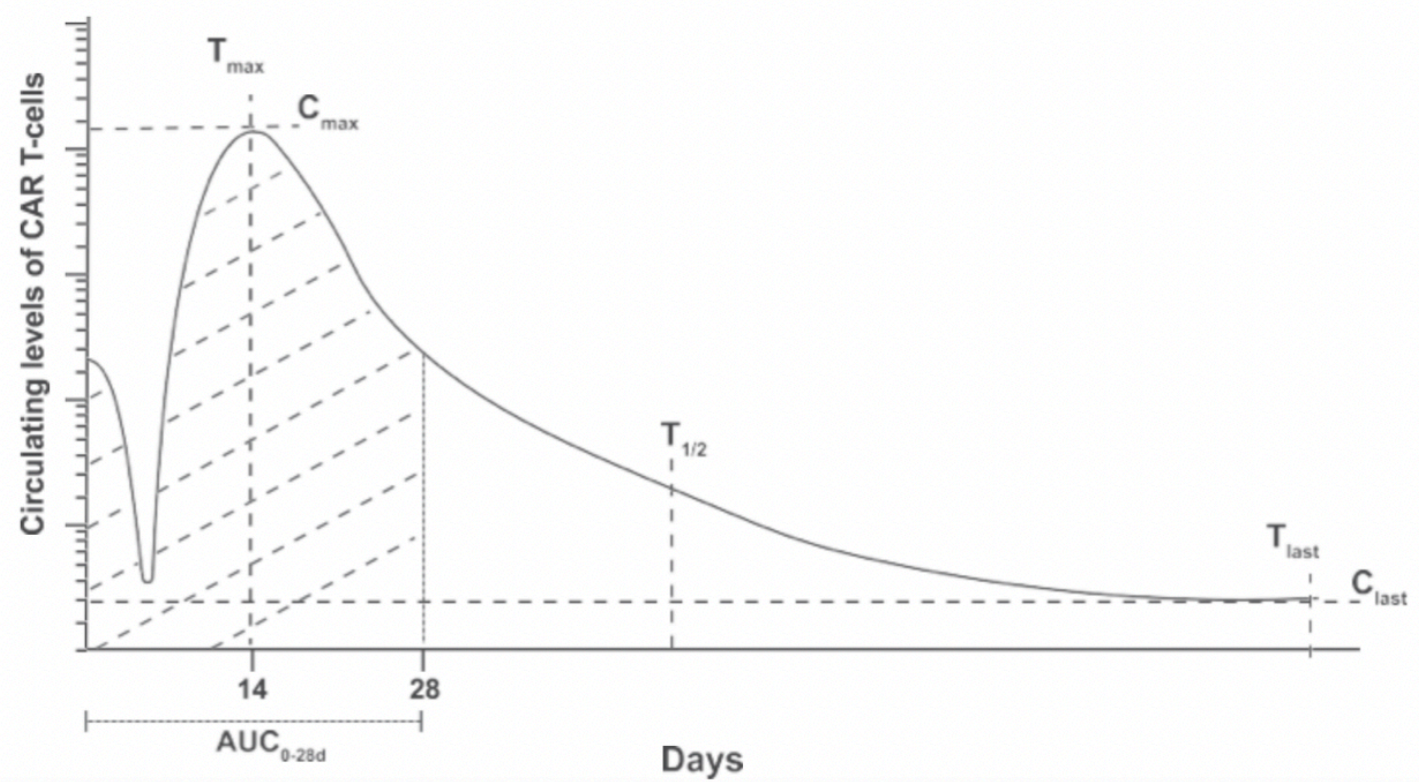

For second-generation anti-CD19 CAR T-cells for B-cell malignancies, an initial drop in CAR T-cell levels after infusion is followed by a period of CAR T-cell expansion.

Peak levels $\left(C_{m a x}\right)$, typically occur within 14 days $\left(t_{\max }\right) ; t_{1 / 2}$ represents the half-life, $t_{\text {last }}$ represents the time at which the last measurable level $\left(C_{\text {last }}\right)$ was recorded. $A U C_{0-28 d}$ represents the area under the concentration-time curve during the first 28 days after CAR Tcell administration. The y-axis represents a log-scale in terms of differences in the CAR Tcell concentrations

\subsubsection{Factors influencing pharmacokinetics}

\subsubsection{Lymphodepleting chemotherapy}

Treatments that deplete circulating lymphocytes increase systemic availability of T-cellsimulating cytokines such as IL-7 and IL-15 82219220 , and have long been employed to augment expansion of subsequently-administered T-cells ${ }^{221}$. Lymphodepletion before administration of CAR T-cells results in improved CAR T-cell expansion, persistence, and anti-tumour activity ${ }^{66222}$. Lymphodepleting chemotherapies are widely employed before CAR T-cell administration within clinical trials and in routine practice ${ }^{1223}$, reported regimens including cyclophosphamide with fludarabine, cyclophosphamide alone and bendamustine ${ }^{511}$ 125136224 . 
While the dramatic effect of lymphodepleting chemotherapy on early CAR T-cell

pharmacokinetics is principally attributed to increased levels of cytokines ${ }^{65225}$, lymphodepletion may also facilitate long-term CAR T-cell persistence: lymphodepleting chemotherapy can prevent immunologic rejection of CAR T-cells due to immune responses against murine-derived or human epitopes of the $\mathrm{CAR}^{65} 86$.

As CAR T-cell pharmacokinetics are strongly influenced by the selection and dose of lymphodepleting chemotherapy administered beforehand, package inserts for both tisagenlecleucel and axicabtagene ciloleucel recommend specific cyclophosphamide and fludarabine dosing schedules ${ }^{226} 227$.

\subsubsection{Tumour Burden}

Although supported by serum cytokines, early expansion of CAR T-cells in vivo is driven by CAR binding to tumour (and normal) cells that express the CAR target. Among patients with B-ALL the presence of greater than 5\% leukaemic blasts in the bone marrow was associated with higher $\mathrm{C}_{\max }$ and higher CAR T-cell levels 28 days after CAR T-cell infusion ${ }^{5}$. Similarly, in B-NHL, expansion of both second- and third-generation CAR T-cells was greater in patients treated at the time of active lymphoma, than among those treated following autologous stem cell transplantation, in the setting of minimal tumour burden ${ }^{190}$.

\subsubsection{CAR Target and SCFV}

While the ideal CAR target might be exclusively and uniformly expressed at a high level on tumour cells, yet not expressed on normal cells ${ }^{228}$, the targets most successfully employed for CAR T-cell therapy of B-cell cancers do not meet these criteria: CD19, CD22 and B-cell maturation antigen (BCMA) are all expressed on normal as well as malignant cells. Indeed, exposure to target antigen on normal cells may contribute to CAR T-cell expansion and persistence or, conversely, CAR T-cell exhaustion ${ }^{229}$. Fortunately, both B-cell aplasia and hypogammaglobulinaemia (reduction of normal immunoglobulin due to a depletion of plasma cells) are manageable on-target off-tumour toxicities.

For a given antigenic target, the scFv domain, which determines CAR binding affinity and specificity, can be expected to influence CAR T-cell pharmacokinetics and pharmacodynamics ${ }^{230}$. A higher rate of severe neurotoxicity was observed in recipients of an 
anti-CD19 CAR T-cell therapy incorporating an scFv from the $\mathrm{SJ} 25 \mathrm{C} 1$ antibody clone than those who received a product using the scFv from the FMC63 clone, although the relative contribution of the $\mathrm{scFv}$ compared to other factors remains uncertain ${ }^{11} 130$.

A particular challenge is identification of suitable targets for CAR T-cell therapy of solid cancers, for which complete response rates are very low ${ }^{231}$. Identification of uniformlyexpressed solid tumour antigens capable of supporting robust CAR T-cell activation, expansion and on-target cytotoxicity remains an issue, alongside measures to overcome tumour-related immunosuppression and to assure CAR T-cell infiltration into the tumour ${ }^{232}$.

\subsubsection{Co-stimulatory domains}

The most frequently employed co-stimulatory domains within CARs are CD28 and 4-1BB ${ }^{200}$. The CD28 domain is associated with rapid CAR T-cell expansion and an effector memory phenotype 200233 , while the 4-1BB co-stimulatory domain is associated with slower initial CAR T-cell expansion, enhanced persistence and a central memory phenotype ${ }^{200233}$. Many other co-stimulatory domains have been reported, and their selection may influence CAR Tcell pharmacokinetics and pharmacodynamics ${ }^{200}$.

Third-generation CAR T-cells employ two co-stimulatory domains, and are associated with greater CAR T-cell expansion and persistence than second-generation counterparts both preclinically, and in a small clinical trial ${ }^{234}$. Whether this will translate to improved clinical outcomes is not yet clear.

\subsubsection{CAR T-cell phenotype}

Based on the expression of specific surface markers, normal T-cells can be divided into various subsets. These include $\mathrm{CD}^{+}$and $\mathrm{CD} 8^{+} \mathrm{T}$-cells, and various 'memory' and more highly-differentiated 'effector' T-cell subsets.

While most CAR T-cell products can be expected to contain a variable mixture of $\mathrm{CD}^{+}$and $\mathrm{CD}^{+} \mathrm{T}$-cells, some advocate for CAR T-cell products of defined CD4:CD8 ratio, citing improved efficacy and toxicity ${ }^{235}$, albeit at the expense of a more involved manufacturing process $^{236}$. 
In retrospective analyses, CAR T-cell products bearing a greater fraction of cells with memory phenotypes, or expressing higher levels of genes associated with T-cell memory, are associated with improved expansion and persistence ${ }^{103237}$. Some manufacturers select memory T-cell subsets, or modify culture conditions during in vitro CAR T-cell expansion, to raise the fraction of CAR T-cells expressing memory markers ${ }^{238-242}$.

\subsubsection{Drug interactions}

As a cellular product, CAR T-cells are not expected to exhibit typical pharmacokinetic interactions. However, the significant impact of prior lymphodepleting chemotherapy on CAR T-cell pharmacokinetics has already been discussed, and subsequent immunomodulatory treatments, including those manage CAR T-cell toxicities, could affect CAR T-cell expansion, persistence or function.

\subsubsection{Anti-IL-6 therapy}

CRS and ICANS are among the most significant CAR T-cell-related toxicities ${ }^{109}$. The pathogenesis of CRS in particular appears to involve the activation of myeloid cells by CAR T-cell-derived IL- ${ }^{117}$. Anti-IL-6 therapy with the monoclonal antibodies tocilizumab or siltuximab, can lead to rapid clinical improvement ${ }^{109126}$.

While pre-clinical studies suggest IL-6 is not directly involved in CAR T-cell lysis of tumour cells ${ }^{116}$, an adverse impact of IL-6 blockade on CAR T-cell pharmacokinetics or pharmacodynamics is a theoretical concern. Reassuringly, clinical experience suggests that clinical response rates are not adversely affected by tocilizumab administration ${ }^{11}{ }^{136}$. For example, in a retrospective analysis of children receiving anti-CD19 CAR T-cells for B-ALL, early tocilizumab use did not appear to affect $\mathrm{C}_{\max }, \mathrm{AUC}_{0-28 \mathrm{~d}}, \mathrm{CAR} \mathrm{T}$-cell persistence, or $\mathrm{CR}$ rate $^{144}$. Similarly, a retrospective analysis of a cohort of adults with B-NHL reported that tocilizumab had no effect on CAR T-cell $\mathrm{C}_{\max }{ }^{167}$. Pending the results of prospective trials, these observations can be considered encouraging, and concerns about CAR T-cell pharmacokinetics or function should not deter the use of anti-IL-6 therapies to manage toxicities. 


\subsubsection{Corticosteroids}

High-dose corticosteroids induce T-cell apoptosis ${ }^{243}$, and are a recommended treatment for refractory CRS and severe ICANS ${ }^{126}$.

Retrospective studies have not associated corticosteroid administration with a significant reduction in $\mathrm{CAR} \mathrm{T}$-cell $\mathrm{C}_{\max }, \mathrm{AUC}_{0-28 \mathrm{~d}}$ or clinical response rates ${ }^{11}{ }^{144}$. However, corticosteroid use was restricted to patients with severe tocilizumab-refractory CRS or ICANS, among whom brisk CAR T-cell expansion may have already been well-established ${ }^{71}$ ${ }^{126}$, and an impact of corticosteroids on early CAR T-cell expansion, or on persistence or durability of anti-tumour responses, is not excluded ${ }^{71}$. Accordingly, many clinical protocols restrict corticosteroid use after CAR T-cell therapies to specific settings, in which the benefits are likely to outweigh the risks.

\subsubsection{Checkpoint blockade}

Like normal T-cells, CAR T-cells are thought to become 'exhausted' following prolonged exposure to antigen, characteristically upregulating expression of the inhibitor programme cell death-1 (PD-1) receptor ${ }^{99}$.

The function of normal anti-tumour T-cells can be enhanced by therapies that prevent PD-1 binding to its receptor, providing rationale for combining antibodies that block PD-1, such as pembrolizumab or nivolumab, with CAR T-cell therapies ${ }^{232}$. In preclinical studies, PD-1 blockade enhances CAR T-cell expansion and function ${ }^{98} 99$, and anecdotally, delayed rises in CAR T-cell levels and clinical responses have been observed among patients receiving antiPD1 therapies for progressive B-NHL and B-ALL following disease progression after antiCD19 CAR T therapy ${ }^{244-246 .}$

\subsection{Optimising CAR T-cell dose}

\subsubsection{Inter-individual variability}

The licensed, and the majority of investigational, CAR T-cell therapies are manufactured from autologous (patient-derived) T-cells. As such, a CAR T-cell product will be influenced by a patient's immunological status before leukapheresis ${ }^{247}$. Patient factors, such as age, underlying disease and the number of circulating T-lymphocytes influence the probability of successful CAR T-cell manufacture ${ }^{248}$. Moreover, even when CAR T-cell products are 
successfully manufactured and meet product release criteria, characteristics of the starting Tcells may influence subsequent CAR T-cell pharmacokinetics and pharmacodynamics. For example, a higher frequency of a memory subset of $\mathrm{CD}^{+} \mathrm{T}$-cells within leukapheresis product was associated with clinical response to subsequent anti-CD19 CAR T-cell therapy for chronic lymphocytic leukemia ${ }^{103}$. Additionally, pre-clinical data suggest that interindividual differences in the immunological milieu at the time of CAR T-cell administration can influence CAR T-cell pharmacokinetics ${ }^{249-251}$. Therefore, while seeking to optimise CAR T-cell dosing, it must be acknowledged that inter-individual variation in CAR T-cell pharmacokinetics may be considerable, and that the factors governing this variation are still being explored

\subsubsection{Dose-exposure and dose-response relationship}

CAR T-cell doses are frequently provided as the number of viable CAR-expressing T-cells per kilogram recipient body weight, although some studies report CAR T-cells per $\mathrm{m}^{2}$ recipient body surface area, or total dose administered. Because CAR T-cells proliferate within the recipient, a linear relationship between CAR T-cell dose and exposure cannot be assumed. Moreover, because CAR T-cells are typically delivered as a single infusion, there is little opportunity to adjust exposure once the therapy has been administered.

Among patients with $\mathrm{r} / \mathrm{r}$ B-ALL receiving second-generation anti-CD19 CAR T-cells after fludarabine and cyclophosphamide lymphodepletion, a dose of $2 \times 10^{5} \mathrm{CAR}$ T-cells kg-1 was associated with later attainment of a lower $C_{\max }$ than a tenfold higher dose ${ }^{86}$. Similarly, among individuals with $\mathrm{r} / \mathrm{r}$ myeloma receiving second-generation anti-BCMA CAR T-cells, minimal or no CAR T-cell expansion was observed at the two lowest dose levels, while robust expansion was observed after higher doses ${ }^{252}$. A dose-exposure relationship has also been reported for third-generation anti-CD19 CAR T-cells: $\mathrm{C}_{\max }$ was lower among recipients of $2 \times 10^{7} \mathrm{CAR}$ T-cells $\mathrm{m}^{-2}$ than those receiving $1 \times 10^{8}$ cells $\mathrm{m}^{-2}$ or greater ${ }^{192}$.

Conversely, within a dose range of $5 \times 10^{5}$ to $1 \times 10^{7} \mathrm{CAR}$ T-cells kg-1 Gardner et al. did not observe a relationship between dose and CAR T-cell expansion in children with r/r B-ALL ${ }^{253}$, while among patients receiving tisagenleuceucel for $\mathrm{r} / \mathrm{r}$ B-NHL, no relationship between dose and exposure $\left(\mathrm{AUC}_{0-28 \mathrm{~d}}\right.$ and $\left.\mathrm{C}_{\max }\right)$ was seen within a total dose range of $6 \times 10^{7}$ to $6 \times$ $10^{8}$ cells ${ }^{254}$. 
In B-cell malignancies including B-ALL, B-NHL, CLL and myeloma, CAR T-cell exposure has been associated with clinical response ${ }^{11} 154$. For example, among both children receiving tisagenlecleucel for B-ALL and adults receiving axicabtagene ciloleucel for B-NHL, $\mathrm{C}_{\max }$ and $\mathrm{AUC}_{0-28 \mathrm{~d}}$ were significantly greater in responders than in non-responders ${ }^{11}{ }^{154}$. An association between $\mathrm{C}_{\max }$ and clinical response rate was also reported among recipients of anti-BCMA CAR T-cells for myeloma ${ }^{224}{ }^{255}$. In contrast, the JULIET trial of tisagenlecleucel in adults with B-NHL did not report an association between $\mathrm{AUC}_{0-28 \mathrm{~d}}$ and clinical response ${ }^{76}$.

Both $\mathrm{C}_{\max }$ and $\mathrm{AUC}_{0-28 \mathrm{~d}}$ reflect short-term $\mathrm{CAR} \mathrm{T}$-cell exposure, but for some malignancies, long-term CAR T-cell persistence may be predictive of durable remission ${ }^{133}$. CAR T-cell persistence is thought to be particularly important in B-ALL, as persistence correlates with improved disease-free survival ${ }^{105123154}$, and CD19+ leukaemic relapse is a frequent occurrence among individuals who lose anti-CD19 CAR T-cell persistence ${ }^{256}$. In B-NHL, the relationship between CAR T-cell persistence and long-term outcomes is less clear: the majority of axicabtagene ciloleucel recipients for B-NHL remaining free of disease at 24 months show recovery of normal B-cell populations ${ }^{78} 245$, suggesting that CAR T-cell persistence is not a pre-requisite for durable remission in this disease.

\subsection{Dose-toxicity relationship}

The onset of CRS is typically within one to two weeks after CAR T-cell administration, coinciding with brisk CAR T-cell expansion ${ }^{113}{ }^{127}$. Several studies report that CAR T-cell recipients who developed severe $\mathrm{CRS}$ had higher $\mathrm{C}_{\max }$ and/or $\mathrm{AUC}_{0-28 \mathrm{~d}}$, compared to those who did not 586224254 , although not all trials have observed this ${ }^{11} 128$.

Neurotoxicity (ICANS), can occur concurrently with CRS or in isolation. Anti-CD19 CAR T-cell trials in both $\mathrm{r} / \mathrm{r}$ B-ALL and B-NHL have reported that severe ICANS is associated with higher $\mathrm{AUC}_{0-28 \mathrm{~d}}$ and/or $\mathrm{C}_{\max } 117186113117128131$. In contrast, among recipients of tisagenlecleucel for r/r B-ALL, no significant association between CAR T-cell expansion and severe neurological adverse events was reported ${ }^{154}$. The discrepancy between trials may be accounted for by differences in toxicity grading and management protocols, different product or disease characteristics, or because blood CAR T-cell levels may not always reflect expansion of, and cytokine production by, CAR T-cells within tissues. 


\subsubsection{Can a therapeutic window be identified}

In B-cell malignancies, in vivo CAR T-cell expansion is a major determinant of exposure, and a lack of expansion is associated with lack of clinical response. There appears to be a 'threshold' dose of CAR T-cells, beneath which robust expansion, and clinical response, is unlikely. This threshold is likely to vary between products and treatment indications, and with patient factors, such as tumour burden and immune function. Above this threshold, escalating the CAR T-cell dose may have little additional benefit in terms of response rate, but could potentially add to the risk of severe CRS or ICANS.

Identifying a CAR T-cell dose that is sufficient to allow robust CAR T-cell expansion and efficacy in the majority of recipients, without undue elevation of severe toxicity risk, may be challenging. Figure 3.3 illustrates one model, in which the therapeutic window can be conceptualised as the range between a minimum effective dose (MED) and a maximum tolerated dose (MTD). This window may be difficult to determine, may be narrow, and is likely to depend upon disease indication, product and patient characteristics ${ }^{133}$.

The relationship between initial CAR T-cell dose and long-term persistence of the infused CAR T-cell, and between persistence and relapse-free survival is poorly defined, and is also likely to vary by CAR T-cell construct and by clinical indication.

\subsubsection{Risk-stratified dosing}

One way to overcome a narrow therapeutic window for CAR T-cell therapies is to stratify dose according to toxicity risk ${ }^{113}$. Reasoning that a heavy disease burden was associated with greater CAR T-cell expansion and toxicity risk, Turtle et al. stratified CAR T-cell doses in adults with B-ALL according to pre-treatment bone marrow leukaemia burden, reporting retention of anti-tumour efficacy without excessive toxicity ${ }^{5}$. Similarly, doses of CAR Tcells directed against the plasma cell antigen BCMA have been stratified based on bone marrow myeloma burden 224252 . While lymphoma disease bulk has been associated with elevated CRS and ICANS risk ${ }^{257}$, it is not yet clear whether or not modifying CAR T-cell dose can retain efficacy while reducing toxicity risk in $\mathrm{B}-\mathrm{NHL}^{86} 113$. 
Various pre-treatment biomarkers have been associated with elevated CRS and ICANS risk, including serum lactate dehydrogenase (LDH), CRP, ferritin, and markers of endothelial activation 71113130133219 . Whether these can help to stratify CAR T-cell dosing is not yet known.

Figure 3.3 Selecting an optimal CAR T-cell dose

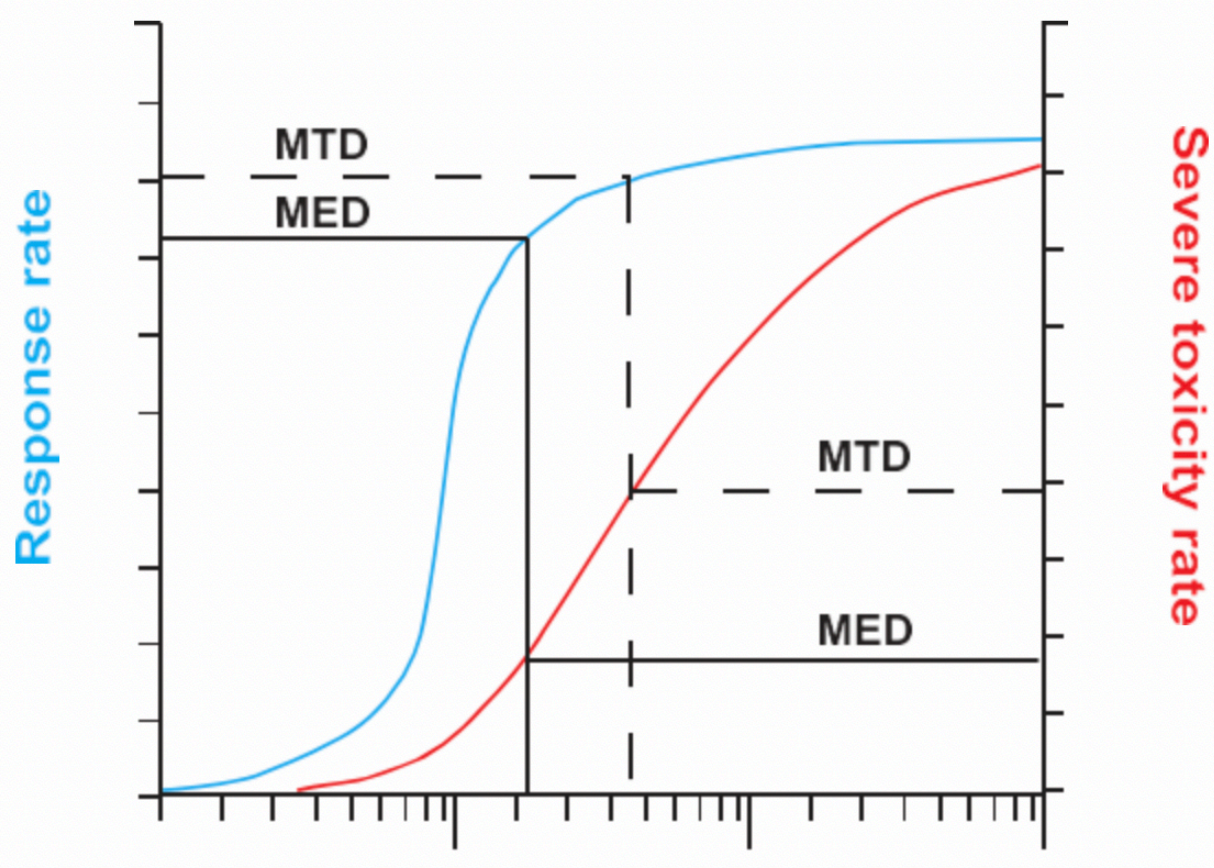

CAR T-cell dose

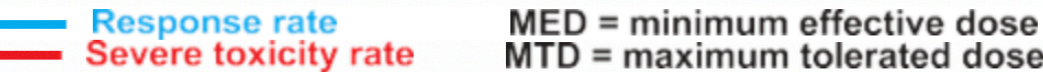

Selecting an optimal chimeric antigen receptor (CAR) T-cell dose. Beneath a certain dose threshold, CAR T-cells may fail to expand within the recipient, and both response and severe toxicity are unlikely. Above this threshold, clinical efficacy may increase rapidly until a minimum effective dose is reached, beyond which there may be little further improvement in response rate with increasing dose. In contrast, the rate of severe toxicities may continue to rise as the dose is increased. Phase I trial designs that seek to determine the minimum effective dose as well as the maximum tolerated dose may facilitate selection of a dose that maximises response rate without undue toxicity risk.

\subsubsection{Repeated dosing}

In the face of marked inter-individual variability in CAR T-cell pharmacokinetics and toxicity rates, the notion of within-patient dose escalation is appealing, allowing each 
recipient to first receive a low CAR T-cell dose, to be observed for toxicities, and to receive higher doses if no toxicity is observed. Limitations of this approach include the potential need to repeatedly administer lymphodepleting chemotherapy, with its inherent toxicities.

Omitting lymphodepletion may be particularly problematic for repeated CAR T-cell dosing, as repeated CAR T-cell exposure might provoke immunological responses against the CAR, limiting subsequent expansion and persistence 5104105 . This may be mitigated by employing humanised rather than murine scFvs ${ }^{258} 259$ within CAR T-cell constructs, but even fully humanised constructs can elicit immune responses ${ }^{94}$.

\subsubsection{Dose escalation trials}

\subsubsection{Dosing in anti-CD19 CAR T-cell trials registered on clinicaltrials.gov}

A systematic search on clinicaltrials.gov for anti-CD19 CAR T-cell trials was carried out, in order to identify the range of CAR T-cell doses used in anti-CD19 clinical trials.

\section{Method:}

- Supplementary data provided from a CAR T-cell review article by Hartman et al. listing all anti-CD19 CAR T-cells registered on clinicaltrials.gov until the end of 2016 was accessed ${ }^{194}$.

- The anti-CD19 CAR T-cell dose for each trial was recorded if dosing information accessible on clinicaltrials.gov. The dosing information included the starting dose for the trial, the maximum tolerated dose reached (if applicable) and the selected dose for a Phase II study (if applicable).

- If dosing information not accessible, an email was sent to the corresponding investigator on clinicaltrials.gov, requesting dosing information for that trial.

- On 06/12/2018 a search on clinicaltrials.gov for anti-CD19 CAR T-cell trials was carried out using the following search terms: $\boldsymbol{C A R} \boldsymbol{T}$-cells, $\boldsymbol{C D 1 9}$ and $\boldsymbol{B}$-cell to obtain a list of clinical trials.

- All trials that had been previously included in the supplementary data obtained from Hartmann et $a l^{194}$, were removed from this list of trials. 
- For the remaining anti-CD19 CAR T-cell trials obtained from this search, the CAR Tcell dose for each trial was recorded if the dosing information was available on clinicaltrials.gov.

- If dosing information was not accessible, an email requesting this information was sent to the corresponding investigator on clinicaltrials.gov.

The process for obtaining anti-CD19 CAR T-cell trial doses is summarised in 
Figure 3.4 CONSORT diagram for anti-CD19 CAR T-cell trial dosing information obtained from clinical trials.gov




The results from the anti-CD19 CAR T-cell trial dosing obtained from these searches of clinicaltrials.gov are recorded in Figure 3.5.

Figure 3.5 Doses from anti-CD19 CAR T-cell trials registered on clinicaltrials.gov

\section{Anti-CD19 CAR T-cell dose}

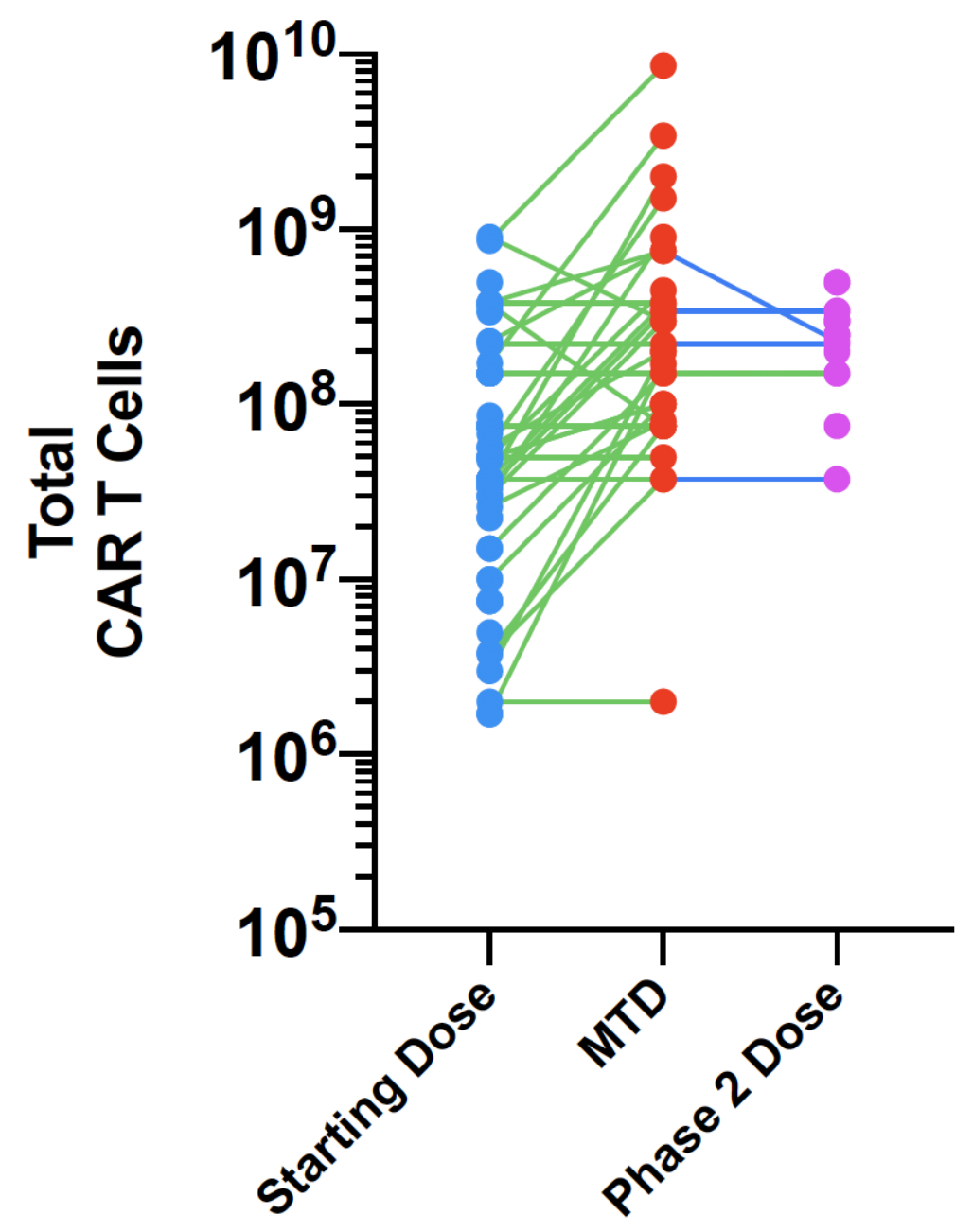

Dose Escalation Range

In the 89 trials identified from the search of clinicaltrials.gov with anti-CD19 CAR T-cell dosing information available; the starting dose (blue dots), Maximum tolerated dose (MTD) if available (red dots) and Phase 2 dose if available (purple dots), are plotted on this graph. 
Each green line connects the starting dose from a specific trial (a blue dot) to the MTD for that trial (a red dot), or to a Phase 2 dose for that trial (a purple dot). Each blue line connects an MTD from a specific trial (a red dot) to a Phase 2 dose for that trial (a purple dot). The y axis uses a logarithmic scale for total CAR T-cell dose.

\subsubsection{2 ' $3+3^{\prime}$ dose escalation trial design}

Many CAR T-cell dose escalation trials use a ' $3+3$ design,' whereby up to three subjects are treated at a given dose, and the dose escalated only if fewer than a pre-defined number of dose limiting toxicities (DLTs) occur 105252260261 . A review of CAR T-cell trials registered at ClinicalTrials.gov up to the end of 2018, found that the typical dose escalation trial covered a two-log (100-fold) dose range, typically within the range $10^{6}-10^{10}$ total CAR T-cells per subject (see Figure 3.5) ${ }^{194}$. Doses are frequently escalated in half-log (approximately 3-fold) to $\log$ (10-fold) steps 105192224234261 . Dose expansion cohorts may be employed to further characterise toxicity profiles and to aid design of subsequent efficacy trials 192224255 .

A number of factors impact dose escalation approaches, including lymphodepleting regimen, disease indication and manufacturing process ${ }^{1186128194}$. For example, Turtle et al. conducted a separate anti-CD19 CAR T-cell dose escalation following incorporation of fludarabine into the lymphodepletion regimen, observing greater CAR T-cell expansion and higher clinical response rates in the fludarabine-treated cohorts ${ }^{86}$. Many dose escalation trials now incorporate uniform fludarabine and cyclophosphamide lymphodepletion at each dose step ${ }^{66}$ 82224234

Manufacturing changes also have potential to impact the safety and efficacy of a CAR T-cell product ${ }^{94251}$, and may necessitate additional dose finding studies if product comparability cannot be assured ${ }^{262}$.

\subsubsection{Beyond MTD}

Dose escalation trials classically aim to identify a MTD by assessing for dose limiting toxicities (DLTs) 192224234 . However, if there is a 'threshold' dose above which CAR T-cells are likely expand within the recipient, the MTD may be higher than the dose needed for maximal clinical efficacy ${ }^{263}$. Selecting an unnecessarily high CAR T-cell dose could 
adversely affect both manufacturing feasibility and toxicity risk. Instead, the determination of a Minimum Effective Dose (MED), taking account of pharmacokinetic and/or efficacy data as well as toxicity rates, may be a preferable goal for dose-finding CAR T-cell trials ${ }^{264}$.

Phase I trial designs that incorporate both safety and efficacy data have been proposed for adoptive cellular therapies. These include Toxicity Equivalence Range (TEQR) and Modified Toxicity Probability Interval (mTPI) designs ${ }^{265,266}$. Alternatively, Chiuzan et al. propose a two stage design, in which an initial dose escalation stage incorporating a surrogate outcome (such as pharmacokinetics) is followed by second adaptive randomisation stage, which assigns subjects to all dose levels showing signals of efficacy, within safety constraints defined during stage one ${ }^{267}$. A general limitation is that inter-individual variability in CAR T-cell products and their pharmacokinetics, and a potentially narrow therapeutic window, may limit the ability to determine a MED within small phase I CAR T-cell trials ${ }^{268}$.

\subsubsection{Cost effectiveness and off-the-shelf-products}

List prices for the licensed autologous CAR T-cell products axicabtagene ciloleucel and tisagenlecleucel are high, at USD $\$ 373,000$ and $\$ 475,000$, respectively ${ }^{269}$. In addition to this, funders must consider the costs of T-cell harvest procedures, lymphodepleting chemotherapy, CAR T-cell administration, monitoring, toxicity management, and, for some recipients, immunoglobulin replacement for B-cell aplasia ${ }^{270}$. These costs are likely to limit the availability of CAR T-cell therapies in the short term, at least pending longer-term follow-up and the outcomes of phase III trials.

Optimising the dose has the potential to maximise the feasibility of product manufacture, and possibly to reduce toxicity risk. However, the need to produce and test a personalised cellular product limits economy of scale. One solution is to develop 'off the shelf' CAR-transduced cellular products, in which CAR-expressing cells are manufactured from a pool of healthy donors, from umbilical cord blood, or from a cell line. The risk of donor-versus-recipient 'graft versus host disease' due to allogeneic T-cell administration can be addressed through a variety of strategies, such as by selecting tissue type-matched donors, by expressing CARs in cells that lack a conventional T-cell receptor (such as natural killer cells or innate-like Tcells), or by deleting the T-cell receptor locus ${ }^{271-274}$. If successful, 'off-the-shelf' CARtransduced cellular therapies may overcome a many of the logistical, feasibility, cost and 
product variability issues of autologous CAR T-cell therapies, although each 'off-the-shelf' product will require dose optimisation.

\subsection{Conclusion}

As cell-based gene-transduced therapies, CAR T-cells exhibit unusual pharmacokinetic properties, including variable degrees of expansion within the recipient, and variable longterm persistence. Selecting an optimal dose requires consideration of underlying disease, tumour burden and lymphodepleting chemotherapy, as well as characteristics of the manufactured CAR T-cell product itself.

Clinical experience of autologous CAR T-cell therapies other than those directed against CD19 is limited, and there are many unresolved questions regarding dosing. For example, inter-individual variation in pharmacokinetics is considerable, and the factors associated with this are still under investigation. CAR-transduced cells directed against alternative target antigens, manufactured using abbreviated culture methods, transduced to express additional proteins, manufactured from cell lines or from allogeneic donors, or administered directly into tumour sites rather than intravenously may exhibit very different pharmacokinetic characteristics to those outlined in this review. Tools such as alternative phase I trial designs, CAR T-cells with intrinsically reduced potential for toxicity, and strategies to stratify CAR T-cell dosing based on product characteristics or disease burden, could all help clinicians and researchers optimise dosing and, in the long-term, widen the therapeutic window and the availability of this emerging cancer immunotherapy. 


\title{
4. Chimeric antigen receptor T-cells in New Zealand: Challenges and Opportunities
}

George P, Giunti G, ...... Weinkove R.

This chapter is a manuscript in advanced draft format for submission to the New Zealand Medical Journal as a 'viewpoint article.'

\begin{abstract}
Chimeric antigen receptor (CAR) T-cells are an emerging modality of cancer therapy. CAR T-cells are lymphocytes that have been engineered to express a synthetic receptor, which enables them to target tumour cells ${ }^{275}$. Internationally, CAR T-cell therapies are becoming a standard of care for the treatment of certain relapsed and refractory B-cell lymphomas and leukaemias; with promising results for other malignancies, including myeloma ${ }^{12,13276 .}$
\end{abstract}

As both a personalised cell and a gene therapy, CAR T-cells present unique regulatory requirements, while the logistics of CAR T-cell production and delivery, and the potential for adverse events, demand specific preparation by treatment centres. The nature of CAR T-cell therapies may raise ethical and cultural questions, which need to be addressed to ensure equity of access and outcomes.

We recently established local Good Manufacturing Practice (GMP) CAR T-cell manufacture, and commenced enrolment to New Zealand's first CAR T-cell trial, ENABLE (ClinicalTrials.gov reference NCT04049513) ${ }^{277}$. This involved close liaison with regulators and stakeholders, and multidisciplinary input to facilitate the safe clinical delivery of CAR Tcell therapies. This Viewpoint article outlines the current regulatory landscape for CAR Tcell therapies in New Zealand, summarises the institutional preparations required, and discusses potential avenues to broaden access to this new modality of cancer therapy to New Zealand.

\subsection{CAR T-cell manufacturing and logistics}

\subsubsection{Leukocyte harvest and leukapheresis}

Figure 4.1 illustrates a typical CAR T-cell manufacturing process. In brief, leukocytes are harvested from blood, typically via a leukapheresis procedure. Following purification of Tcells, a new gene (transgene) encoding the CAR is introduced, typically using a lentiviral or 
retroviral vector ${ }^{278}$. The CAR T-cells are expanded, then cryopreserved, while quality control testing is undertaken ${ }^{203}$.

The two commercially-licensed autologous CAR T-cell products tisagenlecleucel and axicabtagene ciloleucel, require a leukapheresis procedure ${ }^{278}$ 279. The New Zealand Blood Service (NZBS) is a national service, routinely performing leukapheresis for haematopoietic stem cell harvest across NZ. NZBS is accredited by Medsafe for collection of 'therapeutic cells by apheresis', and has conducted leukapheresis procedures for cellular therapy trials, including ENABLE 277,280,277 281282 . Leukapheresis product testing for key blood-borne viruses can be conducted at the Medsafe-licensed NZBS Donation Accreditation Laboratory. While manufacturers of commercial and investigational CAR T-cell products will impose specific requirements for patient identification, infectious agent testing, leukapheresis conduct, and product labelling and shipping, in our opinion, the NZBS leukapheresis service is well placed to conduct leukapheresis procedures for CAR T-cell manufacture within New Zealand in the future.

\subsubsection{Laboratory manufacture and release of CAR T-cells}

Although not viable except under specialised culture conditions, or within the recipient, CAR T-cells are defined as genetically-modified organisms (GMOs) by New Zealand legislation. CAR T-cell manufacture is regulated by the Environmental Protection Agency (EPA) under the Hazardous Substances and New Organisms (HSNO) Act 1996. Before granting approval to manufacture CAR T-cells, the EPA sought assurance that the cells could not escape into the environment. Meeting containment requirements required modifications to our GMP manufacturing facility and to its operating procedures. The Ministry of Primary Industries (MPI) inspects and audits the facility.

EPA approval to release the CAR T-cell product to the clinical facility (for administration to patients) was required. Measures to assure safe release include rigorous product release criteria, product labelling according to ISBT standards, and stringent procedures for safe packaging, transport, administration and disposal. Notably, although classified as a GMO in New Zealand, CAR T-cells are not viable outside the intended or specialised culture conditions; current legislation does not distinguish between CAR T-cells and viable organisms, such as GMO plants, seeds or animals. 
Figure 4.1 Visual Summary of CAR T-cell manufacturing process

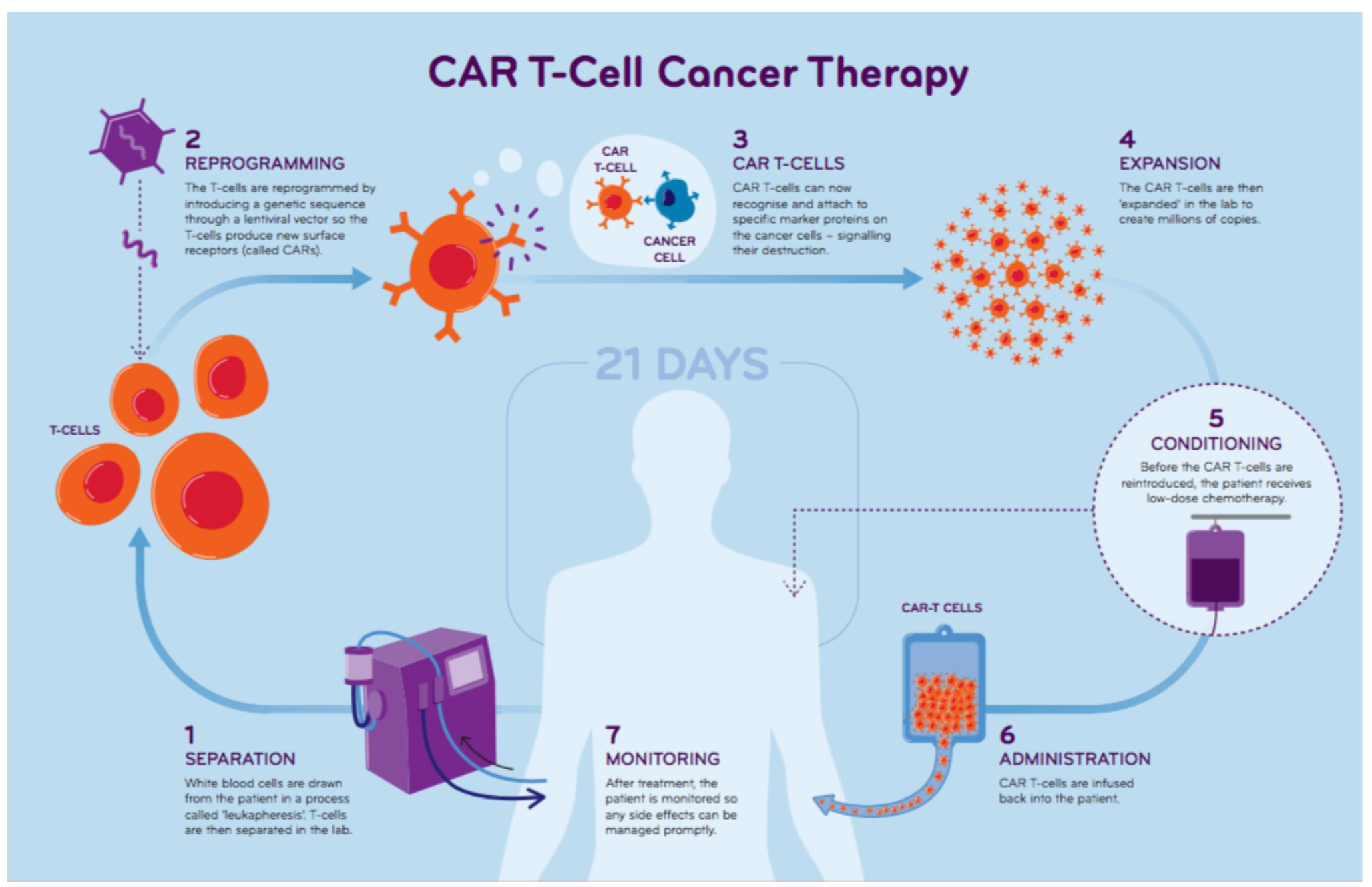




\subsubsection{Shipping and product traceability}

Once a CAR T-cell product has been manufactured and met specific 'release criteria', the cells are retrieved from the liquid nitrogen storage tank and prepared for delivery to the clinical centre for administration. At all stages, rigorous identity checks using three independent subject and product identifiers are performed and captured on designated forms, to ensure the "Chain of Custody" is maintained, and that the right patient receives the right product. Unused CAR T-cell vial(s) are returned to the manufacturing facility for safe disposal. The agencies involved in regulating CAR T-cell manufacture, release and delivery in New Zealand are summarised in Table 4.1.

\subsubsection{Cultural considerations}

Significant health inequalities between Māori and non-Māori persist, with higher cancerrelated mortality in Māori ${ }^{283}$ and cancer becoming a larger important contributor to mortality inequalities over time ${ }^{284}$. Researchers in New Zealand have an obligation to consider the degree to which they can contribute to improving Māori health outcomes and Māori review of research is an ethical and legislative requirement for research carried out within District Health Boards in New Zealand ${ }^{285} 286$. Furthermore Māori regard tissue and genetic material as Taonga (of spiritual value) and the application of genetic engineering may be of concern. However, where there could be direct health benefits for Māori and their whānau, many will be understanding of this application ${ }^{287} 288$. Because CAR T-cell therapies typically utilise autologous cells, ethnicity should not present a barrier to treatment. This contrasts with unrelated allogeneic stem cell transplantation, which requires a tissue type-matched donor ${ }^{289}$. However, complex new technologies have the potential to increase health disparities between

groups ${ }^{290}$. Overall, a Māori consultation process is therefore essential for CAR T-cell related research and work.

Māori advisory groups consult on research projectS; for our own trial (ENABLE), consultation was sought at an early stage during study development. Following consultation, a Participant Information and Consent Form incorporating a short lay summary, in both English and te reo, and a visual representation of the CAR T-cell treatment process, was developed. To reduce the risk that distance from the treatment centre impeded study access, an agreement was reached with a national cancer charity to support travel costs for study participants, if not deemed eligible for the National Travel Assistance scheme. Finally, 
liaison with a Māori clinician and researcher familiar with managing patients with haematological malignancies was carried out, and will be an ongoing strategy to help facilitate Māori participation in CAR T-cell research in the future.

\subsection{Clinical Delivery of CAR T-cell therapy}

\subsubsection{CAR T-cell toxicity management working group}

Anti-CD19 CAR T-cell therapy can cause severe treatment related toxicities, some of which can be specific to this modality of therapy ${ }^{109}$. In particular, Cytokine Release Syndrome (CRS) and Immune Effector Cell Associated Neurotoxicity Syndrome (ICANS) are well recognised toxicities following anti-CD19 CAR T-cell therapy ${ }^{109}$. There are now consensus guidelines to grade CRS and ICANS published by the American Society for Transplantation and Cellular Therapy (ASTCT) ${ }^{126}$. Reported rates of severe ( $\geq$ grade 3 ) CRS and ICANS of the two approved anti-CD19 CAR T-cell therapies, axi-cel and tisagenlecleucel in the pivotal Phase 2 ZUMA-1 and JULIET trials, were $13-22 \%$ and $12-28 \%$ respectively ${ }^{11} 76$. There are published, expert consensus recommendations for the recognition and management of both CRS and ICANS, which are constantly evolving based on emerging evidence in this rapidly developing field ${ }^{71} 109$.

In recognition of the need for specific risk mitigation measures to be taken in order to safely deliver CAR T-cell therapy, the CAR T-cell trial team in Wellington convened a CAR T-cell toxicity working group, named the CARTOX group. This group is composed of Haematologists, a Neurologist, an Intensive Care Physician, an Immunologist and Haematology nursing representatives. The CARTOX group has led localisation of CRS and ICANS clinical recognition and management pathways utilised at the CAR T-cell treatment site. These local guidelines were based on current international consensus recommendations ${ }^{109}$ and advice from experts in the field (Neelapu and Turtle, personal communication). The group provides a multidisciplinary overview of CAR T-cell toxicity education and training material for relevant clinical staff. In the future, the CARTOX group will be consulted in any cases where severe treatment related toxicities are encountered. As more patients are treated with CAR T-cell therapy, the CARTOX group will review the clinical pathways and may recommend amendments based on clinical experience or emerging evidence and guidance in this field. Based on feedback from international CAR T-cell 
treating clinicians and from our own initial experience, we recommend the implementation of a CAR T-cell toxicity group at any centre treating patients with CAR T-cell therapy.

\subsubsection{CAR T-cell toxicity education}

In order to safely deliver CAR T-cell therapy, it is essential that clinical staff receive appropriate training in the recognition and management of CAR T-cell related toxicities. Risk Evaluation and Mitigation Strategies (REMS), which are mandated by the manufacturers of commercially licensed CAR T-cell products as a condition of FDA approval, require CAR Tcell treating centres to be certified to ensure the appropriate training and knowledge assessment for clinical staff is in place to allow the safe delivery of CAR T-cell therapies ${ }^{139}$ 140. This encompasses areas such as prescribing, dispensing and administering CAR T-cells; along with monitoring for, recognising and treating CAR T-cell related toxicities ${ }^{139}{ }^{140}$. In Wellington, the ENABLE Trial team designed a competency based multiple choice assessment on CAR T-cell related toxicities for all haematology clinical staff and appropriate nursing staff in the Patient at Risk and ICU teams. Furthermore, a specific CAR T-cell toxicity one page 'Quicksheet' was produced and made available to all clinical staff treating a CAR T-cell patient. We recommend that clinical staff receive regular educational updates, on at least a quarterly basis, to familiarise them with CAR T-cell related toxicities.

\subsubsection{PHARMAC provision of tocilizumab}

Tocilizumab, a monoclonal antibody against the IL-6 receptor, has been shown to be effective in the management of anti CD19 CAR T-cell mediated toxicities including CRS and ICANS, and does not appear to adversely affect anti-tumour responses ${ }^{71,136}$. Although tocilizumab is not currently licensed by Medsafe in New Zealand for the treatment of CRS or ICANS post CAR T-cell therapy, it is registered by the US Food and Drugs Administration (FDA) for the treatment of CAR T-cell induced $\mathrm{CRS}^{138}$ and is recommended in consensus international treatment algorithms for CRS and ICANS ${ }^{109,71} 114$. The REMS strategy for axicel requires that at least two doses of tocilizumab are available on site for each patient when they are treated ${ }^{140}$. Given the necessity for access to tocilizumab following CAR T-cell therapy, a clinician led application to PHARMAC to fund up to 3 doses of tocilizumab to treat CRS and/or ICANS following CAR T-cell treatment on the ENABLE Trial was made, with subsequent approval received on February $1^{\text {st }} 2019^{291}$. 
Table 4.1 Regulatory requirements for manufacturing and delivering CAR T-cell therapy within New Zealand

\begin{tabular}{|c|c|c|c|}
\hline Regulatory Body & Application to CAR T-cell therapy & $\begin{array}{l}\text { New Zealand } \\
\text { Legislation or } \\
\text { guidance }\end{array}$ & Standards to be met \\
\hline $\begin{array}{l}\text { Health and Disability } \\
\text { Ethics Committee } \\
\text { (HDEC) }\end{array}$ & $\begin{array}{l}\text { Approval required before conducting all CAR T-cell } \\
\text { trials }\end{array}$ & $\begin{array}{l}\text { Medicines Act 1981, the } \\
\text { Guideline on the Regulation of } \\
\text { Therapeutic Products in New } \\
\text { Zealand * }\end{array}$ & $\begin{array}{l}\text { Conduct according to CHMP } \dagger \text { guidance document EMA/CHMP/ICH/135/95 } \\
\text { Guideline for Good Clinical Practice E6(R2) to meet International Conference on } \\
\text { Harmonisation Good Clinical Practice (IHC GCP) criteria }\end{array}$ \\
\hline $\begin{array}{l}\text { Gene Technology } \\
\text { Advisory Committee } \\
\text { (GTAC) } \ddagger\end{array}$ & $\begin{array}{l}\text { As a cell therapy comprising genetically manipulated } \\
\text { cells, all CAR T-cell trials require GTAC approval }\end{array}$ & $\begin{array}{l}\text { Section } 30 \text { of the Medicines } \\
\text { Act } 1981\end{array}$ & $\begin{array}{c}\text { In relation to the investigational CAR T-cell product, to ensure: } \\
\text { Scientific rationale for potential clinical benefit } \\
\text { Safety and toxicity data acceptable } \\
\text { Qualifications and experience of investigators } \\
\text { investigators }\end{array}$ \\
\hline $\begin{array}{l}\text { Research Advisory } \\
\text { Group Māori }\end{array}$ & $\begin{array}{l}\text { Māori consultation is an ethical and legislative } \\
\text { requirement for research carried out within District } \\
\text { Health Boards in New Zealand. }\end{array}$ & $\begin{array}{l}\text { Te Ara Tika } \\
\text { Guidelines for Māori research } \\
\text { ethics, Health Research } \\
\text { Council New Zealand }\end{array}$ & $\begin{array}{l}\text { Research proposals to be developed in consultation with Māori. Research should be } \\
\text { reviewed and approved by Māori before commencing }\end{array}$ \\
\hline $\begin{array}{l}\text { Environmental } \\
\text { Protection Agency } \\
\text { (EPA) }\end{array}$ & $\begin{array}{l}\text { Approval required to: } \\
\text { classified as GMOs (Genetically Modified } \\
\text { Organisms) in containment } \\
\text { containment to treatment delivery site and to } \\
\text { clinical laboratory for safety testing }\end{array}$ & $\begin{array}{l}\text { Hazardous Substances and } \\
\text { New Organisms (HZNO) Act } \\
1996\end{array}$ & $\begin{array}{l}\text { - Demonstrate satisfactory containment level in place to } \\
\text { prevent escape of the GMOs into the environment } \\
\text { sustaining population outside of containment when released } \\
\text { Ensure necessary controls to mitigate potential risk are in } \\
\text { place to release GMO from containment }\end{array}$ \\
\hline Medsafe & $\begin{array}{l}\text { License to manufacture cell therapy product (pack, label } \\
\text { and sell by wholesale) } \\
\text { Accreditation of clinical leukapheresis service } \S\end{array}$ & $\begin{array}{l}\text { PIC/S } \rrbracket^{\uparrow} \text { Guide for Good } \\
\text { Manufacturing Practice for } \\
\text { Medicinal Products }\end{array}$ & $\begin{array}{l}\text { Ensuring the manufacturing facility and manufacturing procedures (including batch } \\
\text { manufacturing records and product release criteria) meet Good Manufacturing } \\
\text { Practice (GMP) standards } \\
\text { Leukapheresis service audited against the code of GMP }\end{array}$ \\
\hline
\end{tabular}

*Under this legislation all clinical trials in NZ must receive HDEC approval

†HMP (The Committee for Medicinal Products for Human Use) is the European Medicines Agency's (EMA) committee responsible for human medicines

$\ddagger$ A committee maintained by the Health Research Council (HRC) of New Zealand to consider applications for trials involving gene or other biotechnology therapies

$\S$ Clinical leukapheresis performed by the New Zealand Blood Service which is Medsafe accredited

I PIC/S ( Pharmaceutical Inspection Convention Pharmaceutical Inspection Co-operation Scheme 


\subsubsection{Patient Education}

It is essential that patients receive clear and coherent education on the process and on the potential adverse effects of CAR T-cell therapy ${ }^{292}$. It is widely recommended that patients should remain within 30 minutes of the CAR T-cell treating centre alongside a support person for at least 28 days following CAR T-cell therapy ${ }^{137}$. For the two commercially approved anti-CD19 CAR T-cell products, some patients who receive tisagenlecleucel may receive treatment as an outpatient, whereas for patients receiving receiving axi-cel, it is mandated they should remain an inpatient for at least 7 days following infusion ${ }^{63} 64$. Therefore, many patients will be out of hospital for at least some time during the first 21 days following infusion, the period of highest risk for anti-CD19 CAR T-cell related toxicities ${ }^{109}$. Hence, it is essential that CAR T-cell recipients receive education on CAR T-cell related toxicities and should receive a manufacturer provided wallet card describing all symptoms that warrant immediate evaluation, such as a fever or speech difficulties ${ }^{165}$. After this 28 day period, most CAR T-cell recipients will return to their home regions, which may be a long way from their CAR T-cell treatment centre. In many cases, the clinicians looking after the patient in their home region will not have CAR T-cell treating experience or expertise, so ensuring patients are well educated and have access to clear and accurate information to pass on to other healthcare professionals, is essential ${ }^{292}$. For the ENABLE Trial, all CAR T-cell recipients receive a wallet emergency card and a discharge summary sheet, outlining possible adverse effects to be aware of and the criteria to seek medical advice with key contact details provided. It is our recommendation that these two patient education materials are essential and should be provided to all patients receiving CAR T-cell therapy.

\subsubsection{International Cellular Therapies Registries}

As CAR T-cell therapies are a relatively new treatment modality, there is a possibility that low incidence, or late onset, toxicities will emerge, such as second malignancies or adverse pregnancy outcomes. Cellular therapy registries provide an important framework for longterm follow-up and detection of such risks ${ }^{293}$.

The Center for International Blood and Marrow Transplant Research (CIBMTR) opened a Cellular Therapies Registry in June 2016, which aims to standardise CAR T-cell toxicity 
reporting and data collection ${ }^{294}$. Participation in the CIBMTR Cellular Therapy Registry is open to CAR T-cell treatment centres worldwide, provided ethical approval for data collection and sharing is in place. Within Australasia, the Australian Bone Marrow Transplant Recipient Registry (ABMTRR) has recorded outcomes of hematopoietic stem cell transplantation since 1992, ${ }^{295}$ and has expanded its remit to collect data from CAR T-cell recipients ${ }^{13}$.

Table 4.2 Opportunities and challenges for CAR T-cell therapy in New Zealand

\begin{tabular}{|l|l|}
\hline Challenges & Opportunities \\
\hline Limited CAR T-cell track record & $\begin{array}{l}\text { NZ Blood Service and stem cell transplant } \\
\text { centres are national networks, and well-placed } \\
\text { for CAR T-cell delivery }\end{array}$ \\
\hline $\begin{array}{l}\text { Geographical distance from most large-scale } \\
\text { manufacturing centres }\end{array}$ & $\begin{array}{l}\text { Local manufacturing capability already exists, } \\
\text { and can be expanded }\end{array}$ \\
\hline $\begin{array}{l}\text { Current cost of commercial CAR T-cell } \\
\text { therapies is high }\end{array}$ & $\begin{array}{l}\text { CAR T-cell therapies are a 'one-off' therapy; } \\
\text { costs will decline with competition }\end{array}$ \\
\hline $\begin{array}{l}\text { Limited availability of drugs to treat toxicities } \\
\text { Numerous regulatory agencies involved }\end{array}$ & $\begin{array}{l}\text { PHARMAC provides a mechanism for funding } \\
\text { Medsafe regulations are harmonised with EMA; } \\
\text { clinical trials provide regulatory experience }\end{array}$ \\
\hline $\begin{array}{l}\text { Cultural concerns about application of gene- } \\
\text { modified cells to treat cancer }\end{array}$ & $\begin{array}{l}\text { Consultation and a commitment to equity can } \\
\text { ensure all New Zealanders benefit }\end{array}$ \\
\hline Lack of clinical experience & $\begin{array}{l}\text { ENABLE trial demonstrates feasibility of CAR } \\
\text { T-cell delivery \& toxicity management }\end{array}$ \\
\hline
\end{tabular}

\subsection{Conclusion}

CAR T-cell therapy is shifting the treatment paradigm of $\mathrm{r} / \mathrm{r} \mathrm{B}$-cell malignancies internationally. However, there are a number of regulatory, clinical, logistical and financial challenges to implementing this type of therapy. In this view point article, we have discussed the process of delivering CAR T-cell therapy within New Zealand, through setting up the ENABLE trial. In New Zealand, CAR T-cell manufacturing capability, regulated by Medsafe and harmonised with the European Medicines Agency, now exists, and can be expanded to meet demand for CAR T-cell treatment within New Zealand. The ability to manufacture CAR T-cells locally within New Zealand, may have logistical advantages for the delivery of CAR T-cells in the COVID-19 era ${ }^{296}$. The process of delivering CAR T-cell therapy within 
NZ, has the advantage of working with international networks such as the NZBS, to help achieve its aims. Ongoing consultation with Māori advisory groups will help to ensure that CAR T-cell therapy is an equitable treatment of potential benefit to all New Zealanders. Localisation of clinical and toxicity management protocols for the ENABLE trial, has demonstrated the feasibility of the clinical delivery of CAR T-cell therapy, and this will continue to develop as this trial treats more participants. Overall, we believe that New Zealand is in a good position to develop and grow both investigational and commercial CAR T-cell therapies in the future. 


\title{
5. Third-generation anti-CD19 chimeric antigen receptor T-cells incorporating a TLR2 domain for relapsed or refractory B-cell Iymphoma: a phase I clinical trial protocol (ENABLE)
}

\section{BMJ Open 2020 Feb 9;10(2):e034629.}

Philip George, Nathaniel Dasyam, Giulia Giunti, Brigitta Mester, Evelyn Bauer, Bethany Andrews, Travis Perera, Tess Ostapowicz, Chris Frampton, Peng Li, David Ritchie, Catherine M. Bollard, lan F. Hermans, Robert Weinkove

\begin{abstract}
Introduction: Autologous T-cells transduced to express a chimeric antigen receptor (CAR) directed against CD19 elicit high response rates in relapsed or refractory (r/r) B-cell nonHodgkin lymphoma (B-NHL). However, r/r B-NHL remissions are durable in fewer than half of recipients of second-generation (2G) CAR T-cells. Third-generation (3G) CARs employ two co-stimulatory domains, resulting in improved CAR T-cell efficacy in vitro and in animal models in vivo. This investigator-initiated, phase 1 dose escalation trial, termed ENABLE, will investigate the safety and preliminary efficacy of WZTL-002, comprising autologous T-cells expressing a 3G anti-CD19 CAR incorporating the intracellular signalling domains of CD28 and Toll like receptor 2 (TLR2) for the treatment of r/r B-NHL.
\end{abstract}

Methods and analysis: Eligible participants will be adults with $\mathrm{r} / \mathrm{r}$ B-NHL including diffuse large B-cell lymphoma and its variants, follicular lymphoma, transformed follicular lymphoma and mantle cell lymphoma. Participants must have satisfactory organ function, and lack other curative options. Autologous T-cells will be obtained by leukapheresis. Following WZTL-002 manufacture and product release, participants will receive lymphodepleting chemotherapy comprising intravenous fludarabine and cyclophosphamide. A single dose of WZTL-002 will be administered intravenously two days later. Targeted assessments for cytokine release syndrome (CRS) and immune cell effector-associated neurotoxicity syndrome (ICANS), graded by ASTCT criteria, will be made. A modified $3+3$ dose escalation scheme is planned starting at $5 \times 10^{4} \mathrm{CAR}$ T-cells $/ \mathrm{kg}$ with a maximum dose of $1 \times 10^{6} \mathrm{CAR}$ T-cells $/ \mathrm{kg}$. The primary outcome of this trial is safety of WZTL-002.

Secondary outcomes include feasibility of WZTL-002 manufacture and preliminary measures of efficacy.

Ethics and dissemination: Ethical approval for the study was granted by the New Zealand Health and Disability Ethics Committee (reference 19/STH/69) on $23^{\text {rd }}$ June 2019 for 
Protocol Version 1.2. Trial results will be reported in a peer-reviewed journal, and results presented at scientific conferences or meetings.

Trial registration number: NCT04049513

Trial opened to recruitment on $30^{\text {th }}$ September 2019.

\subsection{Introduction}

\subsubsection{CAR T-cell therapy for B-cell non-Hodgkin lymphoma}

Non-Hodgkin lymphoma (NHL) is the 7th most common malignancy worldwide, accounting for over 200,000 deaths annually ${ }^{19}$. Over $90 \%$ of NHLs stem from the B-cell lineage (BNHL), and can be divided into aggressive and indolent forms ${ }^{20}$. While aggressive subtypes of B-NHL, exemplified by diffuse large B-cell lymphoma (DLBCL), are often cured with chemoimmunotherapy, around 20\% are either refractory to treatment or will relapse 2929730 . For most indolent B-NHL subtypes, such as follicular lymphoma (FL), relapses after chemoimmunotherapy are the norm, and while allogeneic stem cell transplantation is curative for some patients, its use is limited by significant short- and long-term toxicities, and by the need to identify a matched haematopoietic stem cell donor.

Autologous T-cells transduced to express a chimeric antigen receptor (CAR) specific for the B-cell antigen CD19 can lyse B-NHL cells ${ }^{298}$. Two such 'CAR T-cell therapies' have been licensed, incorporating a single intracellular co-stimulatory domain derived from either CD28 (axicabtagene ciloleucel) or 4-1BB (tisagenlecleucel). CAR T-cell therapies lead to impressive response rates in those with relapsed or refractory $(r / r) D_{\text {LBCL }}{ }^{1176}$, and with indolent B-NHL subtypes ${ }^{83}$. However, only $35-40 \%$ of recipients of currently-licensed CAR T-cells for DLBCL remain free of progression for longer than 12 months, a lack of complete metabolic response by six months being a major predictor of CAR T-cell treatment failure ${ }^{78}$. Anti-CD19 CAR T-cell therapies that exhibit improved early complete metabolic response rates and long-term disease-free survival rates could fulfil an unmet need in r/r BNHL 11299 .

\subsubsection{Third-generation CAR T-cells}

One way of enhancing CAR T-cell efficacy is to incorporate a second intracellular costimulatory domain within the CAR, generating so-called 'third-generation' (3G) CAR Tcells ${ }^{200}$. This can lead to improved CAR T-cell proliferation, cytotoxicity and persistence in 
vivo ${ }^{300,190}$. Most 3G CAR T-cells in registered clinical trials combine a co-stimulatory domain derived from an immunoglobulin (Ig) superfamily member (such as CD28 or ICOS) alongside one derived from a tumour necrosis factor receptor (TNFR) superfamily member (such as 41BB or OX40), see Table $1^{200}$. Potential benefits of $3 \mathrm{G}$ CAR constructs over $2 \mathrm{G}$ CARs have been demonstrated in pre-clinical studies ${ }^{17185301}$. For example, Zhao et al. reported that $3 \mathrm{G}$ CARs containing both $\mathrm{CD} 28$ and $41 \mathrm{BB}$ costimulatory domains, led to greater expansion of $\mathrm{CD}^{+}$and $\mathrm{CD} 8^{+} \mathrm{T}$-cells, along with improved B-ALL tumour regression in xenograft models ${ }^{185}$. However, it is not yet clear whether 3G CAR T-cells offer improved clinical efficacy.

Activated T-cells express Toll-like receptors (TLRs), particularly TLR2, a pattern recognition receptor that recognizes bacterial cell wall components ${ }^{302} 303$. Ligation of TLR2 enhances Akt and Erk1/Erk2 phosphorylation in response to T-cell receptor (TCR) stimulation, enhancing TCR-induced cytokine production and proliferation ${ }^{189}$. T-cell intrinsic TLR2 signalling lowers the T-cell activation threshold in response to costimulatory signals received from antigen presenting cells, and enables the generation of functional memory CD8 T-cells in response to T-cell activation 304305 .

Third generation CAR T-cells incorporating the Toll/interleukin-1 receptor (TIR) domain from TLR2, which mediates the intracellular signalling of TLR2, show improved anti-tumor activity compared to second-generation (2G) CAR T-cells both in vitro and in vivo ${ }^{17}$. The safety and efficacy of a 3G CAR T-cell product combining CD28 and TLR2 TIR costimulatory domains has been explored in a Phase I clinical trial in B-cell acute lymphoblastic leukaemia (B-ALL) (ClinicalTrials.gov reference NCT02822326), in which clinical responses were observed, including among participants with extra-medullary B-ALL tumours ${ }^{18}$.

We have modified the manufacture of $3 \mathrm{G}$ anti-CD19 CAR T-cells incorporating CD28 and TLR2 TIR co-stimulatory domains, to employ a third-generation self-inactivating lentiviral vector for T-cell transduction and to adopt process modifications designed to meet local Good Manufacturing Practice (GMP) requirements. We plan a phase 1 dose escalation trial to assess the safety of this product, WZTL-002, for the treatment of r/r B-NHL. 
Box 5.1 Strengths and Limitations of this Study

\section{Strengths and limitations of this study}

Uses a new third-generation (3G) anti-CD19 chimeric antigen receptor (CAR) construct incorporating both CD28 and Toll-like receptor 2 (TLR2) costimulatory domains.

- Establishes feasibility of T-cell harvest, CAR Tcell manufacture and treatment delivery at a New Zealand centre.

- Employs consensus grading systems for cytokine release syndrome and immune cell effectorassociated neurotoxicity syndrome.

- Dose escalation and dosing steps similar to those employed in other $3 \mathrm{G}$ anti-CD19 CAR T-cell trials.

- Small sample size, inclusion of several B-cell nonHodgkin's lymphoma subtypes and dose escalation design means that efficacy and exploratory outcomes will be descriptive only.

\subsection{Methods}

\subsubsection{Study design}

This investigator-initiated open-label phase 1 dose escalation trial is named ENABLE:

Engaging Toll-like Receptor Signalling for B-cell Lymphoma Chimeric Antigen Receptor Therapy, (ClinicalTrial.gov number: NCT04049513). The ENABLE trial aims to assess the safety of WZTL-002, comprising autologous anti-CD19 3G CAR-T cells incorporating CD28 and TLR2 TIR co-stimulatory domains, for the treatment of $r / r$ B-NHL. The sponsor is the Malaghan Institute of Medical Research (MIMR), and the trial is conducted in collaboration with Wellington Zhaotai Therapies Limited (WZTL). The Study Site is Wellington Hospital, Capital \& Coast District Health Board, New Zealand.

Key inclusion and exclusion criteria are presented in Box 5.2. In addition to the inclusion and exclusion criteria presented in Box 5.2, immunosuppressive therapies, with the exception of lymphodepleting chemotherapy, must be avoided during the week before WZTL-002 administration (72 hours for systemic corticosteroids). Prior autologous and allogeneic stem cell recipients are eligible to participate in the Study. Structure of the CAR employed in WZTL-002 is presented in Figure 5.1. 
A modified $3+3$ dose escalation scheme with four dose steps $\left(5 \times 10^{4} ; 1 \times 10^{5} ; 5 \times 10^{5} ; 1 \times\right.$ $10^{6} \mathrm{CAR}$ T-cells $\left./ \mathrm{kg}\right)$ is planned. The first dose step is two steps (10-fold) below the recommended phase II dose determined in a phase I trial of a similar product in $\mathrm{r} / \mathrm{r}$ B-ALL (ClinicalTrials.gov reference NCT02822326), and is similar to that used in two reported clinical trials of 3 G CAR T-cell products ${ }^{190}{ }^{192}$. The final dose step was selected because dose-limiting toxicities (DLTs) were observed at this level in $r / r$ B-ALL using a similar product (ClinicalTrials.gov reference NCT02822326) and because, based on preclinical data, the dose of WZTL-002 is expected to be lower than that recommended for the licensed $2 \mathrm{G}$ CAR T-cell product axicabtagene ciloleucel $\left(2 \times 10^{6} \mathrm{CAR} \mathrm{T}\right.$-cells $\left./ \mathrm{kg}\right)$. Additional dose steps may be incorporated if recommended by the Data Safety Monitoring Committee (DSMC). The DLT definitions are presented in Box 5.3 Dose Limiting Toxicities. The modified ' $3+3$ ' dose escalation study design is shown in Figure 5.2.

Figure 5.1 Diagrammatic representation of WZTL-002 Anti-CD19 third generation CAR Tcell illustrating the co-stimulatory domains and components of the chimeric antigen receptor

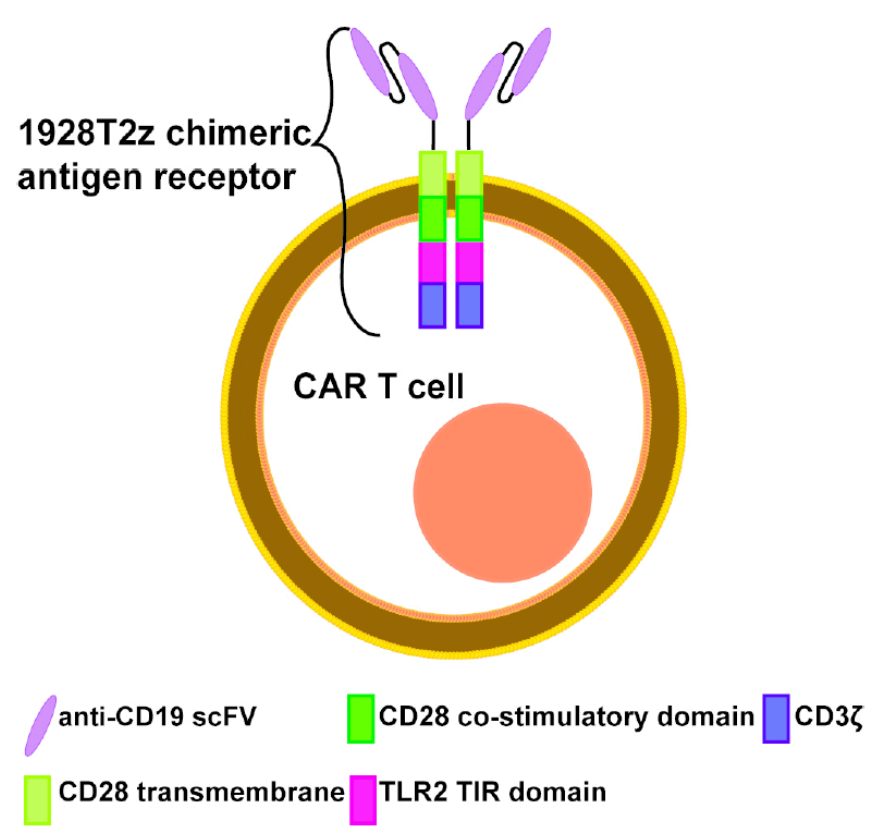


Figure 5.2 Dose escalation ENABLE Study Design using ' $3+3$ ' Design.

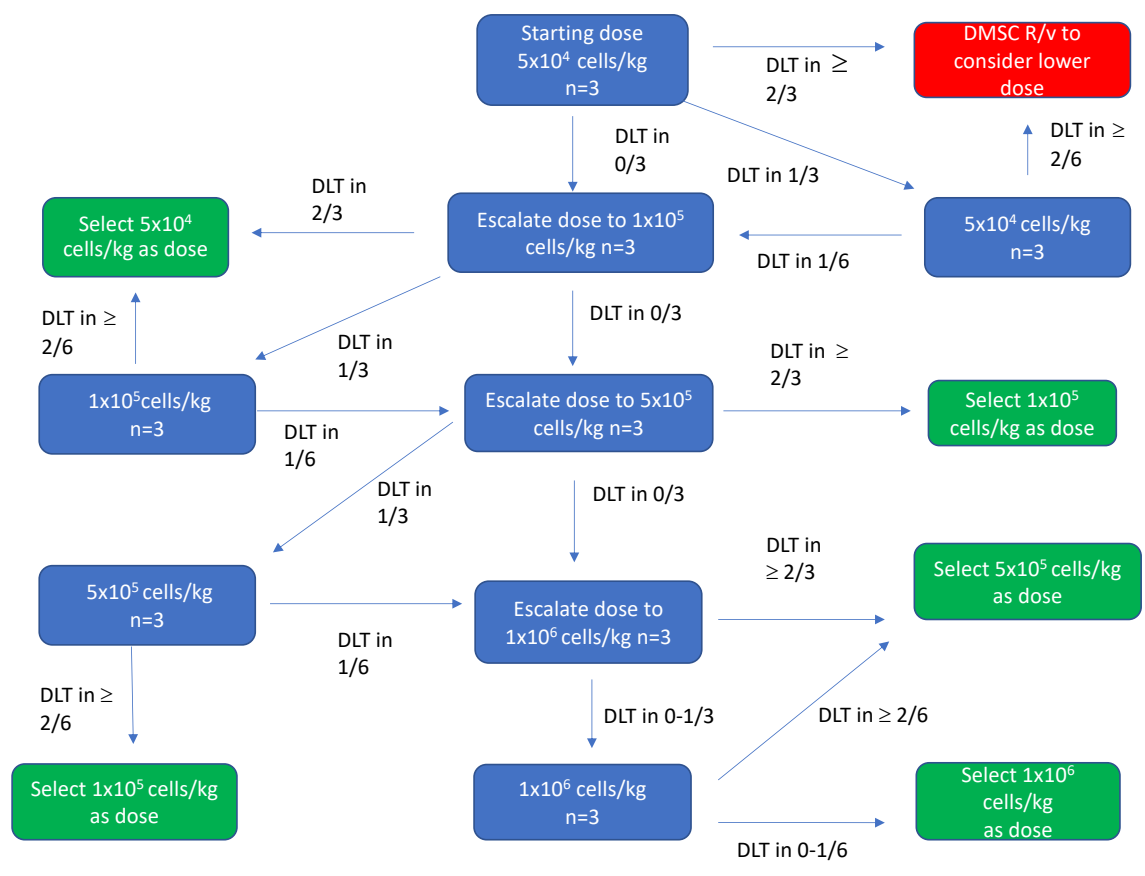




\section{Box 5.2 Inclusion and Exclusion Criteria for the ENABLE Trial}

\section{Inclusion criteria}

- Age 16-75 years (inclusive).

- Biopsy-proven relapsed or treatment refractory B-cell non-Hodgkin's lymphoma of the following subtypes per WHO classification: diffuse large B-cell lymphoma (DLBCL) and its variants, primary mediastinal B-cell lymphoma (PMBCL), transformed follicular lymphoma (tFL), follicular lymphoma (FL) and mantle cell lymphoma (MCL).

- Requirement for treatment in the opinion of the investigator

- No other curative treatments available, or not suitable due to patient or disease characteristics or lack of stem cell donor.

- Malignancy documented to express CD19 based on flow cytometric or immunohistochemical staining.

- Provision of written informed consent for this study.

- Life expectancy from non-Iymphoma-related causes of >12 months.

- European Cooperative Oncology Group (ECOG) performance status of 0 to 2 inclusive.

- Adequate haematologic function, defined by neutrophils $\geq 1.0 \times 10^{9} / \mathrm{L}$ and platelets $\geq 50 \times 10^{9} / \mathrm{L}$.

- No serious cardiac, pulmonary, hepatic or renal disease.

- Serum bilirubin $<2.5$ times upper limit of normal (ULN).

- Estimated creatinine clearance $\geq 50 \mathrm{~mL} / \mathrm{min}$ using the modified Cockroft Gault estimation or as assessed by direct measurement.

- Cardiac ejection fraction $\geq 50 \%$ as determined by echocardiogram or MUGA scan.

- Oxygen saturations $>92 \%$ on room air.

- Diffuse capacity of the lungs for carbon monoxide (DLCO) or carbon monoxide transfer coefficient (KCO), forced expiratory volume in one second (FEV1) and forced vital capacity (FVC) are all $\geq 50 \%$ of predicted by spirometry after correcting for haemoglobin and/or volume on lung function testing.

\section{Exclusion criteria}

- Confirmed active or prior central nervous system (CNS) involvement by lymphoma. In patients with a clinical suspicion of CNS disease, lumbar puncture and MRI brain must be performed.

- Active CNS pathology including: epilepsy, seizure within the preceding year, aphasia, paresis, stroke, dementia, psychosis within the preceding year, severe brain injury, Parkinson disease or cerebellar disease.

Richter syndrome.

- Active autoimmune disease requiring systemic immunosuppression.

- Prior solid organ transplantation.

- Allogeneic stem cell transplantation within the preceding 3 months or still requiring systemic immunosuppression.

- Current grade II-IV acute graft vs host disease (GVHD), any prior grade IV acute GVHD, or current moderate or severe chronic GVHD.

- Need for systemic corticosteroids to treat a condition other than B$\mathrm{NHL}$ at a daily dose of $\geq 10 \mathrm{mg}$ prednisone (or equivalent).

- Peripheral blood lymphocytes $<0.5 \times 10^{9} / \mathrm{L}$ as assessed by complete blood count.

- Peripheral blood $\mathrm{CD}^{+}$T-cells $<350 / \mu \mathrm{L}$ as assessed by lymphocyte subset analysis.

- Pregnant or lactating female.

- Women of childbearing potential who are not willing to use highly effective methods of contraception during study participation and for at least 1 year after WZTL-002 administration.

- Men who are not willing to use highly effective methods of contraception during study participation and for at least 1 year after WZTL002 administration.

- Men who have a pregnant partner and are not willing to use a condom while performing sexual activity during study participation and for at least 3 months after WZTL-002 administration.

- Subjects with known sensitivity to immunoglobulin or to components of the investigational product (IP).

- History of active malignancy other than B-cell malignancy within 2 years prior to enrolment, with the exception of: adequately treated in situ carcinoma of the cervix; adequately treated basal cell carcinoma (BCC) or localised squamous cell carcinoma (SCC) of the skin; other localised malignancy surgically resected (or radically treated with another treatment modality) with curative intent.

- Current or prior HIV infection.

Vaccination with a live virus within the preceding 4 weeks.

- Treatment with a purine analogue within the preceding 4 weeks.

- Treatment with alemtuzumab within the preceding 12 weeks.

- Prior gene therapy, including prior anti-CD19 chimeric antigen receptor T-cell therapy.

- Receipt of an investigational medicine within another clinical trial within the preceding 4 weeks.

- Inadequately controlled systemic infection.

- Serologic status reflecting active viral hepatitis B or any history of hepatitis $C$ infection as follows:

- Presence of hepatitis B surface antigen $(\mathrm{HBsAg})$ or hepatitis B core antibody $(\mathrm{HBCAb})$. Patients with presence of $\mathrm{HBcAb}$, but absence of $\mathrm{HBsAg}$, are eligible if hepatitis $B$ virus (HBV) DNA is undetectable $(<20 \mid \mathrm{U})$, and if they are willing to receive appropriate anti-viral prophylaxis.

- Presence of hepatitis $\mathrm{C}$ virus (HCV) antibody.

- Presence of New York Heart Association (NYHA) class 2 or higher cardiac symptoms not related to lymphoma.

- Significant concomitant illnesses which would in the investigator's opinion make the patient an unsuitable candidate for the trial.

- Subjects who have diminished capacity or any circumstance that would prohibit them from understanding and providing informed consent in accordance with ICH-GCP (International Conference on Harmonisation, Good Clinical Practice).

Subject does not provide consent to enrol onto International Cellular Therapy Registry. 


\subsubsection{Study procedures}

All potential participants will be assessed at a lymphoma multidisciplinary team meeting to confirm that no other curative treatment options are available. Following written informed consent and screening, eligible participants will undergo a leukapheresis procedure to harvest autologous peripheral blood mononuclear cells for WZTL-002 manufacture. Following cell harvest, bridging therapy will be permitted to provide disease control during manufacturing and treatment scheduling, and to reduce lymphoma disease bulk before WZTL-002 administration. Anti-microbial prophylaxis and tumour lysis prophylaxis will be given as per standard of care for patients receiving treatment for haematological malignancies. Once product release criteria are met, eligibility to proceed to WZTL-002 treatment is confirmed, and following any bridging chemo- or radiotherapy, a baseline PET/CT scan will be performed. This will be followed by lymphodepleting chemotherapy comprising intravenous fludarabine $\left(30 \mathrm{mg} / \mathrm{m}^{2} /\right.$ day $\times 3$ days $)$ and cyclophosphamide $\left(500 \mathrm{mg} / \mathrm{m}^{2} /\right.$ day $\times 3$ days). WZTL-002 will be administered following two chemotherapy-free days as a slow intravenous push. Participants will be monitored as an inpatient for 14 days, using both regular observations and specific cytokine release syndrome (CRS) and neurotoxicity assessments, including the Immune Effector-cell Encephalopathy (ICE) score, at least twice daily ${ }^{149}$. Daily assessment will continue until 21 days after WZTL-002 administration. To inform treatment of the next participant, assessment of DLTs will be undertaken 21 days after WZTL-002 administration (see Box 5.3 Dose Limiting Toxicities).

Response assessment will be by PET/CT scan three months after WZTL-002 administration using the Deauville 5-point scoring system, and response to treatment will be assigned as either Complete Response (CR), Partial Response (PR), Stable Disease (SD) or Progressive Disease (PD), according to 2014 Lugano response criteria for lymphoma ${ }^{306}$. A further PET/CT scan will be performed at 6 months for those with partial response at the 3 month timepoint. Additional imaging to assess or confirm treatment response, to investigate toxicities, or to seek potential disease progression, may be carried out at any time, as clinically indicated. Trial follow-up will take place at 3 monthly intervals until 1 year, 6 monthly intervals until 2 years and annually until 5 years post-WZTL-002 administration. 
Participants will be registered in the Center for International Blood and Marrow Transplant Research (CIBMTR) Cellular Therapies Registry and Australasian Bone Marrow Transplant Recipient Registry (ABMTRR), in order to capture low-incidence or late treatment-related toxicities. The protocol schema is shown in Figure 5.3 Schema for the enable phase I dose escalation study. Second attempt at cell harvest and WZTL-002 production may be considered at discretion of TMC. Six-month PET scan if first PET scan post WZTL-002 treatment shows partial response..

\section{Box 5.3 Dose Limiting Toxicities}

A Dose Limiting Toxicity (DLT) is a toxicity or Adverse Event (AE) occurring during the DLT assessment period (first 21 days after WZTL-002 administration), which is not attributable to a cause unrelated to WZTL002 (such as underlying lymphoma, concurrent illness or concomitant medications), and meets one of the following criteria:

- Grade 4 or greater CRS or ICANS or grade 3 CRS or ICANS that does not resolve to grade 2 or lower within 7 days, both as per the American Society for Transplantation and Cellular Therapy (ASTCT) criteria.

- Any adverse event requiring airway intubation (including neurotoxicity requiring intubation for airway protection).

- Grade 4 neutropenia that does not resolve to grade 3 or lower within 21 days after WZTL-002 administration.

- Platelet transfusion-dependent thrombocytopenia persisting for 21 days or longer after WZTL-002 administration.

- All grade 4 toxicities and grade 3 toxicities that do not resolve to grade 2 or lower within 7 days, with the exception of the following, which are not automatically considered DLTs:

- Myelosuppression, including

- Neutropenia.

- Bacterial infection in the setting of neutropenia with neutrophils $<1.0 \times 10^{9} / \mathrm{L}$.

- Thrombocytopenia.

- Bleeding in the setting of thrombocytopenia with platelets $<50 \times 10^{9} / \mathrm{L}$.

- Anaemia.

- Lymphopenia.

- Hypersensitivity reactions occurring within 2 hours of WZTL-002 administration (and considered related to cell administration) that resolve to grade 2 or less within 24 hours.

- Asymptomatic biochemical abnormalities that resolve to grade 2 or lower within 7 days.

- Hypogammaglobulinaemia.

For CRS and ICANS, ASTCT grading criteria will be used. For all other toxicities, CTCAE V.5.0 will be used.

AEs, adverse events; CRS, cytokine release syndrome; ICANS, immune cell effector-associated neurotoxicity syndrome. 
Figure 5.3 Schema for the enable phase I dose escalation study. Second attempt at cell harvest and WZTL-002 production may be considered at discretion of TMC. Six-month PET scan if first PET scan post WZTL-002 treatment shows partial response.

Long-term follow-up through bone marrow transplant clinic and Cellular Therapies Registry enrolment. FluCy, fludarabine and cyclophosphamide IV; PET, positron emission tomography; TMC, trial management committee.

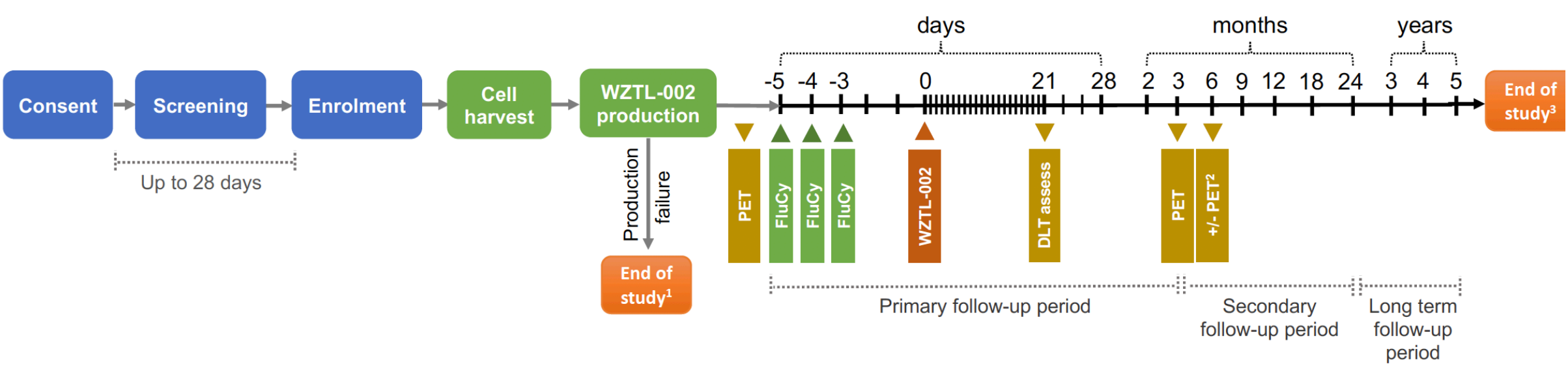

Notes and abbreviations:

1 Second attempt at cell harvest and WZTL-002 production may be considered at discretion of TMC

26 month PET scan if first PET scan post WZTL-002 treatment shows partial response

${ }^{3}$ Long-term follow-up through bone marrow transplant clinic and Cellular Therapies Registry enrolment

FluCy, fludarabine and cyclophosphamide IV; PET, positron-emission tomography/computed tomography scan 


\subsubsection{Study Aims and Outcomes}

The overall aim of the ENABLE trial is to assess the safety of $3 \mathrm{G}$ autologous anti-CD19 CAR T-cells incorporating CD28 and TLR2 TIR co-stimulatory domains (WZTL-002) in individuals with $\mathrm{r} / \mathrm{r}$ B-NHL.

The primary outcome is safety profile of WZTL-002, determined by the number and severity of adverse events assessed by CTCAE v5.0, except for Cytokine Release Syndrome and Immune Effector Cell-Associated Neurotoxicity Syndrome, which will be assessed by American Society Transplantation and Cellular Therapy (ASTCT) consensus grading criteria 126.

Secondary outcomes are as follows:

1. Feasibility of WZTL-002 manufacture, as determined by the proportion of enrolled participants undergoing at least one study leukapheresis procedure that receive WZTL-002

2. Overall response rate (ORR) as determined by complete response (CR) plus partial response (PR) 3 months after WZTL-002 administration

3. Cumulative CR rate 6 months after WZTL-002 administration

4. Relapse-free survival (RFS) for participants treated with WZTL-002 over a period of 24 months after WZTL-002 administration

5. Overall survival (OS) for participants treated with WZTL-002 over a period of 24 months after WZTL-002 administration

6. The recommended phase 2 dose of WZTL-002 for the treatment of patients with $\mathrm{r} / \mathrm{r}$ B-NHL

Exploratory outcomes are:

1. Kinetics and persistence of WZTL-002 following administration, determined by peripheral blood PCR for the CAR transgene 
2. Extent and duration of B-cell aplasia, determined by peripheral blood flow cytometry and serum immunoglobulin $\mathrm{G}$ concentration

3. Serum cytokine profile following WZTL-002 administration

4. Phenotype of the WZTL-002 CAR T-cell product before administration, and of circulating CAR T-cells following administration.

\subsubsection{Manufacture of WZTL-002}

WZTL-002 will be manufactured in the Clinical Human Immunology Laboratory at the Malaghan Institute of Medical Research in Wellington, New Zealand, which is licensed by Medsafe, the New Zealand Medicines and Medical Devices Safety Authority. Briefly, peripheral blood mononuclear cells (PBMCs) are isolated from the leukapheresis using a density gradient medium. T-cells are selected and activated using immunomagnetic CD3/CD28 microbeads and genetically modified using a third-generation generation selfinactivating non-replication competent lentiviral vector (manufactured in-house and tested according to EMEA guidelines). After washing to remove lentiviral vector and microbeads, CAR T-cells are expanded in a GMP-grade medium supplemented with IL-2 and human serum for 7 days. The CAR T-cell product is harvested and formulated in a cryopreservation medium containing 10\% DMSO. Release criteria for the CAR T-cell product include product sterility, identity, purity and absence of residual lentiviral vector.

\subsubsection{Immune monitoring and exploratory endpoints}

Kinetics of WZTL-002 will be determined by determining the CAR transgene in patient peripheral blood by quantitative PCR before and at 1, 2, 4, 7, 9, 11, 14, 16, 18, 21 and 28 days, at 2, 3, 6, 12 and 24 months, and at 3, 4 and 5 years, after WZTL-002 administration. Serum cytokine profile after WZTL-002 administration will be determined by ELISA (for IL-6) and by cytokine bead array before and at $1,2,4,7,9,11,14,16,18,21$ and 28 days after WZTL-002 administration. At each timepoint $25 \mathrm{~mL}$ of blood is taken for study-specific analyses. In subjects with neurotoxicity, CSF samples may be stored for assessment of cytokine and CAR transgene levels. Depth and duration of B-cell aplasia will be established by peripheral blood flow cytometry and by nephelometric determination of serum immunoglobulin $\mathrm{G}$ concentration, both to inform infection risk among participants, and to serve as a 
surrogate measure for WZTL-002 persistence. The immunophenotype (including CD4, CD8, CD45RA and CD62L) of WZTL-002 CAR T-cells will be determined in the pre-administration product, and within participant peripheral blood mononuclear cells after administration (if CAR T-cells are detectable).

Samples of the CAR T-cell product and recipient PBMCs post-administration (at each protocolised timepoint) will be cryopreserved. The timepoints for exploratory analyses will be selected based on pharmacokinetics determined by PCR for the CAR transgene. The outcomes of exploratory analyses are not expected to be definitive, and are included to inform design of a subsequent efficacy trial.

\subsubsection{Toxicity Management}

Based on clinical experience with similar constructs, CRS and ICANS are anticipated toxicities of WZTL-002. Accordingly, a CAR T-cell toxicity (CARTOX) team comprising local intensive care, neurology, haematology, immunology and infectious disease specialists and nursing representatives was formed. This team localised consensus assessment and treatment protocols for CRS and ICANS, and reviewed safety measures and training and competency assessment materials ${ }^{149,307}$. A summary of key measures taken to prepare for CRS and ICANS is provided in

Box 5.4. 
Box 5.4 Summary of measures taken to prepare for CRS and ICANS 
- Formation of CAR T-cell toxicity working group composed of haematologists, neurologist, intensivist, immunologist and haematology nurses.

- Attendance of principal investigator, coinvestigators, study nurse, senior haematology nurses and nurse educators at a CAR T-cell toxicities preceptorship day.

- Localisation of guidelines for CRS and ICANS identification, management and escalation, and the upload of these to institutional electronic treatment guide.

- Education sessions and competency assessments on CAR T-cell toxicities delivered to nursing, patient at risk and ICU teams.

- Allowance for bridging treatment between leukapheresis and lymphodepleting chemotherapy to 'debulk' or control disease before WZTL-002 administration.

- Confirmation that three doses of tocilizumab are on site before WZTL-002 administration.

- Levetiracetam $750 \mathrm{mg}$ twice daily to be given for 28 days following WZTL-002 administration for anti-seizure prophylaxis.

- Completion of clinical checklist before WZTL-002 administration.

- Notification of neurology and intensive care unit teams before WZTL-002 administration.

- Scheduled nurse-led CRS and ICANS assessments.

- Provision of participant-held wallet card and discharge summary sheet.

CRS, cytokine release syndrome; ICANS, immune cell effector-associated neurotoxicity syndrome.

\subsubsection{Monitoring and data management}

A trial management committee (TMC) including the Principal Investigator, at least one CoInvestigator, the study nurse, and a representative of the Clinical Human Immunology Laboratory, will meet at least monthly during study recruitment to review recruitment rates, trial conduct, trial procedures, Adverse Events (AEs) and Serious Adverse Events (SAEs). An independent Data and Safety Monitoring Committee (DSMC) will include clinicians with experience in early phase T-cell trials and in haemato-oncology. Per the DSMC Charter, the DSMC will meet and review trial accrual, conduct and safety data a minimum of 6-monthly 
and before each dose step. An independent study monitor will monitor the study, and will report to the Sponsor.

The Study site will hold responsibility for the confidentiality of electronic and paper clinical records held for the study participant. To maintain confidentiality of trial participants, study data or samples sent to collaborating investigators or external contractors for analysis or review will be labelled with study-specific codes, and not with patient identifiers. The Principal Investigator will hold responsibility for ensuring that presentations and publications of the study findings do not contain identifiable information. Laboratory records will be kept for a minimum of fifteen years. Clinical data (including Case Report Forms) will be stored securely for a minimum of fifteen years.

\subsubsection{Statistical analysis}

This phase I trial will be analysed using descriptive statistics; no formal hypothesis testing will be undertaken. All participants who commence lymphodepleting chemotherapy will be included in the summaries of the safety outcomes.

Safety outcomes including AEs, SAEs, Suspected Unexpected Serious Adverse Reactions (SUSARs), CRS, ICANS and DLTs will be individually listed by dose group and summarised as the frequency of events and percentages of individuals experiencing each event type. Each event summary will include details of the timing, grade, and outcome of the event. Response outcomes including ORR (defined as CR rate plus PR rate) and CR rate, will be individually listed and summarised as frequencies (\%) by dose group. The survival outcomes, RFS and OS will also be summarised as frequencies (\%) at 24 months and the times to events will be individually listed and may be summarised with Kaplan-Meier curves for individual dose groups if sample sizes permit. Associations between safety outcomes and presenting features will be explored in a qualitative manner.

The study sample size will depend upon dose-limiting toxicities observed during dose escalation, and is estimated at 12 participants, with at least 3 participants treated at each dose step. If no DLTs are observed, escalation to the next dose step may occur (see Box 2 for DLT definition). If a DLT is observed in 1 of the first 3 participants treated at a specific dose step, a further 3 participants should be treated at that dose. If 2 or more participants develops a DLT 
at a specific dose step, escalation to the next dose step should not occur, indicating that the Maximum Tolerated Dose (MTD) has been reached. The DMSC will be meet before each proposed dose escalation and may recommend de-escalation to a lower dose level, protocol modification, or for more participants to be treated at that dose step, based on available safety and/or efficacy data.

\subsubsection{Ethics}

The study will performed in accordance with the principles of the International Conference on Harmonisation Guidelines on Good Clinical Practise (ICH-GCP) (Step 4, dated $10^{\text {th }}$ June 1996) that have their origins in the Declaration of Helsinki ${ }^{308}$.

The trial has been approved by the New Zealand Health and Disability Ethics Committee (reference 19/STH/69), and has been endorsed by Research Advisory Group Māori at Capital $\&$ Coast District Health Board, which is mandated to provide consultation for cultural appropriateness of clinical research conducted within the region (reference RAG-M \#662).

\subsubsection{Patient and Public Involvement}

The study protocol was developed after discussion at a blood cancer patient forum convened by Leukaemia \& Blood Cancer New Zealand, and at meetings of the Lymphoma Network of New Zealand and the NZ Branch of the Haematology Society of Australia and New Zealand. The study protocol and consent form were developed in consultation with Research Advisory Group - Māori, a Māori relationship board, which includes lay representation, to Capital \& Coast District Health Board. The participant information and consent form was reviewed by a patient representative with relevant personal experience. The study has been publicised in national media, although due to regulatory and logistical considerations, referrals must come from a relevant specialist rather than directly from potential participants. Study results will be presented in the lay media as well as in scientific journals. The patient information and consent form includes an option to request a lay summary of the study results. 


\subsubsection{Data Dissemination}

Participants will be given the option to receive a summary of the trial results. Trial results will be published in a peer-reviewed journal after completion of the trial.

\subsection{Discussion}

This manuscript describes the protocol for ENABLE, an investigator-led Phase 1 dose escalation trial evaluating a new third generation (3G) autologous anti-CD19 CAR T-cell product (WZTL-002), for the treatment of individuals with $\mathrm{r} / \mathrm{r}$ B-NHL. The primary outcome is safety, which will be assessed by determining the number and severity of adverse events. Secondary outcomes will assess feasibility, efficacy and recommended WZTL-002 dose for subsequent efficacy trials.

As well as resulting in improved cytotoxicity against target cells, the incorporation of a second co-stimulatory domain can enhance CAR T-cell proliferation and cytokine production 17200 . Thus, while 3G CAR T-cell products have the potential for increased efficacy, there is also the potential for an increased risk of toxicities including CRS and ICANS risk, compared to second-generation products. Accordingly, CRS and ICANS were identified as events of special interest early during trial development. The risk of both toxicities may relate to CAR T-cell dose and to disease burden ${ }^{113} 114$. Therefore, to mitigate CRS and ICANS risks, conservative starting and maximum WZTL-002 doses have been selected, based on clinical experience using similar CAR T-cell products. Targeted CRS and ICANS assessments will be performed, and a comprehensive risk mitigation plan has been developed, including the development of institutional policies and protocols, documented staff training, intensive care escalation plans and on-site tocilizumab availability. Use of the ASTCT international consensus CRS and ICANS grading system will facilitate the comparison of toxicity rates with other anti-CD19 CAR T-cell trials conducted internationally ${ }^{149}$.

The use of 'bridging' chemo- and radiotherapy between enrolment and WZTL-002 administration is permitted. This will facilitate WZTL-002 treatment scheduling during dose escalation, and may allow reduction of disease burden before WZTL-002 therapy, potentially reducing CRS and ICANS risk ${ }^{71}$. To mitigate the impact of bridging therapy on efficacy 
assessments, baseline PET-CT scans will be conducted after completing bridging therapy and before starting lymphodepleting chemotherapy and WZTL-002 administration.

The selection of eligible lymphoma subtypes was based on evidence of efficacy from other clinical trials evaluating anti-CD19 CAR T-cell therapies in this population ${ }^{11} 76828386$. Limitations of this trial include its small size, inclusion of several B-NHL subtypes, and dose escalation design, as a result of which efficacy and exploratory outcomes will be descriptive only. In particular, secondary outcomes assessing WZTL-002 efficacy (ORR, CR rate, RFS and OS) will be preliminary, and are included to help inform the design of future Phase II trials. Similarly, the exploratory outcomes, which explore WZTL-002 kinetics, phenotype, serum cytokines and B-cell aplasia, are intended to inform outcome measure selection for future larger trials.

The published clinical experience of $3 \mathrm{G}$ anti-CD19 CAR T-cells for the treatment of $\mathrm{r} / \mathrm{r} \mathrm{B}-$ NHL is limited, with the final results of only two other early-phase trials published, to our knowledge $\mathrm{e}^{192} 234$. Enblad et al treated 11 patients with $\mathrm{r} / \mathrm{r}$ B-NHL or Chronic Lymphocytic Leukaemia (CLL) with 3G anti-CD19 CAR T-cells combining CD28 and 41BB costimulatory domains, in a Phase 1 dose escalation study ${ }^{192}$. Of the 11 treated participants, four did not receive lymphodepletion before CAR T-cell administration. The dose range of $3 \mathrm{G}$ anti-CD19 CAR T-cells administered this study was $2 \times 10^{7}-2 \times 10^{8}$ cells $/ \mathrm{m}^{2}$ (approximately equivalent to $5 \times 10^{5}-5 \times 10^{6} \mathrm{CAR}$ T-cells $/ \mathrm{kg}$ ). A response to treatment was observed in four participants (36\%), all of whom reached $\mathrm{CR}^{192}$. Severe CRS was reported in two participants (18\%), and severe neurotoxicity in one $(9 \%)$.

Ramos et al reported results of a Phase 1 anti-CD19 CAR T-cell trial involving simultaneous administration of autologous 2G (CD28 only) and 3G (4-1BB plus CD28) anti-CD19 CAR Tcell products to participants with r/r B-NHL ${ }^{234}$. This dose escalation study treated 11 participants with active lymphoma and five in remission after autologous stem cell transplant (ASTCT). All participants with active lymphoma received lymphodepletion with cyclophosphamide and fludarabine before CAR T-cell infusion, whereas no further lymphodepletion was given to those post ASTCT. The dose range of total CAR T-cells administered on this study ( $2 \mathrm{G}+3 \mathrm{G}$ CAR T-cells in $1: 1$ ratio), was $5 \times 10^{4}-1 \times 10^{6} \mathrm{CAR} \mathrm{T}$ cells/kg. Six of 11 with active lymphoma (54\%) responded, three (27\%) reaching CR. All five recipients of CAR T-cells after ASTCT remained in CR at least 9 months after CAR Tcell administration. No cases of severe CRS, and only one of severe neurotoxicity, were 
reported ${ }^{234}$. Ramos et al found that the $3 \mathrm{G}$ anti-CD19 CARs showed superior in vivo expansion and persisted longer than their $2 \mathrm{G}$ counterparts, although the relative contribution of the $2 \mathrm{G}$ and $3 \mathrm{G}$ CAR T-cells to anti-tumour efficacy and to toxicity could not be assessed with this study design ${ }^{234}$.

In conclusion, published phase I trials suggest that manufacture of 3G CAR T-cells is feasible, and do not yet indicate that CRS and ICANS rates are higher than for $2 \mathrm{G}$ products. Moreover, the Ramos et al study indicates that 3G CAR T-cells can exhibit improved proliferation and persistence in humans compared to $2 \mathrm{G}$ counterparts. However, because of the small number of reported 3G CAR T-cell recipients, and the likely suboptimal CAR Tcell dosing in the early cohorts of these dose escalation studies, conclusions cannot be drawn about the relative efficacy and safety of $3 \mathrm{G}$ compared with $2 \mathrm{G}$ CAR T-cells ${ }^{192}{ }^{234}$. Other $3 \mathrm{G}$ anti-CD19 CAR T-cell trials in patients with $\mathrm{r} / \mathrm{r}$ B-NHL are underway (see As well as adding to the clinical experience of 3G anti-CD19 CAR T-cell therapies for the treatment of B-NHL, the ENABLE trial will inform the clinical safety and potential utility of a new intracellular TLR2 co-stimulatory domain within CAR T-cells 
Table 5.1 Other third generation anti-CD19 CAR T-cell trials registered on clinicaltrials.gov

\begin{tabular}{|c|c|c|c|c|c|c|}
\hline \\
\hline $\begin{array}{l}\text { Study } \\
\text { ClinicalTrials govID }\end{array}$ & $\begin{array}{l}\text { B-cell malignancy } \\
\text { subtypes }\end{array}$ & $\begin{array}{l}\text { CAR } \\
\text { generation }\end{array}$ & $\begin{array}{l}\text { Study } \\
\text { Phase }\end{array}$ & Lymphodepletion & Study Start Date & $\begin{array}{l}\text { Results published } \\
\text { (Yes/no) }\end{array}$ \\
\hline NCT02963038 & B-ALL + B-NHL & $3 G$ & $\mathrm{I}+\mathrm{II}$ & Not specified & June 2016 & No \\
\hline NCT03068416 & B-ALL + B-NHL & $3 \mathrm{G}$ & II & Not specified & September 2017 & No \\
\hline $\begin{array}{l}\text { NCT02132624 } \\
\text { (see discussion section } \\
\text { in paper) }\end{array}$ & B-NHL & $3 \mathrm{G}$ & I & Flu $25 \mathrm{mg} / \mathrm{m}^{2} \times 3 \mathrm{~d}$, Cy $500 \mathrm{mg} / \mathrm{m}^{2} \times 3 \mathrm{~d}$ & April 2014 & Yes $^{\dagger}$ \\
\hline NCT03146533 & B-NHL & $3 \mathrm{G}$ & $\mathrm{I}+\mathrm{II}$ & Flu $30 \mathrm{mg} / \mathrm{m}^{2} \times 3 \mathrm{~d}$, Cy $800 \mathrm{mg} / \mathrm{m}^{2} \times 3 \mathrm{~d}$ & April 2017 & No \\
\hline $\begin{array}{l}\text { NCT01853631 } \\
\text { (see discussion section } \\
\text { in paper) }\end{array}$ & B-ALL + B-NHL & $3 \mathrm{G}$ and $2 \mathrm{G}^{*}$ & I & Flu $30 \mathrm{mg} / \mathrm{m}^{2} \times 3 \mathrm{~d}$, Cy $500 \mathrm{mg} / \mathrm{m}^{2} \times 3 \mathrm{~d}$ & February 2014 & Yes $\ddagger^{\ddagger}$ \\
\hline NCT03676504 & B-ALL + B-NHL & $3 \mathrm{G}$ & I+II & Flu $30 \mathrm{mg} / \mathrm{m}^{2} \times 3 \mathrm{~d}$, Cy $500 \mathrm{mg} / \mathrm{m}^{2} \times 3 \mathrm{~d}$ & September 2018 & No \\
\hline NCT02822326 & B-ALL & $3 G$ & I & Flu $25 \mathrm{mg} / \mathrm{m}^{2} \times 3 \mathrm{~d}$ Cy $300 \mathrm{mg} / \mathrm{m}^{2} \times 3 \mathrm{~d}$ & January 2016 & No \\
\hline
\end{tabular}

As at $16 / 09 / 2019$

B-ALL, B-cell Acute Lymphoblastic Leukaemia; B-NHL, B-cell non-Hodgkin lymphoma

*Co-infused with CD28 containing second-generation CAR and CD28 + 41BB containing third generation

CAR

${ }^{\dagger}$ See Enblad et $\mathrm{al}^{24}$

${ }^{\dagger}$ See Ramos et $\mathrm{al}^{25}$ 


\section{Preliminary ENABLE trial experience}

The ENABLE Trial opened to recruitment for potential participants throughout New Zealand on October $1^{\text {st }} 2019$. At the time of writing, on $30^{\text {th }}$ April 2020, three participants have been enrolled onto the ENABLE trial, two participants have undergone leukapheresis and one participant has been treated with WZTL-002. On $13^{\text {th }}$ February 2020, the ENABLE Trial Management Committee (TMC) elected to halt recruitment until some manufacturing issues in the Good Manufacturing Practice (GMP) Laboratory at the MIMR (see Chapter Discussion), have been overcome.

\subsection{Referrals, pre-screening and screening experience}

As of $11^{\text {th }}$ May 2020, the list below outlines the current progress on the ENABLE trial in terms of pre-screening, screening, enrolment and study treatment.

- Trial enquiries: 15

- Ineligible pre-screening: 8

- Formal trial referrals: 4

- Possible future referrals: 3

- Participants screened: 4

- Participants enrolled: 3

- Participants undergone leukapheresis: 3

- Participants treated: 1 


\subsubsection{Pre-screening}

The reasons for patient enquiries not being eligible to proceed to screening on the ENABLE trial so far, are summarised in Table 6.1.

Table 6.1 Reasons for pre-screening ineligibility on the ENABLE Trial until April 2020

\begin{tabular}{|c|c|c|c|c|}
\hline Age & Sex & Diagnosis & Previous treatment & Reason not screened \\
\hline 30s & Female & $\begin{array}{l}\text { Grey Zone } \\
\text { Lymphoma }\end{array}$ & R-CHOP, R-ICE & $\begin{array}{l}\text { Atypical histology; } \\
\text { population of CD19- } \\
\text { malignant cells. }\end{array}$ \\
\hline $60 \mathrm{~s}$ & Female & $\begin{array}{l}\text { DLCBL relapsed } \\
\text { post Auto SCT }\end{array}$ & $\begin{array}{l}\text { R-CHOP + HD Mtx, } \\
\text { R-ICE, Auto SCT }\end{array}$ & $\begin{array}{l}\text { Severe } \\
\text { thrombocytopenia }\end{array}$ \\
\hline $60 s$ & Female & $\begin{array}{l}\text { Primary } \\
\text { refractory DH } \\
\text { lymphoma }\end{array}$ & $\begin{array}{l}\text { DA-EPOCH-R, } \\
\text { R-ICE }\end{array}$ & $\begin{array}{l}\text { Rapid deterioration } \\
\text { after enquiry; died }\end{array}$ \\
\hline $60 s$ & Male & $\begin{array}{l}\text { Relapsed DH } \\
\text { lymphoma }\end{array}$ & $\begin{array}{l}\text { DA-EPOCH-R, } \\
\text { R-ICE x } 4 \text { at relapse }\end{array}$ & $\begin{array}{l}\text { Severe lymphopenia } \\
\text { at time of enquiry } \\
\text { (lymphocytes } 0.1 \text { ) } \\
\text { AKI not meeting } \\
\text { inclusion criteria }\end{array}$ \\
\hline $60 \mathrm{~s}$ & Male & $\begin{array}{l}\text { Multiply relapsed } \\
\text { Follicular } \\
\text { Lymphoma }\end{array}$ & $\begin{array}{l}\text { PRIMA study, BEAM } \\
\text { Auto SCT 2015, ME- } \\
41 \text { trial }\end{array}$ & $\begin{array}{l}\text { CNS involvement } \\
\text { (neural foramina \& } \\
\text { CSF) }\end{array}$ \\
\hline $70 s$ & Male & $\begin{array}{l}\text { DLBCL, early } \\
\text { relapse at } 6 / 12 \\
\text { post CR }\end{array}$ & RCHOP x 6 & $\begin{array}{l}\text { Clinical deterioration, } \\
\text { not trial candidate }\end{array}$ \\
\hline 40s & Male & $\begin{array}{l}\text { Primary } \\
\text { refractory } \\
\text { DLCBL }\end{array}$ & RCHOP x 6 & $\begin{array}{l}\text { Not yet had salvage - } \\
\text { subsequent response } \\
\text { to R-ICE salvage and } \\
\text { planned to proceed to } \\
\text { Auto SCT }\end{array}$ \\
\hline $50 s$ & Male & $\begin{array}{l}\text { Primary } \\
\text { refractory } \\
\text { DLCBL }\end{array}$ & $\begin{array}{l}\text { RCHOP, R-ICE, R- } \\
\text { GDP }\end{array}$ & $\begin{array}{l}\text { Patient declined trial } \\
\text { referral }\end{array}$ \\
\hline
\end{tabular}

Abbreviations: RCHOP, Rituximab, Cyclophosphamide, Doxorubicin, Vincristine, Prednisone; HD Mtx, High dose Methotrexate; R-ICE, Rituximab, Ifosfamide, Carboplatin, Etoposide; DA-EPOCH-R, Dose adjuted Etoposide, Prednisone, Vincristine, Cyclophosphamide, Doxorubicin and Rituximab; BEAM, Carmustine, Etoposide, Cytarabine; R-GDP, Rituximab, Gemcitibine, Dexamethasone and Cisplatin; AKI, Acute Kidney Injury; CNS, Central nervous system; CSF, Cerebrospinal fluid.| 


\subsubsection{Screening}

A summary of the four participants screened on the ENABLE trial so far is provided in Table 6.2

Table 6.2 Screened participants in the ENABLE trial until April 2020

\begin{tabular}{|c|c|c|c|c|}
\hline Age & Sex & Diagnosis & Previous Treatment & $\begin{array}{l}\text { Eligible for } \\
\text { enrolment? }\end{array}$ \\
\hline $50 \mathrm{~s}$ & Male & $\begin{array}{l}\text { Primary } \\
\text { refractory } \\
\text { Follicular } \\
\text { Lymphoma }\end{array}$ & -RCHOP, R-ICE & Yes \\
\hline $50 \mathrm{~s}$ & Male & $\begin{array}{l}\text { Multiply relapsed } \\
\text { Mantle Cell } \\
\text { Lymphoma }\end{array}$ & $\begin{array}{l}\text {-RCHOP/ HD Ara-C } \\
\text {-BEAM Auto SCT } \\
\text {-R-ICE, Ibrutinib } \\
\text {-Flu/Mel/ATG MUD Allo- } \\
\text { SCT } \\
\text {-Rituximab + lenalidomide }\end{array}$ & Yes \\
\hline $60 \mathrm{~s}$ & Female & $\begin{array}{l}\text { Relapsed DH } \\
\text { Lymphoma }\end{array}$ & $\begin{array}{l}\text {-DA EPOCH-R } \\
\text {-R-GDP }\end{array}$ & Yes \\
\hline $60 \mathrm{~s}$ & Female & $\begin{array}{l}\text { Multiple relapsed } \\
\text { Follicular } \\
\text { Lymphoma }\end{array}$ & $\begin{array}{l}\text {-RCHOP } \\
\text {-R-ICE } \\
\text {-R-GDP } \\
\text {-Qbinutuzumab + } \\
\text { Idasanutlin + Venetoclax } \\
\text {-Radiotherapy }\end{array}$ & $\begin{array}{l}\text { Pending - after } \\
\text { excluding Alle } \\
\text { SCT as potential } \\
\text { curative option }\end{array}$ \\
\hline
\end{tabular}

Abbreviations: RCHOP, Rituximab, Cyclophosphamide, Doxorubicin, Vincristine, Prednisone; R-ICE, Rituximab, Ifosfamide, Carboplatin, Etoposide; HD Ara-C, High dose Cytarabine; Flu/Mel/ATG MUD Allo-SCT, Fludarabine, Melphalan, Anti-thymocyte globulin conditioned Matched Unrelated Donor Allogeneic Stem Cell Transplant; R-GDP, Rituximab, Gemcitibine, Dexamethasone and Cisplatin. 


\subsubsection{Enrolled Participants}

This section will briefly document the progress of the three currently enrolled participants until the time of WZTL-002 treatment (if applicable)

1. EN1-01:

- Following leukapheresis received 'bridging' radiotherapy and a pulse of rituximab + dexamethasone

- WZTL-002 product release criteria met (see Box 6.1)

- PET/CT Scan pre-lymphodepletion reported:

- Generalised reduction in size and FDG avidity of lymphadenopathy (Deauville 4). Increased avidity in neck nodes bilaterally (Deauville 5). Largest lymph node $48 \times 25 \mathrm{~mm}$ (mesenteric) in comparison to most recent $\mathrm{PET} / \mathrm{CT}$

- Fludarabine $30 \mathrm{mg} / \mathrm{m}^{2}$ and cyclophosphamide $500 \mathrm{mg} / \mathrm{m}^{2}$ daily on days -5 to -3 pre WZTL-002 administration

- WZTL-002 (dose of 5 x 104 anti-CD19 CAR T-cells/kg) administered

2. $\mathrm{EN1-02}$

- Initial leukapheresis procedure deferred due to low level hepatitis B DNA positivity detected

- MIMR GMP policies reviewed and altered to allow processing of samples from donors with low level hepatitis B DNA positivity, if ENABLE inclusion criteria met (see Box 5.2)

- Bridging therapy with bendamustine and obinutuzumab administered preleukapheresis

- Leukapheresis procedure carried out and WZTL-002 manufacture currently underway in MIMR GMP Laboratory 
3. EN1-03

- Following leukapheresis, R-GDP chemotherapy continued as bridging therapy

- WZTL-002 product did not meet required CAR T-cell dose after first GMP manufacturing run using fresh leukapheresis product due to poor in vitro CAR T-cell proliferation (see Chapter 7.5.2 Manufacturing protocol)

- WZTL-002 product did not meet required CAR T-cell dose after second GMP manufacturing run using cryopreserved Peripheral Blood Mononuclear Cells (PBMCs) from leukapheresis product due to poor in vitro CAR T-cell proliferation (see Chapter 7.5.2 Manufacturing protocol).

Box 6.1 WZTL-002 product characteristics for participant EN1-01

- WZTL-002 culture duration: 11 days

- Post thaw WTL-002 viability (\% of total cells) : $86 \%$

- Post thaw WZTL-002 frequency (\% live CAR T-cells out of total cells): $29 \%$

- Total number of viable WZTL-002 CAR T-cells produced : 14 x $10^{6}$ 


\subsection{Preliminary outcomes from Participant EN1-01}

As of $12^{\text {th }}$ February 2020, the primary follow up period for participant EN1-01 has been completed. This section will summarise the early safety and efficacy data available along with preliminary analyses from correlative studies.

\subsubsection{CRS and ICANS summary}

Data recorded in relation to CRS and ICANS following WZTL-002 treatment in participant EN1-01 is recorded in Table 6.3

A graph showing peak daily temperature and CRP during the primary follow up period for participant EN1-01, with CRS episodes and CRS treatment annotated, is shown in Figure 6.1.

Table 6.3 CRS and ICANS in participant EN1-01

\begin{tabular}{|l|l|l|l|l|}
\hline Participant & Assessment & $\begin{array}{l}\text { Highest Grade } \\
\text { (ATSCT) }\end{array}$ & Management & Outcome \\
\hline EN1-01 & CRS & Grade 1* & $\begin{array}{l}\text { Conservative } \\
\text { measures, } \\
\text { Tocilizumab IV } \\
\text { and } \\
\text { Dexamethasone } \\
\text { IV (second } \\
\text { episode) }\end{array}$ & Resolved \\
\cline { 2 - 5 } & ICANS & No ICANS & Not applicable & Not applicable \\
\hline
\end{tabular}

*First episode of CRS occurred 25 days after WZTL-002 administration

*Second episode of CRS occurred 46 days after WZTL-002 administration 
Figure 6.1 Graph showing temperature and CRP during EN1-01 primary follow up period
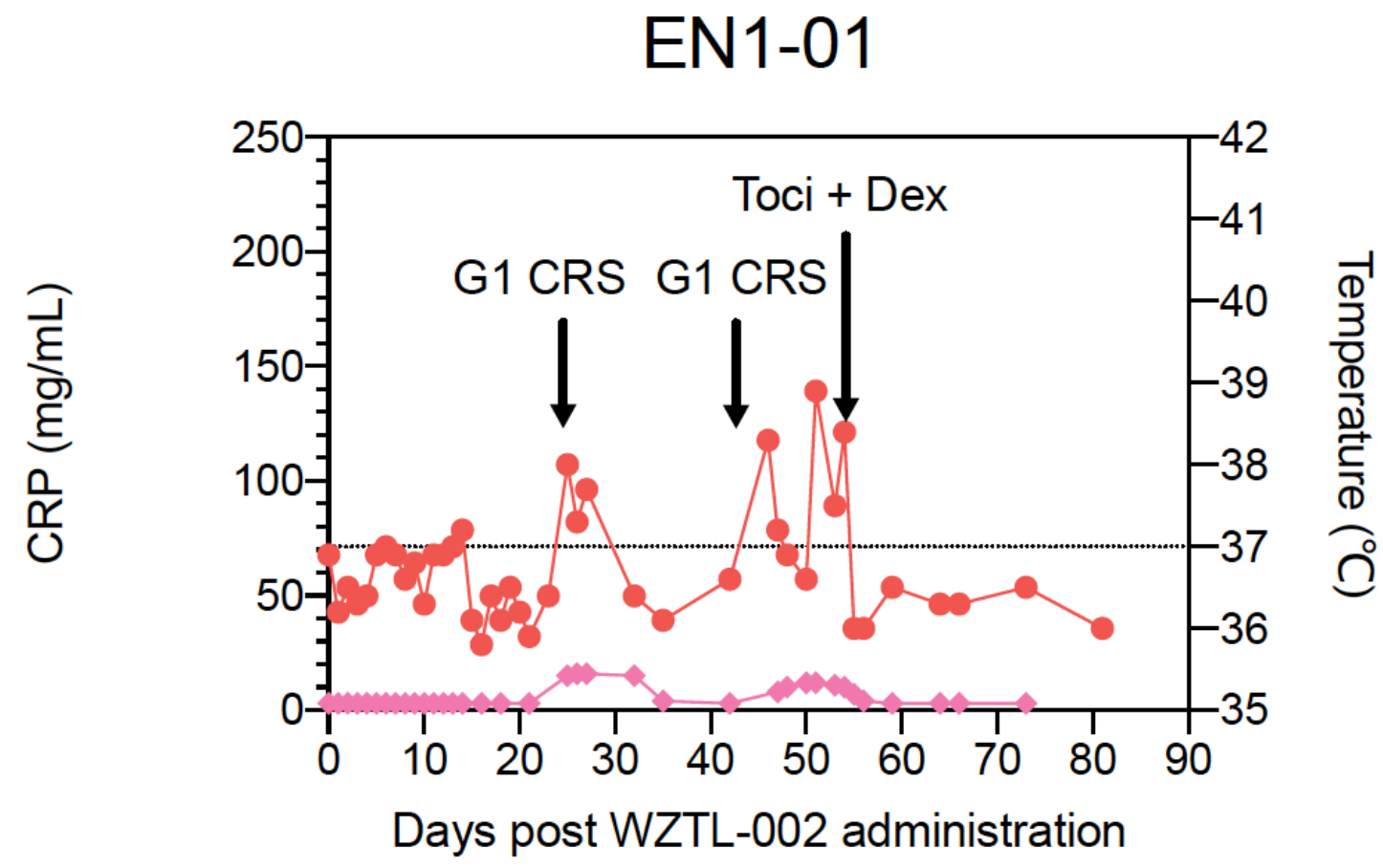

$\multimap-$ Temperature (highest) $\multimap$ CRP

Maximum temperature recordings (in ${ }^{\circ} \mathrm{C}$ as per right y axis) from the day of $W Z T L-002$ administration until 81 days post WZTL-002 administration are plotted as red dots. $C$ Reactive Protein (CRP) levels (in $\mathrm{mg} / \mathrm{mL}$ as per left y axis) recorded from the day of WZTL002 administration until 73 days post WZTL-002 administration, are plotted as pink dots.

\subsubsection{Adverse Event Summary}

A summary of all adverse events (AEs) and serious adverse events (SAEs) is provided in Table 6.4. 
Table 6.4 AE and SAE summary for EN1-01 during primary follow up period

\begin{tabular}{|c|c|c|c|c|c|c|}
\hline & & $\begin{array}{l}\text { CTCAE } \\
\text { v5.0 } \\
\text { (highest) }\end{array}$ & & & & \\
\hline \multirow{10}{*}{ EN1-01 } & Neutropenia & 4 & Resolved & Yes & No & No \\
\hline & Thrombocytopenia & 2 & Resolved & Yes & No & No \\
\hline & Lymphopenia & 3 & Resolved & Yes & No & No \\
\hline & $\begin{array}{l}\text { Cytokine Release } \\
\text { Criteria }\end{array}$ & $\begin{array}{l}1 \text { (ASCT } \\
\text { criteria) }\end{array}$ & Resolved & Yes & Yes & No \\
\hline & Hypernatraemia & 1 & Resolved & No & No & No \\
\hline & Hypoalbuminaemia & 1 & Resolved & Yes & No & No \\
\hline & Hyperphosphataemia & 1 & Resolved & Yes & No & No \\
\hline & Myalgia & 1 & Resolved & Yes & No & No \\
\hline & Maculopapular rash & 2 & Resolved & Yes & No & No \\
\hline & Mucositis & 2 & Resolved & Yes & No & No \\
\hline
\end{tabular}

\subsubsection{Efficacy summary}

A comparison was made between the PET/CT Scan pre lymphodepletion and the PET/CT scan carried out at 3 months post WZTL-002 treatment. De-identified images are shown in 
Figure 6.2, with a brief report underneath each image. Overall, the 3 month PET/CT assessment for participant EN1-01 represents disease progression of follicular lymphoma.

Figure 6.2 PET/CT Scan for EN1-01 before and after WZTL-002 treatment

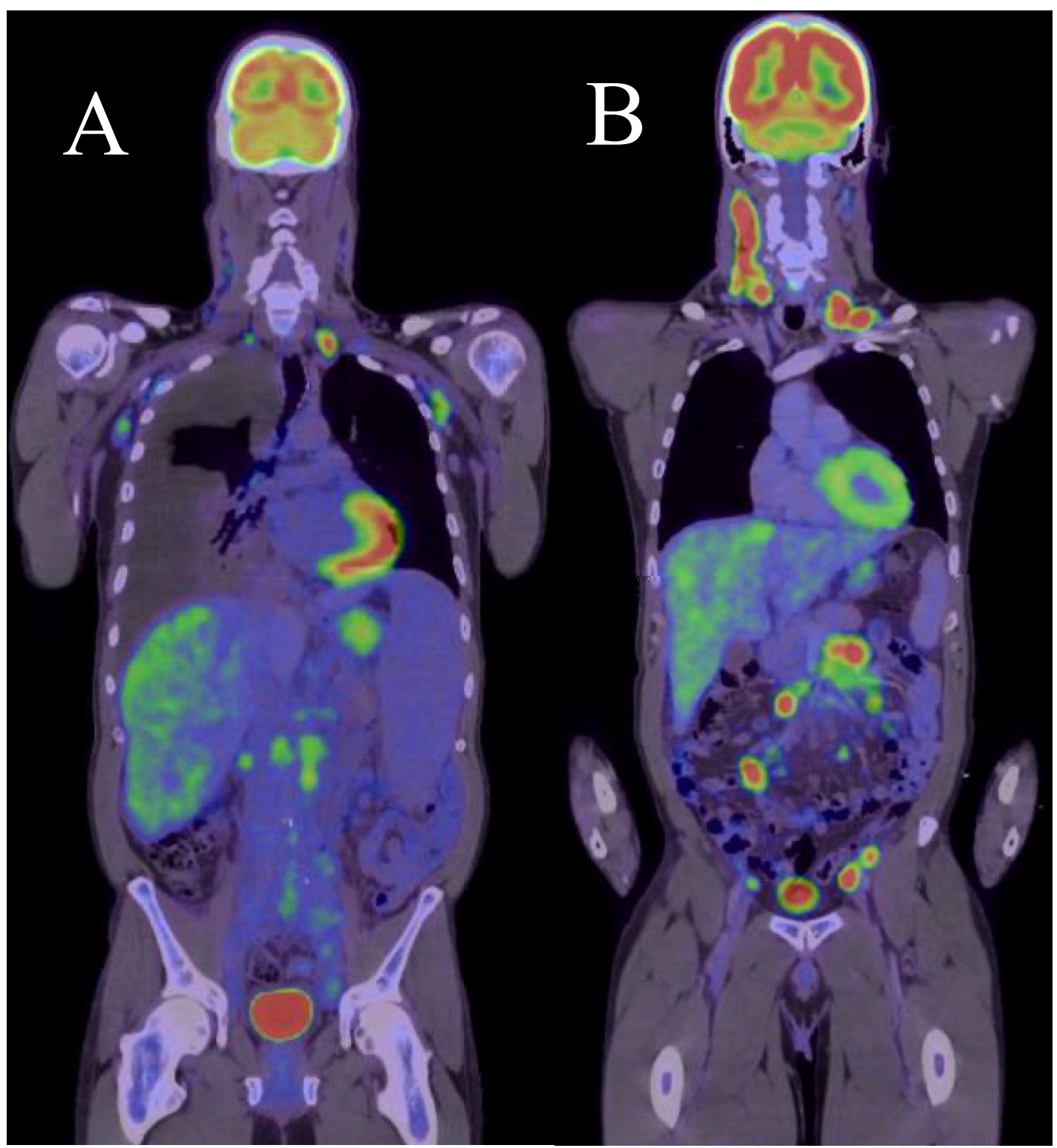

Deauville scale 5 Lymphadenopathy at the base of the neck. Deauville scale 4 lymphadenopathy within the abdomen and pelvis
Progression of disease in both CT and PET components in the neck and within the abdomen

Figure 6.2 (A) shows PET/CT image before lymphodepleting chemotherapy administered. 
(B) shows PET/CT at 3 months after WZTL-002 treatment.

\subsubsection{Correlative studies analysis}

Samples for correlative analysis were taken at specified time points throughout the primary follow up period as per the schedule of assessments in the ENABLE protocol (see Section 5.2.5 Immune monitoring and exploratory endpoints).

The WZTL-002 kinetics and IL-6 levels until day 21 following WZTL-002 administration are shown in Figure 6.3.

Figure 6.3 WZTL-002 kinetics and IL-6 levels until Day 21 post WZTL-002 administration

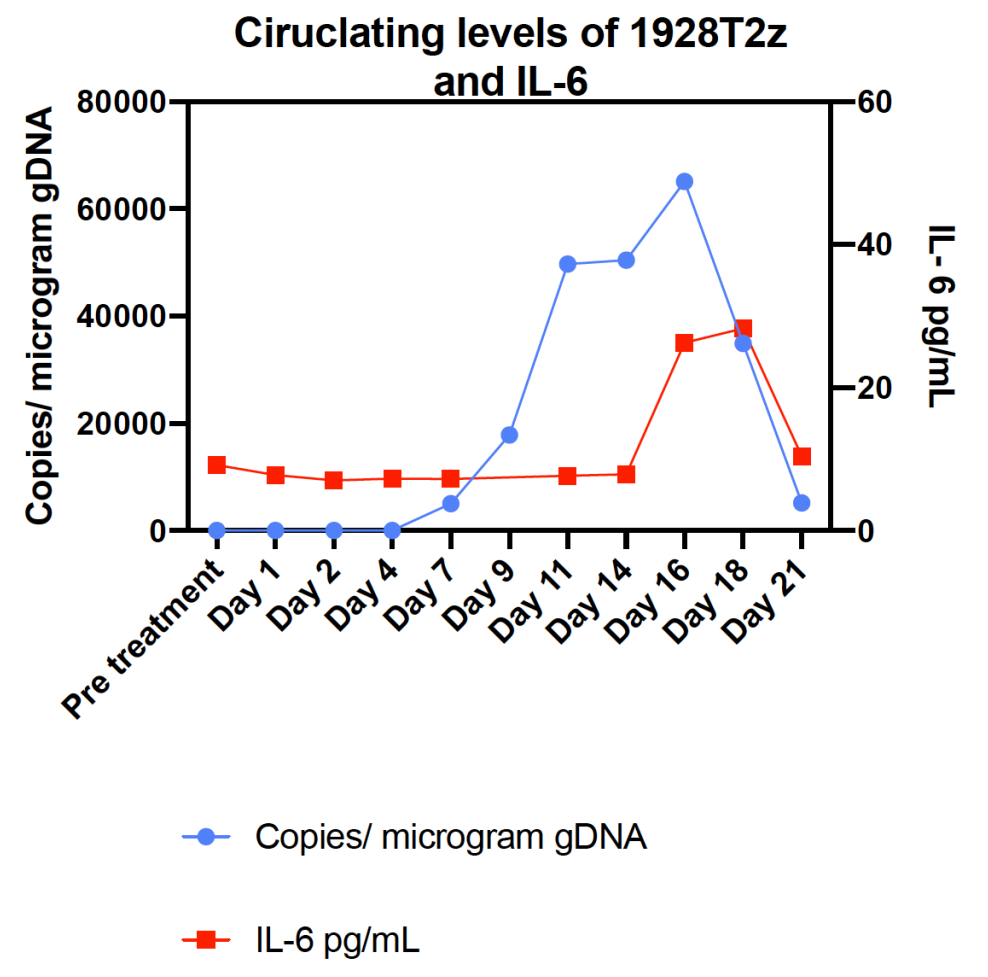

*Note Dr Nathaniel Dasyam performed the experiments and analysis to determine IL-6 and WZTL-002 kinetics. 
Following the development of delayed onset grade 1 CRS on day 46 post WZTL-002 administration, further analysis for IL-6 levels in serum samples taken between day 21 and day 51 of WZTL-002 infusion, was carried out (see Figure 6.4).

Figure 6.4 IL-6 levels until Day 51 post WZTL-002 administration

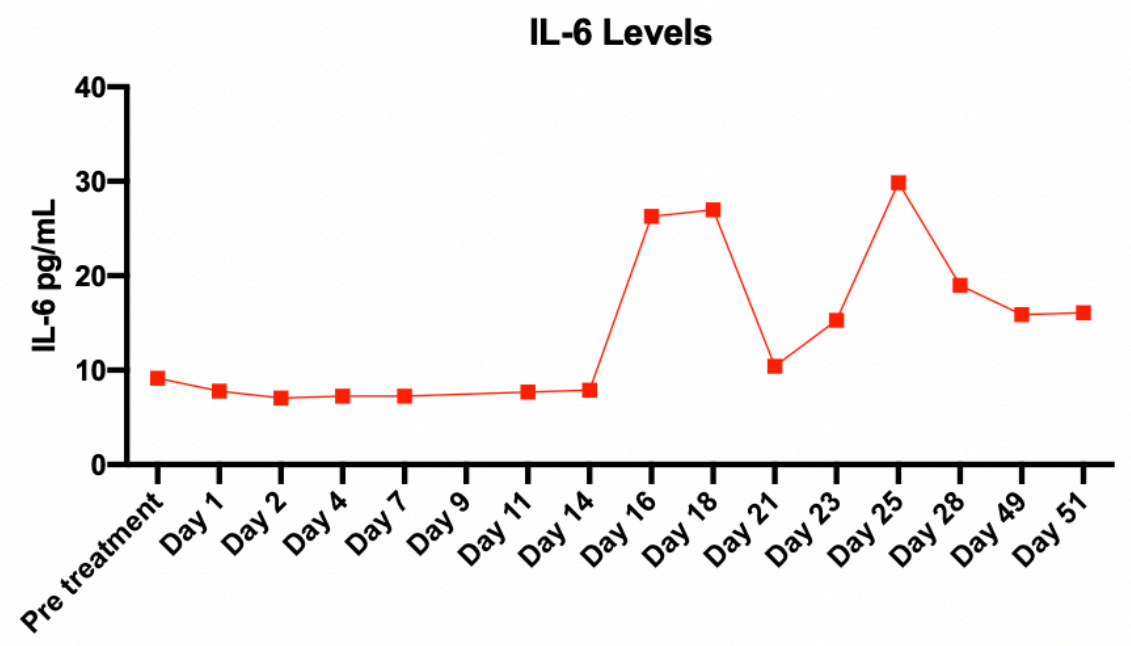

*Note Dr Nathaniel Dasyam performed the experiments and analysis to determine IL-6 levels.

\subsubsection{Conclusion}

In the primary follow up period for participant EN1-01, there were no DLTs identified. The ENABLE DSMC have reviewed the available clinical and correlative study data and have determined that the ENABLE trial can proceed to treat its second participant with WZTL-002 at the dose of $5 \times 10^{4} \mathrm{CAR}$ T-cells $/ \mathrm{kg}$ (first dose escalation step).

The safety profile was acceptable with two SAEs for two separate episodes of grade 1 CRS recorded. Tocilizumab and corticosteroids were administered due to refractory fevers associated with grade $1 \mathrm{CRS}$ and led to rapid resolution of CRS with normalisation of temperatures. 
Due to disease progression at 3 months, efficacy was not demonstrated for the first participant administered with WZTL-002. However, analysis of WZTL-002 kinetics by determining the CAR transgene in peripheral blood by quantitative PCR, showed that CAR T-cell expansion in vivo occurred, with peak WZTL-002 levels observed at day 16 reducing to very low levels at day 21 following WZTL-002 administration. Serum IL-6 levels were observed to peak at day 25 following WZTL-002 administration coinciding with the first episode of grade 1 CRS.

Overall, treatment of participant EN1-01 has demonstrated that anti-CD19 CAR T-cell therapy has been administered for the first time within New Zealand, highlighting useful information on CAR T-cell toxicity management and showing CAR T-cell expansion in vivo. 


\section{Discussion}

\subsection{Introduction}

This thesis was based on my experience working in a multidisciplinary team of scientists, clinicians, nurses, clinical trials specialists, project managers and technicians; to design, implement and commence New Zealand's first CAR T-cell trial.

The process of designing and setting up the ENABLE trial has involved liaising with a number of leading international experts within the cellular therapies field, which has informed several aspects of study design, risk mitigation strategies and clinical management protocols. In order to set up the ENABLE trial, a number of key regulatory approvals have been obtained and engagement with these regulators in New Zealand, will hopefully enable access to other CAR T-cell trials and/or commercial CAR T-cell treatments within New Zealand in the future. The main findings of this thesis are summarised below, followed by a discussion of some of the strengths and weaknesses of this study, then a discussion of the possible future direction of anti-CD19 CAR T-cell therapy and a concluding paragraph.

\subsection{Summary of main findings}

- Early and pro-active engagement with New Zealand regulatory bodies including but not limited to, Medsafe, the Environmental Protection Agency (EPA), the Gene Technology Advisory Committee (GTAC), the Health and Disability Ethics Committee (HDEC) and Research Advisory Group Māori (RAG-M), is crucial to ensure the safe and timely implementation of investigational CAR T-cell therapy within New Zealand (see Chapter 4. Chimeric antigen receptor T-cells in New Zealand: Challenges and Opportunities). I was the principal author of all the regulatory submissions for the trial detailed above. I constructed the applications for these regulatory submissions, attended the HDEC panel discussion for the ENABLE 
trial in person and carried out edits in response to the comments and feedback from the respective regulatory bodies.

- Utilisation of established infrastructure and policies within New Zealand, such as the New Zealand Blood Service leukapheresis process, is a key method to ensure CAR Tcell therapy is robustly and efficiently carried out within New Zealand (see Chapter 4. Chimeric antigen receptor T-cells in New Zealand: Challenges and Opportunities)

- Anti-CD19 CAR T-cell related toxicities are significant and potentially life threatening. Robust risk mitigation strategies, education and management protocols, are necessary to ensure the safe clinical delivery of CAR T-cell therapy (see Chapter 2.5 Anti-CD19 CAR T-cell therapy toxicities and Chapter 5.2.6 Toxicity Management)

- Optimal CAR T-cell dosing may need to take into account underlying tumour burden, recipient immune function, CAR T-cell product characteristics, CAR T-cell pharmacokinetic data and efficacy data; in order to mitigate toxicity and find a minimally effective dose rather than a maximum tolerated dose (see Chapter 3. CAR T-cell therapies: Optimising the dose). The chosen CAR T-cell dosing for the ENABLE trial is comparable to dosing from other third generation CAR T-cell trials (see Chapter 5.2.1 Study design).

- CAR T-cell manufacturing under Good Manufacturing Practice (GMP) conditions is an extremely complex process, with a vast number of permutations which may affect the feasibility of CAR T-cell manufacturing. Liaison with international collaborators carrying out investigational CAR T-cell trials is important to optimise this process (see Chapter 4. Chimeric antigen receptor T-cells in New Zealand: Challenges and Opportunities)

- Education and engagement with clinicians referring participants for anti-CD19 CAR T-cell therapy is crucial to maximise potential benefit for patients in terms of meeting trial eligibility criteria, maximising chances of successful T-cell harvest and potentially improving outcomes (see Chapter 5. Third-generation anti-CD19 chimeric antigen receptor $T$-cells incorporating a TLR2 domain for relapsed or refractory $B$ cell lymphoma: a phase I clinical trial protocol (ENABLE)) 


\subsection{Engagement with New Zealand regulatory bodies}

In order to introduce this new modality of cellular therapy into New Zealand, early engagement and maintaining a regular dialogue with a large number of regulatory bodies within New Zealand has been crucial.

In the ENABLE trial, the Investigational Medicinal Product (IMP), WZTL-002, is classified as a GMO under the HSNO Act 1996. The generation of GMOs is highly regulated by the New Zealand EPA under the HSNO Act, to ensure satisfactory risk management is in place to demonstrate negligible risk to the environment and public health. As the primary writer of the EPA application to release WZTL-002 from the PC2 containment laboratory at MIMR to the clinical trial site at Wellington Hospital; I found the following points were crucial to ensure a successful outcome:

- Early engagement with the EPA to review our application before official submission

- Regular contact with the EPA during the review process in order to address queries or concerns

- Clear documentation of extensive safety measures in place to ensure there is a negligible risk of CAR T-cells or lentiviral vector, to form a self-sustaining population and lead to adverse effects on public health and safety through inadvertent transmission.

Early engagement with both GTAC and RAG-M at Captial and Coast District Health Board (CCDHB), led to significant improvements to the ENABLE trial before it was commenced.

This early engagement, meant that a number of potential concerns had been addressed before we submitted these crucial regulatory applications and will hopefully ensure that CAR T-cell therapy within New Zealand is carried out more equitably and with more consideration of potential long term safety.

Key issues addressed by early engagement with GTAC, the committee appointed to approve trials involving gene therapies under the New Zealand Medicines Act 1981, are summarised as follows ${ }^{309}$ : 
1) Ensuring that all recipients of anti-CD19 CAR T-cell therapy are enrolled in an International Cellular Therapy Registry was a key recommendation from early consultation with the GTAC.

- This aligns the long term follow up of CAR T-cell recipients to the established method for monitoring the long term outcomes of bone marrow transplant recipients. Thereby potential low incidence or late onset toxicities following CAR T-cell therapy can be detected, providing a robust mechanism to ensure adequate long term safety data is obtained on this new modality of cellular therapy.

2) Ensuring that extensive consultation with clinicians from overseas with expertise in treating patients with CAR T-cell therapy, has taken place in order to optimise the management of CAR T-cell related toxicities.

- In order to address this, a DSMC which included members from the US and Australia with CAR T-cell treating experience and also clinicians within New Zealand who have extensive bone marrow transplantation experience, was assembled.

- The ENABLE clinical trial team attended a CAR T-cell preceptorship day at the Peter MacCallum Cancer Centre in Melbourne. This day gave us a comprehensive review of the training, toxicity monitoring and escalation processes at Australasia's leading CAR T-cell treating centre. Direct feedback of the written CRS and ICANS toxicity management guidelines for the ENABLE trial was received and this guidance and feedback was used to enhance educational material used for training clinical staff in Wellington Hospital on CAR T-cell related toxicities.

- I have had the opportunity to attend two international conferences where I received first hand advice on CAR T-cell toxicity management from international CAR T-cell experts (Neelapu and Turtle, personal communication). This advice has directly influenced CRS and ICANS management protocols adopted in Wellington Hospital.

Key issues addressed by early engagement with RAG-M at CCDHB are summarised as follows: 
1) Ensuring that information on CAR T-cell therapy provided to participants is provided in a clear, easy to understand format.

- The use of a plain English and te Reo summary along with a visual representation of CAR T-cell therapy (see Figure 4.1) was included in the Patient Information and Consent Form.

2) Consideration of measures to enhance equity of access to CAR T-cell therapy for patients from rural areas in New Zealand and lower socioeconomic groups.

- A memorandum of understanding was formed between MIMR and Leukaemia Blood and Cancer New Zealand (LBCNZ). This ensures that ENABLE trial participants who aren't eligible for travel and accommodation expenses reimbursement via the National Travel Assistance scheme, would receive funding for these costs from LBCNZ, thereby enhancing the equity of access to CAR T-cell therapy, including for Māori participants.

- Consultation with a Māori researcher and haematologist has provided useful insights into ensuring Māori engagement will continue to be addressed in the ENABLE trial.

\subsection{Strengths and limitations of study design}

The ENABLE trial was initially intended to be a Phase $1 \mathrm{~b}$ Study using data obtained from the Phase 1 dose escalation trial in Guangzhou for 1928zT2 CAR T-cells in patients with r/r BALL to inform the chosen dose for this trial.

However, as preparations for the ENABLE trial developed, it became increasingly apparent that there were a number of important differences between the IMP being tested in this trial (WZTL-002) and 1928zT2 cells that were tested in the Guangzhou Phase 1 dose escalation trial (see Chapter 1. Introduction). After a recommendation from a cellular therapies expert based in the US, who now serves as a member of the ENABLE DSMC (Professor Bollard, personal communication), it was agreed that given the significant differences between these two products, the ENABLE trial should be carried out as a Phase 1 dose escalation trial.

The ' $3+3$ ' dose escalation trial design adopted in the ENABLE trial, is a design that has been adopted in many phase 1 CAR T-cell trials to date 105192252310 . Dose escalation occurs after 
every three participants are treated until dose limiting toxicity is reached or a pre-specified maximum dose is met. As discussed in Chapter 3. CAR T-cell therapies: Optimising the dose, this study design may identify a higher dose than is needed for maximum clinical efficacy, thereby potentially increasing the risk of severe toxicities and reducing the manufacturing feasibility for the chosen dose. Instead, a clinical trial design that identifies a minimally effective dose (see Figure 3.3) that takes into account efficacy rates or CAR T-cell pharmacokinetics as well as toxicity, may be preferable for a Phase 1 CAR T-cell trial (see Section 3.6.4.2 ' $3+3$ ' dose escalation trial design).

A search carried out clinicaltrials.gov identifying CAR T-cell dosing information in antiCD19 CAR T-cell trials, found that the typical starting dose in CAR T-cell trials is within a total of $10^{6}$ to $10^{10} \mathrm{CAR}$ T-cells, and the difference between starting dose and MTD covering up to a $2 \log$ (100 fold) difference (see Figure 3.5). The ENABLE trial starts with a dose at the lower end of this range, with a total of $3.75 \times 10^{6}$ cells as the starting dose for a $75 \mathrm{~kg}$ participant (weight used in Figure 3.5 for total dose calculation). Given that WZTL-002 is a third generation CAR T-cell product incorporating the TLR2 costimulatory domain in addition to CD28 and has the potential for increased potency in comparison to its second generation CD28 containing counterpart, it is rational for the starting dose of WZTL-002 to be at the lower end of CAR T-cell trial doses adopted to date ${ }^{17}$. Furthermore the starting dose of the ENABLE trial is comparable to the chosen starting dose in other Phase 1 third generation CAR T-cell trials 190192261.

Another limitation to the ' $3+3$ ' dose escalation trial design, is the interpatient variability seen in CAR T-cell products and their pharmacokinetics ${ }^{86}$. Given the narrow therapeutic window seen in many CAR T-cell trials, a specified MTD identified through a standard ' $3+3$ ' dose escalation study, may not be adequate for certain patients ${ }^{133}$. For example, there may be low CAR T-cell expansion in a patient with minimal disease burden, leading to an identified MTD being sub therapeutic for that particular patient ${ }^{133268}$. This limitation could potentially be overcome using intra-patient dose escalation schemes, whereby an individual patient is given increasing doses of CAR T-cells based on toxicity and efficacy observed in that patient. Intra-patient dose escalation designs have been adopted in some anti-CD19 CAR T-cell trials currently recruiting patients (eg clinicaltrials.gov reference NCT02893189 and ANZCTR number ACTRN12617001579381). However, this study design has inherent limitations, such as the potential for reduced CAR T-cell expansion when subsequent doses are administered 
due to immunologic responses against the CAR, and the need to re-administer lymphodepleting chemotherapy before each dose of CAR T-cells to maximise the chance that CAR T-cell proliferation will occur9 825104 .

I worked closely with the study PI (Dr Robert Weinkove, Clinical Director of the Malaghan Institute) to develop the Study Design. The final Study Design evolved after several iterations following discussions both Dr Weinkove and I had with other clinical researchers, the Study statistician and International experts in Cellular Immunotherapies, along with extensive reading and research into the Study design of other early phase CAR T-cell trials.

Overall, in this study, the most established study design in Phase 1 CAR T-cell trials has been adopted in order to identify the MTD for WZTL-002 which may inform the chosen dose for a future dose expansion cohort or ultimately a Phase 2 study.

\subsection{Protocol modifications based on early trial experience}

\subsubsection{Clinical trial protocol}

Based on early experience gained from WZTL-002 manufacturing, patient enquiries and referrals from clinicians in New Zealand, carrying out study procedures under ICH-GCP and interactions with the DSMC, the independent study monitor and reviewers of the protocol manuscript published in BMJ Open; a number of protocol amendments were made and subsequently received approval by the Southern HDEC on $19^{\text {th }}$ February 2020.

A summary of the key protocol amendments and their rationale based on early experience from the ENABLE trial is provided in Table 7.1.

Table 7.1 ENABLE protocol amendments and rationale 


\begin{tabular}{|c|c|}
\hline $\begin{array}{l}\text { Widening inclusion criteria to } \\
\text { include Marginal Zone } \\
\text { Lymphoma as an eligible } \\
\text { histology for the trial }\end{array}$ & $\begin{array}{l}\text { Marginal Zone Lymphoma is a rare form of indolent b-cell } \\
\text { non-hodgkin lymphoma with few viable treatment options in } \\
\text { patients with relapsed or treatment refractory disease. There } \\
\text { have been increasing reports of efficacy with anti-CD19 CAR } \\
\text { T-cell therapy in the treatment of marginal zone lymphoma } \\
\text { 311. }\end{array}$ \\
\hline $\begin{array}{l}\text { Reduce total lymphocyte count } \\
\text { threshold to }<0.3 \times 10^{9} / 1 \text { and } \\
\text { total } \mathrm{Cd}^{+} \mathrm{T} \text {-cell count threshold } \\
\text { to }<150 / \mu 1 \text { in the exclusion } \\
\text { criteria }\end{array}$ & $\begin{array}{l}\text { The enable trial investigators have found that a reduced } \\
\text { lymphocyte count below the exclusion threshold of } 0.5 \times 10^{9} / 1 \\
\text { is relatively common in our targeted patient population, which } \\
\text { prevents some potential participants from being referred for } \\
\text { consideration of trial therapy. } \\
\text { A recent report suggested there is no clear correlation between } \\
\text { low pre-collection lymphocyte count (range } 0.19-0.49 \times 10^{9} / 1 \text { ) } \\
\text { and lymphocyte collection efficiency post apheresis }{ }^{248} \text {. } \\
\text { Experience from the GMP team at MIMR has determined that } \\
\text { the number of autologous T-cells collected from the } \\
\text { leukapheresis procedure significantly exceeds the numbers of } \\
\text { T-cells required for WZTL-002 manufacture. The reduced } \\
\text { lymphocyte and T-cell count thresholds in the exclusion } \\
\text { criteria aligns with criteria used in the phase II Juliet study }{ }^{76} \text {. }\end{array}$ \\
\hline $\begin{array}{l}\text { Increase time allowed between } \\
\text { Study enrolment and WZTL- } 002 \\
\text { administration from } 12 \text { weeks to } \\
24 \text { weeks }\end{array}$ & $\begin{array}{l}\text { Our experience with enrolled subjects, and discussion with } \\
\text { other early-phase trial clinicians indicates that delays between } \\
\text { subject enrolment, leukapheresis and CAR T-cell treatment } \\
\text { are likely in this patient group. Reasons include adverse } \\
\text { events (infection, disease progression, toxicity of bridging } \\
\text { regimen) before WZTL-002 treatment and scheduling } \\
\text { constraints (for manufacturing or clinical reasons, or due to } \\
\text { the constraints of the dose escalation schedule). } \\
\text { Therefore, we think it is appropriate to extend the allowed } \\
\text { time between study enrolment between study enrolment and } \\
\text { WZTL- } 002 \text { administration from } 12 \text { to } 24 \text { weeks. }\end{array}$ \\
\hline $\begin{array}{l}\text { Clarification that institutional } \\
\text { guidelines for CRS and ICANS } \\
\text { will be used for the management } \\
\text { these CAR T-cell related } \\
\text { toxicities. }\end{array}$ & $\begin{array}{l}\text { This clarification recognises that the CRS and ICANS } \\
\text { management algorithms should be frequently reviewed based } \\
\text { on emerging recommendations in this rapidly-developing field } \\
109 \text {, and according to recommendations of the cross- } \\
\text { disciplinary CAR T-cell toxicity (CARTOX) working group. }\end{array}$ \\
\hline $\begin{array}{l}\text { Clarification of Adverse Event } \\
\text { (AE) reporting after } 3 \text { months } \\
\text { post WZTL-002 administration } \\
\text { to ensure that only AEs related to } \\
\text { WZTL-002 treatment are } \\
\text { included. AEs that occur as a } \\
\text { result of progressive disease or } \\
\text { subsequent anti-lymphoma } \\
\text { therapies should be excluded. }\end{array}$ & $\begin{array}{l}\text { Based on data reported from Phase II anti-CD19 CAR T-cell } \\
\text { trials in patients with relapsed and refractory (r/r) B-cell } \\
\text { Lymphoma, it is likely that at least } 50 \% \text { of patients may have } \\
\text { disease progression within } 1 \text { year of anti-CD } 19 \text { CAR T-cell } \\
\text { therapy }{ }^{1176} \text {. Therefore, there may be a number of AEs that } \\
\text { occur as a result of disease progression and potentially after } \\
\text { further therapy for lymphoma are not directly related to } \\
\text { WZTL- } 002 \text { treatment. These changes were incorporated after } \\
\text { feedback from the ENABLE DSMC and Study Monitor. }\end{array}$ \\
\hline
\end{tabular}

\subsubsection{Manufacturing protocol}

The manufacture of the WZTL-002 product for EN1-01 met the GMP release criteria for treatment. However, it was noted that CAR T-cell proliferation during the culture period was 
significantly reduced in comparison to WZTL-002 products made from healthy donor validation manufacturing runs. Subsequently, there have been two unsuccessful WZTL-002 product manufacturing runs for participant EN1-03 due to inadequate CAR T-cell proliferation during the culture period, leading to insufficient numbers of CAR T-cells to make the $5 \times 10^{4} \mathrm{CAR}$ T-cells $/ \mathrm{kg}$ dose. The first GMP manufacturing run used fresh leukapheresis product and the second used cryopreserved PBMCs as starting material. After the second unsuccessful manufacturing run, the ENABLE TMC halted trial recruitment and the GMP CAR T-cell manufacturing team carried out experiments in the research laboratory to investigate the reasons for manufacturing failure. It should be noted that the principal secondary outcome of the ENABLE trial (see section 5.2.3 Study Aims and Outcomes), is the feasibility of WZTL-002 manufacture; hence optimising WZTL-002 product manufacturing is an important aim of the trial.

At this time it was discussed whether GMP validation manufacturing runs should have been conducted using PBMCs from patients with advanced lymphoma rather than healthy volunteers. The rationale for this being that immune cells manufactured from healthy donors have not been impacted by the immune effects of cancer or by the immunosuppressive effects of cytotoxic chemotherapy, rendering them very different to immune cells harvested from patients with advanced haematological malignancies ${ }^{250}$. Although using T-cells harvested from patients with advanced lymphoma for GMP validation manufacturing runs may provide more reliable information required for optimal CAR T-cell manufacture for trial participants, there are significant ethical considerations to make before recruiting a patient with advanced malignancy to go through an invasive procedure such as leukapheresis without any direct benefit to that individual. Overall, it was agreed that this did not meet GCP Principal 2: protocol which states 'research involving humans should be scientifically justified and described in a clear, detailed protocol' as it cannot be fully justified in accordance with the Nuremburg code which states: 'The experiment should be such as to yield fruitful results... unproducable by other methods or means of study, and not random and unnecessary in nature.'

A summary of some of the key steps taken to optimise the WZTL-002 product manufacturing protocol and their rationale, is outlined in Table 7.2. 
Table 7.2 Summary of key manufacturing changes taken to optimise WZTL-002 manufacturing

\begin{tabular}{|c|c|}
\hline Manufacturing Change(s) & Rationale \\
\hline $\begin{array}{l}\text { Direct liaison with NZBS to modify the } \\
\text { leukapheresis procedure for ENABLE } \\
\text { patients by: } \\
\text { 1) Increasing the blood volume } \\
\text { collected during the procedure to } \\
\text { two blood volumes (from one blood } \\
\text { volume originally) } \\
\text { 2) Altering the colour gradient selected } \\
\text { using the Spectra Optia }{ }^{\circledR} \text { Apheresis } \\
\text { System in order to collect less dense } \\
\text { cells }\end{array}$ & $\begin{array}{l}\text { In order to reduce red cell contamination } \\
\text { and improve the purity of PBMC collection } \\
\text { during leukapheresis without contamination } \\
\text { of other cells including red cells and other } \\
\text { granulocytes. } \\
\text { These changes were made in consultation } \\
\text { with NZBS clinicians and after consultation } \\
\text { with apheresis specialists from the Peter } \\
\text { MacCallum Cancer Centre, Melbourne. }\end{array}$ \\
\hline $\begin{array}{l}\text { Switching from fresh PBMCs to } \\
\text { cryopreserved PBMCs as starting material } \\
\text { for CAR T-cell manufacturing product. }\end{array}$ & $\begin{array}{l}\text { It is known that myeloid cells in peripheral } \\
\text { blood mononuclear cell concentrates inhibit } \\
\text { the expansion of CAR T-cells }{ }^{312} \text {. } \\
\text { Cryopreservation has been shown to reduce } \\
\text { the survival of myeloid derived suppressor } \\
\text { cells (MDSCs) and also to significantly } \\
\text { reduce their suppressive effect on T-cell } \\
\text { proliferation }{ }^{313} \text {. Therefore cryopreservation } \\
\text { of PBMCs obtained by leukaphereis may } \\
\text { improve CAR T-cell proliferation in ex vivo } \\
\text { culture. }\end{array}$ \\
\hline $\begin{array}{l}\text { Testing a variety of different conditions in } \\
\text { the CAR T-cell manufacturing process } \\
\text { including: day of lentiviral transduction of }\end{array}$ & $\begin{array}{l}\text { CAR T-cell manufacturing in the GMP } \\
\text { environment is very complex with a number } \\
\text { of factors to consider, therefore close liaison }\end{array}$ \\
\hline
\end{tabular}


T-cells, duration of dynabead addition, concentration of IL-2 and concentration of commercial AB serum used in T-cell culture period. with other CAR T-cell manufacturing centres is crucial ${ }^{203}$. Following discussion with other CAR T-cell researchers in Sydney and at the Fred Hutchinson Cancer Centre in Seattle, logical amendments to the WZTL-002 manufacturing process have been implemented.

It is yet to be fully determined exactly what modifications to the WZTL-002 manufacturing process will be successful to improve manufacturing feasibility on the ENABLE trial. Modifications and improvements to this complex part of investigational CAR T-cell therapy, will be an ongoing process as the trial progresses over the coming months and years.

\subsection{Significance of this trial}

The treatment of participant EN1-01 on the ENABLE trial was the first time that CAR T-cell therapy had been used as treatment for a patient within New Zealand. This is a significant milestone, as CAR T-cell therapy is a novel treatment modality which has altered the treatment paradigm of $\mathrm{r} / \mathrm{r}$ B-cell malignancies across the world over the past few years ${ }^{117687}$ 106128136 . Up until 2019, the only access to anti-CD19 CAR T-cell therapy for New Zealanders, has been through self-funding large amounts of money to receive this therapy abroad. For instance, David Downs spent close to $\$ 1,000,000$ in order to receive CAR T-cell therapy in Boston, US in January 2018; and has subsequently written a book called 'A Mild Touch of the Cancer,' where he describes his experience of cancer and of receiving CAR Tcell therapy ${ }^{314}$. He has now been in remission from Non-Hodgkin Lymphoma for over two years and is an advocate for the Malaghan Institute, committed to raising awareness and funds to support CAR T-cell therapy research at the Institute, though the 'Down with Cancer' campaign ${ }^{315}$. The process of establishing CAR T-cell manufacturing capability in New Zealand and setting up a Phase 1 clinical trial in New Zealand, will hopefully enable patients in similar circumstances to access this new treatment modality within New Zealand and without personal expense. Furthermore, it is hoped that the ENABLE trial will provide a 
platform for delivering CAR T-cell therapy within New Zealand in the future, whether through other phase 1 trials, larger phase 2 trials or through commercial CAR T-cell therapy.

The process of engaging with regulatory bodies within New Zealand and gaining multiple regulatory approvals to manufacture and administer CAR T-cells, has given these regulatory bodies an understanding into what CAR T-cell therapy involves, and provided a framework for what may be required to approve similar trials or commercial CAR T-cell therapy in the future. From a clinical perspective, the preparations made in Wellington Hospital for CAR Tcell therapy including producing electronic toxicity management guidelines, setting up a CAR T-cell toxicity working group, producing a CAR T-cell therapy nursing proforma and running competency-based assessments for clinical staff; have led to a robust clinical process to implement CAR T-cell therapy. In the process of setting up the ENABLE trial, a clinician led application was submitted to PHARMAC requesting funding for tocilizumab to treat CRS and ICANS on the ENABLE trial, and funding approval was granted in February $2019^{291}$. This is an important approval, as it demonstrates that a department within the New Zealand government have recognised the importance of tocilizumab in the management of these toxicities. These preparations will provide a solid foundation for CAR T-cell treatment at Wellington Hospital and will also be informative to clinicians from other centers planning to carry out CAR T-cell therapy from their treatment center within New Zealand, in the future.

The preliminary experience from the ENABLE trial to date has shown that a dose of WZTL002 can be manufactured in the MIMR GMP laboratory and that in vivo CAR T-cell expansion can be observed following administration. The safety data from participant EN101 was acceptable with no dose limiting toxicities reported, and the DSMC have agreed another participant can be treated on the trial to further evaluate the study objectives. Demonstrating that CAR T-cell manufacture can be carried out within New Zealand, could be particularly important in the context of the current global pandemic of severe acute respiratory syndrome coronavirus 2 (SARS-Cov-2), commonly termed COVID-19316. Recently published recommendations on the delivery of CAR T-cell therapy during the COVID-19 pandemic, highlight that the global pandemic may significantly complicate the logistics of delivering CAR T-cell therapy ${ }^{296}$. In particular, in order to access commercial CAR T-cell therapy, lymphocyte collection is usually carried out locally at the CAR T-cell treating center, then shipped to the manufacturer in the US for CAR T-cell production ${ }^{165}$. In the advent of COVID-19, there may be significant disruption to this process, meaning that 
CAR T-cell treating centers reliant on international shipping for obtaining cellular products, may be subject to significant delays or may be unable to deliver autologous CAR T-cell therapy at all for certain periods of time ${ }^{296}$. There are a number of considerations for the treatment of patients with haematological malignancies during the COVID-19 pandemic that apply to CAR T-cell therapy, such as considering deferral of treatment if ICU capacity may be overstretched and optimising prophylactic measures such as GCSF support ${ }^{317}$. Furthermore, recommendations specific to CAR T-cell therapy advocate the early use of tocilizumab in the management of CRS in order to mitigate the potential for serious toxicity requiring more intensive supportive care, and consider deferring B-NHL patients with disease features rendering them high risk to treatment failure, such as patients with an ECOG performance status $>2$ and markedly elevated serum LDH levels ${ }^{296}$. All of these recommendations should be considered when participants are being assessed for eligibility for or being treated on the ENABLE trial. However, having local CAR T-cell manufacturing capability within New Zealand, may significantly improve the logistical capability to deliver this cellular therapy during the COVID-19 global pandemic.

\subsection{Future direction for anti-CD19 CAR T-cell therapy}

There are multiple ways in which anti-CD19 CAR T-cell therapy could be improved upon in the future. In particular, developing methods to improve CAR T-cell manufacturing efficiency and feasibility, to reduce toxicities and to improve efficacy; are all key priorities for the future generation of anti-CD19 CAR T-cells.

The turnaround time from leukapheresis to CAR T-cell product infusion can be long for the currently licensed commercial anti-CD19 CAR T-cell therapies, with a median time of 54 days reported for tisagenlecleucel ${ }^{76}$. There are now a number of commercial and academic institutions evaluating ways to optimise the CAR T-cell manufacturing process, such as by adopting semi-automated, closed CAR T-cell manufacturing processes ${ }^{318-320}$. These methods may be able to improve the efficiency of the manufacturing process and lead to more uniform products by reducing manual manipulations in an open workflow. Adopting alternative methods for gene transduction is another strategy employed by some CAR T-cell manufacturing centres. The use of the transposon/transposase transduction system may have some advantages over the more widely adopted viral transduction systems, such as reduced cost and complexity of vector production, thereby potentially making this manufacturing 
method more scalable and exportable to serve large numbers of patients ${ }^{321} 322$. However, in comparison to lentiviral transduction systems as adopted in the ENABLE trial, the safety of the transposon/transposase system has not been as robustly evaluated to date, and the risk of insertional mutagenesis has been highlighted as a potential concern with this method of gene transduction $^{323} 324$.

The use of 'off-the-shelf' allogeneic anti-CD19 CAR T-cells could lead to significant improvements in manufacturing feasibility and ease of access to anti-CD19 CAR T-cell therapy ${ }^{250}$. Allogeneic CAR T-cells are usually created from healthy donors and are associated with the ability to make multiple doses of CAR T-cells from a single apheresis product, thereby potentially enabling multiple patients to access CAR T-cell treatment in a rapid timeframe ${ }^{250}$. There are significant challenges associated with the use of allogeneic CAR T-cells including graft versus host disease (GVHD) and donor rejection of allogeneic Tcells, both mediated by HLA mismatches between donor and recipient ${ }^{325} 326$. However, the use of virus specific HLA matched memory T-cells, non $\alpha \beta$ T-cells such as natural killer (NK) cells, or using gene editing methods to reduce TCR expression at the T-cell surface; show significant potential to reduce the risk of GVHD associated with allogeneic CAR Tcells $^{327-330}$. Results from a phase 1 and 2 trial where HLA-mismatched allogeneic anti-CD19 NK cells derived from cord blood were administers to 11 patients with $\mathrm{r} / \mathrm{r}$ B-cell malignancies including B-NHL, have recently been published ${ }^{272}$. In this study, Liu et al. reported that $73 \%$ of patients responded to treatment and there were no cases of CRS, neurotoxicity or GVHD associated with the treatment, demonstrating the potential for allogeneic anti-CD19 CAR NK cells to be a safe and feasible treatment for B-cell malignancies in the future ${ }^{272}$.

Acute anti-CD19 CAR T-cell related toxicities can be severe and represent a limitation of this modality of therapy ${ }^{71}$. The future generation of CARs may incorporate additional safety features to mitigate these toxicities ${ }^{331}$. For example, the inclusion of an inducible safety switch caspase 9 gene in a CAR which contains a modified human caspase 9 fused to the human FK506 binding protein, can lead to efficient elimination of CAR T-cells in vitro upon activation of this switch ${ }^{332}$. Using a humanised mouse model, the inducible caspase- 9 switch has been shown to eliminate anti-CD19 CAR T-cells in a dose dependent manner, allowing selective reduction of CAR T-cell expansion in the event of severe CRS ${ }^{333}$. There is now an anti-CD19 CAR T-cell trial for B-NHL underway which uses the inducible caspase 9 safety 
switch (clinicaltrials.gov number NCT03696784). Another method employed to eliminate CAR T-cells selectively in the event of severe toxicity, is to manufacture anti-CD19 CAR Tcells which co-express either CD20 or truncated epidermal growth factor receptor (EGFRt $)^{331}$. On administration of a clinically approved monoclonal antibody targeted against CD20 or EGFRt, the CAR T-cells can be eliminated through complement dependent cytotoxicity and/or antibody dependent cell-mediated toxicity ${ }^{334}$. Anti-CD19 CAR T-cells expressing EGFRt with the use of cetuximab as a suicide switch if severe toxicity develops, are currently being tested in a number of clinical trials in B-NHL (clinicaltrials.gov numbers NCT01865617, NCT03103971 and NCT02051257). Finally, CAR T-cells with cytokine neutralising capacity through expression of the membrane bound IL-6 receptor, have been shown to be highly effective in reducing IL-6 levels while maintaining anti-tumour efficacy in preclinical models, representing a potentially new approach to prevent CRS and supress its severity ${ }^{335}$.

There are numerous possible ways in which the efficacy of anti-CD19 CAR T-cell therapy in $\mathrm{r} / \mathrm{r}$ B-NHL may be optimised or improved in the future. The use of third generation antiCD19 CAR T-cells has been discussed at length in Chapter 2.6.4 Third generation CARs combining costimulatory domains, where there is evidence that third generation CAR T-cells can have increased expansion and proliferation in comparison to their second generation counterparts ${ }^{190}$. However, whether third generation anti-CD19 CAR T-cells can show additional anti-tumour efficacy without compromising safety requires further evaluation; results from other third generation anti-CD19 CAR T-cells such as the ENABLE trial, will hopefully be informative in answering this question in the future 261277336 . Furthermore, results reported by Ramos et al. showing substantial expansion of third generation anti-CD19 CAR T-cells even in the setting of minimal residual disease, are suggestive that third generation CAR T-cells may be particularly suited to the eradication of minimal residual disease ${ }^{190}$. Anti-CD19 CAR T-cells that also secrete cytokines such as IL-15 or IL-18 have been shown to increase in vivo CAR T-cell expansion and prolong survival in pre-clinical models; and maybe a useful strategy to improve clinical outcomes in $\mathrm{r} / \mathrm{r}$ B-NHL in the future $^{337}$. Combining anti-CD19 CAR T-cells with pharmacological agents such as immune checkpoint inhibitors or tyrosine kinase inhibitors, have shown potential in pre-clinical and clinical settings to improve CAR T-cell efficacy in the future ${ }^{80} 148$ 338-340. Clinical trials using anti-CD19 CAR T-cells that co-express a PD-1 blocking scFv have been shown to improve 
anti-tumour efficacy in pre-clinical models; and there are now clinical trials in patients with r/r B-NHL are underway (clinicaltrials.gov numbers NCT04163302 and NCT03932955) 339 .

\subsection{Conclusion}

Overall, the process of designing, setting up and preparing for this Phase 1 third generation anti-CD19 CAR T-cell trial for patients with r/r B-NHL; has provided a solid framework for this novel treatment modality to be used in New Zealand in the future. Establishing CAR Tcell manufacturing and treatment capability in New Zealand has taken a considerable multidisciplinary effort. The early experience of working on the ENABLE trial has demonstrated that the infrastructure required for CAR T-cell treatment is now in place in Wellington. The complexities of GMP CAR T-cell manufacturing are evident and the early experience gained from the ENABLE trial highlights the importance of evaluating feasibility of CAR T-cell manufacturing, as a key secondary endpoint of the study. If feasible CAR Tcell manufacturing can be established locally, this may have significant long term benefits to improve the logistical delivery of CAR T-cell therapy within New Zealand; this is particular relevant in the era of the COVID-19 global pandemic.

The treatment of many patients with r/r B-NHL remains an unmet clinical need within New Zealand. Establishing anti-CD19 CAR T-cell therapy in New Zealand provides a new treatment option with potential to effectively treat certain patients that are likely to be refractory or resistant to conventional therapies. The early experience of the ENABLE TMC, is that potential benefit for patients that may not even receive CAR T-cell therapy has been provided by reviewing these complex cases, and by advocating for compassionate access to other treatments. There has been successful collaboration with a number of international experts in terms of optimising CAR T-cell trial design, CAR T-cell manufacturing processes and developing CAR T-cell toxicity protocols based on the personal experience as well as the most up to date guidance. Through continued collaboration, CAR T-cell therapy will hopefully become a safe, feasible and effective treatment option for patients with $\mathrm{r} / \mathrm{r}$ B-NHL within New Zealand in the future. 
References

1. Brudno JN, Kochenderfer JN. Chimeric antigen receptor T-cell therapies for lymphoma. Nat Rev Clin Oncol 2018;15(1):31-46. doi: 10.1038/nrclinonc.2017.128

2. Eshhar Z, Waks T, Gross G, et al. Specific activation and targeting of cytotoxic lymphocytes through chimeric single chains consisting of antibody-binding domains and the gamma or zeta subunits of the immunoglobulin and T-cell receptors. Proc Nat/ Acad Sci U S A 1993;90(2):720-4. doi: 10.1073/pnas.90.2.720 [published Online First: 1993/01/15]

3. Gross G, Waks T, Eshhar Z. Expression of immunoglobulin-T-cell receptor chimeric molecules as functional receptors with antibody-type specificity. Proc Natl Acad Sci U S A 1989;86(24):10024-8. doi: 10.1073/pnas.86.24.10024 [published Online First: 1989/12/01]

4. Chen L, Flies DB. Molecular mechanisms of T cell co-stimulation and co-inhibition. Nat Rev Immunol 2013;13(4):227-42. doi: 10.1038/nri3405 [published Online First: 2013/03/09]

5. Turtle CJ, Hanafi LA, Berger C, et al. CD19 CAR-T cells of defined CD4+:CD8+ composition in adult B cell ALL patients. J Clin Invest 2016;126(6):2123-38. doi: 10.1172/JCI85309 [published Online First: 2016/04/26]

6. Kowolik CM, Topp MS, Gonzalez S, et al. CD28 costimulation provided through a CD19specific chimeric antigen receptor enhances in vivo persistence and antitumor efficacy of adoptively transferred T cells. Cancer research 2006;66(22):10995-1004. doi: 10.1158/0008-5472.Can-06-0160 [published Online First: 2006/11/17]

7. Kershaw MH, Westwood JA, Parker LL, et al. A phase I study on adoptive immunotherapy using gene-modified T cells for ovarian cancer. Clin Cancer Res 2006;12(20 Pt 1):610615. doi: 10.1158/1078-0432.Ccr-06-1183 [published Online First: 2006/10/26]

8. Till BG, Jensen MC, Wang J, et al. Adoptive immunotherapy for indolent non-Hodgkin lymphoma and mantle cell lymphoma using genetically modified autologous CD20specific T cells. Blood 2008;112(6):2261-71. doi: 10.1182/blood-2007-12-128843 [published Online First: 2008/05/30]

9. Savoldo B, Ramos CA, Liu E, et al. CD28 costimulation improves expansion and persistence of chimeric antigen receptor-modified T cells in lymphoma patients. $J$ Clin Invest 2011;121(5):1822-6. doi: 10.1172/jci46110 [published Online First: 2011/05/05]

10. Chow VA, Shadman M, Gopal AK. Translating anti-CD19 CAR T-cell therapy into clinical practice for relapsed/refractory diffuse large B-cell lymphoma. Blood 2018;132(8):777-81. doi: 10.1182/blood-2018-04-839217 [published Online First: 2018/06/20]

11. Neelapu SS, Locke FL, Bartlett NL, et al. Axicabtagene Ciloleucel CAR T-Cell Therapy in Refractory Large B-Cell Lymphoma. N Engl J Med 2017;377(26):2531-44. doi: 10.1056/NEJMoa1707447

12. Administration USFaD. FDA approves CAR-T cell therapy to treat adults with certain types of large B-cell lymphoma. FDA News Release: FDA, 2018. 
13. Novartis. Kymriah ${ }^{\circledR}$ (tisagenlecleucel), CAR-T therapy from Novartis, receives TGA approval for treating two aggressive blood cancers. Novartis Media Relations, December 2018.

14. McKee S. Gilead strikes deal with NHS England on Yescarta access. PharmaTimes Online 2018 8th October 2018.

15. Sommermeyer D, Hill T, Shamah SM, et al. Fully human CD19-specific chimeric antigen receptors for T-cell therapy. Leukemia 2017;31(10):2191-99. doi: 10.1038/leu.2017.57 [published Online First: 2017/02/17]

16. Guedan S, Posey AD, Jr., Shaw C, et al. Enhancing CAR T cell persistence through ICOS and 4-1BB costimulation. JCl insight 2018;3(1) doi: 10.1172/jci.insight.96976 [published Online First: 2018/01/13]

17. Lai $Y$, Weng J, Wei $X$, et al. Toll-like receptor 2 costimulation potentiates the antitumor efficacy of CAR T Cells. Leukemia 2018;32(3):801-08. doi: 10.1038/leu.2017.249 [published Online First: 2017/08/26]

18. Weng J, Lai P, Qin L, et al. A novel generation 1928zT2 CAR T cells induce remission in extramedullary relapse of acute lymphoblastic leukemia. J Hematol Oncol 2018;11(1):25. doi: 10.1186/s13045-018-0572-x [published Online First: 2018/02/21]

19. Fitzmaurice C, Allen C, Barber RM, et al. Global, Regional, and National Cancer Incidence, Mortality, Years of Life Lost, Years Lived With Disability, and Disability-Adjusted Lifeyears for 32 Cancer Groups, 1990 to 2015: A Systematic Analysis for the Global Burden of Disease Study. JAMA Oncol 2017;3(4):524-48. doi: 10.1001/jamaoncol.2016.5688 [published Online First: 2016/12/06]

20. Teras LR, DeSantis CE, Cerhan JR, et al. 2016 US lymphoid malignancy statistics by World Health Organization subtypes. CA Cancer J Clin 2016 doi: 10.3322/caac.21357 [published Online First: 2016/09/13]

21. Nowakowski GS, Blum KA, Kahl BS, et al. Beyond RCHOP: A Blueprint for Diffuse Large B Cell Lymphoma Research. J Natl Cancer Inst 2016;108(12) doi: 10.1093/jnci/djw257

22. Schneider C, Pasqualucci L, Dalla-Favera R. Molecular pathogenesis of diffuse large B-cell lymphoma. Semin Diagn Pathol 2011;28(2):167-77. doi:

10.1053/j.semdp.2011.04.001 [published Online First: 2011/08/17]

23. Savage KJ, Monti S, Kutok JL, et al. The molecular signature of mediastinal large B-cell lymphoma differs from that of other diffuse large B-cell lymphomas and shares features with classical Hodgkin lymphoma. Blood 2003;102(12):3871-9. doi: 10.1182/blood-2003-06-1841 [published Online First: 2003/08/23]

24. Swerdlow SH, Campo E, Pileri SA, et al. The 2016 revision of the World Health Organization classification of lymphoid neoplasms. Blood 2016;127(20):2375-90. doi: 10.1182/blood-2016-01-643569 [published Online First: 2016/03/17]

25. Saleh K, Michot JM, Camara-Clayette V, et al. Burkitt and Burkitt-Like Lymphomas: a Systematic Review. Curr Oncol Rep 2020;22(4):33. doi: 10.1007/s11912-020-0898-8 [published Online First: 2020/03/08]

26. Dada R. Diagnosis and management of follicular lymphoma: A comprehensive review. Eur J Haematol 2019;103(3):152-63. doi: 10.1111/ejh.13271 [published Online First: 2019/07/05]

27. Trotman J, Cheah CY, Marlton P, et al. Front-line management of non-Hodgkin lymphoma in Australia. Part 1: follicular lymphoma. Intern Med J 2019;49(4):422-33. doi: 10.1111/imj.14113 [published Online First: 2018/09/20] 
28. Maddocks K. Update on mantle cell lymphoma. Blood 2018;132(16):1647-56. doi: 10.1182/blood-2018-03-791392 [published Online First: 2018/08/30]

29. Molina TJ, Canioni D, Copie-Bergman C, et al. Young patients with non-germinal center Bcell-like diffuse large B-cell lymphoma benefit from intensified chemotherapy with ACVBP plus rituximab compared with CHOP plus rituximab: analysis of data from the Groupe 039;Etudes des Lymphomes de 039;Adulte/lymphoma study association phase III trial LNH 03-2B. Journal of Clinical Oncology 2014;32(35):3996. doi: 10.1200/JCO.2013.54.9493

30. Coiffier B, Sarkozy C. Diffuse large B-cell lymphoma: R-CHOP failure-what to do? Hematology Am Soc Hematol Educ Program 2016;2016(1):366-78. doi: 10.1182/asheducation-2016.1.366

31. Friedberg JW. How I treat double-hit lymphoma. Blood 2017;130(5):590-96. doi: 10.1182/blood-2017-04-737320 [published Online First: 2017/06/11]

32. Dunleavy K, Fanale MA, Abramson JS, et al. Dose-adjusted EPOCH-R (etoposide, prednisone, vincristine, cyclophosphamide, doxorubicin, and rituximab) in untreated aggressive diffuse large B-cell lymphoma with MYC rearrangement: a prospective, multicentre, single-arm phase 2 study. Lancet Haematol 2018;5(12):e609-e17. doi: 10.1016/s2352-3026(18)30177-7 [published Online First: 2018/12/07]

33. Flinn IW, van der Jagt $R$, Kahl BS, et al. Randomized trial of bendamustine-rituximab or RCHOP/R-CVP in first-line treatment of indolent NHL or MCL: the BRIGHT study. Blood 2014;123(19):2944-52. doi: 10.1182/blood-2013-11-531327 [published Online First: 2014/03/05]

34. Geisler CH, Kolstad A, Laurell A, et al. Nordic MCL2 trial update: six-year follow-up after intensive immunochemotherapy for untreated mantle cell lymphoma followed by BEAM or BEAC + autologous stem-cell support: still very long survival but late relapses do occur. Br J Haematol 2012;158(3):355-62. doi: 10.1111/j.1365-2141.2012.09174.x [published Online First: 2012/05/30]

35. Gisselbrecht C, Glass B, Mounier N, et al. Salvage regimens with autologous transplantation for relapsed large B-cell lymphoma in the rituximab era. J Clin Oncol 2010;28(27):4184-90. doi: 10.1200/jco.2010.28.1618 [published Online First: 2010/07/28]

36. Crump M, Neelapu SS, Farooq U, et al. Outcomes in refractory diffuse large B-cell lymphoma: results from the international SCHOLAR-1 study. Blood 2017;130(16):1800-08. doi: 10.1182/blood-2017-03-769620 [published Online First: 2017/08/05]

37. Herrera AF, Mei M, Low L, et al. Relapsed or Refractory Double-Expressor and Double-Hit Lymphomas Have Inferior Progression-Free Survival After Autologous Stem-Cell Transplantation. J Clin Oncol 2017;35(1):24-31. doi: 10.1200/jco.2016.68.2740 [published Online First: 2016/12/31]

38. Godfrey J, Leukam MJ, Smith SM. An update in treating transformed lymphoma. Best practice \& research Clinical haematology 2018;31(3):251-61. doi: 10.1016/j.beha.2018.07.008 [published Online First: 2018/09/15]

39. Wirk B, Fenske TS, Hamadani M, et al. Outcomes of hematopoietic cell transplantation for diffuse large B cell lymphoma transformed from follicular lymphoma. Biology of blood and marrow transplantation : journal of the American Society for Blood and Marrow Transplantation 2014;20(7):951-9. doi: 10.1016/j.bbmt.2014.03.014 [published Online First: 2014/03/20] 
40. Escobar IG, Sanchez de Ibarguen BC, de Juan VC, et al. High-dose chemotherapy followed by autologous and allogeneic hematopoietic stem cell transplantation in patients with follicular non-Hodgkin's lymphoma in the rituximab era. Tumori 2015;101(1):2-7. doi: 10.5301/tj.5000203 [published Online First: 2015/02/24]

41. Erblich T, Montoto S. Treating relapsed follicular lymphoma. Expert Rev Hematol 2018;11(5):403-10. doi: 10.1080/17474086.2018.1453801 [published Online First: 2018/03/16]

42. Jurinovic $V$, Kridel R, Staiger AM, et al. Clinicogenetic risk models predict early progression of follicular lymphoma after first-line immunochemotherapy. Blood 2016;128(8):1112-20. doi: 10.1182/blood-2016-05-717355 [published Online First: 2016/07/16]

43. Kuruvilla J. The role of autologous and allogeneic stem cell transplantation in the management of indolent B-cell lymphoma. Blood 2016;127(17):2093-100. doi: 10.1182/blood-2015-11-624320 [published Online First: 2016/03/19]

44. Klyuchnikov E, Bacher U, Kroger NM, et al. Reduced-Intensity Allografting as First Transplantation Approach in Relapsed/Refractory Grades One and Two Follicular Lymphoma Provides Improved Outcomes in Long-Term Survivors. Biology of blood and marrow transplantation : journal of the American Society for Blood and Marrow Transplantation 2015;21(12):2091-99. doi: 10.1016/j.bbmt.2015.07.028 [published Online First: 2015/08/09]

45. Evens AM, Vanderplas A, LaCasce AS, et al. Stem cell transplantation for follicular lymphoma relapsed/refractory after prior rituximab: a comprehensive analysis from the NCCN lymphoma outcomes project. Cancer 2013;119(20):3662-71. doi: 10.1002/cncr.28243 [published Online First: 2013/08/08]

46. Norman JE, Schouten HC, Dreger $P$, et al. The role of stem cell transplantation in the management of relapsed follicular lymphoma in the era of targeted therapies. Bone marrow transplantation 2018 doi: 10.1038/s41409-018-0372-5 [published Online First: 2018/11/06]

47. Sureda A, Dreger P, Bishop MR, et al. Prevention and treatment of relapse after stem cell transplantation in lymphoid malignancies. Bone marrow transplantation 2019;54(1):17-25. doi: 10.1038/s41409-018-0214-5 [published Online First: 2018/05/26]

48. Campo E, Rule S. Mantle cell lymphoma: evolving management strategies. Blood 2015;125(1):48-55. doi: 10.1182/blood-2014-05-521898 [published Online First: 2014/12/17]

49. Cohen JB, Burns LJ, Bachanova V. Role of allogeneic stem cell transplantation in mantle cell lymphoma. European journal of haematology 2015;94(4):290-7. doi: 10.1111/ejh.12442 [published Online First: 2014/08/27]

50. Cook G, Smith GM, Kirkland K, et al. Outcome following Reduced-Intensity Allogeneic Stem Cell Transplantation (RIC AlloSCT) for relapsed and refractory mantle cell lymphoma (MCL): a study of the British Society for Blood and Marrow Transplantation. Biology of blood and marrow transplantation : journal of the American Society for Blood and Marrow Transplantation 2010;16(10):1419-27. doi: 10.1016/j.bbmt.2010.04.006 [published Online First: 2010/04/20]

51. Dobosz P, Dzieciatkowski T. The Intriguing History of Cancer Immunotherapy. Front Immunol 2019;10:2965. doi: 10.3389/fimmu.2019.02965 [published Online First: 2020/01/11] 
52. Rosenberg SA, Spiess $P$, Lafreniere R. A new approach to the adoptive immunotherapy of cancer with tumor-infiltrating lymphocytes. Science 1986;233(4770):1318-21. doi: 10.1126/science.3489291 [published Online First: 1986/09/19]

53. Rosenberg SA, Packard BS, Aebersold PM, et al. Use of tumor-infiltrating lymphocytes and interleukin-2 in the immunotherapy of patients with metastatic melanoma. A preliminary report. N Engl J Med 1988;319(25):1676-80. doi: 10.1056/nejm198812223192527 [published Online First: 1988/12/22]

54. Zhang H, Lim HS, Knapp B, et al. The contribution of major histocompatibility complex contacts to the affinity and kinetics of T cell receptor binding. Sci Rep 2016;6:35326. doi: 10.1038/srep35326 [published Online First: 2016/10/14]

55. Kebriaei $P$, Singh $H$, Huls MH, et al. Phase I trials using Sleeping Beauty to generate CD19specific CAR T cells. J Clin Invest 2016;126(9):3363-76. doi: 10.1172/jci86721 [published Online First: 2016/08/03]

56. Manuri PV, Wilson MH, Maiti SN, et al. piggyBac transposon/transposase system to generate CD19-specific T cells for the treatment of B-lineage malignancies. Hum Gene Ther 2010;21(4):427-37. doi: 10.1089/hum.2009.114 [published Online First: 2009/11/13]

57. Cornetta K, Yao J, Jasti A, et al. Replication-competent lentivirus analysis of clinical grade vector products. Mol Ther 2011;19(3):557-66. doi: 10.1038/mt.2010.278 [published Online First: 2010/12/24]

58. White M, Whittaker R, Gandara C, et al. A Guide to Approaching Regulatory Considerations for Lentiviral-Mediated Gene Therapies. Hum Gene Ther Methods 2017;28(4):163-76. doi: 10.1089/hgtb.2017.096 [published Online First: 2017/08/18]

59. Milone MC, O'Doherty U. Clinical use of lentiviral vectors. Leukemia 2018;32(7):1529-41. doi: 10.1038/s41375-018-0106-0 [published Online First: 2018/04/15]

60. Morgan RA, Boyerinas B. Genetic Modification of T Cells. Biomedicines 2016;4(2) doi: 10.3390/biomedicines4020009 [published Online First: 2017/05/26]

61. Scholler J, Brady TL, Binder-Scholl G, et al. Decade-long safety and function of retroviralmodified chimeric antigen receptor $T$ cells. Science translational medicine 2012;4(132):132ra53. doi: 10.1126/scitransImed.3003761 [published Online First: 2012/05/04]

62. Long AH, Haso WM, Shern JF, et al. 4-1BB costimulation ameliorates T cell exhaustion induced by tonic signaling of chimeric antigen receptors. Nature medicine 2015;21(6):581-90. doi: 10.1038/nm.3838 [published Online First: 2015/05/06]

63. Yescarta (R) [package insert]. Kite Pharma, Inc. Santa Monica, CA, USA, 2017.

64. Kymriah (R) [package insert]. East Hanover,

New Jersey 07936.: Novartis Pharmaceuticals Corporation,, 2018.

65. Turtle CJ, Riddell SR, Maloney DG. CD19-Targeted chimeric antigen receptor-modified Tcell immunotherapy for B-cell malignancies. Clinical pharmacology and therapeutics 2016;100(3):252-8. doi: 10.1002/cpt.392 [published Online First: 2016/05/14]

66. Brentjens RJ, Riviere I, Park JH, et al. Safety and persistence of adoptively transferred autologous CD19-targeted T cells in patients with relapsed or chemotherapy refractory B-cell leukemias. Blood 2011;118(18):4817-28. doi: 10.1182/blood-201104-348540 [published Online First: 2011/08/19]

67. Blanc V, Bousseau A, Caron A, et al. SAR3419: an anti-CD19-Maytansinoid Immunoconjugate for the treatment of B-cell malignancies. Clin Cancer Res 
2011;17(20):6448-58. doi: 10.1158/1078-0432.Ccr-11-0485 [published Online First:

2011/10/18]

68. June $\mathrm{CH}, \mathrm{O}$ 'Connor RS, Kawalekar OU, et al. CAR T cell immunotherapy for human cancer. Science 2018;359(6382):1361-65. doi: 10.1126/science.aar6711 [published Online First: 2018/03/24]

69. Daniyan AF, Brentjens RJ. At the Bench: Chimeric antigen receptor (CAR) T cell therapy for the treatment of B cell malignancies. J Leukoc Biol 2016;100(6):1255-64. doi:

10.1189/jlb.5BT1215-556RR [published Online First: 2016/10/30]

70. van Zelm MC, Reisli I, van der Burg M, et al. An antibody-deficiency syndrome due to mutations in the CD19 gene. N Eng/ J Med 2006;354(18):1901-12. doi:

10.1056/NEJMoa051568 [published Online First: 2006/05/05]

71. Brudno JN, Kochenderfer JN. Recent advances in CAR T-cell toxicity: Mechanisms, manifestations and management. Blood Rev 2019;34:45-55. doi: 10.1016/j.blre.2018.11.002 [published Online First: 2018/12/12]

72. Cheng J, Zhao L, Zhang Y, et al. Understanding the Mechanisms of Resistance to CAR TCell Therapy in Malignancies. Front Oncol 2019;9:1237. doi: 10.3389/fonc.2019.01237 [published Online First: 2019/12/12]

73. Advani AS, Hanna R. The treatment of adolescents and young adults with acute lymphoblastic leukemia. Leuk Lymphoma 2020;61(1):18-26. doi: 10.1080/10428194.2019.1658103 [published Online First: 2019/08/28]

74. June CH, Sadelain M. Chimeric Antigen Receptor Therapy. N Eng/ J Med 2018;379(1):6473. doi: 10.1056/NEJMra1706169 [published Online First: 2018/07/05]

75. Maus MV, Grupp SA, Porter DL, et al. Antibody-modified T cells: CARs take the front seat for hematologic malignancies. Blood 2014;123(17):2625-35. doi: 10.1182/blood2013-11-492231 [published Online First: 2014/03/01]

76. Schuster SJ, Bishop MR, Tam CS, et al. Tisagenlecleucel in Adult Relapsed or Refractory Diffuse Large B-Cell Lymphoma. N Engl J Med 2018 doi: 10.1056/NEJMoa1804980 [published Online First: 2018/12/07]

77. Abramson JSP, M. L.; Gordon, L. I.; Lunning. M. A.; Wang. M. L.; Arnason. J. E et al. Pivotal Safety and Efficacy Results from Transcend NHL 001, a Multicenter Phase 1 Study of Lisocabtagene Maraleucel (liso-cel) in Relapsed/Refractory (R/R) Large B Cell Lymphomas. ASH 2019. Orlando, Florida: Blood, 2019:241.

78. Locke FL, Ghobadi A, Jacobson CA, et al. Long-term safety and activity of axicabtagene ciloleucel in refractory large B-cell lymphoma (ZUMA-1): a single-arm, multicentre, phase 1-2 trial. The Lancet Oncology 2019;20(1):31-42. doi: 10.1016/s14702045(18)30864-7 [published Online First: 2018/12/07]

79. Nastoupil LJJ, M, D.; Spiegel, J. Y et al. Axicabtagene Ciloleucel (Axi-cel) CD19 Chimeric Antigen Receptor (CAR) T-Cell Therapy for Relapsed/Refractory Large B-Cell Lymphoma: Real World Experience. American Society of Haematology. San Diego: Blood, 2018:91.

80. Abramson JS. Anti-CD19 CAR T-Cell Therapy for B-Cell Non-Hodgkin Lymphoma. Transfus Med Rev 2020;34(1):29-33. doi: 10.1016/j.tmrv.2019.08.003 [published Online First: 2019/11/05]

81. Kochenderfer JN, Wilson WH, Janik JE, et al. Eradication of B-lineage cells and regression of lymphoma in a patient treated with autologous T cells genetically engineered to recognize CD19. Blood 2010;116(20):4099-102. doi: 10.1182/blood-2010-04-281931 [published Online First: 2010/07/30] 
82. Kochenderfer JN, Somerville RPT, Lu T, et al. Lymphoma Remissions Caused by Anti-CD19 Chimeric Antigen Receptor T Cells Are Associated With High Serum Interleukin-15 Levels. J Clin Oncol 2017;35(16):1803-13. doi: 10.1200/jco.2016.71.3024 [published Online First: 2017/03/16]

83. Schuster SJ, Svoboda J, Chong EA, et al. Chimeric Antigen Receptor T Cells in Refractory BCell Lymphomas. New England Journal of Medicine 2017;377(26):2545-54. doi: 10.1056/NEJMoa1708566

84. Hirayama AV, Gauthier J, Hay KA, et al. High rate of durable complete remission in follicular lymphoma after CD19 CAR-T cell immunotherapy. Blood 2019;134(7):63640. doi: 10.1182/blood.2019000905 [published Online First: 2019/10/28]

85. Bishop MR. The case for CAR T-cell therapy in follicular lymphomas. Blood 2019;134(7):577-78. doi: 10.1182/blood.2019001843 [published Online First: 2019/08/17]

86. Turtle CJ, Hanafi LA, Berger C, et al. Immunotherapy of non-Hodgkin's lymphoma with a defined ratio of CD8+ and CD4+ CD19-specific chimeric antigen receptor-modified $T$ cells. Science translational medicine 2016;8(355):355ra116. doi: 10.1126/scitranslmed.aaf8621 [published Online First: 2016/09/09]

87. Wang M, Munoz J, Goy A, et al. KTE-X19 CAR T-Cell Therapy in Relapsed or Refractory Mantle-Cell Lymphoma. N Eng/ J Med 2020;382(14):1331-42. doi: 10.1056/NEJMoa1914347 [published Online First: 2020/04/04]

88. Riedell PAW, C.; Nastoupil, L. J et al. A Multicenter Retrospective Analysis of Clinical Outcomes, Toxicities, and Patterns of Use in Institutions Utilizing Commercial Axicabtagene Ciloleucel and Tisagenlecleucel for Relapsed/Refractory Aggressive BCell Lymphomas. American Society of Haematology Annual Meeting. Orlando: Blood, 2019:1599.

89. Kuhnl A, Roddie C, Martinez-Cibrian N, et al. Real-World Data of High-Grade Lymphoma Patients Treated with CD19 CAR-T in England. Blood 2019;134(Supplement_1):76767. doi: 10.1182/blood-2019-124177

90. Kuhnl AR, C.; Martinez-Cibrin, N.; Menne, T. F.; Linton, K et al. Real-World Data of HighGrade Lymphoma Patients Treated with CD19 CAR-T in England. American Society of Haematology Annual Conference. Orlando: Blood, 2019:767.

91. Zhang X, Li JJ, Lu PH. Advances in the development of chimeric antigen receptor-T-cell therapy in B-cell acute lymphoblastic leukemia. Chin Med J (Engl) 2020 doi: 10.1097/cm9.0000000000000638 [published Online First: 2020/01/25]

92. Kochenderfer JN, Somerville RPT, Lu T, et al. Long-Duration Complete Remissions of Diffuse Large B Cell Lymphoma after Anti-CD19 Chimeric Antigen Receptor T Cell Therapy. Mol Ther 2017;25(10):2245-53. doi: 10.1016/j.ymthe.2017.07.004 [published Online First: 2017/08/15]

93. Kunkele A, Johnson AJ, Rolczynski LS, et al. Functional Tuning of CARs Reveals Signaling Threshold above Which CD8+ CTL Antitumor Potency Is Attenuated due to Cell FasFasL-Dependent AICD. Cancer Immunol Res 2015;3(4):368-79. doi: 10.1158/23266066.Cir-14-0200 [published Online First: 2015/01/13]

94. Alabanza L, Pegues M, Geldres C, et al. Function of Novel Anti-CD19 Chimeric Antigen Receptors with Human Variable Regions Is Affected by Hinge and Transmembrane Domains. Mol Ther 2017;25(11):2452-65. doi: 10.1016/j.ymthe.2017.07.013 [published Online First: 2017/08/16] 
95. Riaz N, Havel JJ, Makarov V, et al. Tumor and Microenvironment Evolution during Immunotherapy with Nivolumab. Cell 2017;171(4):934-49.e16. doi: 10.1016/j.cell.2017.09.028 [published Online First: 2017/10/17]

96. Xu-Monette ZY, Zhou J, Young KH. PD-1 expression and clinical PD-1 blockade in B-cell lymphomas. Blood 2018;131(1):68-83. doi: 10.1182/blood-2017-07-740993 [published Online First: 2017/11/10]

97. Zolov SN, Rietberg SP, Bonifant CL. Programmed cell death protein 1 activation preferentially inhibits CD28.CAR-T cells. Cytotherapy 2018;20(10):1259-66. doi: 10.1016/j.jcyt.2018.07.005 [published Online First: 2018/10/13]

98. John LB, Kershaw MH, Darcy PK. Blockade of PD-1 immunosuppression boosts CAR T-cell therapy. Oncoimmunology 2013;2(10):e26286. doi: 10.4161/onci.26286 [published Online First: 2013/12/20]

99. Yoon DH, Osborn MJ, Tolar J, et al. Incorporation of Immune Checkpoint Blockade into Chimeric Antigen Receptor T Cells (CAR-Ts): Combination or Built-In CAR-T. International journal of molecular sciences 2018;19(2) doi: 10.3390/ijms19020340 [published Online First: 2018/01/25]

100. The effect of pembrolizumab in combination with CD19-targeted chimeric antigen receptor (CAR) T cells in relapsed acute lymphoblastic leukemia (ALL). American Society of Clinical Oncology; 2017. Journal of Clincal Oncology.

101. Chong EA, Melenhorst JJ, Lacey SF, et al. PD-1 blockade modulates chimeric antigen receptor (CAR)-modified T cells: refueling the CAR. Blood 2017;129(8):1039-41. doi: 10.1182/blood-2016-09-738245 [published Online First: 2016/12/30]

102. Dickinson M, Weinkove R. Maintaining a fit T-cell compartment: lymphoma treatment sequencing in the era of chimeric antigen receptor T-cell therapies. Intern Med J 2019;49(10):1338. doi: 10.1111/imj.14453 [published Online First: 2019/10/12]

103. Fraietta JA, Lacey SF, Orlando EJ, et al. Determinants of response and resistance to CD19 chimeric antigen receptor (CAR) T cell therapy of chronic lymphocytic leukemia. Nat Med 2018;24(5):563-71. doi: 10.1038/s41591-018-0010-1 [published Online First: 2018/05/02]

104. Jensen MC, Popplewell L, Cooper L, et al. Antitransgene rejection responses contribute to attenuated persistence of adoptively transferred CD20/CD19-specific chimeric antigen receptor redirected T cells in humans. Biology of blood and marrow transplantation : journal of the American Society for Blood and Marrow Transplantation 2010;16(9):1245-56. doi: 10.1016/j.bbmt.2010.03.014 [published Online First: 2010/03/23]

105. Lee DW, Kochenderfer JN, Stetler-Stevenson M, et al. T cells expressing CD19 chimeric antigen receptors for acute lymphoblastic leukaemia in children and young adults: a phase 1 dose-escalation trial. Lancet 2015;385(9967):517-28. doi: 10.1016/S01406736(14)61403-3 [published Online First: 2014/10/17]

106. Cahill KE, Leukam MJ, Riedell PA. Refining patient selection for CAR T-cell therapy in aggressive large B-cell lymphoma. Leuk Lymphoma 2020;61(4):799-807. doi: 10.1080/10428194.2019.1691201 [published Online First: 2019/11/22]

107. Jacobson CAH, B.; Armand, P et al. Axicabtagene ciloleucel in the real world: outcomes and predictors of response, resistance and toxicity. American Society of Haematology. San Diego: Blood, 2018.

108. Locke Fea. Outcomes by prior lines of therapy (LoT) in ZUMA-1, the pivotal phase 2 study of axicabtagene ciloleucel (Axi-Cel) in patients (Pts) with refractory large B cell 
lymphoma. American Society of Clinical Oncology: Journal of Clinical Oncology, 2018:3039.

109. Neelapu SS, Tummala S, Kebriaei P, et al. Chimeric antigen receptor T-cell therapy assessment and management of toxicities. Nature reviews Clinical oncology 2018;15(1):47-62. doi: 10.1038/nrclinonc.2017.148 [published Online First: 2017/09/20]

110. Assi R, Kantarjian H, Short NJ, et al. Safety and Efficacy of Blinatumomab in Combination With a Tyrosine Kinase Inhibitor for the Treatment of Relapsed Philadelphia Chromosome-positive Leukemia. Clin Lymphoma Myeloma Leuk 2017;17(12):897901. doi: 10.1016/j.clml.2017.08.101 [published Online First: 2017/09/21]

111. Wang Z, Han W. Biomarkers of cytokine release syndrome and neurotoxicity related to CAR-T cell therapy. Biomark Res 2018;6:4. doi: 10.1186/s40364-018-0116-0 [published Online First: 2018/02/02]

112. Walker M, Makropoulos D, Achuthanandam R, et al. Recent advances in the understanding of drug-mediated infusion reactions and cytokine release syndrome. Curr Opin Drug Discov Devel 2010;13(1):124-35. [published Online First: 2010/01/05]

113. Hay KA, Hanafi LA, Li D, et al. Kinetics and biomarkers of severe cytokine release syndrome after CD19 chimeric antigen receptor-modified T-cell therapy. Blood 2017;130(21):2295-306. doi: 10.1182/blood-2017-06-793141 [published Online First: 2017/09/20]

114. Hay KA. Cytokine release syndrome and neurotoxicity after CD19 chimeric antigen receptor-modified (CAR-) T cell therapy. Br J Haematol 2018;183(3):364-74. doi: 10.1111/bjh.15644 [published Online First: 2018/11/09]

115. Wu C, Xue Y, Wang P, et al. IFN-gamma primes macrophage activation by increasing phosphatase and tensin homolog via downregulation of miR-3473b. J Immunol 2014;193(6):3036-44. doi: 10.4049/jimmunol.1302379 [published Online First: 2014/08/06]

116. Singh N, Hofmann TJ, Gershenson Z, et al. Monocyte lineage-derived IL- 6 does not affect chimeric antigen receptor T-cell function. Cytotherapy 2017;19(7):867-80. doi: 10.1016/j.jcyt.2017.04.001 [published Online First: 2017/05/17]

117. Norelli M, Camisa B, Barbiera G, et al. Monocyte-derived IL-1 and IL-6 are differentially required for cytokine-release syndrome and neurotoxicity due to CAR T cells. Nat Med 2018;24(6):739-48. doi: 10.1038/s41591-018-0036-4 [published Online First: 2018/05/29]

118. Teachey DT, Rheingold SR, Maude SL, et al. Cytokine release syndrome after blinatumomab treatment related to abnormal macrophage activation and ameliorated with cytokine-directed therapy. Blood. 2013;121(26):5154-5157. Blood 2016;128(10):1441. doi: 10.1182/blood-2016-07-730671 [published Online First: 2016/09/08]

119. Obstfeld AE, Frey NV, Mansfield K, et al. Cytokine release syndrome associated with chimeric-antigen receptor T-cell therapy: clinicopathological insights. Blood 2017;130(23):2569-72. doi: 10.1182/blood-2017-08-802413 [published Online First: 2017/10/28]

120. Lee DW, Gardner R, Porter DL, et al. Current concepts in the diagnosis and management of cytokine release syndrome. Blood 2014;124(2):188-95. doi: 10.1182/blood-201405-552729 
121. Alvi RM, Frigault MJ, Fradley MG, et al. Cardiovascular Events Among Adults Treated With Chimeric Antigen Receptor T-Cells (CAR-T). J Am Coll Cardiol 2019;74(25):3099108. doi: 10.1016/j.jacc.2019.10.038 [published Online First: 2019/12/21]

122. Maude SL, Barrett D, Teachey DT, et al. Managing cytokine release syndrome associated with novel T cell-engaging therapies. Cancer journal (Sudbury, Mass) 2014;20(2):11922. doi: 10.1097/ppo.0000000000000035 [published Online First: 2014/03/29]

123. Maude SL, Frey N, Shaw PA, et al. Chimeric antigen receptor T cells for sustained remissions in leukemia. N Engl J Med 2014;371(16):1507-17. doi: 10.1056/NEJMoa1407222 [published Online First: 2014/10/16]

124. Porter D, Frey N, Wood PA, et al. Grading of cytokine release syndrome associated with the CAR T cell therapy tisagenlecleucel. J Hematol Oncol 2018;11(1):35. doi: 10.1186/s13045-018-0571-y [published Online First: 2018/03/04]

125. Porter DL, Hwang WT, Frey NV, et al. Chimeric antigen receptor T cells persist and induce sustained remissions in relapsed refractory chronic lymphocytic leukemia. Sci Trans/ Med 2015;7(303):303ra139. doi: 10.1126/scitransImed.aac5415 [published Online First: 2015/09/04]

126. Lee DW, Santomasso BD, Locke FL, et al. ASTCT Consensus Grading for Cytokine Release Syndrome and Neurologic Toxicity Associated with Immune Effector Cells. Biol Blood Marrow Transplant 2019;25(4):625-38. doi: 10.1016/j.bbmt.2018.12.758 [published Online First: 2018/12/29]

127. Teachey DT, Lacey SF, Shaw PA, et al. Identification of Predictive Biomarkers for Cytokine Release Syndrome after Chimeric Antigen Receptor T-cell Therapy for Acute Lymphoblastic Leukemia. Cancer discovery 2016;6(6):664-79. doi: 10.1158/21598290.Cd-16-0040 [published Online First: 2016/04/15]

128. Park JH, Riviere I, Gonen M, et al. Long-Term Follow-up of CD19 CAR Therapy in Acute Lymphoblastic Leukemia. N Eng/J Med 2018;378(5):449-59. doi:

10.1056/NEJMoa1709919 [published Online First: 2018/02/01]

129. Borchmann P. An updated analysis of JULIET, a global pivotal phase 2 trial of tisagenlecleucel in adult patients with relapsed or refractory $(R / R)$ diffuse large B-cell lymphoma (DLBCL). 23rd Congress of European Haematology Association. Stockholm, Sweeden, 2018.

130. Gust J, Hay KA, Hanafi LA, et al. Endothelial Activation and Blood-Brain Barrier Disruption in Neurotoxicity after Adoptive Immunotherapy with CD19 CAR-T Cells. Cancer Discov 2017;7(12):1404-19. doi: 10.1158/2159-8290.Cd-17-0698 [published Online First: 2017/10/14]

131. Neelapu SS, Tummala S, Kebriaei $P$, et al. Toxicity management after chimeric antigen receptor T cell therapy: one size does not fit 'ALL'. Nat Rev Clin Oncol 2018;15(4):218. doi: 10.1038/nrclinonc.2018.20 [published Online First: 2018/02/13]

132. Awasthi R. Clinical pharmacology of tisagenlecleucel (CTLO19) in patients with relapsed/refractory ( $r / r$ ) diffuse large B Cell lymphoma (DLBCL). AACR Annual Meetin, 2018.

133. Milone MC, Bhoj VG. The Pharmacology of T Cell Therapies. Mol Ther Methods Clin Dev 2018;8:210-21. doi: 10.1016/j.omtm.2018.01.010 [published Online First: 2018/03/20]

134. Dasyam N, George P, Weinkove R. Chimeric antigen receptor T-cell therapies: Optimising the dose. Br J Clin Pharmacol 2020 doi: 10.1111/bcp.14281 [published Online First: 2020/03/17] 
135. Locke FL, Neelapu SS, Bartlett NL, et al. Phase 1 Results of ZUMA-1: A Multicenter Study of KTE-C19 Anti-CD19 CAR T Cell Therapy in Refractory Aggressive Lymphoma.

Molecular therapy: the journal of the American Society of Gene Therapy 2017;25(1):285-95. doi: 10.1016/j.ymthe.2016.10.020 [published Online First: 2017/01/28]

136. Maude SL, Laetsch TW, Buechner J, et al. Tisagenlecleucel in Children and Young Adults with B-Cell Lymphoblastic Leukemia. N Engl J Med 2018;378(5):439-48. doi: 10.1056/NEJMoa1709866 [published Online First: 2018/02/01]

137. Perica K, Curran KJ, Brentjens RJ, et al. Building a CAR Garage: Preparing for the Delivery of Commercial CAR T Cell Products at Memorial Sloan Kettering Cancer Center. Biol Blood Marrow Transplant 2018 doi: 10.1016/j.bbmt.2018.02.018 [published Online First: 2018/03/03]

138. Le RQ, Li L, Yuan W, et al. FDA Approval Summary: Tocilizumab for Treatment of Chimeric Antigen Receptor T Cell-Induced Severe or Life-Threatening Cytokine Release Syndrome. Oncologist 2018 doi: 10.1634/theoncologist.2018-0028 [published Online First: 2018/04/07]

139. Administration USFaD. Approved risk evaluation and mitigation strategies (REMS). Kymriah (tisagenlecleucel). 2018.

140. Administration USFaD. Approved risk evaluation and mitigation strategies (REMS). Yescarta (axicabtagene ciloleucel), 2018.

141. Yanez L, Sanchez-Escamilla M, Perales MA. CAR T Cell Toxicity: Current Management and Future Directions. Hemasphere 2019;3(2):e186. doi: 10.1097/hs9.0000000000000186 [published Online First: 2019/11/15]

142. Lanza L, Scudeletti M, Puppo F, et al. Prednisone increases apoptosis in in vitro activated human peripheral blood T lymphocytes. Clin Exp Immunol 1996;103(3):482-90. doi: 10.1111/j.1365-2249.1996.tb08306.x [published Online First: 1996/03/01]

143. Paliogianni F, Ahuja SS, Balow JP, et al. Novel mechanism for inhibition of human T cells by glucocorticoids. Glucocorticoids inhibit signal transduction through IL-2 receptor. J Immunol 1993;151(8):4081-9. [published Online First: 1993/10/15]

144. Gardner RL, K, J. Decreased Rates of Severe CRS Seen with Early Intervention Strategies for CD19 CAR-T Cell Toxicity Management. ASH: Blood, 2016:586.

145. Gardner RA, Ceppi F, Rivers J, et al. Preemptive mitigation of CD19 CAR T-cell cytokine release syndrome without attenuation of antileukemic efficacy. Blood 2019;134(24):2149-58. doi: 10.1182/blood.2019001463 [published Online First: 2019/11/08]

146. Liu S, Deng B, Yin Z, et al. Corticosteroids do not influence the efficacy and kinetics of CAR-T cells for B-cell acute lymphoblastic leukemia. Blood Cancer J 2020;10(2):15. doi: 10.1038/s41408-020-0280-y [published Online First: 2020/02/08]

147. Mestermann K, Giavridis T, Weber J, et al. The tyrosine kinase inhibitor dasatinib acts as a pharmacologic on/off switch for CAR T cells. Sci Trans/ Med 2019;11(499) doi: 10.1126/scitranslmed.aau5907 [published Online First: 2019/07/05]

148. Turtle CJ, Hay KA, Hanafi LA, et al. Durable Molecular Remissions in Chronic Lymphocytic Leukemia Treated With CD19-Specific Chimeric Antigen Receptor-Modified T Cells After Failure of Ibrutinib. J Clin Oncol 2017;35(26):3010-20. doi: 10.1200/jco.2017.72.8519 [published Online First: 2017/07/18]

149. Lee DW, Santomasso BD, Locke FL, et al. ASBMT Consensus Grading for Cytokine Release Syndrome and Neurologic Toxicity Associated with Immune Effector Cells. Biology of 
blood and marrow transplantation : journal of the American Society for Blood and Marrow Transplantation 2018 doi: 10.1016/j.bbmt.2018.12.758 [published Online First: 2018/12/29]

150. Santomasso BD, Park JH, Salloum D, et al. Clinical and Biological Correlates of Neurotoxicity Associated with CAR T-cell Therapy in Patients with B-cell Acute Lymphoblastic Leukemia. Cancer Discov 2018;8(8):958-71. doi: 10.1158/21598290.Cd-17-1319 [published Online First: 2018/06/09]

151. Reiber H. Flow rate of cerebrospinal fluid (CSF)--a concept common to normal blood-CSF barrier function and to dysfunction in neurological diseases. J Neurol Sci 1994;122(2):189-203. doi: 10.1016/0022-510x(94)90298-4 [published Online First: 1994/04/01]

152. Hu Y, Sun J, Wu Z, et al. Predominant cerebral cytokine release syndrome in CD19directed chimeric antigen receptor-modified T cell therapy. J Hematol Oncol 2016;9(1):70. doi: 10.1186/s13045-016-0299-5 [published Online First: 2016/08/17]

153. Grupp SA, Kalos M, Barrett $D$, et al. Chimeric antigen receptor-modified T cells for acute lymphoid leukemia. N Eng/ J Med 2013;368(16):1509-18. doi:

10.1056/NEJMoa1215134 [published Online First: 2013/03/27]

154. Mueller KT, Maude SL, Porter DL, et al. Cellular kinetics of CTL019 in relapsed/refractory B-cell acute lymphoblastic leukemia and chronic lymphocytic leukemia. Blood 2017;130(21):2317-25. doi: 10.1182/blood-2017-06-786129 [published Online First: 2017/09/25]

155. McManus CM, Weidenheim K, Woodman SE, et al. Chemokine and chemokine-receptor expression in human glial elements: induction by the HIV protein, Tat, and chemokine autoregulation. Am J Pathol 2000;156(4):1441-53. doi: 10.1016/s00029440(10)65013-4 [published Online First: 2000/04/07]

156. Fuentes ME, Durham SK, Swerdel MR, et al. Controlled recruitment of monocytes and macrophages to specific organs through transgenic expression of monocyte chemoattractant protein-1. J Immunol 1995;155(12):5769-76. [published Online First: 1995/12/15]

157. Kochenderfer JN, Dudley ME, Kassim SH, et al. Chemotherapy-refractory diffuse large Bcell lymphoma and indolent B-cell malignancies can be effectively treated with autologous T cells expressing an anti-CD19 chimeric antigen receptor. J Clin Oncol 2015;33(6):540-9. doi: 10.1200/jco.2014.56.2025 [published Online First: 2014/08/27]

158. Hay KA, Turtle CJ. Chimeric Antigen Receptor (CAR) T Cells: Lessons Learned from Targeting of CD19 in B-Cell Malignancies. Drugs 2017;77(3):237-45. doi: 10.1007/s40265-017-0690-8 [published Online First: 2017/01/23]

159. Frisen L. Swelling of the optic nerve head: a staging scheme. J Neurol Neurosurg Psychiatry 1982;45(1):13-8. [published Online First: 1982/01/01]

160. Lowe KL, Mackall CL, Norry E, et al. Fludarabine and neurotoxicity in engineered T-cell therapy. Gene therapy 2018;25(3):176-91. doi: 10.1038/s41434-018-0019-6 [published Online First: 2018/05/24]

161. Frigault MJ, Dietrich J, Martinez-Lage M, et al. Tisagenlecleucel CAR T-cell therapy in secondary CNS lymphoma. Blood 2019;134(11):860-66. doi: 10.1182/blood.2019001694 [published Online First: 2019/07/20] 
162. Grupp S. Beginning the CAR T cell therapy revolution in the US and EU. Curr Res Trans/ Med 2018;66(2):62-64. doi: 10.1016/j.retram.2018.03.004 [published Online First: 2018/04/17]

163. Siddiqi TA, S. J.; Palomba, M. L et al. Correlation of patient characteristics and biomarkers with clinical outcomes of JCAR017 in R/R aggressive B-NHL (TRANSCEND NHL 001 study). American Society of Clinical Oncology: Journal of Clinical Oncology, 2018.

164. Karschnia P, Jordan JT, Forst DA, et al. Clinical presentation, management, and biomarkers of neurotoxicity after adoptive immunotherapy with CAR T cells. Blood 2019;133(20):2212-21. doi: 10.1182/blood-2018-12-893396 [published Online First: 2019/02/28]

165. Perica K, Curran KJ, Brentjens RJ, et al. Building a CAR Garage: Preparing for the Delivery of Commercial CAR T Cell Products at Memorial Sloan Kettering Cancer Center. Biol Blood Marrow Transplant 2018;24(6):1135-41. doi: 10.1016/j.bbmt.2018.02.018 [published Online First: 2018/03/03]

166. Gust J, Taraseviciute A, Turtle CJ. Neurotoxicity Associated with CD19-Targeted CAR-T Cell Therapies. CNS Drugs 2018;32(12):1091-101. doi: 10.1007/s40263-018-0582-9 [published Online First: 2018/11/06]

167. Locke FLN, S. S. Preliminary Results of Prophylactic Tocilizumab after Axicabtageneciloleucel (axi-cel; KTE-C19) Treatment for Patients with Refractory,Aggressive Non-Hodgkin Lymphoma (NHL). ASH: Blood Journal, 2017:1547.

168. Nellan A, McCully CML, Cruz Garcia R, et al. Improved CNS exposure to tocilizumab after cerebrospinal fluid compared to intravenous administration in rhesus macaques. Blood 2018;132(6):662-66. doi: 10.1182/blood-2018-05-846428 [published Online First: 2018/06/30]

169. Chen F, Teachey DT, Pequignot E, et al. Measuring IL-6 and sIL-6R in serum from patients treated with tocilizumab and/or siltuximab following CAR T cell therapy. J Immunol Methods 2016;434:1-8. doi: 10.1016/j.jim.2016.03.005 [published Online First: 2016/04/07]

170. Michinaga S, Koyama Y. Pathogenesis of brain edema and investigation into anti-edema drugs. Int J Mol Sci 2015;16(5):9949-75. doi: 10.3390/ijms16059949 [published Online First: 2015/05/06]

171. Shah B, Van H, Sender LS, et al. High Rates of Minimal Residual Disease-Negative (MRD-) Complete Responses (CR) in Adult and Pediatric and Patients With Relapsed/Refractory Acute Lymphoblastic Leukemia (R/R ALL) Treated With KTE-C19 (Anti-CD19 Chimeric Antigen Receptor CAR T Cells): Preliminary Results of the ZUMA3 and ZUMA-4 Trials. Blood 2016;128(22)

172. Bergsten E, Horne A, Arico M, et al. Confirmed efficacy of etoposide and dexamethasone in HLH treatment: long-term results of the cooperative HLH-2004 study. Blood 2017;130(25):2728-38. doi: 10.1182/blood-2017-06-788349 [published Online First: 2017/09/25]

173. Kumar B, Aleem S, Saleh H, et al. A Personalized Diagnostic and Treatment Approach for Macrophage Activation Syndrome and Secondary Hemophagocytic Lymphohistiocytosis in Adults. J Clin Immunol 2017;37(7):638-43. doi: 10.1007/s10875-017-0439-x [published Online First: 2017/09/06]

174. Kochenderfer JN, Dudley ME, Feldman SA, et al. B-cell depletion and remissions of malignancy along with cytokine-associated toxicity in a clinical trial of anti-CD19 
chimeric-antigen-receptor-transduced T cells. Blood 2012;119(12):2709-20. doi: 10.1182/blood-2011-10-384388 [published Online First: 2011/12/14]

175. Strati P, Nastoupil L, Fayad LE, et al. Safety of CAR T-cell therapy in patients with B-cell lymphoma and chronic hepatitis B or C virus infection. Blood 2019;133(26):2800-02. doi: 10.1182/blood.2019000888 [published Online First: 2019/05/19]

176. Fried S, Avigdor A, Bielorai B, et al. Early and late hematologic toxicity following CD19 CAR-T cells. Bone Marrow Transplant 2019;54(10):1643-50. doi: 10.1038/s41409-0190487-3 [published Online First: 2019/02/28]

177. Strati PN, S. S. Hematopoietic recovery and immune reconstitution after axi-cel CAR Tcell therapy in patients with relapsed/refractory large B-cell lymphoma. ASCO 2019: J Clin Oncol 37, 2019 (suppl; abstr 7545), 2019.

178. Finney HM, Akbar AN, Lawson AD. Activation of resting human primary T cells with chimeric receptors: costimulation from CD28, inducible costimulator, CD134, and CD137 in series with signals from the TCR zeta chain. Journal of immunology (Baltimore, Md : 1950) 2004;172(1):104-13. doi: 10.4049/jimmunol.172.1.104 [published Online First: 2003/12/23]

179. Imai C, Mihara K, Andreansky M, et al. Chimeric receptors with 4-1BB signaling capacity provoke potent cytotoxicity against acute lymphoblastic leukemia. Leukemia 2004;18(4):676-84. doi: 10.1038/sj.leu.2403302 [published Online First: 2004/02/13]

180. Mata M, Gerken C, Nguyen P, et al. Inducible Activation of MyD88 and CD40 in CAR T Cells Results in Controllable and Potent Antitumor Activity in Preclinical Solid Tumor Models. Cancer Discov 2017;7(11):1306-19. doi: 10.1158/2159-8290.Cd-17-0263 [published Online First: 2017/08/13]

181. Esensten JH, Helou YA, Chopra G, et al. CD28 Costimulation: From Mechanism to Therapy. Immunity 2016;44(5):973-88. doi: 10.1016/j.immuni.2016.04.020 [published Online First: 2016/05/19]

182. Zhong XS, Matsushita M, Plotkin J, et al. Chimeric antigen receptors combining 4-1BB and CD28 signaling domains augment PI3kinase/AKT/BCl-XL activation and CD8+ T cell-mediated tumor eradication. Mol Ther 2010;18(2):413-20. doi:

10.1038/mt.2009.210 [published Online First: 2009/09/24]

183. Condomines M, Arnason J, Benjamin R, et al. Tumor-Targeted Human T Cells Expressing CD28-Based Chimeric Antigen Receptors Circumvent CTLA-4 Inhibition. PLoS One 2015;10(6):e0130518. doi: 10.1371/journal.pone.0130518 [published Online First: 2015/06/26]

184. Kofler DM, Chmielewski M, Rappl G, et al. CD28 costimulation Impairs the efficacy of a redirected t-cell antitumor attack in the presence of regulatory t cells which can be overcome by preventing Lck activation. Mol Ther 2011;19(4):760-7. doi: 10.1038/mt.2011.9 [published Online First: 2011/02/18]

185. Zhao Z, Condomines M, van der Stegen SJC, et al. Structural Design of Engineered Costimulation Determines Tumor Rejection Kinetics and Persistence of CAR T Cells. Cancer cell 2015;28(4):415-28. doi: 10.1016/j.ccell.2015.09.004 [published Online First: 2015/10/16]

186. Chester C, Sanmamed MF, Wang J, et al. Immunotherapy targeting 4-1BB: mechanistic rationale, clinical results, and future strategies. Blood 2018;131(1):49-57. doi: 10.1182/blood-2017-06-741041 [published Online First: 2017/11/10]

187. Song DG, Ye Q, Carpenito C, et al. In vivo persistence, tumor localization, and antitumor activity of CAR-engineered T cells is enhanced by costimulatory signaling through 
CD137 (4-1BB). Cancer Res 2011;71(13):4617-27. doi: 10.1158/0008-5472.Can-110422 [published Online First: 2011/05/07]

188. Salter Al, Pont MJ, Riddell SR. Chimeric antigen receptor-modified T cells: CD19 and the road beyond. Blood 2018;131(24):2621-29. doi: 10.1182/blood-2018-01-785840 [published Online First: 2018/05/08]

189. Chapman NM, Bilal MY, Cruz-Orcutt N, et al. Distinct signaling pathways regulate TLR2 co-stimulatory function in human T cells. Cell Signal 2013;25(3):639-50. doi: 10.1016/j.cellsig.2012.11.026 [published Online First: 2012/12/12]

190. Ramos CA, Rouce R, Robertson CS, et al. In Vivo Fate and Activity of Second- versus Third-Generation CD19-Specific CAR-T Cells in B Cell Non-Hodgkin's Lymphomas. Mol Ther 2018;26(12):2727-37. doi: 10.1016/j.ymthe.2018.09.009 [published Online First: 2018/10/13]

191. Cheng Z, Wei R, Ma Q, et al. In Vivo Expansion and Antitumor Activity of Coinfused CD28- and 4-1BB-Engineered CAR-T Cells in Patients with B Cell Leukemia. Mol Ther 2018;26(4):976-85. doi: 10.1016/j.ymthe.2018.01.022 [published Online First: 2018/03/06]

192. Enblad G, Karlsson H, Gammelgard G, et al. A Phase I/Ila Trial Using CD19-Targeted Third-Generation CAR T Cells for Lymphoma and Leukemia. Clin Cancer Res 2018;24(24):6185-94. doi: 10.1158/1078-0432.Ccr-18-0426 [published Online First: 2018/08/12]

193. Boyiadzis MM, Dhodapkar MV, Brentjens RJ, et al. Chimeric antigen receptor (CAR) T therapies for the treatment of hematologic malignancies: clinical perspective and significance. J Immunother Cancer 2018;6(1):137. doi: 10.1186/s40425-018-0460-5 [published Online First: 2018/12/06]

194. Hartmann J, Schussler-Lenz M, Bondanza A, et al. Clinical development of CAR T cellschallenges and opportunities in translating innovative treatment concepts. EMBO Mol Med 2017;9(9):1183-97. doi: 10.15252/emmm.201607485

195. Rosenberg SA, Lotze MT, Muul LM, et al. Observations on the systemic administration of autologous lymphokine-activated killer cells and recombinant interleukin-2 to patients with metastatic cancer. N Engl J Med 1985;313(23):1485-92. doi: 10.1056/NEJM198512053132327 [published Online First: 1985/12/05]

196. Rosenberg SA, Packard BS, Aebersold PM, et al. Use of Tumor-Infiltrating Lymphocytes and Interleukin-2 in the Immunotherapy of Patients with Metastatic Melanoma - a Preliminary-Report. New Engl J Med 1988;319(25):1676-80. doi: Doi 10.1056/Nejm198812223192527

197. Kuwana Y, Asakura Y, Utsunomiya N, et al. Expression of chimeric receptor composed of immunoglobulin-derived $\mathrm{V}$ regions and T-cell receptor-derived $\mathrm{C}$ regions. Biochemical and biophysical research communications 1987;149(3):960-8. [published Online First: 1987/12/31]

198. Maher J, Brentjens RJ, Gunset G, et al. Human T-lymphocyte cytotoxicity and proliferation directed by a single chimeric TCR zeta/CD28 receptor. Nat Biotechnol 2002;20(1):70-75. doi: DOI 10.1038/nbt0102-70

199. Finney HM, Lawson AD, Bebbington $C R$, et al. Chimeric receptors providing both primary and costimulatory signaling in T cells from a single gene product. Journal of immunology (Baltimore, Md : 1950) 1998;161(6):2791-7. [published Online First: 1998/09/22] 
200. Weinkove R, George P, Dasyam N, et al. Selecting costimulatory domains for chimeric antigen receptors: functional and clinical considerations. Clin Trans/ Immunology 2019;8(5):e1049. doi: 10.1002/cti2.1049 [published Online First: 2019/05/22]

201. Tokarew N, Ogonek J, Endres S, et al. Teaching an old dog new tricks: next-generation CAR T cells. Br J Cancer 2019;120(1):26-37. doi: 10.1038/s41416-018-0325-1 [published Online First: 2018/11/11]

202. Vormittag P, Gunn R, Ghorashian S, et al. A guide to manufacturing CAR T cell therapies. Curr Opin Biotech 2018;53:164-81. doi: 10.1016/j.copbio.2018.01.025

203. Roddie C, O'Reilly M, Dias Alves Pinto J, et al. Manufacturing chimeric antigen receptor T cells: issues and challenges. Cytotherapy 2019;21(3):327-40. doi: 10.1016/j.jcyt.2018.11.009 [published Online First: 2019/01/28]

204. Wang X, Riviere I. Clinical manufacturing of CAR T cells: foundation of a promising therapy. Mol Ther Oncolytics 2016;3:16015. doi: 10.1038/mto.2016.15 [published Online First: 2016/06/28]

205. Kalos M, Levine BL, Porter DL, et al. T cells with chimeric antigen receptors have potent antitumor effects and can establish memory in patients with advanced leukemia. Sci Trans/ Med 2011;3(95):95ra73. doi: 10.1126/scitransImed.3002842 [published Online First: 2011/08/13]

206. Kochenderfer JN, Somerville RPT, Lu TY, et al. Long-Duration Complete Remissions of Diffuse Large B Cell Lymphoma after Anti-CD19 Chimeric Antigen Receptor T Cell Therapy. Mol Ther 2017;25(10):2245-53. doi: 10.1016/j.ymthe.2017.07.004

207. Ritchie DS, Neeson PJ, Khot A, et al. Persistence and Efficacy of Second Generation CAR T Cell Against the LeY Antigen in Acute Myeloid Leukemia. Mol Ther 2013;21(11):212229. doi: 10.1038/mt.2013.154

208. Jena B, Maiti S, Huls H, et al. Chimeric antigen receptor (CAR)-specific monoclonal antibody to detect CD19-specific T cells in clinical trials. PLoS One 2013;8(3):e57838. doi: 10.1371/journal.pone.0057838 [published Online First: 2013/03/08]

209. Zheng Z, Chinnasamy N, Morgan RA. Protein L: a novel reagent for the detection of chimeric antigen receptor (CAR) expression by flow cytometry. J Trans/ Med 2012;10:29. doi: 10.1186/1479-5876-10-29 [published Online First: 2012/02/15]

210. De Oliveira SN, Wang J, Ryan C, et al. A CD19/Fc fusion protein for detection of antiCD19 chimeric antigen receptors. J Trans/ Med 2013;11:23. doi: 10.1186/1479-587611-23 [published Online First: 2013/01/31]

211. Wang X, Chang WC, Wong CW, et al. A transgene-encoded cell surface polypeptide for selection, in vivo tracking, and ablation of engineered cells. Blood 2011;118(5):125563. doi: 10.1182/blood-2011-02-337360 [published Online First: 2011/06/10]

212. O'Rourke DM, Nasrallah MP, Desai A, et al. A single dose of peripherally infused EGFRVIII-directed CAR T cells mediates antigen loss and induces adaptive resistance in patients with recurrent glioblastoma. Sci Trans/ Med 2017;9(399) doi: 10.1126/scitranslmed.aaa0984 [published Online First: 2017/07/21]

213. Weist MR, Starr R, Aguilar B, et al. PET of Adoptively Transferred Chimeric Antigen Receptor T Cells with (89)Zr-Oxine. J Nucl Med 2018;59(10):1531-37. doi: 10.2967/jnumed.117.206714 [published Online First: 2018/05/08]

214. Meier R, Golovko D, Tavri S, et al. Depicting adoptive immunotherapy for prostate cancer in an animal model with magnetic resonance imaging. Magn Reson Med 2011;65(3):756-63. doi: 10.1002/mrm.22652 [published Online First: 2010/10/12] 
215. Minn I, Huss DJ, Ahn HH, et al. Imaging CAR T cell therapy with PSMA-targeted positron emission tomography. Sci Adv 2019;5(7):eaaw5096. doi: 10.1126/sciadv.aaw5096 [published Online First: 2019/07/10]

216. Keu KV, Witney TH, Yaghoubi S, et al. Reporter gene imaging of targeted T cell immunotherapy in recurrent glioma. Sci Transl Med 2017;9(373) doi: 10.1126/scitransImed.aag2196 [published Online First: 2017/01/20]

217. Wallace PK, Palmer LD, Perry-Lalley D, et al. Mechanisms of adoptive immunotherapy: improved methods for in vivo tracking of tumor-infiltrating lymphocytes and lymphokine-activated killer cells. Cancer Res 1993;53(10 Suppl):2358-67. [published Online First: 1993/05/15]

218. Park JH, Geyer MB, Brentjens RJ. CD19-targeted CAR T-cell therapeutics for hematologic malignancies: interpreting clinical outcomes to date. Blood 2016;127(26):3312-20. doi: 10.1182/blood-2016-02-629063 [published Online First: 2016/05/22]

219. Hirayama AV, Gauthier J, Hay KA, et al. The response to lymphodepletion impacts PFS in patients with aggressive non-Hodgkin lymphoma treated with CD19 CAR T cells. Blood 2019;133(17):1876-87. doi: 10.1182/blood-2018-11-887067 [published Online First: 2019/02/21]

220. Gattinoni L, Finkelstein SE, Klebanoff CA, et al. Removal of homeostatic cytokine sinks by lymphodepletion enhances the efficacy of adoptively transferred tumor-specific CD8+ T cells. J Exp Med 2005;202(7):907-12. doi: 10.1084/jem.20050732 [published Online First: 2005/10/06]

221. Rosenberg SA, Restifo NP, Yang JC, et al. Adoptive cell transfer: a clinical path to effective cancer immunotherapy. Nat Rev Cancer 2008;8(4):299-308. doi: 10.1038/nrc2355 [published Online First: 2008/03/21]

222. Kochenderfer JN, Yu Z, Frasheri D, et al. Adoptive transfer of syngeneic T cells transduced with a chimeric antigen receptor that recognizes murine CD19 can eradicate lymphoma and normal B cells. Blood 2010;116(19):3875-86. doi: 10.1182/blood-2010-01-265041 [published Online First: 2010/07/16]

223. Susanibar Adaniya SP, Cohen AD, Garfall AL. Chimeric antigen receptor T cell immunotherapy for multiple myeloma: A review of current data and potential clinical applications. American journal of hematology 2019;94(S1):S28-s33. doi: 10.1002/ajh.25428 [published Online First: 2019/02/08]

224. Brudno JN, Maric I, Hartman SD, et al. T Cells Genetically Modified to Express an Anti-BCell Maturation Antigen Chimeric Antigen Receptor Cause Remissions of PoorPrognosis Relapsed Multiple Myeloma. Journal of clinical oncology : official journal of the American Society of Clinical Oncology 2018;36(22):2267-80. doi:

10.1200/jco.2018.77.8084 [published Online First: 2018/05/31]

225. Mishra A, Sullivan L, Caligiuri MA. Molecular pathways: interleukin-15 signaling in health and in cancer. Clinical cancer research : an official journal of the American Association for Cancer Research 2014;20(8):2044-50. doi: 10.1158/1078-0432.Ccr-12-3603 [published Online First: 2014/04/17]

226. USFDA. YESCARTA (axicabtagene ciloleucel): U.S. Food \& Drug Administration; 2018 [Available from: https://www.fda.gov/vaccines-blood-biologics/cellular-gene-therapyproducts/yescarta-axicabtagene-ciloleucel.

227. USFDA. KYMRIAH (tisagenlecleucel) U.S. Food \& Drug Administration2019 [Available from: https://www.fda.gov/vaccines-blood-biologics/cellular-gene-therapyproducts/kymriah-tisagenlecleucel. 
228. Liu B, Yan L, Zhou M. Target selection of CAR T cell therapy in accordance with the TME for solid tumors. Am J Cancer Res 2019;9(2):228-41. [published Online First: 2019/03/25]

229. Cheadle EJ, Hawkins RE, Batha $\mathrm{H}$, et al. Natural expression of the CD19 antigen impacts the long-term engraftment but not antitumor activity of CD19-specific engineered T cells. Journal of immunology (Baltimore, Md : 1950) 2010;184(4):1885-96. doi: 10.4049/jimmunol.0901440 [published Online First: 2010/01/22]

230. Guedan S, Calderon H, Posey AD, Jr., et al. Engineering and Design of Chimeric Antigen Receptors. Mol Ther Methods Clin Dev 2019;12:145-56. doi: 10.1016/j.omtm.2018.12.009 [published Online First: 2019/01/23]

231. Grigor EJM, Fergusson D, Kekre N, et al. Risks and Benefits of Chimeric Antigen Receptor T-Cell (CAR-T) Therapy in Cancer: A Systematic Review and Meta-Analysis. Transfus Med Rev 2019;33(2):98-110. doi: 10.1016/j.tmrv.2019.01.005 [published Online First: 2019/04/06]

232. Mardiana S, Solomon BJ, Darcy PK, et al. Supercharging adoptive T cell therapy to overcome solid tumor-induced immunosuppression. Sci Trans/ Med 2019;11(495) doi: 10.1126/scitransImed.aaw2293 [published Online First: 2019/06/07]

233. Kawalekar OU, O'Connor RS, Fraietta JA, et al. Distinct Signaling of Coreceptors Regulates Specific Metabolism Pathways and Impacts Memory Development in CAR T Cells. Immunity 2016;44(2):380-90. doi: 10.1016/j.immuni.2016.01.021 [published Online First: 2016/02/18]

234. Ramos CA, Rouce R, Robertson CS, et al. In Vivo Fate and Activity of Second- versus Third-Generation CD19-Specific CAR-T Cells in B Cell Non-Hodgkin's Lymphomas. Mol Ther 2018 doi: 10.1016/j.ymthe.2018.09.009 [published Online First: 2018/10/13]

235. Sommermeyer D, Hudecek M, Kosasih PL, et al. Chimeric antigen receptor-modified $T$ cells derived from defined CD8+ and CD4+ subsets confer superior antitumor reactivity in vivo. Leukemia 2016;30(2):492-500. doi: 10.1038/leu.2015.247 [published Online First: 2015/09/16]

236. Rouce RH, Heslop HE. Equal opportunity CAR T cells. Blood 2017;129(25):3275-77. doi: 10.1182/blood-2017-04-779983 [published Online First: 2017/06/24]

237. McLellan AD, Ali Hosseini Rad SM. Chimeric antigen receptor T cell persistence and memory cell formation. Immunol Cell Biol 2019;97(7):664-74. doi: 10.1111/imcb.12254 [published Online First: 2019/04/23]

238. Klebanoff CA, Finkelstein SE, Surman DR, et al. IL-15 enhances the in vivo antitumor activity of tumor-reactive CD8+ T cells. Proceedings of the National Academy of Sciences of the United States of America 2004;101(7):1969-74. doi: 10.1073/pnas.0307298101 [published Online First: 2004/02/06]

239. Xu Y, Zhang $M$, Ramos $C A$, et al. Closely related T-memory stem cells correlate with in vivo expansion of CAR.CD19-T cells and are preserved by IL-7 and IL-15. Blood 2014;123(24):3750-9. doi: 10.1182/blood-2014-01-552174 [published Online First: 2014/05/02]

240. Singh N, Perazzelli J, Grupp SA, et al. Early memory phenotypes drive T cell proliferation in patients with pediatric malignancies. Sci Trans/ Med 2016;8(320):320ra3. doi: 10.1126/scitranslmed.aad5222 [published Online First: 2016/01/08]

241. Hinrichs CS, Spolski R, Paulos CM, et al. IL-2 and IL-21 confer opposing differentiation programs to CD8+ T cells for adoptive immunotherapy. Blood 2008;111(11):5326-33. doi: 10.1182/blood-2007-09-113050 [published Online First: 2008/02/16] 
242. Singh H, Figliola MJ, Dawson MJ, et al. Reprogramming CD19-specific T cells with IL-21 signaling can improve adoptive immunotherapy of B-lineage malignancies. Cancer Res 2011;71(10):3516-27. doi: 10.1158/0008-5472.CAN-10-3843 [published Online First: 2011/05/12]

243. Herold MJ, McPherson KG, Reichardt HM. Glucocorticoids in T cell apoptosis and function. Cell Mol Life Sci 2006;63(1):60-72. doi: 10.1007/s00018-005-5390-y [published Online First: 2005/11/30]

244. Hill BT, Roberts ZJ, Xue A, et al. Rapid tumor regression from PD-1 inhibition after antiCD19 chimeric antigen receptor T-cell therapy in refractory diffuse large B-cell Iymphoma. Bone Marrow Transplant 2019 doi: 10.1038/s41409-019-0657-3 [published Online First: 2019/09/01]

245. Schuster SJ, Svoboda J, Chong EA, et al. Chimeric Antigen Receptor T Cells in Refractory B-Cell Lymphomas. N Engl J Med 2017;377(26):2545-54. doi: 10.1056/NEJMoa1708566

246. The effect of pembrolizumab in combination with CD19-targeted chimeric antigen receptor (CAR) T cells in relapsed acute lymphoblastic leukemia (ALL). American Society of Clinical Oncology 2017. Journal of Clincal Oncology.

247. Fesnak AD. The Challenge of Variability in Chimeric Antigen Receptor T Cell Manufacturing. Regenerative Engineering and Translational Medicine 2019 doi: 10.1007/s40883-019-00124-3

248. Tuazon SA, Li A, Gooley T, et al. Factors affecting lymphocyte collection efficiency for the manufacture of chimeric antigen receptor T cells in adults with B-cell malignancies. Transfusion 2019;59(5):1773-80. doi: 10.1111/trf.15178 [published Online First: 2019/02/08\{Tuazon, 2019 \#479\}]

249. Scarfo I, Maus MV. Current approaches to increase CAR T cell potency in solid tumors: targeting the tumor microenvironment. J Immunother Cancer 2017;5:28. doi: 10.1186/s40425-017-0230-9 [published Online First: 2017/03/24]

250. Depil S, Duchateau P, Grupp SA, et al. 'Off-the-shelf' allogeneic CAR T cells: development and challenges. Nat Rev Drug Discov 2020 doi: 10.1038/s41573-019-0051-2 [published Online First: 2020/01/05]

251. Salmikangas P, Kinsella N, Chamberlain P. Chimeric Antigen Receptor T-Cells (CAR TCells) for Cancer Immunotherapy - Moving Target for Industry? Pharmaceutical research 2018;35(8):152. doi: 10.1007/s11095-018-2436-z [published Online First: 2018/06/02]

252. Ali SA, Shi V, Maric I, et al. T cells expressing an anti-B-cell maturation antigen chimeric antigen receptor cause remissions of multiple myeloma. Blood 2016;128(13):1688700. doi: 10.1182/blood-2016-04-711903 [published Online First: 2016/07/15]

253. Gardner RA, Finney O, Annesley C, et al. Intent-to-treat leukemia remission by CD19 CAR $T$ cells of defined formulation and dose in children and young adults. Blood 2017;129(25):3322-31. doi: 10.1182/blood-2017-02-769208 [published Online First: 2017/04/15]

254. Awasthi RTSCea. Clinical pharmacology of tisagenlecleucel (CTLO19) in patients with relapsed/refractory $(r / r)$ diffuse large B-cell lymphoma (DLBCL). American Association of Cancer Research Annual Meeting: AACR Publications, 2018.

255. Raje N, Berdeja J, Lin Y, et al. Anti-BCMA CAR T-Cell Therapy bb2121 in Relapsed or Refractory Multiple Myeloma. N Engl J Med 2019;380(18):1726-37. doi: 10.1056/NEJMoa1817226 [published Online First: 2019/05/03] 
256. Frey NV. Chimeric antigen receptor T cells for acute lymphoblastic leukemia. American journal of hematology 2019;94(S1):S24-s27. doi: 10.1002/ajh.25442 [published Online First: 2019/02/21]

257. An updated analysis of JULIET, a global pivotal Phase 2 trial of tisagenlecleucel in adult patients with relapsed or refractory $(r / r)$ diffuse large b-cell lymphoma (DLBCL). The 23rd Congress of EHA; 2018; Stockholm.

258. Zola H, MacArdle PJ, Bradford T, et al. Preparation and characterization of a chimeric CD19 monoclonal antibody. Immunol Cell Biol 1991;69 ( Pt 6):411-22. doi: 10.1038/icb.1991.58 [published Online First: 1991/12/01]

259. Qian L, Li D, Ma L, et al. The novel anti-CD19 chimeric antigen receptors with humanized scFv (single-chain variable fragment) trigger leukemia cell killing. Cell Immunol 2016;304-305:49-54. doi: 10.1016/j.cellimm.2016.03.003 [published Online First: 2016/03/22]

260. Storer BE. Design and analysis of phase I clinical trials. Biometrics 1989;45(3):925-37. [published Online First: 1989/09/01]

261. Schubert ML, Schmitt A, Sellner L, et al. Treatment of patients with relapsed or refractory CD19+ lymphoid disease with T lymphocytes transduced by RVSFG.CD19.CD28.4-1BBzeta retroviral vector: a unicentre phase I/II clinical trial protocol. BMJ open 2019;9(5):e026644. doi: 10.1136/bmjopen-2018-026644 [published Online First: 2019/05/22]

262. Seimetz D, Heller K, Richter J. Approval of First CAR-Ts: Have we Solved all Hurdles for ATMPs? Cell Med 2019;11:1-16. [published Online First: 2019 Jan 22]

263. Li DH, Whitmore JB, Guo W, et al. Toxicity and Efficacy Probability Interval Design for Phase I Adoptive Cell Therapy Dose-Finding Clinical Trials. Clinical cancer research : an official journal of the American Association for Cancer Research 2017;23(1):13-20. doi: 10.1158/1078-0432.Ccr-16-1125 [published Online First: 2016/10/16]

264. Wages NA, Chiuzan C, Panageas KS. Design considerations for early-phase clinical trials of immune-oncology agents. J Immunother Cancer 2018;6(1):81. doi: 10.1186/s40425-018-0389-8 [published Online First: 2018/08/24]

265. Ananthakrishnan R, Green S, Li D, et al. Extensions of the mTPI and TEQR designs to include non-monotone efficacy in addition to toxicity for optimal dose determination for early phase immunotherapy oncology trials. Contemporary clinical trials communications 2018;10:62-76. doi: 10.1016/j.conctc.2018.01.006 [published Online First: 2018/04/27]

266. Ananthakrishnan R, Green S, Li D, et al. 2D (2 Dimensional) TEQR design for Determining the optimal Dose for safety and efficacy. Contemporary clinical trials communications 2019;16:100461. doi: 10.1016/j.conctc.2019.100461 [published Online First: 2019/12/05]

267. Chiuzan C, Garrett-Mayer E, Nishimura M. An adaptive dose-finding design based on both safety and immunologic responses in cancer clinical trials. Statistics in biopharmaceutical research 2018;10(3):185-95. doi: 10.1080/19466315.2018.1462727 [published Online First: 2018/12/14]

268. Watanabe K, Kuramitsu S, Posey AD, Jr., et al. Expanding the Therapeutic Window for CAR T Cell Therapy in Solid Tumors: The Knowns and Unknowns of CAR T Cell Biology. Front Immunol 2018;9:2486. doi: 10.3389/fimmu.2018.02486 [published Online First: 2018/11/13] 
269. Hay AE, Cheung MC. CAR T-cells: costs, comparisons, and commentary. J Med Econ 2019;22(7):613-15. doi: 10.1080/13696998.2019.1582059 [published Online First: 2019/02/13]

270. Lin JK, Muffly LS, Spinner MA, et al. Cost Effectiveness of Chimeric Antigen Receptor TCell Therapy in Multiply Relapsed or Refractory Adult Large B-Cell Lymphoma. Journal of clinical oncology : official journal of the American Society of Clinical Oncology 2019;37(24):2105-19. doi: 10.1200/jco.18.02079 [published Online First: 2019/06/04]

271. Qasim W, Zhan H, Samarasinghe S, et al. Molecular remission of infant B-ALL after infusion of universal TALEN gene-edited CAR T cells. Sci Trans/ Med 2017;9(374) doi: 10.1126/scitransImed.aaj2013 [published Online First: 2017/01/27]

272. Liu E, Marin D, Banerjee P, et al. Use of CAR-Transduced Natural Killer Cells in CD19Positive Lymphoid Tumors. N Engl J Med 2020;382(6):545-53. doi: 10.1056/NEJMoa1910607 [published Online First: 2020/02/06]

273. Xu X, Huang W, Heczey A, et al. NKT Cells Coexpressing a GD2-Specific Chimeric Antigen Receptor and IL15 Show Enhanced In Vivo Persistence and Antitumor Activity against Neuroblastoma. Clinical cancer research : an official journal of the American Association for Cancer Research 2019;25(23):7126-38. doi: 10.1158/1078-0432.CCR19-0421 [published Online First: 2019/09/06]

274. Li Y, Hermanson DL, Moriarity BS, et al. Human iPSC-Derived Natural Killer Cells Engineered with Chimeric Antigen Receptors Enhance Anti-tumor Activity. Cell Stem Cell 2018;23(2):181-92 e5. doi: 10.1016/j.stem.2018.06.002 [published Online First: 2018/08/08]

275. Oldham RAA, Medin JA. Practical considerations for chimeric antigen receptor design and delivery. Expert Opin Biol Ther 2017;17(8):961-78. doi: 10.1080/14712598.2017.1339687 [published Online First: 2017/06/07]

276. Lynch M. Novartis-NICE deal makes Kymriah available to UK patients. Biopharmareportercom 2019 6th February 2019.

277. George P, Dasyam N, Giunti G, et al. Third-generation anti-CD19 chimeric antigen receptor T-cells incorporating a TLR2 domain for relapsed or refractory B-cell Iymphoma: a phase I clinical trial protocol (ENABLE). BMJ Open 2020;10(2):e034629. doi: 10.1136/bmjopen-2019-034629 [published Online First: 2020/02/12]

278. Levine BL, Miskin J, Wonnacott K, et al. Global Manufacturing of CAR T Cell Therapy. Mol Ther Methods Clin Dev 2017;4:92-101. doi: 10.1016/j.omtm.2016.12.006 [published Online First: 2017/03/28]

279. Allen ES, Stroncek DF, Ren J, et al. Autologous lymphapheresis for the production of chimeric antigen receptor T cells. Transfusion 2017;57(5):1133-41. doi:

10.1111/trf.14003 [published Online First: 2017/02/27]

280. Health Mo. Medsafe Licensed Medicine Manufacturing Sites 2020 [

281. Kiki I. What is the role of apheresis technology in stem cell transplantation? Transfus Apher Sci 2017;56(6):788-94. doi: 10.1016/j.transci.2017.11.007 [published Online First: 2017/11/23]

282. Gasser O, Sharples KJ, Barrow C, et al. A phase I vaccination study with dendritic cells loaded with NY-ESO-1 and alpha-galactosylceramide: induction of polyfunctional T cells in high-risk melanoma patients. Cancer Immunol Immunother 2018;67(2):285-98. doi: 10.1007/s00262-017-2085-9 [published Online First: 2017/11/03]

283. Teng AM, Atkinson J, Disney G, et al. Ethnic inequalities in cancer incidence and mortality: census-linked cohort studies with 87 million years of person-time follow- 
up. BMC Cancer 2016;16(1):755. doi: 10.1186/s12885-016-2781-4 [published Online First: 2016/09/28]

284. Disney G, Teng A, Atkinson J, et al. Changing ethnic inequalities in mortality in New Zealand over 30 years: linked cohort studies with 68.9 million person-years of followup. Popul Health Metr 2017;15:15. doi: 10.1186/s12963-017-0132-6 [published Online First: 2017/04/30]

285. Committee NEA. National Ethical Standards

for Health and Disability Research and Quality Improvement. Wellington. In: Health Mo, ed., 2019.

286. Simmonds. A Framework for Māori Review of Research in District Heath Boards: Auckland and WaitematāDistrict Health Boards and Capital and Coast District Health Board. Wellington, New Zealand, 2015.

287. Berger I, Ahmad A, Bansal A, et al. Global Distribution of Businesses Marketing Stem Cell-Based Interventions. Cell Stem Cell 2016;19(2):158-62. doi: 10.1016/j.stem.2016.07.015 [published Online First: 2016/08/06]

288. Lipworth W, Axler R. Towards a bioethics of innovation. J Med Ethics 2016;42(7):445-9. doi: 10.1136/medethics-2015-103048 [published Online First: 2016/03/27]

289. Switzer GE, Bruce JG, Myaskovsky L, et al. Race and ethnicity in decisions about unrelated hematopoietic stem cell donation. Blood 2013;121(8):1469-76. doi: 10.1182/blood-2012-06-437343 [published Online First: 2012/12/22]

290. Dzau VJ, Balatbat CA. Health and societal implications of medical and technological advances. Science translational medicine 2018;10(463) doi: 10.1126/scitransImed.aau4778 [published Online First: 2018/10/20]

291. Agency PM. New Zealand Pharmaceutical Schedule. In: PHARMAC, ed.: New Zealand Government, 2019.

292. Taylor L, Rodriguez ES, Reese A, et al. Building a Program: Implications for Infrastructure, Nursing Education, and Training for CAR T-Cell Therapy. Clin I Oncol Nurs 2019;23(2):20-26. doi: 10.1188/19.Cjon.S1.20-26 [published Online First: 2019/03/19]

293. Services. USDoHaH. Long term follow-up after administration of human gene therapy products. In: Services. USDoHaH, ed., July 2018.

294. Pasquini MCP, M-A. Real World Data on CAR T-Cell Recipients: Are We There Yet? The Hematologist 2019;16(2)

295. Nivison-Smith I, Bardy P, Dodds AJ, et al. A Review of Hematopoietic Cell Transplantation in Australia and New Zealand, 2005 to 2013. Biology of blood and marrow transplantation : journal of the American Society for Blood and Marrow Transplantation 2016;22(2):284-91. doi: 10.1016/j.bbmt.2015.09.009 [published Online First: 2015/09/20]

296. Bachanova V, Bishop MR, Dahi P, et al. CAR T Cell Therapy During the COVID-19 Pandemic. Biol Blood Marrow Transplant 2020 doi: 10.1016/j.bbmt.2020.04.008 [published Online First: 2020/04/17]

297. Recher C, Coiffier B, Haioun C, et al. Intensified chemotherapy with ACVBP plus rituximab versus standard $\mathrm{CHOP}$ plus rituximab for the treatment of diffuse large $\mathrm{B}$ cell lymphoma (LNH03-2B): an open-label randomised phase 3 trial. Lancet 2011;378(9806):1858-67. doi: 10.1016/S0140-6736(11)61040-4

298. Benmebarek MR, Karches CH, Cadilha BL, et al. Killing Mechanisms of Chimeric Antigen Receptor (CAR) T Cells. International journal of molecular sciences 2019;20(6) doi: 10.3390/ijms20061283 [published Online First: 2019/03/17] 
299. Schuster SJ, Bishop MR, Tam CS, et al. Tisagenlecleucel in Adult Relapsed or Refractory Diffuse Large B-Cell Lymphoma. N Engl J Med 2019;380(1):45-56. doi: 10.1056/NEJMoa1804980 [published Online First: 2018/12/07]

300. Karlsson H, Svensson E, Gigg C, et al. Evaluation of Intracellular Signaling Downstream Chimeric Antigen Receptors. PLoS One 2015;10(12):e0144787. doi: 10.1371/journal.pone.0144787 [published Online First: 2015/12/25]

301. Carpenito C, Milone MC, Hassan R, et al. Control of large, established tumor xenografts with genetically retargeted human T cells containing CD28 and CD137 domains. Proceedings of the National Academy of Sciences of the United States of America 2009;106(9):3360-5. doi: 10.1073/pnas.0813101106 [published Online First: 2009/02/13]

302. Komai-Koma M, Jones L, Ogg GS, et al. TLR2 is expressed on activated T cells as a costimulatory receptor. Proceedings of the National Academy of Sciences of the United States of America 2004;101(9):3029-34. doi: 10.1073/pnas.0400171101 [published Online First: 2004/02/26]

303. Rahman AH, Taylor DK, Turka LA. The contribution of direct TLR signaling to T cell responses. Immunologic research 2009;45(1):25-36. doi: 10.1007/s12026-009-8113-x [published Online First: 2009/07/15]

304. Cottalorda A, Mercier BC, Mbitikon-Kobo FM, et al. TLR2 engagement on memory $\mathrm{CD} 8(+) \mathrm{T}$ cells improves their cytokine-mediated proliferation and IFN-gamma secretion in the absence of Ag. European journal of immunology 2009;39(10):267381. doi: 10.1002/eji.200939627 [published Online First: 2009/07/28]

305. Mercier BC, Cottalorda A, Coupet CA, et al. TLR2 engagement on CD8 T cells enables generation of functional memory cells in response to a suboptimal TCR signal. Journal of immunology (Baltimore, Md : 1950) 2009;182(4):1860-7. doi: 10.4049/jimmunol.0801167 [published Online First: 2009/02/10]

306. Cheson BD, Fisher RI, Barrington SF, et al. Recommendations for initial evaluation, staging, and response assessment of Hodgkin and non-Hodgkin lymphoma: the Lugano classification. J Clin Oncol 2014;32(27):3059-68. doi: 10.1200/jco.2013.54.8800 [published Online First: 2014/08/13]

307. Neelapu SS, Tummala S, Kebriaei $P$, et al. Toxicity management after chimeric antigen receptor T cell therapy: one size does not fit 'ALL'. Nat Rev Clin Oncol 2018 doi: 10.1038/nrclinonc. 2018.20

308. Declaration of Helsinki. In: Assembly tWMAG, ed. Edinburgh, Scotland: World Medical Association, Inc, 2008.

309. Medsafe. Guideline on the Regulation of Therapeutic Produts in New Zealand. Part 11: Clinical trials - regulatory and good clinical practice requirements. In: Authority NZMaMDS, ed. 2.0 ed. Ministry of Health, 2018.

310. Third-Generation CAR T Cells Targeting CD19 Are Associated with an Excellent Safety Profile and Might Improve Persistence of CAR T Cells in Treated Patients. ASH 2019; 2019; Orlando, Florida. Blood.

311. Leslie LA, Feldman TA, McNeill A, et al. Contemporary management of nodal and primary splenic marginal zone lymphoma. Expert Rev Hematol 2019;12(12):1011-22. doi: 10.1080/17474086.2020.1681962 [published Online First: 2019/10/18]

312. Stroncek DF, Ren J, Lee DW, et al. Myeloid cells in peripheral blood mononuclear cell concentrates inhibit the expansion of chimeric antigen receptor T cells. Cytotherapy 
2016;18(7):893-901. doi: 10.1016/j.jcyt.2016.04.003 [published Online First:

2016/05/24]

313. Kotsakis A, Harasymczuk M, Schilling B, et al. Myeloid-derived suppressor cell measurements in fresh and cryopreserved blood samples. J Immunol Methods 2012;381(1-2):14-22. doi: 10.1016/j.jim.2012.04.004 [published Online First: 2012/04/24]

314. Downs D. A Mild Touch of the Cancer2019.

315. Macandrew R. The million-dollar campaign to get a cutting-edge cancer treatment to kiwis. Stuff 2018.

316. The Lancet Infectious D. COVID-19, a pandemic or not? Lancet Infect Dis 2020;20(4):383. doi: 10.1016/s1473-3099(20)30180-8 [published Online First: 2020/03/18]

317. Weinkove RM, Z.; Adler, J et al. Managing haematology and oncology patients during the COVID-19 pandemic: interim consensus guidance. Medical Journal of Australia 2020

318. Loo-Yong-Kee SH, C.; Ramsaroop, D et al. Development of a semi-automated closed CAR-T manufacturing process. gelifesciences.com/celltherapy: Center for Commercialisation of Regenerative Medicine, 2019.

319. Zhu F, Shah N, Xu H, et al. Closed-system manufacturing of CD19 and dual-targeted CD20/19 chimeric antigen receptor $T$ cells using the CliniMACS Prodigy device at an academic medical center. Cytotherapy 2018;20(3):394-406. doi: 10.1016/j.jcyt.2017.09.005 [published Online First: 2017/12/31]

320. Oberschmidt O, Morgan M, Huppert V, et al. Development of Automated Separation, Expansion, and Quality Control Protocols for Clinical-Scale Manufacturing of Primary Human NK Cells and Alpharetroviral Chimeric Antigen Receptor Engineering. Hum Gene Ther Methods 2019;30(3):102-20. doi: 10.1089/hgtb.2019.039 [published Online First: 2019/04/19]

321. Wilson MH. Consider Changing the Horse for Your CAR-T? Mol Ther 2018;26(8):1873-74. doi: 10.1016/j.ymthe.2018.06.018 [published Online First: 2018/08/07]

322. Hudecek M, Ivics Z. Non-viral therapeutic cell engineering with the Sleeping Beauty transposon system. Curr Opin Genet Dev 2018;52:100-08. doi: 10.1016/j.gde.2018.06.003 [published Online First: 2018/06/30]

323. Hackett PB, Largaespada DA, Switzer KC, et al. Evaluating risks of insertional mutagenesis by DNA transposons in gene therapy. Trans/ Res 2013;161(4):265-83. doi: 10.1016/j.trsl.2012.12.005 [published Online First: 2013/01/15]

324. Friedrich MJ, Bronner IF, Liu P, et al. PiggyBac Transposon-Based Insertional Mutagenesis in Mice. Methods Mol Biol 2019;1907:171-83. doi: 10.1007/978-1-49398967-6_14 [published Online First: 2018/12/14]

325. Zeiser R, Blazar BR. Acute Graft-versus-Host Disease - Biologic Process, Prevention, and Therapy. N Engl J Med 2017;377(22):2167-79. doi: 10.1056/NEJMra1609337 [published Online First: 2017/11/25]

326. Felix NJ, Allen PM. Specificity of T-cell alloreactivity. Nat Rev Immunol 2007;7(12):94253. doi: 10.1038/nri2200 [published Online First: 2007/11/17]

327. Melenhorst JJ, Leen AM, Bollard CM, et al. Allogeneic virus-specific T cells with HLA alloreactivity do not produce GVHD in human subjects. Blood 2010;116(22):4700-2. doi: 10.1182/blood-2010-06-289991 [published Online First: 2010/08/17]

328. Quach DH, Becerra-Dominguez L, Rouce RH, et al. A strategy to protect off-the-shelf cell therapy products using virus-specific T-cells engineered to eliminate alloreactive T- 
cells. J Trans/ Med 2019;17(1):240. doi: 10.1186/s12967-019-1988-y [published

Online First: 2019/07/26]

329. Rotolo A, Caputo VS, Holubova M, et al. Enhanced Anti-lymphoma Activity of CAR19iNKT Cells Underpinned by Dual CD19 and CD1d Targeting. Cancer Cell 2018;34(4):596-610.e11. doi: 10.1016/j.ccell.2018.08.017 [published Online First: 2018/10/10]

330. Poirot L, Philip B, Schiffer-Mannioui C, et al. Multiplex Genome-Edited T-cell Manufacturing Platform for "Off-the-Shelf" Adoptive T-cell Immunotherapies. Cancer Res 2015;75(18):3853-64. doi: 10.1158/0008-5472.Can-14-3321 [published Online First: 2015/07/18]

331. Yu S, Yi M, Qin S, et al. Next generation chimeric antigen receptor T cells: safety strategies to overcome toxicity. Mol Cancer 2019;18(1):125. doi: 10.1186/s12943019-1057-4 [published Online First: 2019/08/21]

332. Gargett T, Brown MP. The inducible caspase- 9 suicide gene system as a "safety switch" to limit on-target, off-tumor toxicities of chimeric antigen receptor T cells. Front Pharmacol 2014;5:235. doi: 10.3389/fphar.2014.00235 [published Online First: 2014/11/13]

333. Diaconu I, Ballard B, Zhang M, et al. Inducible Caspase-9 Selectively Modulates the Toxicities of CD19-Specific Chimeric Antigen Receptor-Modified T Cells. Mol Ther 2017;25(3):580-92. doi: 10.1016/j.ymthe.2017.01.011 [published Online First: 2017/02/12]

334. Griffioen M, van Egmond EH, Kester MG, et al. Retroviral transfer of human CD20 as a suicide gene for adoptive T-cell therapy. Haematologica 2009;94(9):1316-20. doi: 10.3324/haematol.2008.001677 [published Online First: 2009/09/08]

335. Tan AHJ, Vinanica N, Campana D. Chimeric antigen receptor-T cells with cytokine neutralizing capacity. Blood Adv 2020;4(7):1419-31. doi: 10.1182/bloodadvances.2019001287 [published Online First: 2020/04/10]

336. Tang XY, Sun Y, Zhang A, et al. Third-generation CD28/4-1BB chimeric antigen receptor $T$ cells for chemotherapy relapsed or refractory acute lymphoblastic leukaemia: a nonrandomised, open-label phase I trial protocol. BMJ Open 2016;6(12):e013904. doi: 10.1136/bmjopen-2016-013904

337. Avanzi MP, Yeku O, Li X, et al. Engineered Tumor-Targeted T Cells Mediate Enhanced Anti-Tumor Efficacy Both Directly and through Activation of the Endogenous Immune System. Cell Rep 2018;23(7):2130-41. doi: 10.1016/j.celrep.2018.04.051 [published Online First: 2018/05/17]

338. Zheng $W$, O'Hear CE, Alli R, et al. PI3K orchestration of the in vivo persistence of chimeric antigen receptor-modified T cells. Leukemia 2018;32(5):1157-67. doi: 10.1038/s41375-017-0008-6 [published Online First: 2018/02/27]

339. Rafiq S, Yeku OO, Jackson HJ, et al. Targeted delivery of a PD-1-blocking scFv by CAR-T cells enhances anti-tumor efficacy in vivo. Nat Biotechnol 2018;36(9):847-56. doi: 10.1038/nbt.4195 [published Online First: 2018/08/14]

340. Li AMH, G. E,; Dinofia, A. M et al. Checkpoint Inhibitors Augment CD19-Directed Chimeric Antigen Receptor (CAR) T Cell Therapy in Relapsed B-Cell Acute Lymphoblastic Leukemia. ASH 2018. San Diego: Blood, 2018:556. 


\section{Appendices}

Appendix 1: Quicksheet for CRS management algorithm adopted for the ENABLE Trial in Wellington Hospital

\begin{tabular}{|c|c|c|c|c|c|c|}
\hline \multicolumn{3}{|c|}{$\begin{array}{l}\text { Quick Sheet: Adult CRS Management } \\
\text { If your patient is experiencing any of the following after CAR T-cell therapy: } \\
\text { - Fever (temperature } \geq 38^{\circ} \mathrm{C} \text { ) } \\
\text { - Hypotension (systolic } \mathrm{BP} \leq 90 \mathrm{~mm} \mathrm{Hg}) \\
\text { - Hypoxia }\left(\mathrm{SpO}_{2} \leq 92 \%\right) \\
\rightarrow \text { Consider Cytokine Release Syndrome Management - see PML }\end{array}$} & \multicolumn{4}{|c|}{$\begin{array}{ll}\text { Key contacts } & \\
\text { Haematology SMO on-call: } & \text { Via CCDHB switchboard } \\
\text { PAR Team: } & \# X X X X \\
\text { CAR T- Trial 24hr contact: } & \text { XXXXXXX } \\
\text { CAR T - Principal Investigator: } & \text { (DC Robert Weinkove) XXXXXXX } \\
\text { CAR T - Clinical fellow: } & \text { (DC Philip George) XXXXXXX } \\
\text { CAR T - Research Nurse: } & \text { (Tess Ostapowicz) XXXXXX } \\
\end{array}$} \\
\hline Grade & Description & \multicolumn{2}{|l|}{ Management } & \multicolumn{3}{|c|}{ Tocilizumab administration - see Protocol } \\
\hline 1 & $\begin{array}{l}\text { Fever } \geq 38^{\circ} \mathrm{C} \\
\text { No hypotension } \\
\text { No hypoxia }\end{array}$ & \multicolumn{2}{|c|}{$\begin{array}{l}\text { Broad spectrum antibiotics if neutropenic, or if any suspicion } \\
\text { of sepsis (see Febrile Neutropenia policy) } \\
\text { Alert key contacts (must inform Haem SMO on call) } \\
\text { Monitor vital signs for hypotension, hypoxia, increase } \\
\text { frequency of observations while febrile and as clinically } \\
\text { indicated. } \\
\text { Daily blood tests } \\
\text { If persistent or refractory fevers lasting } \geq 3 \text { days } \\
\text { Consider Tocilizumab + corticosteroids (Consult with } \\
\text { Haematolog SMO) }\end{array}$} & \multicolumn{3}{|c|}{ 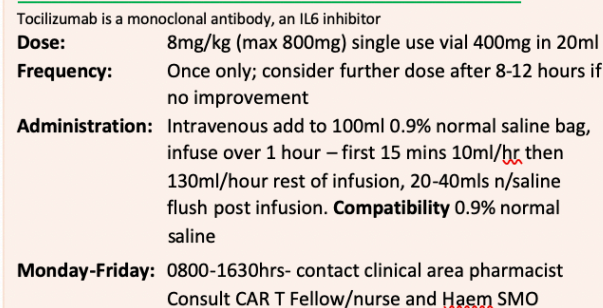 } \\
\hline 2 & $\begin{array}{l}\text { Hypotension (SBP }<90 \mathrm{~mm} \\
\mathrm{Hg} \text {; no vasopressors), } \\
\text { and/or } \\
\text { Hypoxia }\left(\mathrm{O}_{2} \text { via nasal }\right. \\
\text { prongs up to } 4 \mathrm{~L} / \mathrm{min} \text { ) }\end{array}$ & \multicolumn{2}{|c|}{$\begin{array}{l}\text { As for grade } 1 \text { CRS, plus } \\
\text { Initiate PAR team review for hypotension }+/ \text { - up to } 1.5 \mathrm{~L} \text { IV } \\
\text { fluids in up to } 2 \text { fluid boluses (avoid repeat fluid boluses) } \\
\text { Contact ICU to consider early vasopressor use } \\
\text { Administer } \mathrm{O}_{2} \text { via nasal prongs if hypoxic } \\
\text { Consider Tocilizumab + corticosteroids (Consult with } \\
\text { Haematology SMO) }\end{array}$} & \multicolumn{3}{|c|}{$\begin{array}{l}\text { After Hours: } \quad \begin{array}{l}\text { Contact } \mathrm{A} / \mathrm{H} \text { SMO and the on-call pharmacist if } \\
\text { Tocilizumab required via CCDHB switchboard }\end{array} \\
\begin{array}{l}1 \text { dose kept in fridge on Ward } 5 \text { North, } 2 \text { doses kept in } \\
\text { pharmacy. Maximum timeframe from call to administration }\end{array} \\
=2 \text { hours } \\
\text { - } \quad \text { Tocilizumab has a } \mathbf{2 4} \text { hour expiry once made } \\
\text { Corticosteroid dosing }\end{array}$} \\
\hline 3 & $\begin{array}{l}\text { Hypotension (SBP }<90 \mathrm{~mm} \\
\mathrm{Hg} ; 1 \text { vasopressor), and/or } \\
\text { Hypoxia needing high-flow } \\
\mathrm{O}_{2} \text { via nasal prongs } \\
\text { (>4L/min), facemask, non } \\
\text { rebreather or venturi } \\
\text { mask }\end{array}$ & \multicolumn{2}{|c|}{$\begin{array}{l}\text { As for grade } 2 \text { CRS, plus } \\
\text { MET call and ICU admission } \\
\text { Vasopressor and high-flow } \mathrm{O}_{2} \text { as needed } \\
\text { Consider Tocilizumab }+ \text { corticosteroids (Consult with } \\
\text { Haematology SMO) }\end{array}$} & \multirow{2}{*}{\multicolumn{2}{|c|}{$\begin{array}{l}\text { Dexamethasone: } \\
\text { Methylprednisolone: }\end{array}$}} & $\begin{array}{l}\text { 24 hour expiry once made } \\
\text { IV } 10 \mathrm{mg} 12 \text {-hourly, if no improvement } \\
\text { within } 8-24 \text { hrs, consider increasing to } 6 \\
\text { hrly } \\
\text { IV } 500 \mathrm{mg} \text { BD for } 3 \text { days, then }\end{array}$ \\
\hline 4 & $\begin{array}{l}\text { Hypotension }(\mathrm{SBP}<90 \mathrm{~mm} \\
\mathrm{Hg} ; 2+\text { vasopressors), } \\
\text { and/or positive pressure }\end{array}$ & \multirow{2}{*}{\multicolumn{2}{|c|}{$\begin{array}{l}\text { As for grade } 3 \text { CRS, plus } \\
\text { Consider high dose methylprednisolone (consult with } \\
\text { Haematology SMO on call) }\end{array}$}} & & & $\begin{array}{l}\text { IV } 125 \mathrm{mg} \text { BD for } 2 \text { days, then } \\
\text { IV } 60 \mathrm{mg} \text { BD for until CRS resolved }\end{array}$ \\
\hline & & & & \multicolumn{3}{|c|}{$\begin{array}{l}\text { Reference: Lee, D.W. et al., ASTCT Consensus Grading for Cytokine Release Syndrome } \\
\text { and Neurologic Toxicity Associated with Immune Effector Cells. Bijl Blood Marrow } \\
\text { Transplant, } 2019\end{array}$} \\
\hline
\end{tabular}




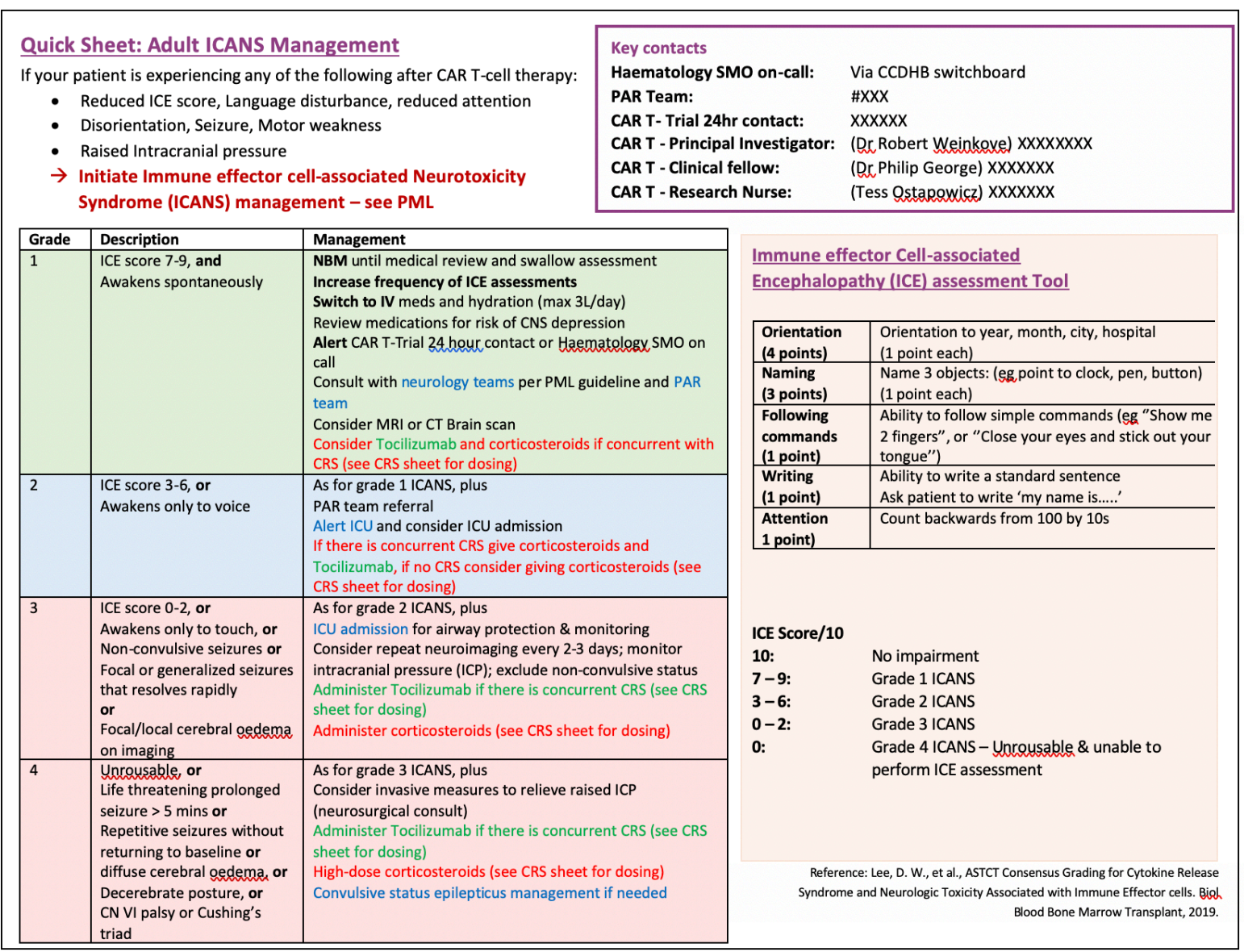

Appendix 3 Additional Manuscripts contributed to during Masters thesis

1) Selecting costimulatory domains for chimeric antigen receptors: functional and clinical considerations.

Weinkove, R.; George, P.; Dasyam, N.; McLellan, A, D.; Journal of Translational and Clinical Immunology, 2019 May 11;8(5):e1049

Extracts of this published manuscript are provided in Section 2.6 Selecting costimulatory domains for chimeric antigen receptors: functional and clinical considerations. 


\begin{abstract}
:
Costimulatory signals are required to achieve robust chimeric antigen receptor (CAR) T-cell expansion, function, persistence and antitumor activity. These can be provided by incorporating intracellular signalling domains from one or more T-cell costimulatory molecules, such as CD28 or $4-1 \mathrm{BB}$, into the CAR. The selection and positioning of costimulatory domains within a CAR construct influence CAR T-cell function and fate, and clinical experience of autologous anti-CD19 CAR T-cell therapies suggests that costimulatory domains have differential impacts on CAR Tcell kinetics, cytotoxic function and potentially safety profile. The clinical impacts of combining costimulatory domains and of alternative costimulatory domains are not yet clearly established, and may be construct- and disease-specific. The aim of this review is to summarise the function and effect of established and emerging costimulatory domains and their combinations within CAR T cells.
\end{abstract}

2) B-cell Prolymphocytic Leukaemia With a t(4;14) FGFR3/IGH Translocation: Response to Ibrutinib.

George, P.; Brown, A.; Weinkove R. Pathology, 2020 Apr 26;S0031-3025(20)307911, doi: 10.1016/j.pathol.2020.03.005

\footnotetext{
Abstract:

Translocations between the transcriptionally-active IgH locus at chromosome 14q32 and proto-oncogenes, leading to oncogene dysregulation, are well-characterised events in many B- cellmalignancies.1 For example, the $t(4 ; 14)(p 16.3 ; q 32.2)$ translocation leads to dysregulated expression of two genes: fibroblast growth factor receptor 3 (FGFR3) and myeloma SET domain protein (MMSET). Approximately $10 \mathrm{e} 15 \%$ of patients with plasma cellmyeloma harbour a $\mathrm{t}(4 ; 14)(\mathrm{p} 16.3 ; \mathrm{q} 32.2)$ translocation, which is associated with a poor prognosis. However, translocations involving 14q32 are unusual in chronic lymphocytic leukaemia (CLL), the most prevalent B-cell malignancy in Western countries. We report clinical and morphological features of a case of CLL with a rarely-reported $t(4 ; 14)(\mathrm{p} 16.3 ; \mathrm{q} 32.2)$
} 
IGH:FGFR3 translocation and early transformation to B-cell prolymphocytic leukaemia (B-PLL), and document clinical response to ibrutinib, an orally bioavailable Bruton tyrosine kinase (Btk) inhibitor.

3) CAR T-cells arrive in Australasia Part 2: Manufacturing, regulation, and ethical considerations.

George P, Ruka M, Perry M, Dickinson M, Weinkove R.

Manuscript in advanced preparation for submission to the Internal Medical Journal.

\begin{abstract}
:
Chimeric antigen receptor (CAR) T-cells are an emerging standard of care for certain relapsed and refractory B-cell cancers. As a personalised cell and gene therapy with specific and potentially life-threatening toxicities, CAR T-cells present unique manufacturing, logistical, regulatory and ethical challenges. This review summarises the manufacture of CAR T-cells from the perspective of a treating site, and discusses logistical issues, accreditation of treatment sites and the role of registries for longterm follow-up. Key regulatory bodies, legislation and guidance applicable to CAR Tcell therapies in Australia and New Zealand are outlined. Consideration is given to ethical issues, including equity of access, off-label and compassionate use, and the applicability of CAR T-cell therapies to specific groups, including Jehovah's Witnesses, pregnant or breast-feeding women and HIV positive individuals.
\end{abstract}

